䍠
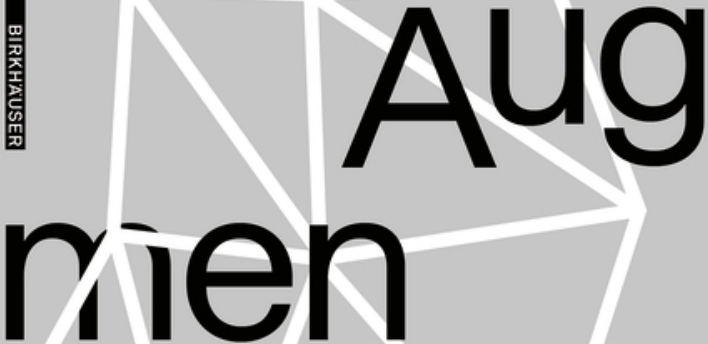

Das Design von kartenbasierten Interfaces
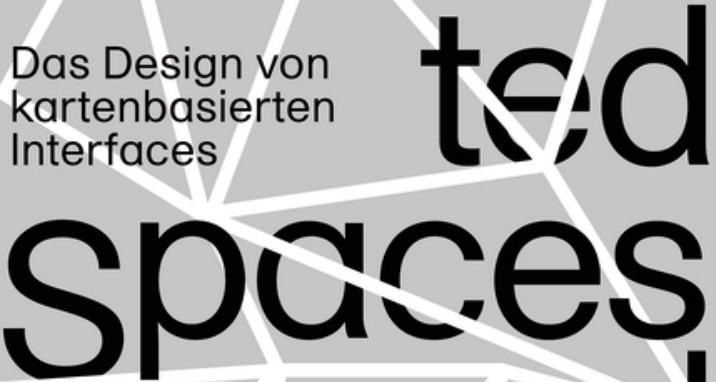

Christine Schranz
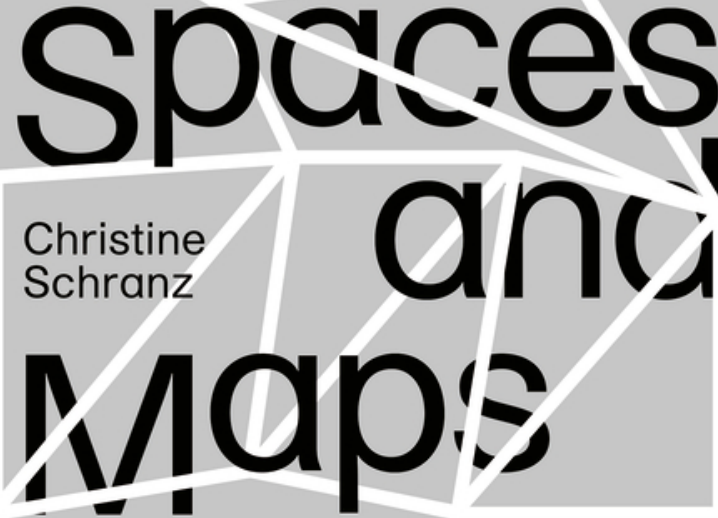


\section{Augmented Spaces and Maps}

Das Design von kartenbasierten Interfaces

Christine Schranz

Birkhäuser Basel 
«Wir formen unsere Werkzeuge,

und dann formen unsere Werkzeuge uns.»

Marshall McLuhan 


\section{Kann Google einen Krieg auslösen?}

Karten sind faszinierende Bildwelten, die einen in eine Fantasiewelt zu katapultieren vermögen. Karten sind aber auch politisch, sie blenden ein, lassen etwas weg oder verzerren das Abbild der Welt. Wie für viele Designer_innen und Künstler_innen, übten Karten auf mich seit jeher eine Faszination aus. In meinem Kinderzimmer verfolgte ich auf einer Weltkarte mit dem Finger die Geschäftsreisen meines Vaters. Als Jugendliche begann ich selbst zu reisen und markierte auf derselben Karte die von mir besuchten Länder und Orte. Später machte ich die Erfahrung, dass Karten keinen Anspruch auf Objektivität haben und einer Generalisierung unterliegen. Auf einer Fahrradtour mit meinem damaligen Freund durch Kuba hatten wir eine Route herausgesucht, die, wie wir erst später feststellten, durch eine militärische Sperrzone führte. Auf unserer Karte war das nicht passierbare militärische Gebiet in der Guantánamo-Bucht - auf welchem sich später das Gefangenenlager der US-Navy befand - nicht verzeichnet und der auf der Karte eingezeichnete Weg existierte nicht. Noch heute besitze ich die Karten, die mich durch meine Kindheit und Jugendzeit begleitet haben. Neben meinem persönlichen Wissensschatz sind sie vor allem Zeitdokumente und geben Auskunft über politische und historische Veränderungen. Viele Grenzen auf diesen Karten existieren - mit dem Ende des Kalten Kriegs und der territorialen Zersplitterung im Nahen Osten - heute nicht

Ingo Günther: «Mauern, Zäune, Barrieren» (2016) aus dem Projekt Worldprocessor. Die Linien kennzeichnen die weltweit existierenden sowie sich in Planung befindlichen Grenzmauern.

Die entsprechenden Länder sind mittels eines Codes identifizierbar. 
mehr, neue sind dafür dazugekommen. Diese Karten, wie Karten im Generellen, sind epistemologische Speicher. Meine eigenen Erfahrungen und Erlebnisse haben sich darin abgelagert, geopolitische Veränderungen und Wissen lassen sich herauslesen.

Wie die Kartografen John Krygier und Denis Wood betonen, sollten Nutzer und Produzent_innen Karten immer kritisch gegenüberstehen: «[M]maps have the power and potential for failing that every humancreated object has.» (Krygier\&Wood 2005: 7) Dass das Kartenmaterial von Drittanbietern meist ohne vorgängige genaue Prüfung übernommen wird, musste auch die Schweizer Fluggesellschaft Swiss erfahren. Im Oktober 2018 löste eine veraltete Karte auf einem Swiss-Flug nach Israel für virale Aufmerksamkeit. Das Personal vergass, die Kartenanzeige auszuschalten, woraufhin auf dem Display ein Ort zu sehen war, der nicht mehr existiert. Zu sehen war das Dorf Asch-Schaich Muwannis, das bereits während des Palästinakriegs 1948 durch das israelische Militär eingenommen und weitgehend zerstört wurde. Für die arabischen Bewohner_innen gilt der Ort seitdem als Symbol für die Vertreibung ihrer Kultur.

Waren für Kartendienste bisher staatliche Institutionen wie SwissTOPO (Schweiz), Geoportal (Deutschland) oder Geoland (Österreich) zuständig, werden diese in digitalen Kulturen zunehmend von privaten Internetunternehmen wie etwa Alphabet (Google Maps) oder Microsoft (Bing) verdrängt. Die weltweit am häufigsten verwendete Karte ist Google Maps. Diese Verschiebung in der geografischen Praxis zieht weitreichende Veränderungen und Konsequenzen nach sich. Diese betreffen einerseits die Urheberschaft von Karten im weitesten Sinne (Internetunternehmen sowie Informatiker_innen, Prosumer_innen und langfristig und immer stärker auch Algorithmen oder Maschinen), anderseits die Kartengestaltung und der Karteninhalt auch im Zusammenhang mit den dahinterstehenden Technologien (vgl. Felgenhauer \& Gäbler 2018; Ash/Kitchin/Leszczynski 2019 u. a.). Das Abtreten von geografischer Verantwortung an private Unternehmen kann handfeste politisch-militärische Folgen haben, wie folgendes Beispiel verdeutlicht. Vor einiger Zeit kam es beinahe zu einem bewaffneten Konflikt zwischen Nicaragua und Costa Rica, welcher nur durch eine Entschuldigung von Google abgewendet werden konnte. Was war geschehen? Soldaten der nicaraguanischen Armee besetzten ein Naturschutzgebiet auf einer zu Costa Rica gehörenden Insel, die aber auf Google Maps als zu Nicaragua zugehörig einge-

1 www.sueddeutsche.de/politik/2.220/costa-rica-grenzkonflikt-wegen-googlemaps-biegen-sie-jetzt-ab-ueber-die-grenze-1.1021817 (abgerufen am 20.11.2018). 
zeichnet war. Es ist zwar nicht das erste Mal, dass Google im Zusammenhang mit einer Grenzstreitigkeit genannt wird. Bemerkenswert am obigen Beispiel ist aber, dass der Internetkonzern als Kartenurheber sich öffentlich für seinen Fehler entschuldigt und die Verantwortung dafür übernommen hat.

Die Popularität der Karte als Diskursobjekt ist jedoch nicht nur dem Internet beziehungsweise der Digitalisierung geschuldet, sondern auch einem Umbruch in der Geografie als Disziplin. Mit der kritischen Geografie (vor allem im englischsprachigen Raum und unter dem Begriff Humangeografie [Human Geography] bekannt geworden, u. a. Harvey 1990) beziehungsweise einer kritischen Kartografie (Critical Cartography, insbesondere seit/mit Harley 1989; Harley 2001 u. a.) oder auch Radical Cartography formiert sich seit den späten 1980er-Jahren eine Gegenbewegung und Kritik an den vorherrschenden kartografischen Praxen (vgl.z. B. Wood 1992; Crampton 2010; Dodge 2016 u. a.). Die Karte als Ausdruck von Hegemonie, Kontrolle und Macht (zu Power siehe auch Brotton 2014; Marshall 2017) und ein sich an Eliten orientierendes Kartenbild, das die allgemeingültigen Regeln und Normen definiert, wird zunehmend hinterfragt. Die Kritik gilt unter anderem der Marginalisierung beziehungsweise der gängigen Mercator-Projektion, die auf einem eurozentrierten Weltbild aufbaut. Mit der aktuellen geopolitischen Weltlage, dem Bedeutungsverlust von Europa und dem drohenden Zerfall der EU, den Konflikten im Nahen Osten, den Migrationsströmen, der Dekolonialisierung und den Bottom-up-Bewegungen (bis hin zum Aufdecken geheimer Praxen wie zum Beispiel der weltweiten CIA-Gefängnisse) hat die Karte als visuelles Episteme (Knowledge Production) enorm an Bedeutung gewonnen. Insbesondere im Kontext des Post-Kolonialismus sind alternative Kartierungsstrategien beliebt geworden. Solche Karten sind nicht zur Orientierung gedacht, sondern werden vielmehr dafür verwendet, um zum Beispiel auf während der Eroberungs- und KoIonialzeit zu Unrecht annektierte Gebiete aufmerksam zu machen (vgl. Wood/Fels/Krygier 2005; Kollektiv Orangotango+ 2018 u. a.). Im Zusammenhang mit dem Internet und der Digitalisierung beziehungsweise der dadurch entstandenen Möglichkeit der Kartenherstellung durch Nicht-Kartograf_innen, spitzt sich der Diskurs nochmals zu. Seit der Einführung des mobilen Internets ist die Karte als visuelles Episteme ununterbrochen populär, was die zahlreichen Projekte, Publikationen und Visualisierungen belegen. Seit Web 2.0 (Inhalte werden nicht nur konsumiert, sondern auch produziert) können mit Karten nutzergenerierte Inhalte erstellt werden. Dadurch ist ein geospatiales Web entstanden - das Geo-Web. Verortungstechnologien 
sowie GIS (Geoinformationssysteme ${ }^{2}$ ) haben die Demokratisierung der Kartenherstellung vorangetrieben und mit API (Application Programming Interface) wurden Anwendungsschnittstellen zum Austausch und zur Einbindung digitaler Karten geschaffen. Mit dem GeoWeb werden digitale Karten immer bedeutsamer und die kostenlosen Online-Mapping-Services Google Maps und Google Earth dominieren den Markt. Selbst auf dem iPhone von Apple wurden anfänglich die Karten von Google verwendet, da das Unternehmen über kein eigenes Kartenmaterial verfügte.

Durch nutzergenerierte Karten entwickelt sich ein räumlicher Dialog und mit raumbasierten Technologien wie Augmented-Reality-Applikationen (App) entsteht eine Nähe zu materiellen Kulturen (siehe auch Hahn 2014; Samida 2014 u. a.) und dem physischen Raum. Bei Karten als epistemische Objekte geht es nicht nur um die Visualisierung von geografischem Wissen beziehungsweise um die Darstellung der geografischen Realität in kleinerem Massstab, sondern um eine methodologische, theoretische oder auch persönliche Annährung an eine gesellschaftliche Konstellation, einen Zustand oder Ähnliches. Mittels der Karte beziehungsweise mit Kartierungsstrategien werden Quellen und nicht autorisierte Veröffentlichungen gesammelt, offengelegt und kritisch hinterfragt (Holmes 2007; Thompson 2008; Kurgan 2013; Franke/Weizman/Sheikh 2014; Kollektiv Orangotango+ 2018 u. a.). Solche, vor allem bei Künstler_innen und Designer_innen beliebte Formen eines radikalen Mappings, finden sich zum Beispiel bereits in den Karten des deutschen Künstlers Hans Haacke, etwa in der Arbeit «Shapolsky et al. Manhattan Real-Estate Holdings, A RealTime Social System, as of May 1 » (1971) oder in dem Werk «BCl, ICIC\&FAB» (1972 - 1991) des amerikanischen Künstlers Mark Lombardi (1951-2000). Beide Künstler haben ohne Hilfe des Internets und in akribischer Handarbeit brisante Kartografien erstellt, die auf Verflechtungen und Skandale in Wirtschaft und Politik hinweisen. (Abb.2)

Im Zeitalter von Internet und Digitalisierung sind solche radikalen Tendenzen als Counter-Maps beziehungsweise als kritische, alternative oder auch subversive Kartografie bekannt geworden. Sie sind Ausdruck von Kritik an den herrschenden Systemen und Regeln und werden unter anderem zur Aufdeckung beziehungsweise zur bildlichen Visualisierung von vertuschten Affären oder Skandalen eingesetzt. Darüber hinaus sind sie eine Zuwendung zu subjektiv - und von der jeweiligen Kultur abhängig - erlebtem Raum und hinterfragen die Objektivität, also die Realität und Repräsentation von Karten.

2 Die wichtigsten technischen Begriffe werden in der Einleitung kurz erörtert und dann in den jeweiligen Kapiteln näher besprochen. 
Die Karte als Protest, als visuelles Episteme hat seitdem viele Künstler_innen und Designer_innen inspiriert und zur Gestaltung von Karten als Gegenentwürfe zu gängigen Weltbildern, Hierarchien und Organisationen angeregt beziehungsweise ein weltweites kritisches, kartografisches Denken begünstigt. Zeitgenössische Designerkollektive oder Grafiker_innen, die sich in ihrer Arbeit mit Counter-Maps engagieren, sind beispielsweise das Bureau d'Études (Léonore Bonaccini and Xavier Fourt),

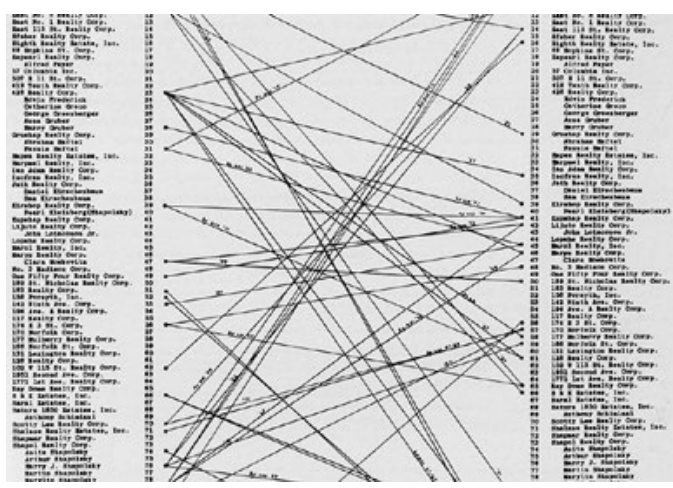
Hans Haacke: «Shapolsky et al. Manhattan Real-Estate Holdings, A Real-Time Social System, as of May 1, 1971» (1971) (Detail) der Grafiker Philippe Rekacewicz (Atlas der Globalisierung, 2009) oder auch Organisationen wie Counter Cartographies Collective ${ }^{3}$. Das Bureau d'Études ist für seine Kartografien politischer, sozialer und wirtschaftlicher Systeme bekannt geworden. In der Publikation An Atlas of Agendas. Mapping the Power, Mapping the Commons (zusammen mit Brian Holmes und Freek Lomme, 2013) versammelt die Gruppe hervorragende Visualisierungen, die eine andere Sichtweise auf die Dinge ermöglichen. Für die Gestaltung nutzten die Designer_innen digitale Daten, Informationen und Netzwerke aus dem Internet. Die 2003 erstellte Karte «Governing by Networks» etwa verdeutlicht die wechselseitigen Verflechtungen und Abhängigkeiten innerhalb globaler Medienkonglomerate. (Abb. 3) Die Karte ist Teil einer visuellen Analyse zum transnationalen Kapitalismus. Sie enthüllt, was meist unsichtbar bleibt, und stellt zumeist getrennte Sachverhalte in einen grösseren Zusammenhang.

Zürich, Mai 2019 


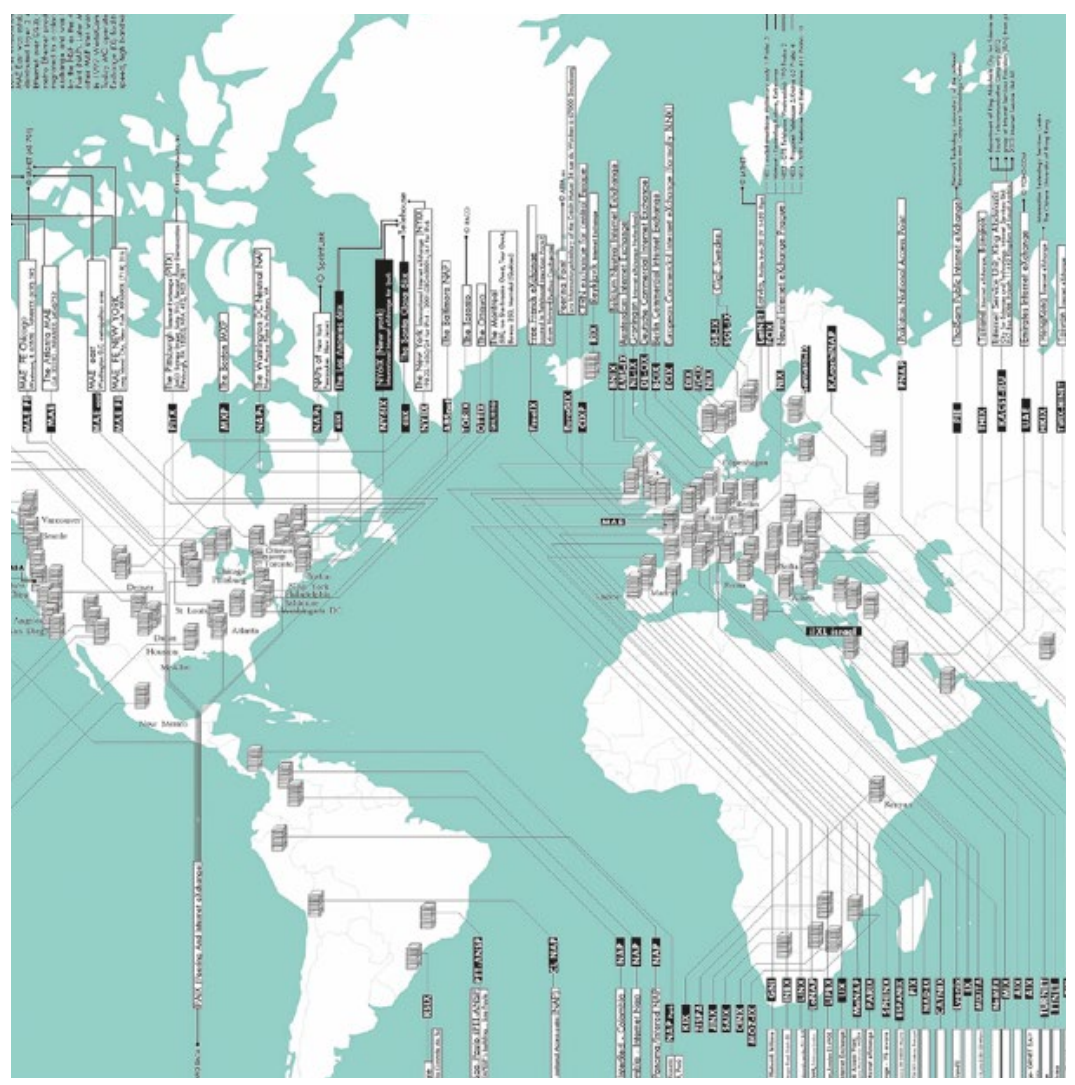

Léonore Bonaccini und Xavier Fourt vom Bureau d'Études: «Infocapital Exchangers» (2003) (Detail) 

Inhaltsverzeichnis

Einleitung Konvergenz von Raum, Medien und Design

Theorie

1 Raum - Der digitale Spatial Turn

1 Der Raumwandel in digitalen Gesellschaften 27

$\begin{array}{lll}1 & \text { Der Spatial Turn } & 30 \\ 2 & \text { Raumproduktion im Geo-Web } & 34 \\ 3 & \text { Räumliche Analysen und } & 38 \\ & \begin{array}{l}\text { Datenvisualisierungen } \\ 4\end{array} & \text { Die Raumerweiterung } \\ 5 & \text { Abhängigkeit von } & 43 \\ & \text { Raum und Daten } & 45\end{array}$

2 Augmented Space - Human Environment Interfaces 47

1 Augmented Reality 51

2 Über Augmented Reality 56

$3 \quad$ Raum-Mensch-Interaktion $\quad 59$

2 Interface - Die Ästhetik von medialen Oberflächen 65

1 Die Gestalt der Karte $\quad 67$

1 Analoge, digitale und $\quad \mathbf{7 0}$

2 Die Kartengestaltung $\quad 74$

$3 \quad$ Bezug zur Perspektive $\quad 79$

$4 \quad$ Von Human-centered Design 85 zum Design für Maschinen

2 Die Benutzerschnittstelle zwischen Mensch-Computer $\quad 89$ (oder System)

$1 \quad$ Die grafische Benutzeroberfläche 92

$2 \quad$ Natural User Interface - 93 akustische, haptische und muskuläre Signale

$3 \quad$ Interface-Gestaltung und 95

$4 \quad$ Interface-Kulturen und das 96 Interaktionsparadigma

3 Macht - Die neuen Kartograf_innen $\quad 99$

1 Karten als Abbild verschiedener Weltentwürfe 101

1 Die Frage nach dem Zentrum: 104

2 Google - wie ein Internetunter- 107 nehmen zur KartografieGrossmacht wurde (Top-down)

3 OpenStreetMap - ein alternatives Kartierungs-Common (Bottom-up)

$4 \quad$ Kritische Kartografie und $\quad 115$

5 Counter, Critical, Radical 119 
Inhaltsverzeichnis

2 Daten, Informationen und Wissen

1 Georeferenzierte Inhalte

2 Verwandlung von Daten

in Information und Information in Form

3 Die Karte als Informationsgrundlage

4 Wissen, Epistemologien und Information im Raum

$4 \quad$ Navigation - Die Karte kommt in Bewegung

1 Vom Kartenschauen zum Navigieren

1 Räumliches Wissen - externe Referenzsysteme und innere Karten

$2 \quad$ Analoge und digitale

3 Weitere Navigationsformen Routenwissen und Wegstrecken

$4 \quad$ Limitationen und Potenziale digitaler Navigationssysteme

2 Die Praxis des Erzählens

$1 \quad$ Kartierungen des eigenen $\quad 164$

$2 \quad$ Locative Media 168

3 Erzählung im (vernetzten) Raum 172

Nachwort Design im Wandel

Anwendung

5 Augmented Space - Am Beispiel des Zollfreilagers auf dem Dreispitz

1 Das Basler Zollfreilager und die Praxis der Kunstfreilager

1 Das Basler Zollfreilager 190

2 Zollfreilager und Freihandels- 193

3 Der Kunstmarkt

194

$4 \quad$ Nationale und internationale Zollfreilager mit Kunstbezug

2 Die Applikation Augmented Space

1 Die historische Ebene

2 Die spekulative Perspektive

3 Fazit

Anhang

Kurzbiografie

Literaturverzeichnis

Abbildungsverzeichnis

Projektbezogene Forschungsresultate aus Lehre und Forschung 



\section{Konvergenz von Raum, Medien und Design}

Mit der Konvergenz von Raum, Medien und Design verändert sich nicht nur die Praxis, sondern auch die Medien beziehungsweise die Oberflächen, die es zu gestalten gilt, verändern sich. Der Design- und Kognitionswissenschaftler Donald A. Norman beschreibt den momentanen Zustand anlässlich der Verleihung seiner Ehrendoktorwürde wie folgt: «Getrieben von tiefgreifendem Fortschritt in der Informatik und den Computertechnologien, in den Bereichen der Sensorik, der Kommunikation und der Displays, ist die Welt der Technik einem raschen Wandel unterworfen, der zunehmenden Einfluss auf Gesellschaft und Umwelt nimmt. (Norman, zit. n. Denzinger 2018: 17) Wie nie zuvor steht Design heute vor der Aufgabe, Ort und Lebenswelt in ihrer medialisierten Form neu zu denken und zu gestalten. Dieser Shift im Design beziehungsweise innerhalb digitaler Kulturen bringt neue Berufsfelder für Designer_innen oder gänzlich neue Berufe wie Creative Coder_innen und Content-Manager_innen hervor, welche hybride Räume ${ }^{4}$ und Orte entwerfen und gestalten. Eine neue Generation von Gestalter_innen ist nun von jungen Kund_innen aufgefordert, Räume zu gestalten, die ästhetisch und zugleich funktional sind (vgl.Colomina \&Wigley 2016: 260). Der Raum $^{5}$, insbesondere in Verbindung mit neuen Kommunikationstechnologien, wurde im Kommunikationsdesign und in der Designforschung bisher nur marginal behandelt. Dies ist der bis zur Digitalisierung (mobiles Internet, digitale Netzwerke und Infrastrukturen) mehrheitlich analogen, zweidimensionalen Oberflächen geschuldet. Das Designer-Kollektiv Metahaven (Vinca Kruk und Daniel van der Velden) beschreibt den Wandel von Papier zur Oberfläche in seinem Design-Manifest White Night. Before A Manifesto (2008). Dieses behandelt die nach wie vor aktuellen Bedingungen einer zeitgenössischen Designpraxis und die daraus resultierenden politischen Konsequenzen. Im aktuellen Jahrhundert der Digitalisierung sowie im Kontext des Zeitalters des Entwerfens (in welchem die Welt mehrheitlich durch den Menschen gestaltet und beherrscht ist und unter dem Begriff des Anthropozäns diskutiert) wird sich Design, das heisst die Designwissenschaft zunehmend mit der Gestaltung von digitalen

4 Der Begriff «hybride Räume` wird in Kapitel 1.2 näher erörtert.

5 Der Begriff «Raum〉 ist ein mehrdeutiger und konzeptionell unscharfer Begriff und steht hier synonym für etwa Ort, Lebenswelt, Infrastruktur. Er fokussiert im Folgenden auf das Phänomenal-Räumliche und gleichzeitig auf das KonkretPhysische. Näher erörtert wird der Raumbegriff in Kapitel 1. 


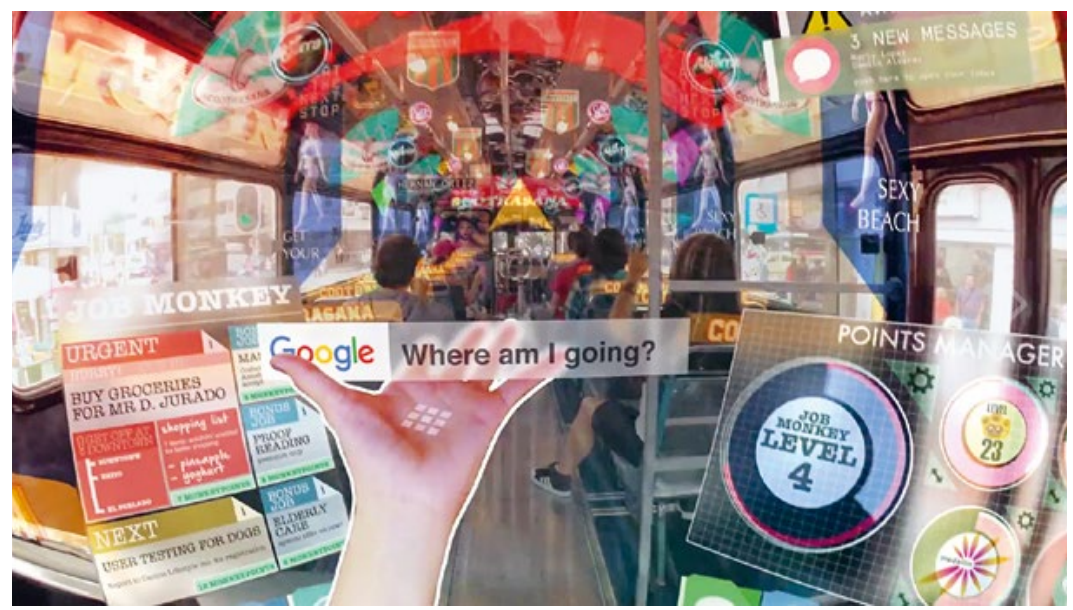

Oberflächen, Interfaces ${ }^{6}$ und Räumen (oder auch Systemen) auseinandersetzen müssen. Dabei werden Grundlagen zum und im Umgang mit Raum bezieKeiichi Matsuda: «Hyper-Reality» zeigt anhand eines utopischen Films, wie sich künftig das Leben (hier konkret bei einer Busfahrt) beziehungsweise die virtuellen und realen Erlebnisse durchdringen und miteinander verschmelzen. hungsweise zu räumlichen Informationen zentral. (Abb.4)

Weshalb dieses Buch? Diese Publikation wurde aus einer designzentrierten Perspektive verfasst, bringt jedoch Wissen aus Disziplinen wie etwa Raumtheorie, Kartografie, Medienwissenschaft, Human Computer Interaction $(\mathrm{HCl})$ mit ein. Bisherige Publikationen fokussieren nur auf einen kleinen Ausschnitt oder bestimmte technologische Aspekte, oftmals wird Design - auch als ästhetische Disziplin - marginal behandelt. Deshalb gilt es nun, das Thema auch aus einem holistischen - interdisziplinären - Blick anzugehen. Diese Publikation erhebt dabei jedoch nicht den Anspruch, historisch lückenlos oder chronologisch aufgearbeitet zu sein, sondern behandelt Prinzipien, Konzepte und Beispiele für und aus dem Design und der Kunst. Sie richtet sich insbeson-

6 Als Interface sind hier Schnittstellen im Zusammenhang mit Design und einer Computeranwendung gemeint. 
dere an Designer_innen, Coder_innen, Szenograf_innen etc., also jene Zielgruppen, die mit der Gestaltung der oben beschriebenen Thematik beschäftigt sind oder sein werden. In den ersten vier zum Theorieteil zählenden Kapiteln wird in die Grundlagen eingeführt, die integraler Bestandteil eines solchen Designprozesses sein sollten. Im fünften Kapitel, dem Praxisteil, wird ein Beispiel aus meiner Designpraxis vorgestellt, das parallel zum Verfassen dieses Textes entstanden ist. Die zentrale These dabei war: Mit dem Erstarken des Raums (einer Hinwendung zu räumlichen Themen insbesondere mit der Verortungstechnologie GPS [Global Positioning System] und seit dem Geo-Web) werden neue Zugänge zu Daten, Informationen und Inhalten (neuartige Navigations- und Erzählformen, die sich im Raum manifestieren sowie neue Formen von Ästhetiken, welche sich physisch und digital materialisieren und durchdringen) bedeutsam. Die Karte als räumliche Repräsentation wird dabei zu einem zentralen Interface. Neben Verortungstechnologien sind räumliche Technologien wie Augmented Reality (AR) oder das Internet der Dinge (engl. Internet of Things, loT) sowie die Prinzipien von Ubiquitous Computing und Mixed Reality paradigmatisch für einen solch situierten Designbegriff. Der hier verfolgte Ansatz ist inter- beziehungsweise multidisziplinär. Scheint dieser im Design als eine Selbstverständlichkeit, wird er in eher disziplinär ausgerichtet Disziplinen wie den Geisteswissenschaften jedoch teils immer noch mit Argwohn beobachtet. Entsprechend diesem Ansatz geht es nachfolgend nicht um die lückenlose Aufbereitung der hier angeschnittenen Theorien, sondern darum, Konzepte und Methoden für das Design und die Designpraxis verfügbar zu machen. Zur Verdeutlichung der Durchdringung von Theorie und Praxis werden wo immer möglich und hilfreich - designrelevante Beispiele herangezogen, die die Disziplin voranbringen oder -gebracht haben.

Im Zusammenhang mit der Georeferenzierung von digitalen Daten und Informationen (im Folgenden georeferenziert) sowie mit mobilen und ubiquitären Geräten drängen sich zwei einflussreiche Diskussionen auf: einerseits die Erweiterung des Begriffs Spatial Turn zu einem digitalen Raumwandel, andererseits eine Neuperspektivierung der Karte als Schnittstelle zwischen Mensch-Computer (oder System) und Raum. Anhand der Karte beziehungsweise der Karte als Interface sollen diese Neuperspektivierungen für Design und die Designwissenschaft aufgezeigt werden. War die Gestaltung der Oberfläche lange auf das Papier beschränkt, eröffnen sich mit Geoinformationssystemen (GIS - räumliche Informationssysteme zur Erfassung von Daten) neue Formen und Dimensionen für das Design, spezifisch, was Ästhetik, Navigation und Storytelling betrifft. Daraus ergeben sich einflussreiche Diskussionen in der Designdebatte und 
markante Umbrüche, die unter anderem folgende sieben Themenfelder adressieren:

1. Georeferenzierte Inhalte haben nicht nur den Umgang mit Raum verändert, sie haben auch die Karte paradigmatisch zur Schnittstelle zwischen Mensch und Computer (oder System) und Raum erhoben. Mit der Lancierung von Google Maps 2004 (Entwickler Lars und Jens Rasmussen) wurde es möglich, eine Datenbank an ein kartografisches Interface (oder System) zu knüpfen: Google wusste fortan nicht nur, wer wir sind, sondern auch, wo wir uns befinden. Oder wie es der ehemalige Produktmanager Lior Ron von Google Maps treffend formuliert hat: "[V]on Google Maps zu Google on maps.» (Zit. n. Döring \& Thielmann 2009b: 34) Wegweisend für den Paradigmenwechsel waren Navigationsgeräte für Fahrzeuge, die mit GPS, einem globalen Satellitensystem zur Positionsbestimmung, ausgestattet waren. Seither werden GPS-Signale ubiquitär eingesetzt: sei es zur Lokalisierung eines Standorts, der Ortung von Wildtieren, dem Lenken von Raketen oder für die Navigation zu einem Urlaubsziel.

2. Einen Umbruch im räumlich-kartografischen Denken beziehungsweise in der Darstellung der Welt markierte die Erfindung von Earth Viewer durch das Unternehmen Keyhole (2001, Gründer Michael T.Jones). Dieses wurde 2004 von Google aufgekauft und die Software in Google Earth umbenannt. Für Google Earth wurden erstmals Satellitenbilder mit Kartenmaterial kombiniert und zugleich mit GIS-Daten (verschiedene Datenformate zur Verarbeitung von geografischen Informationen) verknüpft. Das Konzept des stufenlosen Zoomens bei Google Earth basiert auf dem zentralen Gestaltungselement im Kurzfilm Zehn Hoch (engl. Originaltitel: The Powers of Ten; USA 1977, R.: Charles Eames \& Ray Eames). Satellitenbilder haben die Kartografie nicht nur durch einen einfachen Zugang revolutioniert, sondern zugleich zugänglicher (und in diesem Sinne auch demokratischer ${ }^{7}$ ) gemacht. Heutzutage werden Satellitenbilder insbesondere dort als Quelle zur Aufklärung herangezogen, wo aufgrund geopolitischer Krisen oder Kriege eine Begehung vor Ort unmöglich geworden ist, wie beispielsweise in Syrien. Bekanntheit erlangten Satellitenbilder früher unter anderem im Golfkrieg, bei welchem weltweit der Einmarsch amerikanischer Truppen über Satellitendaten verfolgt werden konnte. Diese Form der Datensammlung und -auswertung ist insbesondere mit der Forschergruppe Forensic Architecture bekannt geworden, aber auch in einem Kunstkontext, zum Beispiel durch 
«Zone*Interdite» (seit 2000) von Christoph Wachter und Mathias Jud $^{8}$ oder «Terminal Air» ${ }^{9}$ (2007) von Trevor Paglen.

3. Mit der exponentiellen Zunahme an georeferenzierten Inhalten (seit Web 2.0) stellen Karten nicht nur Schnittstellen dar, sondern ermöglichen auch neuartige Wissenszugänge und Erkenntnisproduktionen (visuelle Epistemologien). Die Karte wird hierfür zur Grundlage und zeichnet sich dadurch aus, dass sie zur Visualisierung, Verhandlung und Kritik drängender gesellschaftlicher Fragen herangezogen wird (Mapping als gestalterisch-künstlerische Tätigkeit und Forschungsabsicht). Entsprechend nimmt Mapping in der Arbeit von Designer_innen und Künstler_innen eine zentrale Rolle ein. Dies beinhaltet einerseits die Visualisierung von Daten (Infografiken, Diagramme), andererseits auch die vermehrte Schaffung eines alternativen Zugangs zu Daten und Information, wie beispielsweise die Arbeit der deutschen Designerin Gerlinde Schuller zu Wissensepistemologien ${ }^{10}$ oder die Atlanten des niederländischen Designers Jost Grootens ${ }^{11}$. Weitere Ansätze sind etwa die gestalterischen Mittel und Strategien der Designerkollektive Metahaven ${ }^{12}$ oder Bureau d'Études ${ }^{13}$ zu Ideologiekritik und politischem Design. Karten sind Ausdruck visueller Kulturen und ermöglichen einen methodologischen und erkenntnistheoretischen Zugang zu Raum. Darüber hinaus bieten sie die Möglichkeit, Daten und Informationen in räumliche Zusammenhänge zu bringen und Bezüge herzustellen.

4. Daten, hier im Speziellen georeferenzierte Daten - also solche, die eine räumliche oder örtliche Komponente haben -, gelten als der neue Rohstoff, als das Kapital des 21. Jahrhunderts. Das Internet hat wie in vielen anderen Bereichen auch - bezüglich der Kartografie eine Art von «totaler Demokraties geschaffen. Mittels Mashup (Kombination von Text, Bild und Audio mit Kartendaten) wurde es theoretisch jedermann möglich, eigene Karten zu gestalten, zu teilen oder zu verbreiten. Durch die Veränderungen in der Produktion von Kartenmaterial und in der Urheberschaft von Geodaten entstehen jedoch auch (neue) Ungleichheiten und Hegemonien: Mit Google Maps der weltweit am häufigsten verwendeten Karte - ist, wie weiter oben bereits illustriert wurde, erstmals ein Internetunternehmen für das

8 Die beiden Künstler sammeln im Internet Daten (Bruchstücke) zu militärischen Speerzonen und stellen die Informationen auf ihrer Plattform neu zusammen, siehe auch www.wachter-jud.net/Zone-Interdite.html.

9 www.paglen.com/_oldsite/pages/projects.htm.

10 www.theworldasflatland.net/cv.htm.

11 www.joostgrootens.nl.

12 www.metahaven.net.

13 www.bureaudetudes.org. 
medialisierte Bild beziehungsweise die kartografische Abbildung der Welt verantwortlich, und zwar mit all den kritischen Aspekten, die eine solche Machtverschiebung in Bezug auf die Karte mit sich bringt. Hinter diesen neuen Kartograf_innen stehen die Monopolisierung und Zentralisierung von Daten durch Google (siehe auch Vaidhyanathan 2011). Der US-amerikanische Informatiker und Künstler Jaron Lanier weist auf diese Machtakkumulation hin, für welches er den Begriff Big Five - Google, Apple, Facebook, Amazon und Microsoft - prägte (vgl.Lanier 2014); andere sprechen auch von GAFA: Google, Apple, Facebook, Amazon (siehe z. B. Foer 2018; Zuboff 2018 u. a.). Insbesondere Google und Facebook geraten immer wieder durch die Verletzung der Privatsphäre sowie den Verstoss gegen Urheberrechte und geistiges Eigentum in die Kritik.

5. Durch den Einsatz von ortsbasierten Technologien können Informationen personalisiert und kontextualisiert werden. Vor Web 2.0 wurde mittels Hyperlinks durch das Internet navigiert. Mit der Digitalisierung beziehungsweise dem digitalen Umbruch hat sich die Nutzung sowie die Produktion von visuellen Kulturen jedoch stark verändert. War visuelle Kommunikation früher zumeist physisch und zweidimensional, ist sie heute meist digital und räumlich verortet. Das heisst, der Nutzer bekommt zugleich ein Bild von der Situation und kann sich, ohne den Ort zu kennen, räumlich bewegen, orientieren, lernen etc. Dadurch werden räumliche Bezüge aufgewertet und örtliche Situationen lassen sich bereits im Vorfeld erahnen. Damit einhergehend kommt es zu neuen Strukturierungs-, Navigations- und Orientierungsformen: Informationen werden aufgrund ihrer räumlichen Nähe angezeigt, ohne dass dabei nach topologischen Zusammenhängen gesucht wird. Mit Sender, Empfänger, Knoten (Sensoren) und Kanal (Wegstrecke) (vgl. Lynch 1960, dt. 2001) entstanden einst zentrale Metaphern für räumliche Navigationsstrukturen und zur mobilen Wegfindung. Interaktive Karten verknüpfen ihre Inhalte mit Informationen, News und Bildern als Listen, Websites und Fotografien und werden dadurch zu räumlichen Organisationen - zu einem GeoWeb -, zu visuellen Epistemologien. Über Dienste, Netzwerke und Applikationen vermag der physische Ort mit persönlichen Notizen, Geschichten und Bildern der Nutzer ${ }^{14}$ beschriftet werden (Inscribing und Tagging). Ausgehend von der künstlerischen Praxis rund um lokative Medien (Locative Media), werden diese Praktiken mittlerweile auch in sozialen Netzwerken angewandt. Karten werden zu visuellen Formen von Wissensproduktionen, die Welterfahrung pro-

14 Im Folgenden wird im Kontext mit Software und/oder Hardware der neutrale Begriff Nutzer verwendet. 
duzieren, erweitern und verständlicher machen oder dies zumindest suggerieren.

6. Eine wichtige Eigenschaft von digitalen Technologien ist deren Mobilität, Netzwerkbezug und Lokalität. Mit dem mobilen Internet sind neue räumliche Praktiken entstanden: standortbezogene Kartendienste (location-based Services) wie Google Maps, Google Earth und OpenStreetMap (OSM) oder räumliche soziale Netzwerke wie TwitterMap, Geochirp und Foursquare. Diese Dienste, sozialen Netzwerke und Produkte gestalten das räumliche Umfeld, in welchem wir leben, und strukturieren es um. Wir bewegen uns zunehmend in einem hybriden Raum, der sich zwischen dem physischen und dem virtuellen Datenraum befindet. Durch diese Dienste und Netzwerke kann der physische Raum mit Informationen beschrieben beziehungsweise mit raumbezogenen Daten überlagert werden.

7. Eine der einflussreichsten Technologien (emerging Technologies) und innovativsten Praktiken ist Augmented Reality (AR). Mit dem zu erwartenden Durchbruch von Augmented Reality werden kartografische Produkte begünstigt. Spezifische Applikationen, die uns bei alltäglichen Aufgaben wie der Restaurantsuche oder dem Anzeigen von Fahrplänen vor Ort unterstützen, wie zum Beispiel die Monokel-Funktion von Yelp, sind auf dem Vormarsch. Um auf augmentierte Informationen zugreifen zu können, braucht es neben der Darstellung der Information im physischen Raum eine (Karten-)Metapher, um einen Raumbezug herzustellen, die Daten und Informationen zu verorten und den Raum abzubilden. Karten werden dabei zu Interfaces, um abstrakte Daten und Informationen zu ordnen sowie Zugang zu innen zu gewährleisten. Die Technologie von Augmented Reality, die auf georeferenzierte Inhalte zugreift, ist für das Design deshalb interessant, weil der Datenzugriff über ein Interface erfolgt und die Information im physischen Raum dargestellt werden kann. In einem solch situierten Umfeld tun sich oben beschriebene einflussreiche Diskussionen und Perspektivierungen auf, die Design in seiner räumlichen Dimension vorantreiben. 


\section{Aufbau und Hintergrund der Publikation}

Die vorliegende Monografie ist Teil des vom Schweizerischen Nationalfonds (SNF) finanzierten Forschungsprojekts Augmented Space (2015 - 2019), das aus einer designzentrierten Perspektive erforschte, wie sich die fortschreitende Digitalisierung sinnlich-sensorisch auf die uns umgebende Welt/uns umgebenden Räume auswirkt und auswirken wird. Das Forschungsvorhaben ging von der Hypothese aus, dass in digitalen Kulturen Wissen und die Wissensproduktion räumlich organisiert, strukturiert, gebildet und rezipiert wird. Teil der Forschung war eine Exploration zur Produktion, Strukturierung und Rezeption von hybriden Wissensräumen, welche auf der Durchdringung von physischen mit digitalen Räumen beruht. Der Fokus richtete sich dabei auf die Wahrnehmung, welche diese Räume hervorbringen, und nicht auf die Technologie (spezifisch technisches bzw. technologisches Verständnis). Anders gesagt: Die kulturelle Praxis und das ästhetische Verständnis standen im Vordergrund. Die Resultate des Forschungsprojekts beinhalten praktische Erkenntnisse, die in die Augmented-Reality-Applikation Augmented Space eingeflossen sind, sowie theoretische Erkenntnisse. Diese Applikation, welche im letzten Teil der Publikation besprochen wird, ist parallel zum Verfassen des Theorieteils als exemplarische Designpraxis entstanden, dabei jedoch nicht direkt an den Text gekoppelt. Beide Teile sind im Verständnis von künstlerischer Forschung (siehe hierzu z. B. Bippus 2012; Badura et al. 2015 u. a.) gleichwertig zu betrachten und Teil der hier vorliegenden Monografie Augmented Spaces and Maps. Das Design von kartenbasierten Interfaces.

In enger Verzahnung mit dem Praxisteil und in stetiger Rückführung und Überprüfung der Konzepte und Theorien wurde der Theorieteil verfasst mit dem Ziel, die zentralen Aspekte für den weiter oben beschriebenen Raumwandel und der damit verbundenen Neuperspektivierung des Kartendesigns näher zu beleuchten. Ausgehend von der Karte als raumparadigmatisches Tool beziehungsweise der Kartografie als räumliche Disziplin werden Beispiele, Methoden, Ansätze und Kontexte diskutiert, die die Weiterentwicklung einer kartenbasierten Schnittstelle nachhaltig beeinflusst haben oder das Potenzial aufweisen, die 〈Karte der Zukunft〉 zu gestalten. In vier Kapiteln werden Themen beleuchtet, die diesbezüglich einem Wandel unterworfen sind. In Anlehnung an Gilles Deleuzes \& Félix Guattaris Tausend Plateaus (2005) werden die losen und doch rhizomatisch miteinander verwobenen Aspekte kritisch beleuchtet, weitergedacht und in weiterführende Bezüge gesetzt. Dabei sind die Trennlinien nicht scharf und die Bezüge und Beispiele können auch für andere 
Kapitel herangezogen werden. Die Inhalte sind jeweils dort eingeordnet, wo dies für die Argumentation am sinnvollsten erschien. Die vier Kapitel im Einzelnen:

Raum - der digitale Spatial Turn: Das Kapitel führt in die für den Wandel grundlegenden Raumbegriffe ein und stellt raumbezogene Technologien vor. Die Karte ist zu einem raumparadigmatischen Tool geworden und ermöglicht neue Zugangsformen zum Raum. In einem solch situierten Umfeld ist der Raumbegriff nicht länger fixiert und abhängig von dem jeweiligen Medium. Inwiefern verändern sich der physische Raum und die Raumerfahrung/-wahrnehmung? Welche Möglichkeiten bieten sich dem Design?

Interface - die Ästhetik von medialen Oberflächen: Die Betrachtung von analogen und digitalen Oberflächen, von Interfaces mit einem Kartenbezug steht im Mittelpunkt dieses Kapitels. Seit der Digitalisierung wurden die Oberflächen in Bezug auf Ästhetik einem grundlegenden Wandel unterzogen. Das traditionell analoge Kartenbild hat sich zu einer interaktiven digitalen Schnittstelle gewandelt. Wie hat sich die Kartengestalt verändert? Wie können Karten zu Schnittstellen zwischen Raum und Mensch - Computer oder System werden?

Macht - die neuen Kartograf_innen: Urheberschaft, Macht und Globalisierung im Zusammenhang mit der Kartenherstellung sind Thema dieses Kapitels. Mit der Einführung von GIS kam es zu einer Demokratisierung in der Kartenproduktion. Zugleich fand ein Wechsel von einer staatlichen Aufgabe hin zur Kontrolle durch private Internetfirmen statt. Wer zeichnet für die Kartenproduktion verantwortlich? Wer ermöglicht den Zugang zu Geodaten und wie ist das Verhältnis von Produzent_innen und Nutzern?

Navigation - die Karte kommt in Bewegung: Erörtert werden verschiedene Navigationsformen und Erzählstrukturen. Durch die Verbreitung von digitalen Karten erlebt die Navigation eine radikale Veränderung. Es wird nicht mehr nach topografischen Strukturen gesucht, sondern nach räumlichen Inhalten. Durch die veränderten Navigationsformen sind Erzählstrukturen einem Wandel unterworfen. Welchen Einfluss haben raumbezogene Technologien auf die Art und Weise, wie wir navigieren? Wie beeinflussen lokative Medien die Form, wie wir Raum erleben?

Im Praxisteil wird der Einsatz einer Augmented-Reality-Applikation im Hinblick auf die Lesbarkeit eines Orts, eines Raums untersucht. Dazu wurde das ehemalige Waren- und Zollfreilager auf dem Dreispitz-Areal in Basel, auf welchem die Hochschule für Gestaltung und Kunst FHNW angesiedelt ist, zum Forschungsgegenstand. Im Zentrum der Untersuchung standen zwei Aspekte: 1) wie sich die Nutzer 
in hybriden Räumen zurechtfinden, die sich aus der Durchdringung von physischen und virtuellen Räumen und durch die Nachnutzung des historischen Areals ergeben, und 2) was es heisst, dass eine Kunsthochschule sich auf dem Areal eines ehemaligen Zollfreilagers befindet. Das Dreispitz-Areal, eines der grössten Entwicklungsgebiete in der Schweiz, und die dortige Ansiedlung des Campus der Künste (Hochschule für Gestaltung und Kunst FHNW) stehen exemplarisch für den Wandel einer Industrie- in eine Informationsgesellschaft. Die Transformation des Areals kann als Metapher für den Strukturwandel einer materiellen - mehrheitlich aus Gütern bestehenden - Welt zu einer virtuellen - mehrheitlich aus Daten bestehenden -, fluiden, vernetzten und digitalen (Informations-)Gesellschaft gesehen werden. Eine solche Um- und Weiternutzung ist exemplarisch für drei weitere Kunsthochschulen in der Schweiz, die sich ebenfalls in ausgedienten Industrieanlagen befinden (die Zürcher Hochschule der Künste [ZHdK] in einer ehemaligen Milchfabrik im Toni-Areal, die Hochschule Luzern [HSLU] in der Viscosistadt, die Hochschule der Künste Bern [HKB] in der ehemaligen Tuchschildfabrik u. a.).

Augmented Space - am Beispiel des Zollfreilagers auf dem Dreispitz - ausgehend von den hybriden Räumen, die sich aus der ehemaligen Nutzung beziehungsweise durch den kulturellen Wandel ergeben, wird der Ort erforscht. Die Augmented-Reality-Applikation führt zu den Schauplätzen des ehemaligen Basler Zollfreilagers und lässt dessen fast 90-jährige Geschichte räumlich erleben. Die App ermöglicht eine historische Perspektive auf den Campus der Künste in Basel und kombiniert diesen Blick mit einer zeitgenössischen Kritik an der Praxis heutiger Zollfreilager mit Kunstbezug. In einer Gegenüberstellung werden Praxen rund um die Kunstfreilager, den Kunstmarkt und die Kunstsammler_innen untersucht.

Alle Inhalte gehen auf meine eigene langjährige Unterrichts- und Forschungserfahrung zurück, unter anderem an der Hochschule für Gestaltung und Kunst FHNW sowie auf meine eigene Tätigkeit als Designerin im Bereich von Spatial Design und Wayfinding Systems. Zentral für die Entstehung dieser Monografie waren insbesondere folgende Projekte aus Lehre und Forschung: die Lehrveranstaltungen Photogrammetry-based 3-D Modelling for VR (2017), Kosmos Dreispitz - Kultur digital erzählt (2016), Dreispitz-Areal - eine alternative Kartographie (2016), Medialisierte Räume und verräumlichte Medien (2015) sowie das Forschungsprojekt $A$ Journey to Palmyra (2018). ${ }^{15}$

15 Nähere Informationen zu den Lehrveranstaltungen wie auch zum Forschungsprojekt finden sich im Anhang. 
Theorie 

Raum

Der digitale

Spatial Turn 


\section{Der Raumwandel in digitalen Gesellschaften}

Der Spatial Turn ${ }^{16}$, der von den Geisteswissenschaften ausging, war in den 1980er-Jahren vor allem eine Frage des Orts. Im Zuge des digitalen Umbruchs verändert sich ein solcher Raumbegriff. Die diskutierte Raumordnung nach Topologie (Ort) und Zeit (Distanz) wurde aufgelöst. Mit dem GeoWeb sowie den Möglichkeiten digitaler Praktiken (wie Verortungstechnologien, Georeferenzierung u.a.) wird Raum auch medial konstruiert, in Beziehung gebracht und inhaltlich beziehungsweise kritisch strukturiert. Hierfür ist eine Unterscheidung zwischen Ort und Raum (Michel de Certeau), also zwischen Daten und Codes, welche Räume hervorbringen (Lev Manovich; Rob Kitchin \& Martin 
Dodge), notwendig. Diese Konzepte,

die auch für die Designdiszipli-

nen interessant sind, werden im

Folgenden noch näher erörtert.

Durch das Raumparadigma hat sich die Raumproduktion (Henri

Lefebvre) zunächst auf die Raumerweiterung verlagert. Mit Web 2.0 (und dem Mobil-Werden von Daten) kommt es zu einer Raumdurchdringung. Dies führt zu einer Koproduktion von MenschMaschine und Raum. Es entstehen neue Räume, die nur im Zusammenspiel von Raum, Nutzern und Software produziert werden können. Damit sind konstitutive Elemente eines digitalen Spatial Turn geschaffen: Der Raum wird von Nutzern und Software konstruiert, erweitert oder durchdrungen. Mit solchen Überlegungen - zusammen mit der zunehmenden Konvergenz von digitalen Medien und Raum verschiebt sich die Wahrnehmung 
etwa auf einen sogenannten Media Spatial Turn (vgl. Döring \& Thielmann 2009b), Geomedial Turn (vgl. ebd.) oder Digital Turn (vgl. Junge 2012). Diese Bezeichnungen umkreisen alle dasselbe Phänomen: die Verschmelzung eines physischen Orts mit einem standortbezogenen Dienst im weitesten Sinne. Durch diese Raumproduktion entstehen neue Praktiken visueller Ästhetiken und Epistemologien. Konstitutiv hierfür sind Karten und kartografische Dienste, die den Raum erweitern und durchdringen. Karten und kartografisches Wissen sind deshalb ein wichtiger Ausdruck von Raum, der räumlichen Dimension. 


\section{Der Spatial Turn}

Als Ende des 20. Jahrhunderts Satellitenbilder öffentlich zugänglich wurden, führte dies zur These vom ، Verschwinden des Raums, (Paul Virilio). Gleichzeitig wurde von Nigel Thrift die Ausdehnung des erlebten Raums festgestellt, in welchem sich der Lebensraum der Erdbewohner_innen um ein Vielfaches potenziert. Geografische, kommunikative und kulturelle Grenzen werden überbrückbar; alles rückt näher zueinander, gleicht sich in ständigem Austausch einander an. Ende der 1980erJahren beginnt man, neu über Raum nachzudenken. Wie Krygier und Wood ausführen, können geografische Muster dazu anregen, Dinge anders zu sehen: "Maps don't simply locate things: they can lead us to insights, discoveries, and new ways of understanding. We see geographic patterns, and those patterns may lead us to think about things in a new way." (Krygier \& Wood 2005: 8) In der Leitdisziplin Humangeografie wird von einem Spatial Turn gesprochen (vgl.Dünne \& Günzel 2006; Dünne 2008; Döring \& Thielmann 2009b; Günzel 2009; Döring 2010; Günzel 2017), wenn die Erkenntnis räumlich ist, das heisst, wenn sie methodisch zur Verräumlichung führt. Die Wortgeschichte des Begriffs Spatial Turn / siehe auch Bachmann-Medick 2010; Günzel 2010) geht auf den Humangeografen Edward W. Soja zurück, der ihn 1989 erstmals in seinem Buch Postmodern Geographies verwendet (Döring \& Thielmann 2009b: 7 f.). Die Bezeichnung wird dort jedoch nicht eingeführt, sondern eher beiläufig zur Umschreibung einer räumlichen Wende in dem

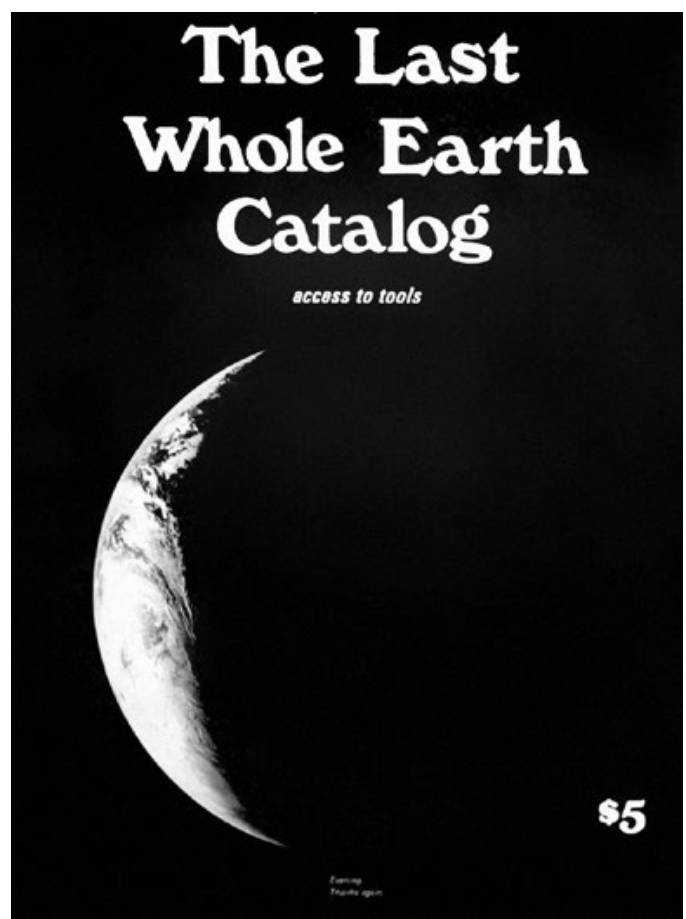

Mit Blue Marble-Serie der NASA (1972), welche auch als Cover für verschiedene Ausgaben des Whole Earth Catalog verwendet worden ist, wurde erstmals auf visuelle Muster wie zum Beispiel den Klimawandel aufmerksam gemacht. 
Artikel “Des Espaces d'Autres» des französischen Philosophen Michel Foucault (1926-1984) gebraucht (1967 [Vortrag] bzw. 1984, dt. Ausgabe: Von anderen Räumen [2006]). Mit der Wiederentdeckung des Raums als Forschungsparadigma werden neue Perspektiven auf die Raumforschung, Raumkonzepte und Raumproduktionen möglich. Den Ansätzen in den Kultur- und Sozialwissenschaften gemeinsam ist ihr konstitutiver Charakter, der Räume nicht als gegebene Grösse oder Behälter versteht, sondern sie aus Prozessen und Handlungen hervorgehen lässt. Der Raum wird als soziales Konstrukt, als Austragungsort kreativen Schaffens und als Ort des Diskurses verstanden. Dadurch werden Räume zu verschiedenen Ebenen, die Menschen und Dinge zusammenbringen und ihre Beziehungen zueinander aktiv gestalten.

Mit der Abwendung von einer Raumauffassung als Behälter beziehungsweise mit der Loslösung des Raums aus der starren Metapher eines Containers (Newton'scher Raum) findet eine Bedeutungsverschiebung zu einem flexiblen, dynamischen Raumgebilde statt. Ein solcher Raum tritt besonders als visueller Raum hervor, in welchem verschiedene Ereignisse stattfinden können. Begreift man Raum nicht als etwas Konstantes, als etwas Gegebenes, sondern als soziales Produkt, steht "das agierende Subjekt, der soziale Akteur im Zentrum " (Werlen 2008: 383). Der physikalische Raum ist bestimmend für subjektives Handeln, während der wahrnehmbare Raum ein Produkt von nicht objektiv messbaren Handlungen und Wahrnehmungsphänomenen wird (zum Begriff von Wahrnehmung und Atmosphäre vgl. Böhme 1995, 2006; Lehnert 2011 u. a.). Der Raum wird einerseits zum Medium der Darstellung, der Veranschaulichung. Dieser Anschauungsraum gibt Auskunft über die Anordnung und Erscheinung von Dingen, Menschen und Konstellationen, die sich darin befinden. Andererseits wird der Raum zum Handlungsraum, den wir durch unsere Anwesenheit beeinflussen und hervorbringen können. Dieser Handlungsraum entsteht im Anschauungsraum und wandelt sich durch Bewegung zu einem dynamischen, interaktiven Raum. Damit rückt auch in der Kunst und in der künstlerischen Forschung (Artistic Research) der Raum sowie der Raumbezug in den Fokus forschender und epistemischer Betrachtungen (vgl. Akademie der Künste 2005; Avanessian \& Hofmann 2010; Badura et al. 2015 u. a.). Mit der Digitalisierung beziehungsweise mit Web 2.0 und dem Mobil-Werden von Daten, wird der Raum zu einem Ort, in dem Dinge passieren, der Menschen und Dinge sowie Prozesse und Daten in Beziehung setzt. "Der digitale Raum der Netze überlagert den physischen Raum nicht länger oder existiert zu ihm parallel, sondern beginnt ihn auch auf der Ebene der Örtlichkeiten verstärkt zu durch- 
$\mathbf{R}$ dringen.»(Abend 2013: 15) Das Geo-Web beziehungsweise die Geomedien werden zum Bestandteil alltäglicher Raumerfahrung und standortbezogene Dienste vermögen einstmals getrennte Räume miteinander zu verbinden. Physische, mediale und geografische Räume existieren nicht mehr einfach nebeneinander, sondern bedingen, überlagern und durchdringen sich gegenseitig.

In einem kurzen Rückblick soll hier zunächst an die Konzepte von Lefebvre und de Certeau, die Urväter der Raumtheorie, erinnert werden, um diese für einen digitalen Spatial Turn fruchtbar zu machen. Der französischen Philosoph Henri Lefebvre (1901 - 1991) argumentierte, dass Raum nicht existiert, sondern von Menschen hervorgebracht wird. Nach Lefebvre ist Raum die Grundlage für soziales Handeln. Der Raum ist kein Behälter, sondern besteht vielmehr aus der Relation zwischen Dingen (Menschen und Objekten). Es gibt daher auch nicht einen Raum, sondern viele. Lefebvre konzipierte drei Begriffe: die Raumpraxis sowie den empfundenen Raum (subjektiv), die Raumrepräsentation beziehungsweise den konzipierten Raum (objektiv) sowie die Repräsentationsräume und den gelebten Raum (kollektiv). Insbesondere die Raumrepräsentation, der konzipierte Raum ist in Bezug auf Karten bedeutsam. In der Fortsetzung von Lefebvres Raumkonzepten führte der französische Raumtheoretiker Michel de Certeau (1925-1986) die Unterscheidung zwischen Raum und Ort ein. Der Raum (beweglich) entsteht durch Handlung, Aktivität und Zeit, beim Ort (unbeweglich) ist die Zeit ausgeblendet. Beim Ort existieren die Dinge, also Menschen und Objekte, nebeneinander. Unter Ort wird nach de Certeau eine Ordnung verstanden, in welcher die Elemente in Koexistenz-Beziehungen aufgeteilt sind, in anderen Worten: “eine Konstellation von festen Punkten" bilden (de Certeau 1988: 218). Der Raum hingegen ist ein Geflecht beweglicher Elemente und entsteht, wenn "man Richtungsvektoren, Geschwindigkeitsgrößen und die Variabilität der Zeit in Verbindung bringt”. (de Certeau 1988: 218) Aus der Struktur des Raums entwickelt de Certeau seine sozialen Studien zum Alltag - zu den Handlungen im Raum. Entsprechend entstehen Räume aus der Zeit, durch Handlung und mit der Bewegung. Eine Strasse beispielsweise wird erst durch einen Gehenden zu einem Raum; denn die Bewegung ist konstitutiv zur Erzeugung des Raums. Mit den digitalen Räumen kommt ein neuer Aspekt hinzu: georeferenzierte Daten. Lev Manovich sieht deshalb nicht mehr nur die Unterscheidung von Ort und Raum als bedeutend an, sondern auch "die Unterscheidung zwischen Ort und der eigenen Person, Ort und Gerät, Ort und Oberfläche, Ort und Technologie». (Manovich 2009: 396) Für Rob Kitchin und Martin Dodge ist die Raumproduktion unmittelbar an die Software gebun- 
den; fällt diese aus, kann der Raum nicht produziert werden. Die Räume werden für und von den Nutzern sowie der Software produziert und gestaltet. Diese Neuperspektivierung ist insbesondere für das Design und die Designwissenschaften interessant. Es stellt sich nicht nur die Frage nach den Beziehungen in den verschiedenen Räumen (Handlungsräume), sondern auch nach der Gestalt, also wie ein solcher Anschauungsraum konzipiert und geformt ist.

Ein Spatial Turn im Design beziehungsweise in visueller Kommunikation wurde bisher marginal behandelt. Das zeigt sich unter anderem darin, dass es kaum substanzielle Literatur zum Thema gibt, die den Begriff aus einer designtheoretischen Perspektive behandelt. Erstaunlich ist auch, dass im von Michael Erlhoff und Tim Marshall herausgegebenen Wörterbuch Design: Begriffliche Perspektiven des Design (2008) die Begriffe Raum und Spatial Turn keinen Eingang fanden. Obwohl das Wörterbuch neben klassischen Begriffen auch Definitionen jüngster Designdebatten thematisiert und verschiedene kulturelle Hintergründe miteinbezieht. Diese Marginalisierung mag an der lückenhaft aufgearbeiteten Designtheorie und -praxis liegen. Im Design treten bisher vor allem praxisorientierte Fachpublikationen wie etwa Datenvisualisierung, Informationsdesign oder Signaletik ${ }^{17}$ hervor, die den Raum als Medium thematisieren. Andere Gründe sind möglicherweise in der stark disziplinären Ausrichtung von Design begründet. In verwandten Disziplinen wie in der Architektur (vgl. beispielsweise Hilger 2011 u. a.), der Szenografie (vgl. beispielsweise Howard 2009) oder den Künsten (insbesondere in der raumspezifischen Kunst, z.B. Lammert 2005; Flügge/Kudielka/ Lammert 2007 u. a.) finden sich jedoch Raumtheorien. Mit der Durchlässigkeit der Disziplinen beziehungsweise mit den interdisziplinären Studiengängen finden raumtheoretische Konzepte allmählich Eingang in das Design und können für die Disziplin fruchtbar gemacht werden. Aufgegriffen werden derlei Konzepte bisher vor allem durch die Computerwissenschaften mit Mensch-Computer-Interaktion (HCI) und Mensch-Maschine-Interaktion (MMI), in welchem Design in Form von benutzergerechter Gestaltung von Kommunikationstechnologien verhandelt wird und Richtlinien für User Experience, Usability und Interaction Design empfohlen werden. Diese aus der Psychologie, der Ergonomie sowie der Informatik hervorgegangenen Konzepte haben die Entwicklung neuer Designansätze vorangetrieben. Gui Bonsiepe hat im Rahmen eines Vortrags auf «den Nachbarschaftscharakter der verschiedenen Disziplinen Design, Informatik und Ingenieurswissenschaften " hingewiesen und "eine ge-

17 Signaletik ist ein vor allem in der Schweiz gebräuchlicher Begriff, der Leit-, Signalisations- und Orientierungssysteme beinhaltet (engl. Wayfinding). 
$\mathbf{R}$ meinsame Disziplin des Entwerfens - eine Entwurfswissenschaft skizziert, die den Entwurf unabhängig von seiner materiellen Manifestation als Erkenntnisobjekt versteht ". (Bonsiepe 2014, zit.n. Denzinger 2018: 11) Designtheoretische Untersuchungen, die sich explizit mit dem Raum beziehungsweise mit Augmented Reality als Raumerweiterung befassen, sind mir nicht bekannt. Als Beispiel einer Designpraxis ist der amerikanische Designer Zach Lieberman zu nennen, der sich unter anderem mit der Technologie von Augmented Reality sowie neuartigen räumlichen Interfaces beschäftigt. Seine Augmented-Reality-Installation Sound AR (2017), welche Schallwellen mit Augmented Reality kombiniert und im Raum abspielbar macht, war kürzlich im Zürcher Museum der Digitalen Künste (MuDA) zu sehen. Zach hat in New York die School for Poetic Computation mitbegründet und setzt sich unter anderem für kreatives Programmieren ein.

\section{Raumproduktion im Geo-Web}

Mit dem mobilen Internet beziehungsweise dem sogenannten GeoWeb folgen auf einstmals ortlose Konzepte (Global Village, vgl. Marshall McLuhan und Bruce R. Powers 1989) Strategien der Verortung, die den Datenraum in den Fokus rücken. "Der bisher cortlose, Datenraum wird Teil des geografischen Raumes. " ${ }^{18}$ (Hemment 2004, zit.n. Popplow \& Scherffig 2013: 277) Das Geo-Web ist ein Netzwerk aus einzelnen Knoten, deren geografische Position im Internet identifiziert und kommuniziert werden können. Das Geo-Web wurde möglich, als die Verortungstechnologie mittels GPS-Satelliten für alle zugänglich und vor allem die Positionsbestimmung genauer geworden ist. Um Teil des Geo-Webs zu sein, müssen Informationen sowohl geografisch verortbar als auch digital vernetzt sein. (vgl. Abernathy 2017: 2) Konstitutiv hierfür sind neue Verortungstechnologien wie GPS, aber auch Identifikationstechnologien wie RFID (Radio Frequency Identification), QR(Quick Response)-Codes sowie Mobilfunksysteme. Das GPS ist ein satellitengestütztes Navigationssystem, das zur Positionsbestimmung und Navigation für statische wie auch bewegliche Geräte verwendet werden kann. Das System besteht aus 24 Satelliten und fünf Bodenstationen, das alles, was einen tragbaren Satellitenempfänger trägt - unabhängig von Zeit,

18 Sämtliche Auszeichnungen und Hervorhebungen in diesem wie auch sämtlichen nachfolgend aufgeführten Zitaten wurden, soweit nicht anders kenntlich gemacht, bereits innerhalb des jeweiligen Originals verwendet. 
Ort oder Wetter -, zu verorten vermag. (vgl. Kurgan 2013: 62) Wie das Internet wurde GPS im Kontext militärischer Forschung am Defense Advanced Research, einer Behörde des US-Verteidigungsministeriums, erfunden. Als das GPS erstmals für private Zwecke nutzbar gemacht wurde, waren die Standortübertragungen noch sehr ungenau. Nicht zuletzt wegen Störungen (Rauschen) durch das Militär, für welches das System einst entwickelt worden war. Laura Kurgan setzte sich im Jahre 1995 mit der Ausstellung Usted está aqui - You are here im Museu d'Art Contemporari de Barcelona (MACBA) mit der Freigabe des GPS für die Öffentlichkeit auseinander. (Abb.6) Kurgan thematisiert die Ungenauigkeit der Technologie, indem sie die unpräzisen Standortangaben mit rechnerischen Mitteln korrigiert. “Den verrauschten Daten setzt sie damit eine errechnete Drift Correction entgegen, womit sie zugleich den militärischen Entstehungskontext des Systems thematisiert und mit seinen eigenen Mitteln dekonstruiert.) (Popplow \& Scherffig 2013: 280) Massenmarkttauglich wurden GPS-Empfänger, als Autos mit Navigationsgeräten ausgestattet wurden, die die GPS-Signale empfangen und daraus Informationen zum Standort und zur Navigation berechnen konnten. Diese Innovation löste einen Boom bei standortbezogenen Diensten wie Online-Kartenapplikationen oder bei Satellitenbildern aus; der Umgang mit Raum und Standort veränderte sich dadurch signifikant. Durch die neue Möglichkeit, den eigenen Weg und Standort mittels der Signale aufzuzeichnen, entstandenen soziotechnische Kulturprak-

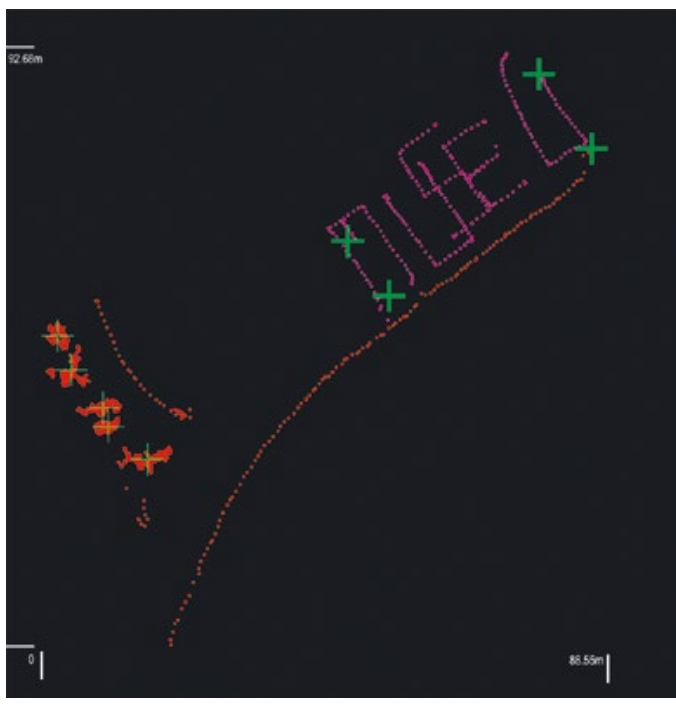

Laura Kurgan: «You are here» (1995), Nine points, two lines and five letters, stationary and mobile GPS receiver on roof above Museu d'Art Contemporani de Barcelona 
$\mathbf{R}$ tiken wie das digitale Beschriften eines Orts (Inscribing) oder das Aufzeichnen und Verfolgen eines Wegs(Tracking). Mit dem Inscribing oder dem Tracking können neue Räume produziert und kann die Bedeutung bestehender Räume verändert werden. In einem solch situierten Umfeld bilden die Räumlichkeit, die Raumproduktion sowie die Wissensproduktion neue Leitmetaphern. Dies führt zu einer neuen Raumordnung, in welcher die Beziehung von Raum und Ort, Mensch und Maschine neu organisiert werden.

Ausschlaggebend für die Raumproduktion im Geo-Web ist die Georeferenzierung. Unter Georeferenzierung wird hier die Zuweisung raumbezogener Informationen zu einem Datensatz verstanden. Der Begriff wurde insbesondere in Verbindung mit der Standortbestimmung und einem georäumlichen Koordinatensystem bedeutsam. Durch die Georeferenzierung können räumliche Daten in einem Geo-Web verwendet respektive verortet werden. Über das Geo-Web werden einzelne Punkte im Raum zu einem Bezugssystem. Der Raum wird zu einem Datenraum; der Ort zu einem Netzwerk. Das heisst, dass alles, was in einer digitalen Karte verortet ist, zum Beispiel Informationen oder Fotografien, Teil des Geo-Webs wird. Der Zugang zum Netzwerk, zum Datenraum erfolgt über das Interface. Dies gilt einerseits für die Suche von Daten über eine Suchhilfe, andererseits für die Darstellung der Daten beziehungsweise Informationen über standortbezogene Dienste. Seit Google Earth und mit der Lancierung von Google Maps sowie unzähligen Karten-Mashups und ortsbasierten Diensten werden vermehrt webbasierte Informationen an materielle Orte geknüpft. (vgl. Graham \& Zook 2013: 78) Dadurch steht zunehmend die Durchdringung von Daten mit materiellen Orten im Mittelpunkt. Die Überlagerung und Vermischung des physischen Raums mit georeferenzierten Inhalten wirken sich auf unsere Sinne und Wahrnehmung aus. Aber nicht nur unsere Wahrnehmung von Raum verändert sich grundlegend, sondern es entstehen auch neue Räume. Die von Mensch-Maschine hervorgebrachte Raumbeziehung sowie die Ubiquität von Kommunikationstechnologien im öffentlichen Raum führen zu neuen Wahrnehmungswelten. Dies gilt hierbei insbesondere für hybride Räume. Unter hybriden Räumen ist hier die Verschmelzung von einem physischen Ort mit einem digitalen Dienst zu verstehen. Hybride Räume zeichnen sich dadurch aus, dass sie sowohl Bestandteil der physischen (gebauten) Welt sowie Teil eines digitalen (fluiden) Netzwerks sind. Kennzeichnend ist auch die Verflechtung von Raum und Räumlichkeit: “Digital (or virtual) spaces, and real (physical, material, and/or actual) space."(Leszczynski 2019: 15) Mark Graham und Matthew Zook sprechen auch von “vielfachen Realitäten, die durch das in Zeit und Raum subjektive Zusam- 


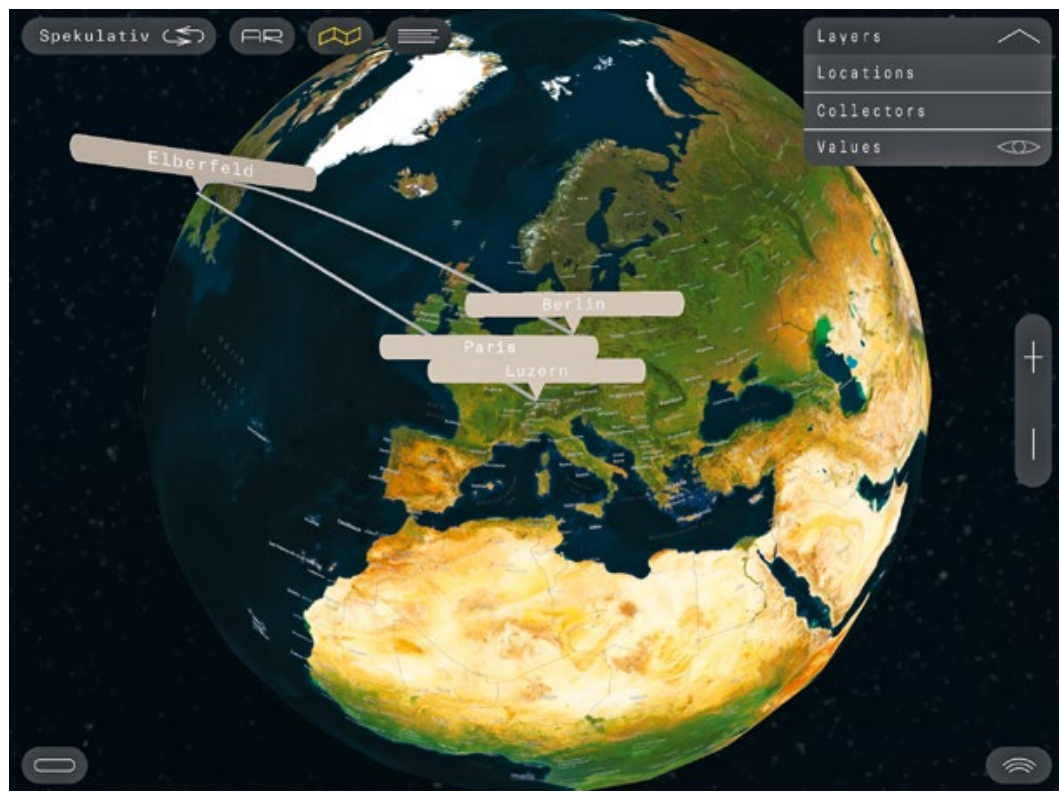

menkommen von Material und visueller Erfahrung entstehen ". (Graham \& Die AR-Applikation Augmented Space von Christine Schranz untersucht, wie verschiedene Zeiten Zook 2013: 78, Übersetzung der Autorin) Hybride Räume ermöglichen neue und Räume respektive virtuelle und materielle Erlebnisse im physischen und digitalen Raum zusammenkommen.

Formen der Orientierung, Navigation und Interaktion. Dieser Paradigmenwechsel verlangt nach innovativem Design mit ästhetisch-technologischen Konzepten. Die in diesem Zusammenhang entstandene AR-Applikation Augmented Space wird im Praxisteil näher vorgestellt. (Abb. 7) 


\section{Räumliche Analysen und Daten- visualisierungen}

Die Professionalisierung und Expertise von Visueller Kommunikation in der Kartengestaltung führen ab Mitte des 19. Jahrhunderts zu neuen Raumpraktiken in visuellen Kulturen. Anstelle topografischer Darstellungen rücken vermehrt auch räumliche Analysen und Datenvisualisierungen in den Fokus, welche ebenfalls im Zusammenhang mit dem späteren Diskurs der kritischen Kartografie bedeutsam werden. Solche Karten helfen, Zusammenhänge zu erkennen, und haben daher einen grossen Einfluss auf das Verständnis für komplexe räumliche Daten. Die Vorstellung, Karten seien objektiv geografisch exakt, steht seit längerem in der Kritik, werden sie doch von den Urheber_innen subjektiv geprägt. Seit Lefebvre geht man davon aus, dass Räume sozial produziert sind. Jason Farman führt dies folgendermassen aus: "The reading of objective space is indeed a reading, an interpretation that is never outside of the culture that produced such a reading." (Farman 2010: 876) Ein bemerkenswertes Beispiel einer räumlichen Analyse ist die Cholera-Karte des britischen Arztes John Snow (18131858) aus dem Jahre 1854. (Abb. 8) Snow fiel auf, dass die Cholera-Epidemie, die 1854 in London wütete, besonders in Soho vermehrt auftrat. Deshalb befragte er die Bewohner_innen des Stadtteils, aus welcher öffentlichen Pumpe ihr Wasser stammte, und markierte die Standorte der entsprechenden Wasserpumpen sowie jene der an Cholera

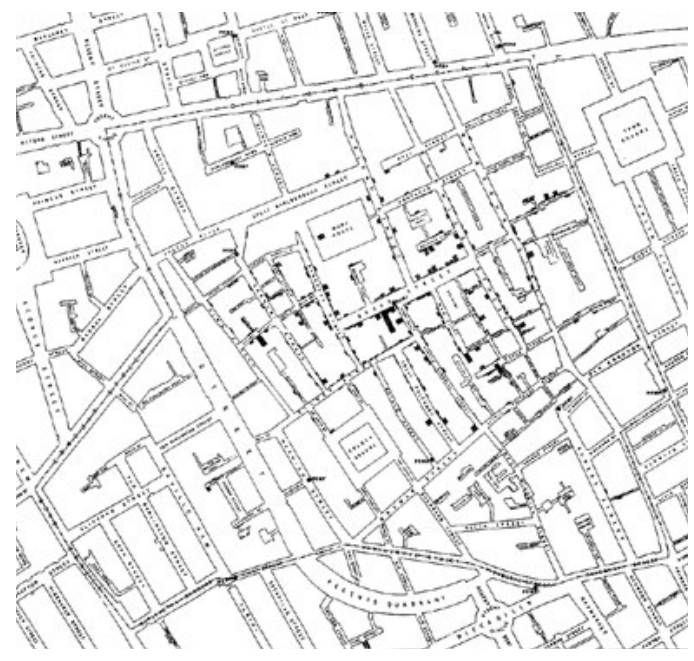

John Snow: Cholera-Karte von London (um 1854) 
Erkrankten auf einer Karte. So gelang es ihm, den Bereich, der mit dem Cholera-Ausbruch in Zusammenhang stand, näher einzugrenzen und schlussendlich in der Broad Street zu lokalisieren. Diese frühe Form von Datenvisualisierung und räumlichen Analyse war der Beginn eines Wechsels von der bis zu diesem Zeitpunkt üblichen Kartierung von topografischen Gegebenheiten hin zur Kartierung räumlicher Phänomene. Diese grafische Methode wurde von Charles Booth für seine bekannten "Poverty Maps of London" (1898-1899) übernommen sowie Anfang des 20. Jahrhunderts von der Chicago School of Sociological Research.

Mit den Satellitenbildern rückten weitere neuartige Raumpraktiken in den Fokus. Laura Kurgan vom Fachbereich für Spatial Research der Columbia Universität in New York hat erläutert, welchen signifikanten Einfluss die Bilder in Kombination mit digitalen Karten im Golfkrieg auf visuelle Kulturen ausübten: Bereits im Jahr 1991 baute Kuwait eine GIS-Datenbank auf, indem der Staat Satellitenbilder mit digitalen Karten kombinierte. Mit dieser Datenbank (der Kuwait National Database, Intergraph Corp.) vollzogen sich die militärische Intervention und der Wiederaufbau des Landes auf völlig neue Weise. Erstens war es für die USA möglich, ihre Gegeninvasion und Luftangriffe "vom Schreibtisch aus", aus weiter Entfernung zu planen. Zweitens wurde es möglich, der Öffentlichkeit Bilder in Echtzeit zu übermitteln. Und drittens nutzte der Golfstaat die Datenbank als Blaupause, um zerstörte Städte zu rekonstruieren. (vgl. Kurgan 2013: 90) Die Karte von Kuwait bestand aus “ spektralen (Farb-|Informationen der 30-Meter-Landsat-Daten mit Bodenauflösung" sowie der « räumlich (textuellen) Information der 10-Meter-Auflösung von SPOT-panchromatischen Daten. (Abb.9) Kartografische Annotationen, Symbole und Marginalien [...] gaben dem Satellitenbild einen Massstab und eine Orientierung und ermöglichten die Abstimmung mit Informationen aus einer Stadt- und Militärdatenbank bzw. mit anderen Karten.» (Kurgan 2013: 90, Übersetzung der Autorin) Wie eingangs erwähnt waren Satellitenbilder auch für den digitalen Spatial Turn paradigmatisch. Durch das Überlagern mit der geografischen Information bekommt jeder Punkt oder jedes Listenelement im Internet einen physischen Ort, ein georeferenziertes Raster von Pixeln. Dadurch können die Objekte einem geografischen Informationssystem zugeordnet und auf einer digitalen Karte sichtbar gemacht werden. Sobald lokalisiert, können Daten mit verschiedenen Elementen in Beziehung gesetzt und auf eine Karte geschichtet werden. 


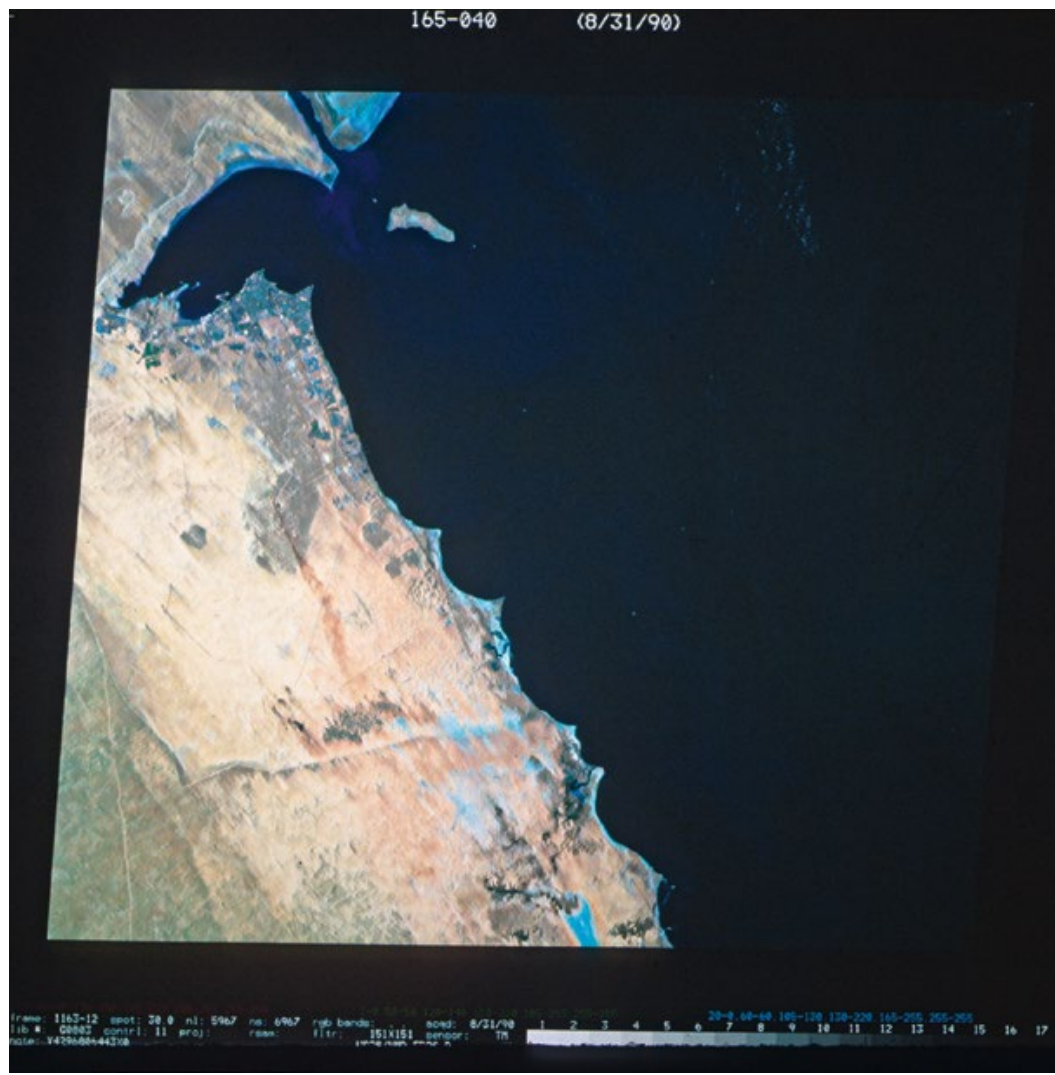

Geografische Informationssysteme haEine Satellitenaufnahme diente als Farbgrundlage ben den Raumbezug sowie das Raumfür die Karte von Kuwait.

wissen verändert. Mit GIS wurde die Erstellung von Karten auf Grundlage von auf Computern gespeicherten $\mathrm{Da}$ ten möglich. Wie Krygier und Wood aufgezeigt haben, liegt die Stärke von GIS auch in der visuellen Analyse eines räumlichen Phänomens: Jon Evans von der Sewanee - The University of the South verwendete GIS zur Analyse des Waldbestands in Tennessee. ${ }^{19}$ (Abb. 10) Durch die Kombination von Satellitendaten mit einer GIS-Kartenebene ergab die GIS-Analyse von Evans zwischen 1981 und 2003 eine Abnahme des heimischen Waldbestands um 14 Prozent und eine gleichzeitige Zunahme von

19 www.evanslab.org/impact-of-pineconversion 
Kieferplantagen um 170 Prozent. Die GIS-Analyse und die daraus resultierenden Karten liefern quantitative und visuelle Beweise für die Debatte über Waldqualität und -politik in den USA. (vgl.2005: 5) Die mögliche Interaktivität sowie dynamische Daten haben die Karte, also die Kartenproduktion, reformiert. Die Kartenherstellung entwickelte sich zu einer eigenständigen, kritischen Disziplin. (vgl.Crampton 2010: 2) Durch den technologischen Wandel war es nun - insbesondere mit webbasiertem GIS - auch Laien möglich, Karten zu produzieren. Mobile GIS-Systeme ermöglichen es den Nutzern, Karten zu verändern, indem sie diese beispielsweise um zusätzliche Informationen ergänzen. Dazu stehen bei Google Maps (wie auch bei anderen Kartenherstellern) die APIs frei zur Verfügung. Eine API-Schnittstelle ermöglicht, dass die verschiedenen Softwaredienste miteinander kommunizieren können. Durch Einbindung von APIs können auf einer für eine Website verfügbar gemachten Karte beispielsweise Daten aus Google Maps zusammengeführt werden. Karten von Google Maps können auch einfach in jede Webseite eingebunden werden, was ihre massenhafte Verbreitung begünstigt. Dies zeigt, wie radikal webbasierte GIS-Systeme wie Google Maps oder Google Earth den Umgang und Gebrauch von Karten verändert haben. Die Vorteile sind die geringen Kosten und der einfache Zugang, welcher es auch Laien erlaubt, ohne entsprechende Software und/oder Kenntnisse, Karten zu erstellen. Jedoch muss man sich auch bewusst sein, dass kartografische Produkte von Google proprietäre Sys-

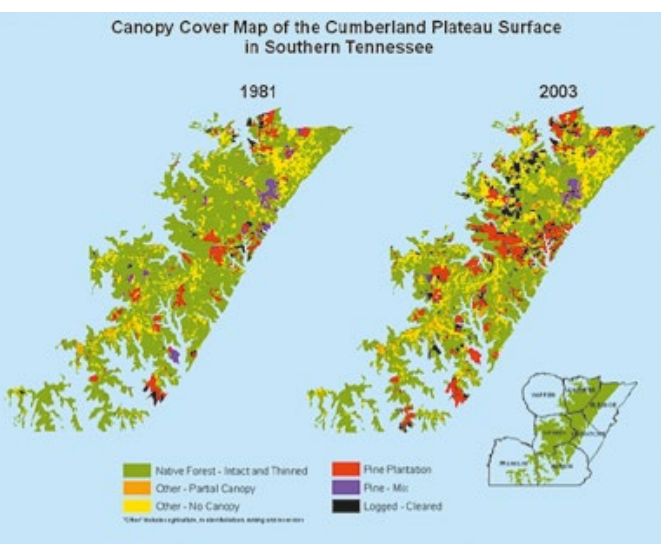

Jom Evans: Karte zur räumlichen Analyse der Veränderung des Waldbestands in Tennessee zwischen 1981 und 2003 
$\mathbf{R}$ teme (also geschlossene Systeme) sind, welche nur bedingt eine Veränderung des Kartenbilds zulassen.

Mit der wachsenden Bedeutung von georeferenzierten Inhalten beziehungsweise mit dem Geo-Web werden Karten zu Schnittstellen (Netzwerke) für das Auffinden von Informationen im Internet. Diese neuen Schnittstellen wurden mit der Möglichkeit der Lokalisierung von Person und Gerät hervorgebracht und verändern die Beziehung zwischen Nutzer, Maschinen und Raum. Darüber hinaus haben sie Einfluss auf die Verwendung von Karten: Orte werden zunehmend zu einem Netzwerk und Beziehungsgeflecht. Gleichzeitig verändert sich die Art und Weise, wie Raum und Standort genutzt und betrachtet werden. Wie ich in "Die Karte als Interface” (Schranz 2017b) ausgeführt habe, haben Satellitennavigationssysteme zur Ortung und Aufzeichnung georeferenzierter Inhalte an Bedeutung gewonnen. Obwohl das Internet durch IP- und Router-Adresse, Domainname etc. schon immer geografisch war, haben mobile Geräte den Raumbezug nochmals verändert. Der Wechsel auf mobile Geräte, die auch GPS-Tracker haben, führte dazu, dass der georeferenzierte Inhalt des Internets geografisch verortbar geworden ist. Diese Georeferenzierung hat die Karte in ein universelles Schnittstellenwerkzeug für den Zugriff auf Inhalte verwandelt. Die Gründe für den Paradigmenwechsel finden sich zum einen in der Entstehung von Geomedien und zum anderen in den neuen Möglichkeiten, die sich durch die Kombination von Open Data, Satellitenbildern und Mapping ergeben haben. Während mobile Geräte mit Verortungstechnologien vermehrt den Raum in den Fokus rücken und die Diskurse im Westen prägen, ermöglichen GPS-Tracker auch eine territoriale Zensur, wie dies beispielsweise aktuell in Saudi-Arabien oder der Türkei geschieht. 


\section{Die Raumerweiterung}

Der russisch-amerikanische Künstler und Medienkritiker Lev Manovich fasst die durch die Digitalisierung entstandenen Räume unter dem Begriff Augmented Space ${ }^{20}$ und analysiert diese aus einer kulturellen und ästhetischen Perspektive. Unter der Raumerweiterung versteht Manovich den physischen Raum, der mit dynamischen Daten überlagert wird. Dies ist einerseits der durch elektronische Anzeigetafeln und Zeichen dominierte öffentliche - spezifisch städtische-Raum, andererseits der durch tragbare Endgeräte von Nutzern konstruierte Raum. Dieser Datenraum wird über die gebaute Umwelt gelegt; die Daten können dabei ortsspezifisch oder ortsunabhängig sein und dynamische Bilder, Grafiken und Zeichen enthalten. (vgl. Manovich 2005) Die Voraussetzung einer solchen Raumerweiterung ist, dass der physische Raum durch Cellspace (Raum, der durch mobile Dienste entsteht, vgl. Bennahum 1998) oder durch elektronische Displays erweitert wird. "Sie machen den physischen Raum zu einem Datenraum, indem sie [...] [ihn] durch Daten erweitern (Cellspace, Computerbildschirme)." (Manovich 2005: 341) Die bisherige raumgebende Abhängigkeit von Orts- und Zeitgebundenheit verliert dadurch an Bedeutung. Die Atmosphäre von physischer Verbundenheit zur orts- und zeitunabhängigen Vernetzung wandelt sich. Das heisst, dass man sich nicht nur jeweils in einem physischen Raum aufzuhalten braucht, sondern gleichzeitig in mehreren digitalen Räumen anwesend sein kann.

Neben dieser Raumerweiterung durch Daten weist Manovich auch auf die Datenentnahme im physischen Raum hin. Durch verschiedene Kontrolltechniken kann jede Aktivität aufgezeichnet und Informationen können von überallher abgerufen werden. Hier zeigen sich Anknüpfungspunkte an dem von Foucault herausgearbeiteten Begriff des Panoptismus. Foucault baut seine These zum Panoptismus und zur Disziplinargesellschaft auf dem architektonischen Entwurf des Panoptikums (1787) von Jeremy Bentham auf. Das Panoptikum ist ein Konzept für den Bau eines Gefängnisses, in dem dank der architektonischen Anordnung der Räume um einen Bewachungsturm und dank des Lichteinflusses jede Bewegung der Insassen eingesehen werden kann, umgekehrt jedoch die Aufseher im Turm nicht zu sehen sind. Baute der physische Raum einst die Kontrolle auf der Grundlage von geraden Linien auf (innerhalb eines Blickwinkels), ist in modernen Gesellschaften die Überwachung nicht mehr auf das menschliche Sehvermögen angewiesen. Damit wird die Überwachung

20 Der Titel für die Publikation und Applikation wurde entsprechend hier entlehnt. 
$\mathbf{R} \quad$ weitgehend ortsunabhängig, was eine neue räumliche Logik zur Folge hat. Darauf baut ein digitales Panoptikum auf: Der «bebaute Raum [ist] Teil einer celektronischen Topologie, in der die Rahmungen der Blickpunkte und die Raster der digitalen Bilder den [...] Raum in neue Parzellen einteilen". (Virilio 2006: 262) Hier stellt sich die Frage, inwiefern in einer digitalen Raumordnung Kontrollmechanismen fortbestehen, sich formieren sowie neu manifestieren.

Der panoptische Raum schleicht sich unter anderem über Unterhaltungs- und mobile Dienste ein (z. B. Google Maps und Google Earth), wodurch sich die Disziplinargesellschaft zu einer Kontrollgesellschaft (Deleuze 1993) wandelt. Friedrich von Borries differenziert den Begriff nochmals, indem er eine Kontrollgesellschaft, die auf Freiwilligkeit basiert, unter Suggestionsgesellschaft fasst (vgl. Borries 2017: $22 \mathrm{f}$.). Dabei bezieht er sich darauf, dass die Nutzung obengenannter Serviceleistungen freiwillig ist, und wir uns der Überwachung durchaus bewusst sind, uns jedoch einreden, nichts zu verbergen zu haben, uns also sua sponte einer Überwachung und Kontrolle aussetzen. ${ }^{21}$ Der wesentliche Punkt dabei ist, dass man sich auf die Dienste von Firmen wie Google verlässt, sich damit abhängig macht und dadurch die Fähigkeit verlieren kann, selbständige Entscheidungen zu treffen, wodurch das autonome Denken und Handeln untergraben wird. Welche Auswirkungen es haben kann, sich auf standortbezogene Dienste zu verlassen, ist bekannt. Schon oft haben sich Nutzer in teils absurden Situationen wiedergefunden, als sie sich von einer digitalen Navigationshilfe haben leiten lassen. Die Abhängigkeit bezieht sich aber auch auf Daten und hegemoniale Strukturen (anstelle des physischen Territoriums). Digitale Kartenund Ortungstechnologien ermöglichen es, Personen und Objekte geografisch zu überwachen, das heisst zu verfolgen, zu markieren, wahrzunehmen und zu protokollieren, wenn sie sich von einem Ort zum anderen bewegen. “Überwachung (und Geoüberwachung) sind Formen des Wissens - zu wissen, wie viel, wo und von wem - die an Machtformen gebunden sind. "(Crampton 2010: 114, Übersetzung der Autorin) Die Souveränität zeichnet sich durch die Kontrolle von Infrastruktur, der Technologie sowie in Produktketten wie Google Maps, Streetview und Google Earth aus. Farman spricht in diesem Zusammenhang auch von einem “Digital Empire». (2010: 876)

21 Zur Akkumulation beziehungsweise zu den Prinzipen des Überwachungskapitalismus siehe auch Zuboff 2018. 


\section{Abhängigkeit von Raum und Daten}

Die britischen Humangeografen Rob Kitchin und Martin Dodge kritisieren in Code/Space (2011) die Leseweise digitaler Medien als Augmentation des physischen Raums und machen auf eine gegenseitige Abhängigkeit beziehungsweise Koproduktion von Software und physischem Raum aufmerksam. "Digital media are not just an augmentation, an optional layer over the physical city that we may or may not use. Instead, they become increasingly a constituting factor of physical space, leaving their imprint on the environment. ") Kitchin \& Dodge 2011: 220) Sie fassen diese Abhängigkeit beziehungsweise die Koexistenz von Software und physischem Raum unter Coded Space zusammen und verstehen darunter einen Raum, der nur in Verbindung mit Software funktioniert. "A space that is dependent on software for it to be transduced as intended." (Kitchin \& Dodge 2011: $261 \mathrm{f}$.) Der digitale Raum ist nach Kitchin \& Dogde also nicht ein Layer wie bei Manovich, der sich über den physischen Raum legt, sondern ist ein integrativer Bestandteil der Raumproduktion. Das heisst, beide - der digitale und der physische Raum - bedingen sich. Dabei differenzieren Kitchin \& Dodge nochmals zwischen Raum, der explizit durch die Anwendung von Programmen produziert wird (wie beispielsweise ein Check-in-Schalter am Flughafen, der bei einem Systemausfall zu einem nicht funktionierenden Ort wird), und Raum, der durch Technologie erweitert wird, aber bei Ausfall der Software noch funktioniert (wie beispielsweise eine Präsentation mithilfe von PowerPoint, die auch ohne die Software, also analog gehalten werden kann). Im ersten Beispiel besteht und wird der Raum nur aufgrund von Software produziert, fällt einer dieser Räume aus, ist die Raumproduktion nicht länger möglich. Im zweiten Beispiel haben Raum und Software keinen unmittelbaren Zusammenhang. Fast jede soziale Interaktion mit Software produziert auch einen Raum, sei es physisch im Raum oder im Geo-Web. Fällt der private Netzzugang aus oder steht kein öffentliches WLAN zur Verfügung, wird die Raumerfahrung mittels Informations- und Kommunikationstechnologien unmöglich, der Raum ist inexistent. Dies betrifft auch die Raumproduktion. Das Konzept von Code und Space hat den Raumbegriff, die Nutzung von Raum sowie die Räumlichkeit eines Orts radikal verändert. Ein Café beispielsweise, in dem kostenloser WLAN-Zugang angeboten wird, verwandelt sich zu einem Arbeitsraum und Ort sozialer Interaktion. Zusammenfassend lässt sich bisher sagen, dass für die digitale Raumproduktion nicht die Datenmenge oder Dichte der Infrastruktur ausschlaggebend ist, sondern eine wechselseitige Beziehung zwischen Daten und Raum. Erst die 
$\mathbf{R} \quad$ Software (Code) gibt dem Ort eine Bedeutung, eine Identität. Mit anderen Worten: Fällt der Code aus, verliert der Raum seine zugeordnete Bedeutung. Damit verlagert sich das Raumparadigma von der Frage, was Raum ist, hin zu der Frage, wie Raum mit Daten durchdrungen wird. 


\section{Augmented Space - Human Environment Interfaces}

Bedingt durch den technologischen Wandel - der Computer ist einerseits ubiquitär und rückt andererseits durch mobile Geräte und vernetzte Dinge (Internet of Things, IoT) in den Hintergrund - wird vermehrt der 'Raum selbst zum Interface. Augmented Reality ist eine Schnittstellentechnologie, die die reale Welt zu erweitern vermag. Das heisst, die uns umgebende physische Welt wird mit virtuellen Informa-

tionen überlagert. Die Augmentation findet meist auf dem Display eines Smartphones oder Tablets statt. Die Überlagerung kann jedoch auch als Projektion auf einer Fläche erscheinen, zum Beispiel auf einer Windschutzscheibe (HUD) oder einem Brillenglas (Google Glass). Die Welt wird dadurch nicht mehr durch die Kamera angezeigt, 
R sondern die ،Welt beziehungsweise der Raum selbst wird zum Interface,. Dadurch können wir uns auf die Umgebung konzentrieren, die lesbarer wird und wodurch es auch einfacher ist, darin zu navigieren. Ein Nachteil von tragbaren AR-Geräten (handheld $A R$ ) war bisher, dass durch die Begrenzung eines Displays der Blick eingerahmt und nie vergleichsweise immersiv wie in einer Landschaft ist. Darüber hinaus muss jeweils zwischen dem Gerät und der Umgebung hin und her gewechselt sowie (visuelle) Informationen entschlüsselt, abstrahiert und interpretiert werden. Mittels raumbasierter Medien wird nun die 'Welt zum Interface, und darüber hinaus verbindet sich der virtuelle mit dem physischen Raum. Dabei entsteht ein neuer Raum, der bereits als hybrider Raum beschrieben und eingeführt wurde. Gerade in diesen 
Zeiten paradigmatischer Entwicklungen von räumlichen Interfaces ist es unerlässlich, dass die Anwendungen nicht nur nach technischen Aspekten entwickelt werden, sondern auch benutzergerechtes Design in Bezug auf Erlebnis, Interaktion und Ergonomie mitgedacht wird. Für die Schnittstelle zwischen Mensch-Computer und Umgebung (Human Environment Interfaces) sind neue Interaktions- und Darstellungsformen bedeutsam. Der Raum wird zur medialen Schnittstelle, in welchem sich die Nutzer bewegen und verorten können. Dazu braucht es Raummetaphern, um die Information mit dem Ort zu verknüpfen und die Daten darzustellen. Um den Anstieg von vernetzten Personen-, Daten- und weiteren Informationen in ein Interface zu integrieren, ist es ratsam, die Karte als Raummetapher 
einzuführen. Die Karte wird zum

Teil des Interfaces, auf dessen Grundlage die Erschliessung des Raums stattfindet. 


\section{Augmented Reality}

Augmented Reality (AR) wurde - wie auch viele andere bedeutsame Technologien - ursprünglich für einen militärischen Kontext entwickelt. Laut Oxford English Dictionary ist AR eine Technologie, welche dem Nutzer eine kombinierte Ansicht von realen und computergenerierten Bildern oder Modellen ermöglicht. Erste AR-Anwendungen waren Head-Up Displays (HUDs) in Kampfjets, die zum Beispiel die Höhe, die Geschwindigkeit oder Navigationslinien auf die Scheiben des Cockpits projizierten. Mit der Erfindung von Virtual Reality erweiterte Ivan Sutherland um 1968 die von Douglas Engelbart “erschlossene zweidimensionale Matrix der Ein-/Ausgabemöglichkeiten, die noch stark an Texten, statischen Grafiken [...] orientiert war, zu einem nahezu multisensorischen Designraum ". (Hellige 2008: 46) Das Designparadigma wurde zunächst auf die virtuelle Umgebung (Virtual Reality) ausgeweitet. Mit den technologischen Entwicklungen kam es später zu einer Gegenströmung in Richtung Mixed Reality (vgl.Milgram \& Kishino 1994; “ein Kontinuum zwischen virtuell-digitalem und physisch-analogem Raum ” [Denzinger 2018: 8]), einer Vermischung der physischen Umgebung mit virtuellen Zeichen beziehungsweise Augmented Reality - der Erweiterung der Realität mit virtuellen Zusatzinformationen. Das Prinzip des Augmentierens wurde auf weitere Anwendungen übertragen, vor allem auf die Wartung und Bedienung komplexer Maschinen. Mit der Lancierung des Smartphones wurde der Einsatz von AR massentauglich. Fortan wurde mittels Kamera und Sensorik wie GPS, Gyroskop und Accelerometer auf dem Display des Smartphones oder Tablets augmentiert. Mit einem Augmented-Reality-Browser für mobile Geräte kann über die eingebaute Kamera die physische Welt nahezu in Echtzeit mit standortspezifischen Daten und Informationen überlagert und erweitert werden. Die auf dem mobilen Gerät laufende Software erkennt die Umgebung und ergänzt diese mit visuellen, akustischen und/oder interaktiven Informationen (wie Bild, Text, Ton oder Animation). Dabei greift das Gerät auf dynamische oder statische Daten sowie Informationen zurück und zeigt diese auf dem Display an. Das heisst: Die physische Umgebung wird mit Informationen und standortbezogenen Inhalten versehen. Der Vorteil von AR-Applikationen ist die Erweiterung der physischen Welt mit Daten respektive Informationen, die über verschiedene Sinne wahrgenommen werden, sowie die individuelle Anpassung von Information auf persönliche Bedürfnisse. Ein Nachteil ist die Begrenzung des Displays und die fehlende Darstellung der augmentierten Objekte in Blickrichtung. 


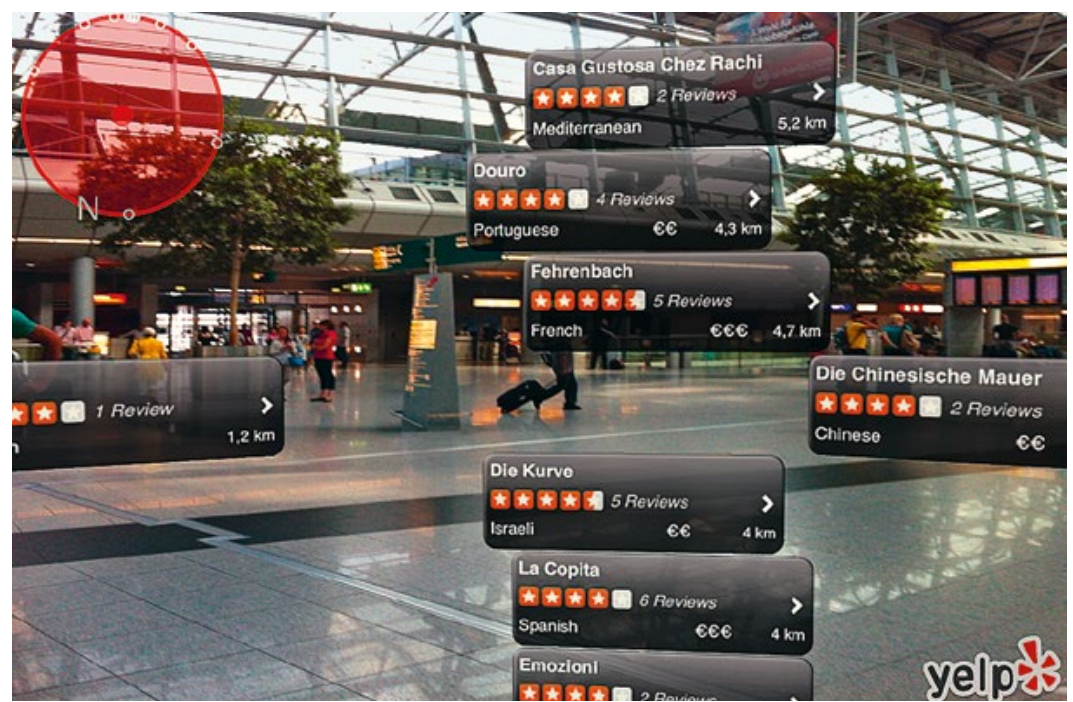

Eine der ersten standortbezogenen Augmented-Reality-Anwendung ist die Mit der Augmented-Reality-Applikation Yelp mit Monokel-Funktion werden die in der Nähe Applikation Yelp (2004). (Abb. 11) In Reviews im Raum angezeigt. vorhandenen Restaurants und die dazugehörigen Anlehnung an die Gelben Seiten zeigte die Applikation Restaurantempfehlungen in der nahen Umgebung. Mit Yelp Monocle (2009) wurde es zusätzlich möglich, Standorte mit eigenen Notizen zu versehen und Bewertungen anderer Nutzer zu lesen.

Allgemeine Bekanntheit dürfte Augmented Reality mit dem Spiel Pokémon Go im Sommer 2016 erlangt haben. In dem Spiel können Fantasiewesen eingefangen werden, die in die physische Umgebung der Nutzer augmentiert sind. Obwohl Augmented Reality noch nicht Einzug in den Massenmarkt gehalten hat, wird sie als eine der grossen Technologien der $\mathrm{Zu}-$ kunft gesehen. Um sich am Markt zu etablieren, braucht es nachhaltige und langfristige Marktabsätze (mindestens 50 Millionen Nutzer). Diese Zahl wurde mit Pokémon Go zwar erreicht, doch der Hype dauerte nur kurze Zeit an. Gemäss Studien (vgl.z. B. Markets 
and Markets $^{22}$ ) soll der Durchbruch kurz bevorstehen. Für das Jahr 2020 wird für die Bereiche Augmented Reality und Virtual Reality ein Absatz von rund 150 Milliarden US-Dollar prognostiziert, davon 120 Milliarden US-Dollar alleine für Augmented-Reality-Anwendungen. Mit der Einführung der Technologie für den Massenmarkt rücken designspezifische Fragen nach Ästhetik, Interaktion und Anwendungsszenarien in den Vordergrund. Je überzeugender das Design der Anwendungen ausfällt, desto schneller wird sich die Technologie am Markt etablieren können. Wenn der Einsatz von AR auf Smartphone oder Tablet für die User Experience in der Wegfindung oder zum besseren Verständnis eines kulturellen Erbes einleuchten dürfte, ist die Rechtfertigung einer Datenbrille zur Personenerkennung schon schwieriger. Die Datenbrille (Head-Mounted-Display) verfügt über ein integriertes Interface mit Mikrodisplay, Kamera und Spracheingabe, welches die augmentierten Informationen abzurufen vermag und auf dem Brillenglas einblendet. Kritiker_innen jedoch verweisen in diesem Zusammenhang zum einen auf den Datenschutz und die daraus resultierenden ethischen Problemen, zum anderen aber auch auf In- und Ausschlüsse durch den Besitz der Brille sowie durch die Auswahl der Informationen. Auch machen sie auf mögliche gesundheitliche Probleme wie Schwindel, Überforderung oder Desorientierung beim Gebrauch aufmerksam. (siehe u. a. Greenfield 2018: $76 \mathrm{ff}$.) Für einen Massenmarkt vorangetrieben wird die Technologie vor allem von Google mit Google Glass. Nachdem dies zunächst ein grosser Hype schien, wurde jedoch schon bald Kritik betreffend Daten- und Privatsphäre laut. Die Brille verschwand von der Bildfläche, steht allerdings, so wird spekuliert, nach wiederholten Verzögerungen in Entwicklung und Produktion derzeit erneut vor einem Comeback. ${ }^{23}$ Mittlerweile ist nun mit der HoloLens von Microsoft auch ein Konkurrenzprodukt auf dem Markt. Die HoloLens zielt jedoch eher auf Profi-Anwendungen ab; so hat sie beispielsweise speziellere Hardware zur Positionierung und Orientierung. Google Glass dagegen, welche auch ein weniger auffälliges Design hat, ist eher für den alltäglichen Gebrauch gedacht. Die Datenbrille, mit welcher die Technologie von Augmented Reality für den Endverbrauchermarkt vorangetrieben wird, ist aus der Perspektive des Designs deshalb interessant, weil die Information im Gesichtsfeld erscheinen und das im Raum sein, viel stärker wahrgenommen wird als bei einer Anordnung der Daten auf einem Bildschirm. Dadurch erfährt die Technologie eine Verschiebung zu Spatial Design und dem Begriff der

22 www.marketsandmarkets.com (abgerufen am 04.10.2018).

23 www.googlewatchblog.de/2019/03/google-glass-comeback-sieben (abgerufen am 08.04.2019). 
$\mathbf{R} \quad$ Immersion. Unter Immersion wird hier “das sinnlich-räumliche Wahrnehmen, die Auflösung von Distanz sowie das Teilhaben und -werden von Erlebnissen verstanden ". (Schranz 2013: 84) Insbesondere in Kombination mit anderen Wearables wie Google Smart Lens oder Google Watch werden hohe Summen in Forschung und Entwicklung investiert, um die Idee von der Verschmelzung von Körper und Maschine beziehungsweise dem Konzept von Cyborgs genauer zu kommen. Eine Vermutung, die im Folgenden näher beleuchtet werden soll, ist, dass Augmented Reality einen grossen Einfluss darauf hat, wie wir mit Raum umgehen, als auch, wie wir uns durch Räume bewegen, navigieren und orientieren und Informationen aufnehmen und verarbeiten. Mit Virtual Reality und Augmented Reality als Spatial-Computing-Technologien werden interessante Designperspektiven für räumliche Anwendungen möglich.

Streng genommen spricht man nur bei der Darstellung virtueller Objekte von Augmented Reality. Nach heutiger Auffassung wird darunter jedoch auch die Erweiterung von physischen Räumen und Objekten durch textliche Informationen verstanden. Wird mit Text augmentiert (Bezug mit 2-D-Objekt), spricht man von Augmented Reality im weiteren Sinne. Zentrale Aspekte von Augmented Reality sind die Überlagerung eines virtuellen und/oder physischen Raums, die Interaktivität sowie die Ausweitung der Sinneswahrnehmung. Die Augmentation erfolgt durch Grafiken, Töne und multimediale Elemente, die über die Umgebung gelegt werden und in Echtzeit mit dieser verschmelzen. Es gibt verschiedene Techniken und Möglichkeiten zu augmentieren: von der Überblendung auf dem Display bis hin zur Projektion in den Raum. Bei dem Augmentieren mit einem Smartphone oder Tablet sehen die Nutzer die Information auf dem Display des mobilen Geräts (Handheld Devices). Die physische Welt wird durch die Kamera des Geräts betrachtet und mit virtuellen Informationen überlagert. Bei der Überlagerung mittels einer Projektion wird die Information auf einen transparenten Screen projiziert. Diese Art des Augmentierens wird besonders in der Automobilbranche häufig eingesetzt. Zum Beispiel um die Navigationshilfe auf die Windschutzscheibe zu projizieren. Die augmentierte Information kann aber auch in den Raum oder auf Objekte projiziert werden. Bei allen bisherigen Technologien (Brille, Smartphone o. Ä.) ist das Hauptproblem die Erkennung des Umfelds sowie die Lokalisierung des Geräts. Für exakte Anwendungen wird deshalb heutzutage ein Verfahren zur simultanen Positionsbestimmung und Kartenherstellung genutzt, genannt SLAM (Simultaneous Localization and Mapping). Hierbei wird anhand der Bewegung über das Kamerabild die Umgebung in 3-D gescannt. AR Kit für iOS (Apple) und $A R$ Core für And- 


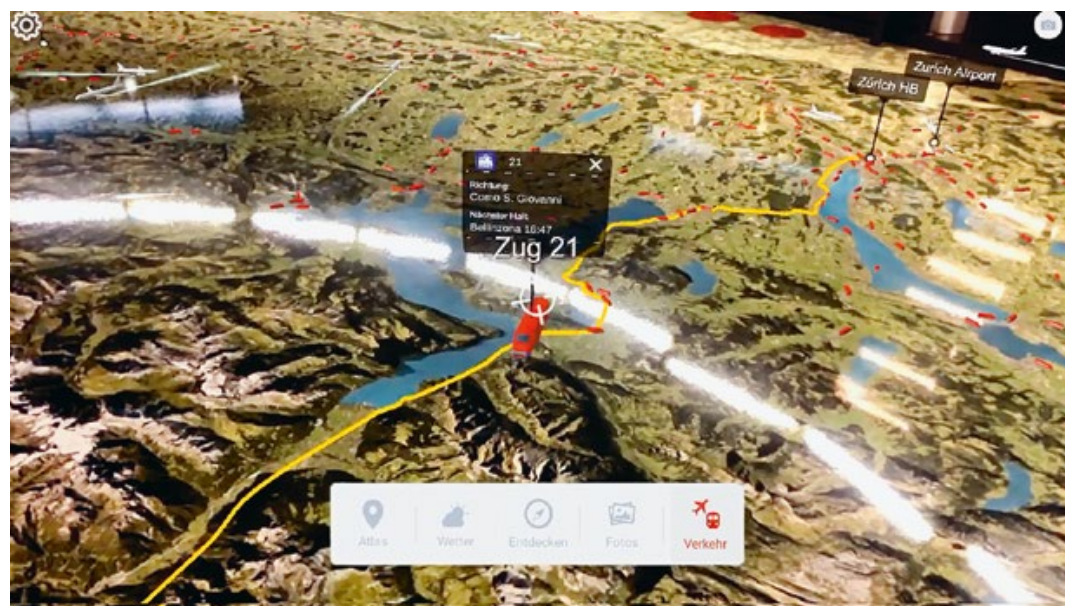

roid (Google) nutzen diese Technologie schon sehr stark. Ground Plane von Vuforia funktioniert auf ähnliche Weise. Für Anwendungen, bei denen keine Die in Zusammenarbeit mit dem Verkehrshaus der Schweiz entstandene AugmentedReality-Applikation LiveMap zeigt anhand eines begehbaren Luftbilds die Zug- und Flugexakte Positionierung notwendig ist, bewegungen in der Schweiz.

reicht eine Geo-Positionierung mit GPS, Kompass oder Gyroskop.

Durch den Einsatz von Augmented Reality wird der physische Raum um einen virtuellen Raum erweitert: Es entsteht ein neuer, ein hybrider Raum. In solch hybriden Räumen wie sie bereits weiter oben beschrieben wurden - werden neue Visualisierungs-, Strukturierungs- und Ordnungsprinzipien möglich, wodurch die Gestaltung ebendieser an das Design adressiert ist. Dadurch verändert sich nicht nur die übergeordnete Raumwahrnehmung, sondern auch, wie wir die physische Umgebung um uns herum wahrnehmen, was wir sehen und erleben.

Bisher wird Augmented Reality vor allem für die Wegfindung sowie zur erweiterten Informationsvermittlung zum Beispiel in einem historisch-kulturellen Kontext verwendet. Doch aufgrund der zunehmenden Flut an Informationen und deren Komplexität wird dieser Technologie ein grosses Poten- 
$\mathbf{R} \quad$ zial vorausgesagt. Die Entwicklung des Raumerlebnisses sowie die Gestaltung von räumlichen Schnittstellen werden künftig zum Aufgabengebiet von Designer_innen gehören. Das Design beinhaltet vor allem die grafischen Schnittstellen auf Oberflächen, die Unterstützung der Navigation, die User Experience sowie die Informationsaufnahme und -verarbeitung. Dynamische Daten und Informationen können ortsspezifisch auf den Nutzer reagieren oder in Form von personalisierten Daten um spezifische Merkmale erweitert werden, was auch kritisch gesehen werden sollte. Durch die Durchdringung des physischen Raums mit der virtuellen Welt werden hybride Räume gebildet. Diese Räume haben eine signifikante Auswirkung auf das Design von Kommunikationstechnologien. Zu denken ist hier insbesondere daran, wie Raum und Daten organisiert, strukturiert, empfangen und gebildet werden. Im Hinblick auf die Re-Aktivierung und Überlagerung von physischem mit virtuellem Raum ist Augmented Reality eine vielversprechende Technologie. Wenn in $\mathrm{Zu}-$ kunft der Blick auf den Bildschirm durch eine Datenbrille oder Linse abgelöst wird, wird sich auch das Orientierungs- und Navigationsverhalten verändern. Der gelernte Orientierungssinn, also das Orientierungsverhalten wird angepasst und erweitert.

\section{Über Augmented Reality zum hybriden Raum}

Wie ich in "Augmented Reality in Design. Thinking about Hybrid Forms of Virtual and Physical Space in Design" (vgl. Schranz 2014) herausgearbeitet habe, war es ein grosser konzeptioneller Schritt, eine dreidimensionale Umgebung in eine zweidimensionale Form zu bringen. Der wesentliche Vorteil von Augmented Reality ist, dass nicht auf eine Übersetzung des Raums zurückgegriffen werden muss, sondern die Welt durchdrungen und damit erweitert wird - ein wichtiger Aspekt, da die Raumrepräsentation (Lefebvre) in der Kartenwissenschaft schon immer folgende Frage aufgeworfen hat: Wie sollen die relevanten Informationen ausgewählt, realistisch dargestellt und geografisch wiedererkannt werden? Seitdem es Karten gibt, stehen Kartograf_innen vor der Aufgabe, die Umgebung zu generalisieren und die dreidimensionale Welt zweidimensional darzustellen. Der Einsatz von AR-Technologie für Navigationsaufgaben birgt ein grosses Potenzial. Der Vorteil liegt in der Möglichkeit, so die physische Welt mit virtuellen Informationen zusammenbringen zu können. Über die Technologie und mit Anknüpfung an eine GIS- 


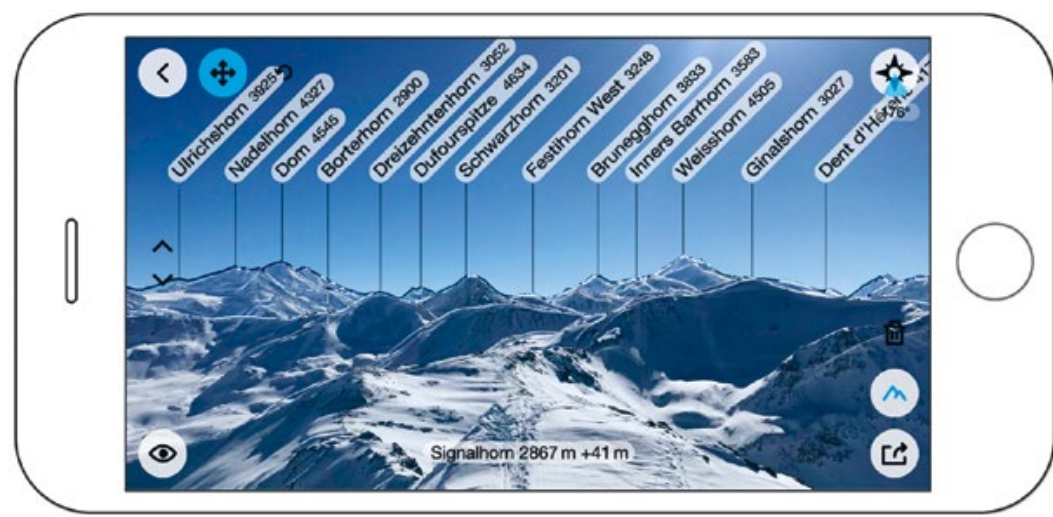

Datenbank kann die Umgebung mit nutzerzentrierten dynamischen InforMit der Augmented-Reality-Applikation PeakFinder Alps von Fabio Soldati ist ein Abgleich der Karte mationen durchdrungen und erweitert mit der physischen Welt nicht mehr nötig. werden. Die virtuellen Objekte können auf einem tragbaren Gerät, einer Windschutzscheibe oder auch in Räumen erscheinen. Somit eignet sich diese Technologie für die verschiedensten Navigationen für Fussgänger, Autos oder auch in Gebäuden. (vgl.hierzu auch Narzt et al. 2004, 2005; Bhorka 2017 u. a.) Die Gestaltung der entsprechenden Navigationsschnittstelle ist eine Herausforderung und birgt ein grosses Potenzial für den Designbereich. Die AR-Applikation PeakFinder Alps des Schweizers Fabio Soldati ist ein gutes Beispiel für einen Einsatz der Technologie mit einer gelungenen User Experience (Design: Tabea Schneider). (Abb. 13) Über das Kamerabild erkennt die App die umgebenden Berge und liefert an einem beliebigen Ort ein 360-Grad-Panorama inklusive Bergnamen. Bei vorherigem Download der Daten ist dies sogar offline möglich. Anhand des eigenen Standorts generiert die App im Umkreis von 300 Kilometern mithilfe von feinen Linien ein Bergpanorama mit Namen sowie Höhenangaben und Entfernungen zu den einzelnen Gipfeln. Die App beinhaltet 
R weltweit mehr als 250000 Bergnamen, A dazu greift sie auf GIS-Daten und Höhenkurvenmodelle von OpenStreetMap zurück.

Spannend an der Technologie von AR ist das individuelle Zusammentreffen von virtueller und physischer Realität in Zeit und Raum. AR-Anwendungen sind für die subjektzentrierte Kommunikation im (halb-)öffentlichen Raum äusserst interessant. Sie ermöglichen den Einbezug von Zusatzinformationen sowie die einfache Aktualisierung von Informationen in personalisierter oder standortspezifischer Form. Denkbare Anwendungsszenarien für den Alltagsgebrauch sind beispielsweise die - im Raum und vor Ort - Zurverfügungstellung von Informationen zu touristischen Sehenswürdigkeiten oder individualisierte Informationen wie im Raum stehende Fahrpläne oder Restaurantempfehlungen. Durch die Möglichkeit, cin der Zeit zu reisen, ist der Nutzen und Mehrwert von AR auch für einen kulturell-historischen Kontext bedeutsam. Durch die Überlagerung des physischen Orts mit historischen Bildern besteht die Möglichkeit, die Vergangenheit zu vergegenwärtigen. So lassen sich historische Welten erkunden und Reisen zu Originalschauplätzen, in vergangene Zeiten unternehmen (vgl. hierzu auch Chen \& Fragomeni 2018; Ioannides 2018 u. a. ${ }^{24}$ ). Ein Beispiel einer solchen Anwendung ist die App Streetmuseum (2010) des Museum of London. (Abb. 14) An ausgewählten Standorten in der Londoner

24 Bisherige Publikationen, die zu diesem Thema erschienen sind, fokussieren insbesondere auf technologische Aspekte und behandeln kaum die kulturelle Praxis. 
Innenstadt kann die reale Umgebung mit historischen Bildern überlagert werden. An geschichtsträchtigen Schauplätzen wird dadurch die Vergangenheit erlebbar. Ein solch räumliches Erlebnis verändert die Bedeutung eines Orts und schärft zugleich das geschichtliche Bewusstsein und die Raumwahrnehmung (vgl. hierzu auch Schlögel 2011). Ein wesentlicher Vorteil von Augmented Reality ist, dass nicht auf eine Raumrepräsentation zurückgegriffen werden muss, sondern die reale Umgebung durch Einblendung beziehungsweise Überblendung nutzerzentrierter Information erweitert wird. Deshalb birgt diese Technologie gerade im Zusammenhang mit dem Erleben und Vermitteln von auch heute nicht mehr sicht-, erfahr- und greifbarem kulturellen Erbe viel Potenzial.

\section{Raum-Mensch-Interaktion}

Mit Ubiquitous Computing (vgl. Weiser 1991) wurde erstmals das Verschwinden des sichtbaren Computers aus dem Alltag beschrieben sowie die ständige Verfügbarkeit von digitaler Informationsverarbeitung machbar. Möglich wurde dies weitgehend mit dem Internet der Dinge (Internet of Things), das kleineren beziehungsweise eingebauten Computern die Interaktion zwischen Dingen ermöglicht. Wie beispielsweise der mit dem Internet verbundene Kühlschrank, der automatisch Milch bestellt, sobald diese aufgebraucht ist. Der Designer Philippe Starck sieht im Trend der Entmaterialisierung das Ende der (materiellen Pflichten, und damit auch das Ende des traditionellen Designverständnisses (das aus den Bedürfnissen des Industriezeitalters entstanden ist und neben der Funktionalität vor allem der Repräsentation wie Status und Prestige dient). Starck sieht im zukünftigen Design vor allem die Aufgabe, alltägliche Abläufe erträglicher zu machen. Seine Vision ist eine Welt, “in der keine Materie existiert, nur Gefühl. In einer solchen Welt können Sie in einem leeren Haus wohnen, das Sie nur mit Dingen bestücken, die Ihnen gefallen: einer geliebten Person, einem Regenbogen, der durch das Fenster auf den Boden fällt.» (Starck 2018: o.S.) Auch wenn solche Ideen radikal sind und sich wahrscheinlich auch nicht so leicht umsetzen lassen (Wo schlafe ich? Womit bekleide ich mich?), ist es spannend, über den Einfluss der Entmaterialisierung auf das Design nachzudenken. Ob in einer weitgehend entmaterialisierten Welt oder nicht, die Aufgabe von Designer_innen wird es sein, den Alltag verständlich(er) zu machen. Die kleinen und zunehmend unsichtbareren Computer werden wir vermehrt auf und an uns tragen (Wearable 
$\mathbf{R}$ Computing). Anstelle der Computer A treten intelligente und vernetzte Alltagsgegenstände, tragbare Geräte und ortbasierte Dienste, die mit dem Internet kommunizieren. Da nicht mehr zwingend ein Bildschirm die Schnittstelle zum Computer bildet, besteht die Möglichkeit der Verlagerung des Interfaces in den Raum (siehe auch Richterich \& Schabacher 2011). Damit rücken anstelle der Computerbildschirme das Display (dreidimensionale User Interfaces) und Alltagsgegenstände in den Fokus. Mit anderen Worten: Design wird räumlich. Durch Projektionen in den Raum beziehungsweise auf Objektoberflächen ergeben sich neuartige Möglichkeiten im Design. Ganze Environments können durch innovative Technologien wie beispielsweise QR-Codes oder Leuchtdioden (Light-Emitting Diode) zum Interface werden. "Die Landschaft um uns herum wird zum Interface, in der sich Daten aller Art befinden, wie das Ranking verschiedener Restaurants oder Kartierungen nach einer Naturkatastrophe.» (Farman 2012: 43, Übersetzung der Autorin) Als einfache Varianten werden die Projektionen auf mobilen Endgeräten angezeigt. So können beispielsweise Häuserfassaden als Projektionsflächen genutzt werden, die über QR-Codes (per Scan oder georeferenzierte Daten, die beim Vorbeigehen vom Endgerät erkannt werden) angesteuert werden. (Abb.15) Mögliche Darstellungen zu Angeboten oder Veranstaltungen im Gebäudeinneren werden in Echtzeit dargestellt. Damit kann auf unschöne oder unerwünschte Werbung an den Aussenfassaden von Gebäuden verzichtet werden, die eine

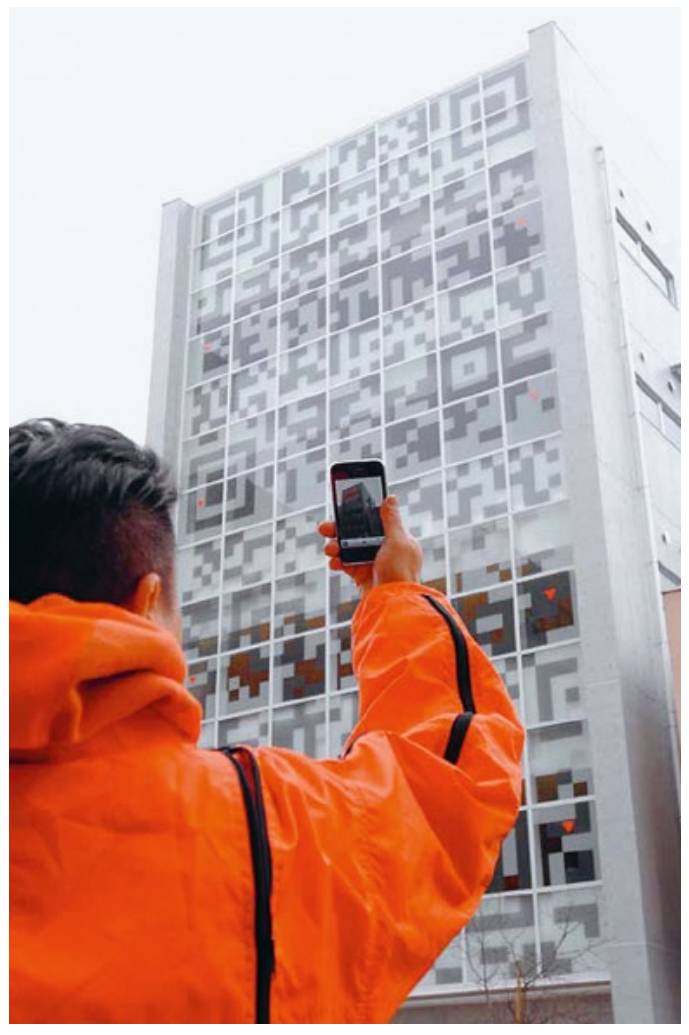

Die Fassade des $N$ Building in einem Einkaufsviertel in Tokyo ist als QR-Code lesbar. 
Dauerberieselung der Passanten verursachen und das Bild einer Stadt prägen. Einige Städte wie beispielsweise Rio de Janeiro verbieten bereits heute Werbung im öffentlichen Raum.

Eine exklusivere Möglichkeit sind transparente Leuchtdioden, die sämtliche Oberflächen zu Benutzeroberflächen verwandeln. Interessante Anwendungen finden sich in der Automobilindustrie, welchedieWindschutzscheibe zur Projektion einer Karte nutzen. SolcheHUDs, erstmals in Cockpits von Kampfjets eingesetzt, werden inzwischen auch in modernen Airlines oder Autos (z.B. bei General Motors) verwendet. Die Idee hinter HUDs ist, die Informationen direkt auf die Windschutzscheibe zu projizieren. Ein grosser Vorteil solcher Systeme ist, dass die Daten im Blickfeld der Lenker_innen erscheinen und der Blick nicht von der Strasse abgelenkt ist oder neu ausgerichtet und fokussiert werden muss (wie bei einem mobilen Gerät).Displays mit ADAS/Advanced Driver Assistance Systems)-Funktionen wie zum Beispiel zur Erkennung toter Winkel, zur Warnung vor Fussgängern oder zur Erinnerung an Ölwechsel und Anzeige der Geschwindigkeit werden mittels Lichtdioden auf die Windschutzscheibe übertragen. Auch erweiterte Funktionen wie Textnachrichten oder MP3Player können angezeigt und gesteuert werden. Ein Beispiel einer GPS-Navigationsapplikation für das Auto ist Hudway Go, das Google Maps API verwendet und diese auf die Windschutzscheibe projiziert. In Zukunft sollen HUDs die gesamte Windschutzscheibe füllen und dadurch eine erweiterte Realität vermitteln. Erste Forschungen

Dank vernetzter Technologie lässt sich das Interface mit einer Wischgeste von der Device-Oberfläche auf die Windschutzscheibe (verschieben). 
$\mathbf{R} \quad$ zu einem Interface, das nahtlos von einem Gerät zum anderen wechseln kann und dabei die gesamte Windschutzscheibe füllt, fanden am National Visualization and Analytics Center statt. (Abb. 16) Mit einer Wischgeste in Richtung Windschutzscheibe des Autos wechselt das Display vom mobilen Gerät auf die Windschutzscheibe und verwandelt diese in ein Interface (vgl. Farman 2012:9f.). Ein Vorteil eines solchen Systems ist, dass die Informationen immersiv mit der Landschaft verschmelzen. Ein weiterer Vorteil ist, dass der Computer unsichtbar und vermehrt in die Tätigkeit integriert wird. Durch die Ubiquität und mit der Vernetzung ist die autonome Steuerung sowie die Verwaltung von GIS-Daten, von Objekten, Personen und Systemen möglich geworden. Das klassische Interface verliert immer mehr an Bedeutung und es entstehen neuartige Konzepte räumlicher Schnittstellen. Wenn das Interface materiell stärker in den Hintergrund tritt, müssen neue Metaphern, Konzepte und sinnlich wahrnehmbare Darstellungen entwickelt werden. Diese neuen Interfaces erfordern ein radikales Umdenken im Design, sei dies in Bezug auf die Ästhetik oder in der Nutzung und im Erlebnis der Inhalte.

Ein heutzutage vielleicht noch futuristisch anmutendes Projekt ist AIDA (Affective Intelligent Driving Agent), ein Kooperationsprojekt zwischen Audi (Volkswagen-Gruppe Amerika) und dem MIT Massachusetts Institute of Technology (Senseable City Lab und Personal Robots Group). (Abb.17) Das Projekt verspricht dank eines intelligenten Navigationssystems und eines persönlichen Roboters ein innovatives Nutzer- und Fahrerlebnis. Das Navigationssystem erkennt sowohl die Gewohnheiten und Bedürfnisse der Lenker_innen als auch die Strecken und Umgebungen, auf welchen diese gewöhnlich fahren. Dazu wird das Fahrverhalten analysiert. Das System blendet Informationen zu Einkaufsmöglichkeiten, touristischen Zielen und aktuellen Events sowie dynamische Verkehrsdaten auf der Windschutzscheibe ein. Eine mögliche Aufgabe von AIDA ist beispielsweise, das Ziel und die bevorzugte Route vorherzusagen. Dies ermöglicht Anpassungen, sollte die gewohnte Route einmal nicht passierbar sein. AIDA bietet darüber hinaus eine Schnittstelle, über die Lenker_innen auf weitere Informationen zugreifen können. Um die virtuelle Erweiterung näher in die Blickrichtung zu bringen, nutzt AIDA neben der Windschutzscheibe auch die ungenutzten Bereiche des Armaturenbretts. Dadurch entsteht ein immersiver Fahrraum, in welchem das Fahrzeug, die physische Welt sowie Lenker_innen zu einem Fahrerlebnis verschmelzen. Eine grosse Herausforderung für das Design wird sicherlich sein, die Informationen so anzuordnen und darzustellen, dass die Lenker_innen nicht überfordert und abgelenkt werden. 

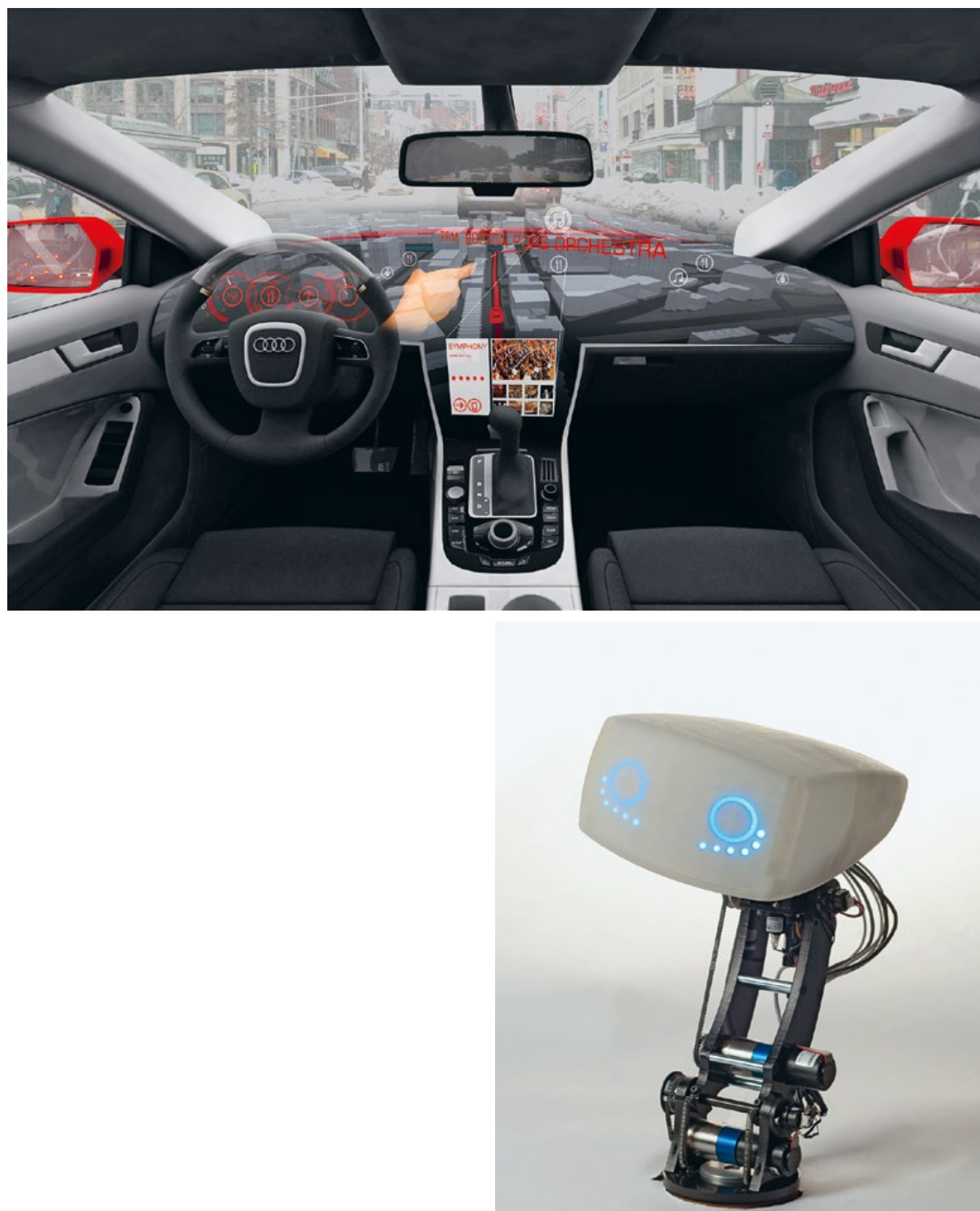

AIDA ist ein intelligentes Navigationssystem mit erweitertem Armaturenbrett und Bordcomputer. 

Interface

Die Ästhetik

von medialen

Oberflächen 
2

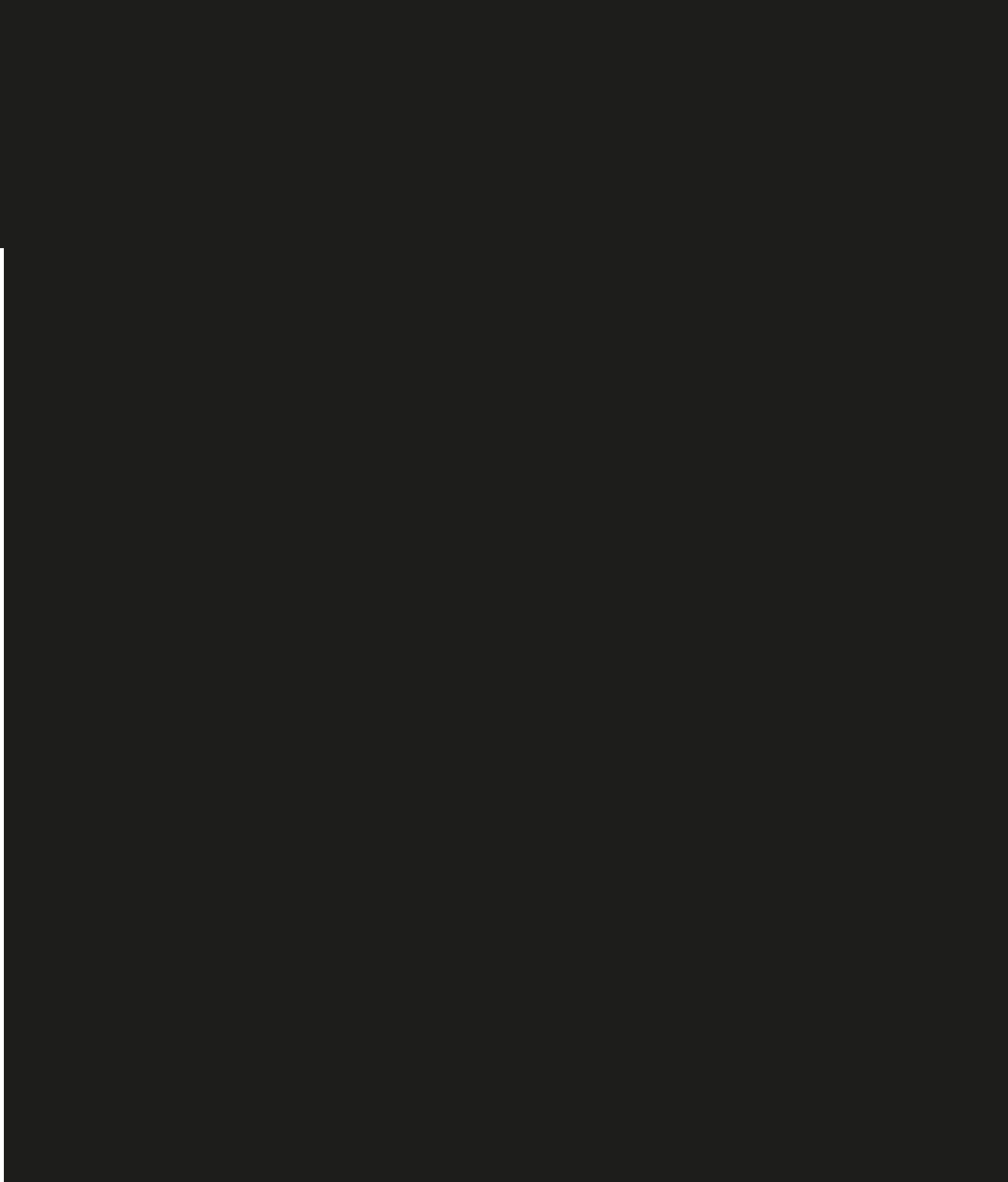




\section{Die Gestalt der Karte}

Die Karte sowie die Kartengestaltung sind wichtige Raumrepräsentationen und ein Ausdruck von epistemologischen Konzepten. Durch die Reproduktion und Verbreitung von zunächst analogen, später digitalen Karten wurde ein Zugriff auf das darin gespeicherte Wissen für eine immer breitere Bevölkerungsschicht möglich. Die Kartengestaltung als Teilgebiet der Kartenherstellung beinhaltet die Darstellung von Kartenzeichen auf der Oberfläche beziehungsweise auf einem digitalen Interface. Wissenschaftliche und technologische Errungenschaften haben sich schon immer auf die Kartengestaltung ausgewirkt. Um die Karte als Medium respektive ihren veränderten Gebrauch zu verstehen, ist daher ein Blick auf die Re-/Produktionsverfahren 
notwendig. Wie einstmals der Buchdruck revolutionierte die Software Google Earth das Handwerk der Kartografie. Neben der Vormachtstellung und dem weltweiten Einfluss des Internet-Suchdiensts Google weist dies vor allem auf einen Fakt hin: Die heutigen Kartografen sind Informatiker. Diese Tatsache hat schon jetzt einen immensen Einfluss auf die Produktion und Ästhetik von Karten. Technologische Entwicklungen wie Online-GIS und Mashup haben die Verbreitung der Karte begünstigt und eine Offnung in der Kartenherstellung ausgelöst. Heutzutage können alle Karten produzieren und online stellen und dadurch ihre subjektive Sicht auf die Welt publik machen. Kartenbasierte Dienste erlauben, bestehende Karten mit eigenen Daten zu ergänzen oder sogar komplett neue Karten zu erstellen. Dadurch verändern sich nicht nur 
traditionelle Gestaltungformen, sondern auch die Vielfalt der Darstellungen wird auf vorgegebene Normen - Templates oder Raster - reduziert. Auffallend ist, dass der Kartenstandard neuerdings durch Internetfirmen festgelegt wird. Mit der Automatisierung und Künstlicher Intel-

ligenz werden zunehmend Karten für Maschinen gestaltet; dies wird einen radikalen Umbruch für das Design von Karten bedeuten. In wenigen Jahren bereits könnten sämtliche Karten von Maschinen beziehungsweise Codes und Algorithmen produziert werden. 


\section{Analoge, digitale und interaktive Karten}

Mitte des 20. Jahrhunderts kommt es zu einer Reform in der Kartenherstellung. Der Computer wird für die Kartengestaltung verwendet, die Daten dafür werden aus einem geografischen Informationssystem bezogen. Das GIS /Vater des modernen Geoinformationssystems und Erfinder von Canada Geographic Information System ist Roger Tomlinson) ist ein Informationssystem zur Erfassung, Bearbeitung, Verwaltung und Ausgabe von räumlichen Daten. Es beinhaltet die Hard- und Software sowie die Daten und deren Anwendung. GIS-Systeme können vielseitig eingesetzt werden und sind besonders für die Kartengestaltung relevant. Wie im vorhergehenden Kapitel gezeigt, kann mit dem Geo-Web jede Information verortet und einem geografischen Informationssystem zugeordnet werden. Durch die Georeferenzierung haben die Punkte eine Beziehung zu einem Datensatz und können vielseitig genutzt werden. Durch raumbezogene Daten sind neue Dienste wie standortspezifische Applikationen entstanden. GIS-Daten waren zunächst stationär, mit Web 2.0 wurden die Daten auch mobil. Um die GIS-Dienste für private Zwecke zu nutzen, kommen tragbare Endgeräte mit Ortsbestimmung zum Einsatz. Dadurch wurde die Karte webbasiert und interaktiv und für jedermann war es fortan möglich, Karten zu bearbeiten und zu erstellen. Bekannte webbasierte Dienste sind zum Beispiel Google Maps oder die opensource basierte OpenStreetMap. Seit dem Aufkommen von mobilen GIS-Daten entwickelt sich die Karte von einer rein visuellen Oberfläche zu einem Interface, das Zugang zu einem Netzwerk ermöglicht. Mit standortbezogenen Kartendiensten wird der Zugang zum Kartenmaterial einfacher, zugleich reduzieren sich die Kosten für die Nutzer (das digitale Kartenangebot ist meist kostenlos, auf Druck und Papier kann verzichtet werden). Anfangs mit Zeichnungsprogrammen entworfen, kommen heute kartografische Spezialprogramme zum Einsatz, die auf Geoinformationssystemen und -daten basieren. Durch diese neuen Konventionen entstehen Karten getrennt vom dargestellten Raum. Ingenieur_innen, Informatiker_innen sowie die Nutzer (indem sie Daten sammeln oder Fehler melden) werden so zu Kartografen_innen. Das traditionelle Wissen zur Kartenherstellung geht verloren, respektive verändert sich. Die zuvor künstlerisch-handwerkliche Tätigkeit wandelt sich zu einer technischen Bildschirmarbeit. In Sachen ästhetischer Qualität kommen digitale Karten kaum an gestochene Karten heran. So stehen etwa die schweizerischen Landeskarten, die auf den beeindruckenden 


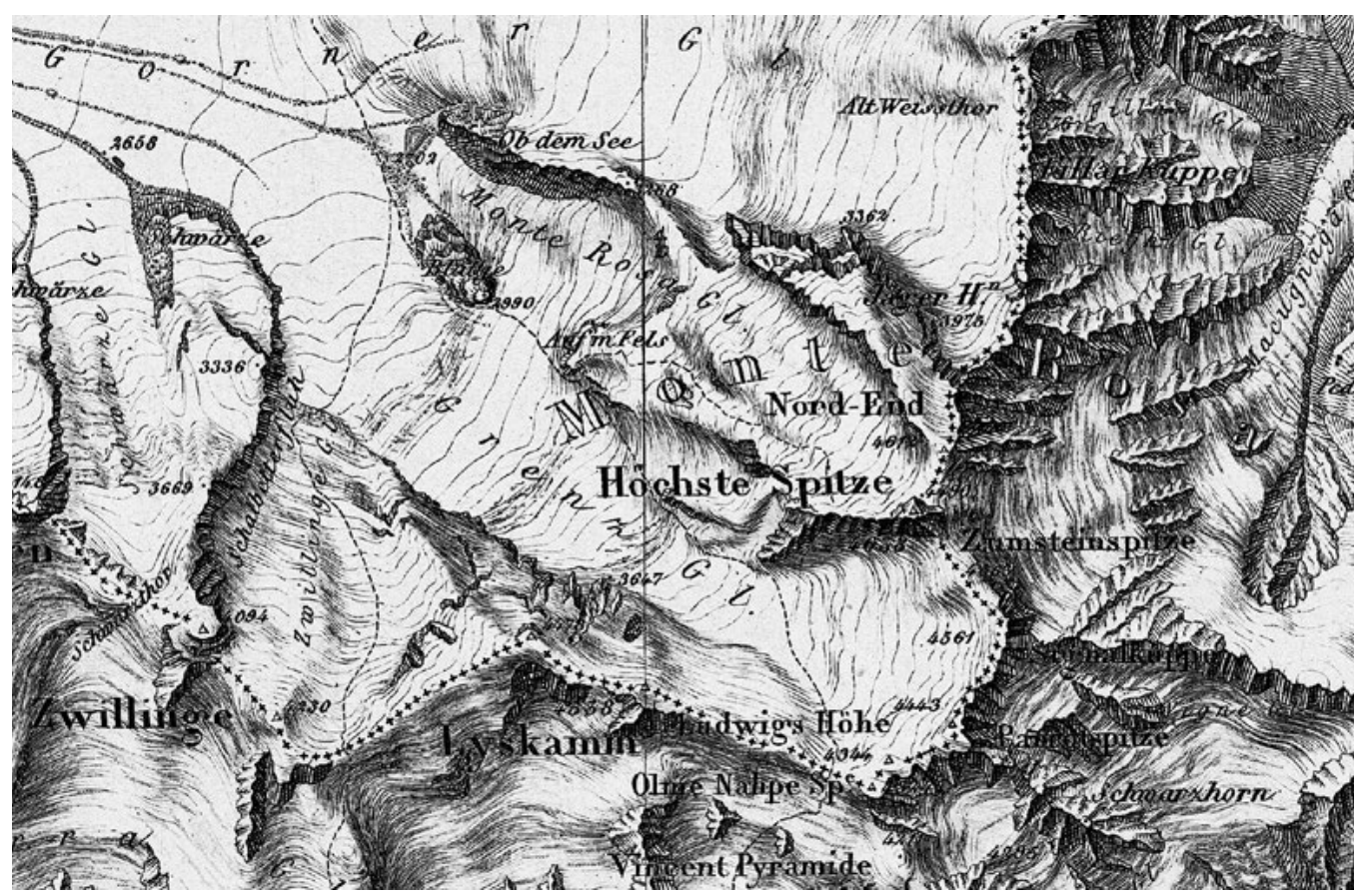

Kupferstichen der Dufourkarte basieren $^{25}$, noch heute weltweit für Präzision, Qualität und plastische Grafik. (vgl. Brändle 2014) (Abb. 18) Die Kupferstiche waren meist monochrom oder enthielten nur wenig Farbe, die dominierenden Elemente waren Linien und Schraffierungen.

Digitale Karten können in statische, dynamische oder interaktive Karten unterteilt werden. Neben einer möglichen Interaktion unterscheiden sich digitale Karten vor allem im Aufbau von analogen Karten. Analoge Karten sind immer statisch, haben einen festen Massstab und bilden einen Ausschnitt aus einem Kartenwerk ab. Digitale Karten hingegen sind in der Regel dynamisch, skalierbar (blattschnittfrei) und mit einer Plattform
Die Dufourkarte - ein nationales Symbol der Schweiz und überzeugendes grafisches Produkt. Hier ein Ausschnitt des Monte-Rosa-Massivs in den Walliser Alpen, die Höchste Spitze wurde später zu Dufour-Spitze umbenannt.

25 Vgl. swisstopo.admin.ch/de/wissenfakten/karten-und-mehr/historischekartenwerke/dufourkarte/dufourkartekulturerbe.html (abgerufen am 23.10.2018). 


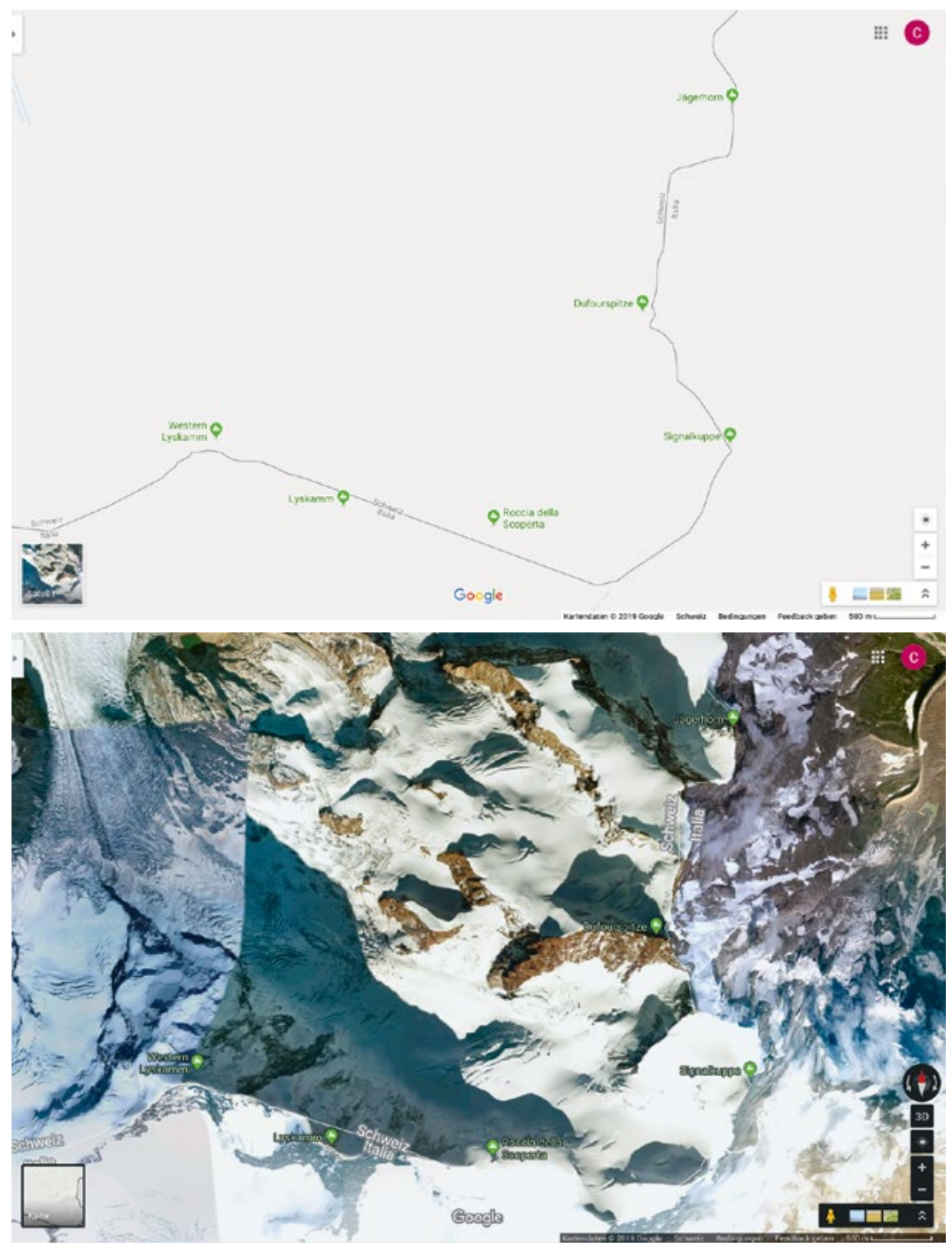

vernetzt. Durch die Skalierbarkeit entsteht bei digitalen Karten ein unendlicher Raum, der jeden Ausschnitt stufenlos vergrösser- oder verkleinerbar Die Darstellung des gleichen in Abbildung 18 gezeigten Ausschnitts des Monte-Rosa-Massivs auf Google Maps. Die Kartenansicht (oben) ist generiert und enthält kaum Informationen ausser der Landesgrenze und einigen wenigen Bergnamen. macht. Die Generierung von digitalen Anders die Satellitenaufnahme (unten), welche dagegen mit Informationen fast überfrachtet wirkt.

Karten ist technisch aufwendig, teuer und wird von Google dominiert. Google hat in wenigen Jahren - ausgehend von Google Maps (2005) - ein Kartenimperium aufgebaut und kann als Quasimonopolist ohne nennenswerte Konkurrenz bezeichnet werden. (Abb. 19) Die Online-Produkte und Kartendiens- 
te von Google sind proprietär und basieren auf einem geschlossenen Datensystem (zum Begriff von Openness siehe auch Tkacz 2015). Denn obwohl die Produkte und Kartendienste auf offenen Daten basieren, veröffentlicht Google eine gerenderte Raster-Ansicht der Karte. Raster-Ansichten sind in ein Gitter konvertiertes Raster aus Vektordaten, welche eine Nachnutzung beziehungsweise Überarbeitung unmöglich machen. Die den Karten zugrunde liegenden Geodaten werden per Lizenz von Fremdanbietern erworben und mit Satellitenbildern, Street-View-Aufnahmen und lokalen Informationen ergänzt. Da die Karten nicht mehr als Informationsspeicher dienen, kann auf Detailinformationen verzichtet werden / diese können in der dazugehörigen Datenbank gespeichert werden, vgl. Schramm 2012: 457). Die Karten sind farbiger und lassen sich je nach Modus auch plastischer und räumlicher darstellen (z.B. als Satellitenbild). Die Anbindung an eine GIS-Datenbank ermöglicht interaktive, personalisierte und sogar Echtzeit-Daten. Durch die massenhafte Verwendung von Google Maps sowie durch die Zurverfügungstellung von dessen APIs wird die Kartenästhetik zunehmend normiert und standardisiert. Google hat mit Material Design (seit 2015) gar eine eigene Designsprache entwickelt, auf welcher ihre Produkte und Dienste (insbesondere Applikationen und weniger Websites) aufbauen. Auf einer Website ${ }^{26}$ werden die gestalterischen Prinzipien und Empfehlungen veröffentlicht, auf welchen Googles Design aufbaut. Mittels Styleguide, Tutorials und frei verfügbaren Materialien wird ihre Designsprache erklärt sowie deren Verwendung und Integration auf Applikationen beschrieben. Indem APIs und gestalterische Elemente wie etwa Symbole, Stile, Typografie und Farben zur Verfügung gestellt werden, verbreitet sich das Design rasant und das Internet wird immer mehr zu einer Google-Welt. Problematisch ist dabei, dass die Webseite nicht sofort als zu Google gehörig erkannt wird.

Mit dem Aufkommen von mobilen Diensten werden vermehrt dynamische beziehungsweise interaktive Karten verwendet und produziert. Interaktive Karten sind ein konstitutives Element eines digitalen Spatial Turn, die den Raum nicht mehr als gegeben voraussetzen, sondern ständig neu produzieren. Solche Karten beinhalten Echtzeit-Daten wie beispielsweise Verkehrsströme, welche über die Karte gelegt werden (Inhalte sind voneinander separiert, ähnlich wie bei einer analogen Grundkarte). Dadurch lösen interaktive Karten die Hierarchie in der Produktion und Rezeption auf. Das Produzieren von Karten ist mit GIS-Daten für jedermann möglich und damit auch zu einem Amateur-Feld geworden. Durch das Produzieren von Karten 
durch Nicht-Kartografen wird die Welt neu vermessen, erforscht und kartiert. Die Kartierungen entsprechen kaum geografischen Abbildungen, sondern dienen dem Aufzeigen von persönlichen Zusammenhängen, Interessen und Bezügen. Dadurch, dass Google die Einbindung seiner API in fremde Applikationen und Websites ganz bewusst erlaubt, wird die Verbreitung ihrer Kartenprodukte begünstigt. Die massenhafte Verbreitung der Produkte hat zur Folge, dass Karten und Dienste überall gleich aussehen. Zugleich werden die Karten generiert, verantwortlich für die Darstellung sind Ingenieur_innen und Programmierer_innen. Benutzerfreundliche und einfach zu bedienende Interfaces verbergen die dahinterstehenden Technologien und komplexen Algorithmen und indoktrinieren im Hintergrund eine auf den ersten Blick harmlos, nett und reduziert erscheinende Welt, die Wahlmöglichkeiten jedoch von Anfang an einschränkt.

\section{Die Kartengestaltung}

Die Entscheidung, welche Informationen auf einer Karte erscheinen und welche nicht, macht diese höchst politisch. Projektionsmethode, Formgebung, Typografie und Farben können je nach gewünschter Aussage und Gewichtung angepasst werden, womit eine unterschiedliche Wirkung beziehungsweise Rhetorik erzielt werden kann. Dazu gehören auch Marginalisierung und Generalisierung. Details wie die Schraffierung von Flächen oder die Grösse von Typografie können Konsequenzen haben. So kann zum Beispiel das Weglassen eines Städtenamens den Eindruck erwecken, dass dieser Bereich nicht von Interesse ist. Siehe hierzu auch How to Lie With Maps. (Monmonier 2018) Die Kartengestaltung baut auf grafischen Grundelementen wie Punkt (z.B. für Sehenswürdigkeiten), Linie (z.B. für Strassen) und Fläche (z. B. für Landmassen) auf, die der Formenlehre (vgl. Kandinsky 1955; Klee 1979) entspringen. Auf diesen Elementen basieren auch Kartenzeichen wie etwa die Legende. Der Legende wird eine besondere politische Bedeutung beigemessen, da sie Karteninhalte verständlich und auch unverständlich machen kann. Die Vereinheitlichung von Zeichen und Symbolen - also der Versuch einer universellen Kartensprache - wird kritisch gesehen. Farbcodierungen beispielsweise basieren auf einem kulturellen Kontext beziehungsweise sind abhängig von sozio-technischen Gewohnheiten. Durch die Einführung der Lithografie wurde es möglich, die Karte in mehrere Ebenen zu separieren. Neben einer Grundkarte, die unverändert blieb, wurden weitere Ebenen angelegt, die relevante Informationen wie Flüsse, 
Strassen, Gelände beinhalteten. Durch das Prinzip des Übereinanderschichtens beziehungsweise mit der Anpassung einzelner Ebenen liess sich die Grundkarte jederzeit aktualisieren und an topografische Veränderungen anpassen, ohne dass die Karte von Grund auf neu gestaltet werden musste. Dieses Prinzip kommt auch bei der digitalen Karte zum Einsatz, die für die Inhalte auf eine GIS-Datenbank zurückgereift.

Durch die notwendige Abstraktion und Übersetzung sind Karten selektive Raumrepräsentationen. Das heisst, Karten sind eine schematische Darstellung und fussen auf einer Generalisierung sowie der Interpretation des Urhebers beziehungsweise der Urheberin. Zur Generalisierung werden gestalterische Strategien wie beispielsweise Auswahl, Verdrängung, Glättung oder Typisierung verwendet. Im Wesentlichen beinhalten die Kartenzeichen sichtbare Dinge wie Sehenswürdigkeiten, Strassen oder Flächen, die sie erkennbar wiederzugeben haben; aber auch unsichtbare Dinge wie Temperatur oder Klimazonen können auf Karten dargestellt werden. Das gestalterische Wissen wird zu Beginn des 20. Jahrhunderts für die Kartenherstellung systematisiert. (vgl.Eckert 1921) Ein frühes und wichtiges Werk hierzu ist das 1952 erschienene Buch The Look of Maps des amerikanischen Geografen Arthur H. Robinson. Das Buch, zugleich Dissertationsvorhaben des Autors, enthält Essays zur Gestalt von Karten, verzichtet jedoch bewusst auf visuelle Beispiele oder Referenzen. Aus heutiger Perspektive der Designforschung ist dies nicht wirklich schlüssig. Zu dieser Zeit gab es bereits um-

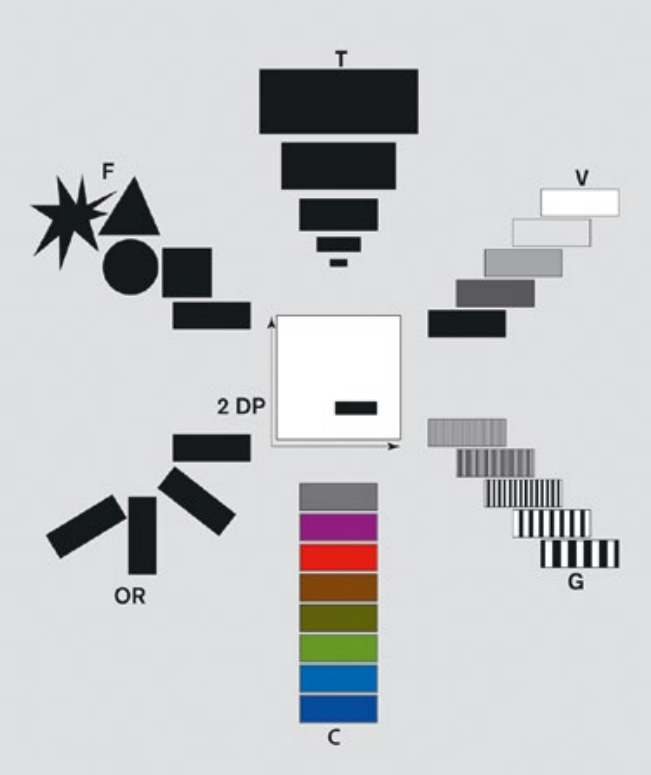

Jacques Bertin: «Semiologie Graphique» (1967), nachgezeichnet 
fassende Formexperimente und Untersuchungen zur allgemeinen Verbesserung der Lesbarkeit von Zeichen. Zum Beispiel die Studien zur standardisierten Bildsprache oder die Wiener Methode zur Bildstatistik der 1920er-Jahren des österreichischen Mathematikers Otto Neurath (1882-1945), aus welcher das noch heute verwendete ISOTYPE (International System of Typographic Picture Education, dt.: Internationales System bildhafter Erziehung) hervorgegangen ist. (vgl. Hartmann \& Bauer 2006) Mit der Einführung und Verwendung von GIS wird die Formenlehre im Hinblick auf kognitions- und wahrnehmungspsychologische Aspekte erweitert. Die Wahrnehmungspsychologie geht davon aus, dass eine Kommunikation zwischen Sender_in und Empfänger_in nur gelingen kann, wenn ein allgemein verständlicher Zeichensatz vorliegt. Die daraus hervorgegangene Semiotik (als Lehre der Zeichen, vgl. Bense 1982; Peirce 1983; Eco 1991, 2002) bildet noch heute ein tragendes Konzept für die Kartengestaltung. Mithilfe von grafischen Zeichen soll der Kommunikationsprozess unterstützt und ein allgemein verständlicher (möglichst universeller) Zeichensatz hervorgebracht werden. Die Zeichentheorie basiert auf der allgemeinen Gestalttheorie, die als grafische Semiologie bekannt ist. Das 1967 von dem französischen Kartografen Jacques Bertin (1918-2010) erschienene Buch Semiologie Graphique gilt als Standardwerk und ist zugleich ein praktischer Versuch, ein hinsichtlich Form, Farbe, Richtung, Kontrast und Muster möglichst allgemeingültiges kartografisches Zeichensystem zu definieren. (Abb. 20) Bertin erläutert verschiedene Wirkungen von Farbe, Form und Typografie und extrahiert als feste Variablen die folgenden sechs Aspekte: Grösse (T), Helligkeit (V), Textur (G), Farbe (C), Orientierung (OR) und Form (F) (für weitere Standardwerke zur Kartengestaltung siehe z.B. MacEachren 1995; Krygier \& Wood 2005 oder Brus/ Vondrakova/Vozenilek 2015).

Eine Schwierigkeit bei der Kartengestaltung ist die Unmöglichkeit, die dreidimensionale Erdkugel zweidimensional mathematisch korrekt (und ohne Verzerrungen) abzubilden. Durch die Erdkrümmung können Karten entweder winkel- oder flächentreu sein, beides zugleich ist nicht möglich. Die Erstellung der ersten winkeltreuen und auch weitgehend formentreuen Karte gelang 1569 dem Kartografen Gerhard Mercator (1512-1594). (Abb. 21) Auf der Karte befindet sich Europa im Mittelpunkt und wirkt stark vergrössert. Mit einer Projektionsmethode, bei der Flächen desto grösser dargestellt werden, je weiter sie vom Äquator entfernt liegen. Dies hat den Effekt, dass Afrika und Südamerika im Verhältnis zu ihrer Grösse als viel zu klein erscheinen und Australien kleiner als Grönland dargestellt wird, obwohl es in Fakt etwa dreimal so gross ist. Die Mercator-Karte gilt 


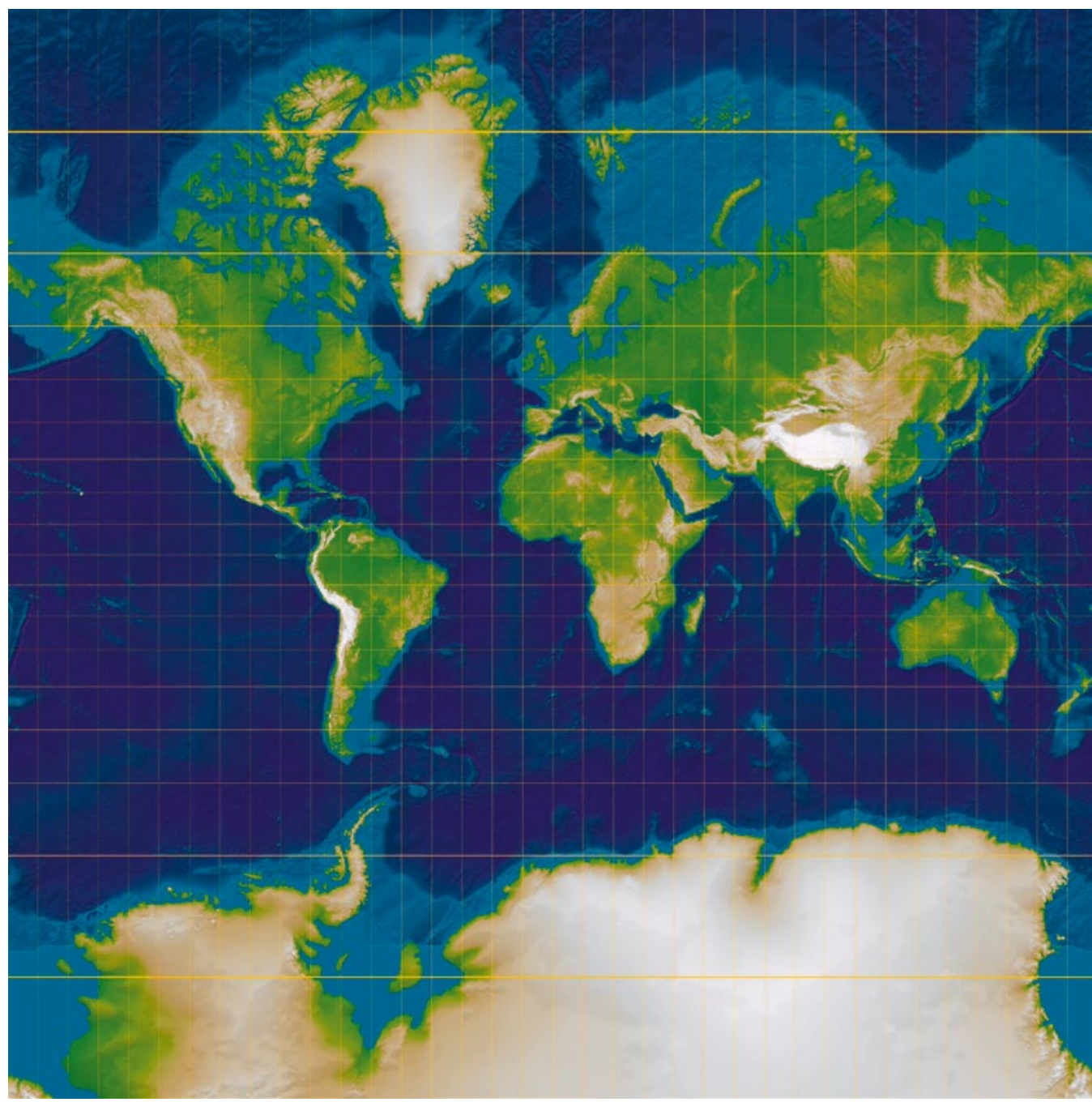

bis heute als Standard, auch wenn die Nordausrichtung sowie eine eurozentrische Weltsicht entlang der kritischen Kartografie und im Kontext des Postkolonialismus seit dem 20. Jahrhundert kontrovers diskutiert werden. 1974 stellte der deutsche Historiker und Kartograf Arno Peters (2016-2002) die bis dahin geltende Weltsicht radikal infrage, indem er für die Weltdarstellung auf eine flächentreue Perspektive zurückgriff. (Abb. 22) Bei dieser Darstellungsform werden Landmassen desto grösser dargestellt, je näher sie am Äquator
Die der Mercator-Karte zugrunde liegende winkeltreue Projektion ist die weltweit am häufigsten verwendete Projektionsmethode. 


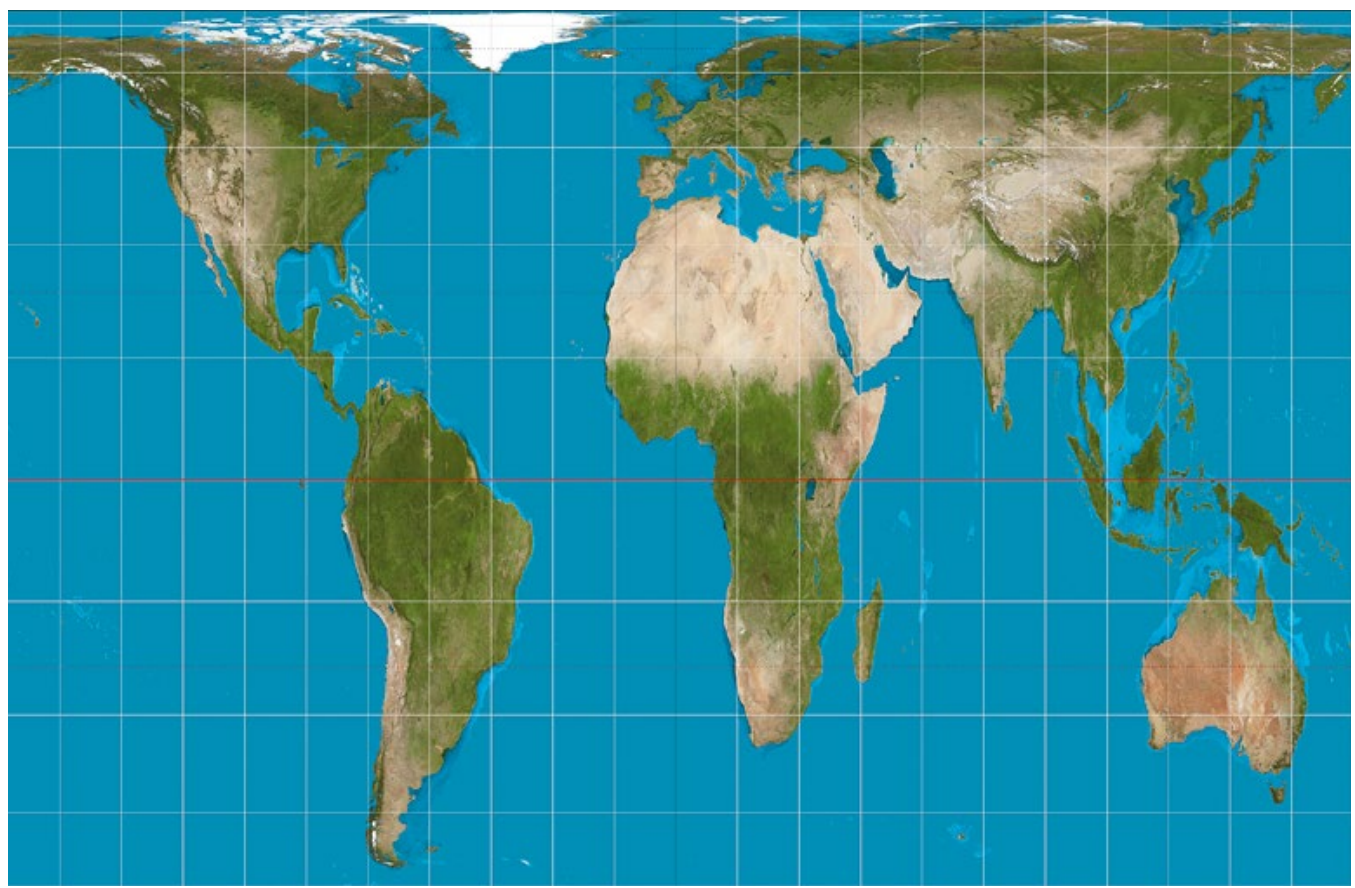

liegen. Dadurch nehmen die einzelnen Kontinente teilweise eine ungewöhnDie Peters-Projektion von 1974 gilt als Gegenliche Form an. Die Peters-Projektion, die die exakten Landmassen abbildet, gilt bis heute als Gegenentwurf zu der gängigen Mercator-Karte, der, wie bereits erwähnt, ein eurozentristischer Blick zugrunde liegt. Peters äusserst sich zur Mercator-Projektion wie folgt: "[I]t over values the white man and distorts the picture of the world to the advantage of the colonial masters of the time." (Crampton 2010: 92) Auch Google Maps gründet auf einer WebMercator-Projektion, der seit der Neuzeit favorisierten Projektionsmethode. «Die Web-Mercator-Projektion hat neben einigen technischen Vorteilen die Eigenschaft, dass sie bei jeder Bildverschiebung und bei jedem Zoom in jeder Region, an jedem Ort und in jedem Zoomfaktor anwendbar ist.” (Stirnemann 2018: 155) Durch den ubiquitären Gebrauch von Google Maps 
zeigt sich - trotz Bemühungen der kritischen Kartografie - weiterhin eine Weltanschauung, in welcher Europa und Amerika im Zentrum stehen. Dass die Kartengestaltung auch politisch ist, wird in der Entscheidung der Google-Ingenieure für die Verwendung der Mercator-Projektion deutlich, die Google ungeachtet der seit einigen Jahren geäusserten Kritik für seine Kartendienste verwendet. Seit 2018 hat Google die Projektionsform dahingehend angepasst, dass die Mercator-Ansicht bei einer geringen Zoomstufe automatisch auf eine Globusansicht wechselt, wodurch die Grössenverhältnisse der Land- und Wassermassen stimmen.

Die Vorstellung, dass Karten ein objektives und wissenschaftliches Abbild der Welt sind, ist nach wie vor ein weit verbreiteter Mythos. Auch auf generierten Karten - von denen viele von einer vermeintlichen Realität ausgehen - sind Projektionsprobleme ungelöst, dazu kommen geopolitische Schwierigkeiten und Interessenskonflikte sowie Marginalisierungen. Trotz technischen Fortschritts gibt es bisher keine korrekte beziehungsweise umfassende Karte. Fotorealistische Abbildungen der Welt (wie Satellitenbilder) werden im allgemeinen Gebrauch nicht als Karten verstanden, da sie zu viele überflüssige Details enthalten, um sich damit zu orientieren oder navigieren zu können.

\section{Bezug zur Perspektive}

Für die möglichst getreue Wiedergabe der Erdoberfläche waren, wie bereits erwähnt, die perspektivische Darstellung räumlicher Verhältnisse und die daraus hervorgegangenen Projektionsmethoden ein wichtiger konzeptioneller Schritt. Einen wichtigen Beitrag hierfür leistete die Entdeckung (der Gesetze) der Zentralperspektive durch florentinische Architekten im 15. Jahrhundert. Diese ermöglichte eine räumliche Raumpräsentation anstatt der bisher flachen Ansicht. Daraus (siehe auch Panofsky 1980) entwickelten sich die Projektionsmethoden, die auch Einfluss auf die Kartengestaltung hatten. Nachdem in der Frührenaissance die geometrischen Regeln der Perspektive bekannt geworden waren, begannen Künstler, aus der Vogelperspektive Panoramen von Städten zu zeichnen. Ein dreidimensionaler Raum in eine zweidimensionale Form zu übersetzen, ist, wie weiter vorne beschrieben, eine Herausforderung. Entsprechend anspruchsvoll war die Zeichnung solcher Panoramen ohne jegliche technischen Hilfsmittel. Allerdings dienten die Stadtansichten auch nicht der Orientierung, sondern waren als Wandschmuck für bürger- 


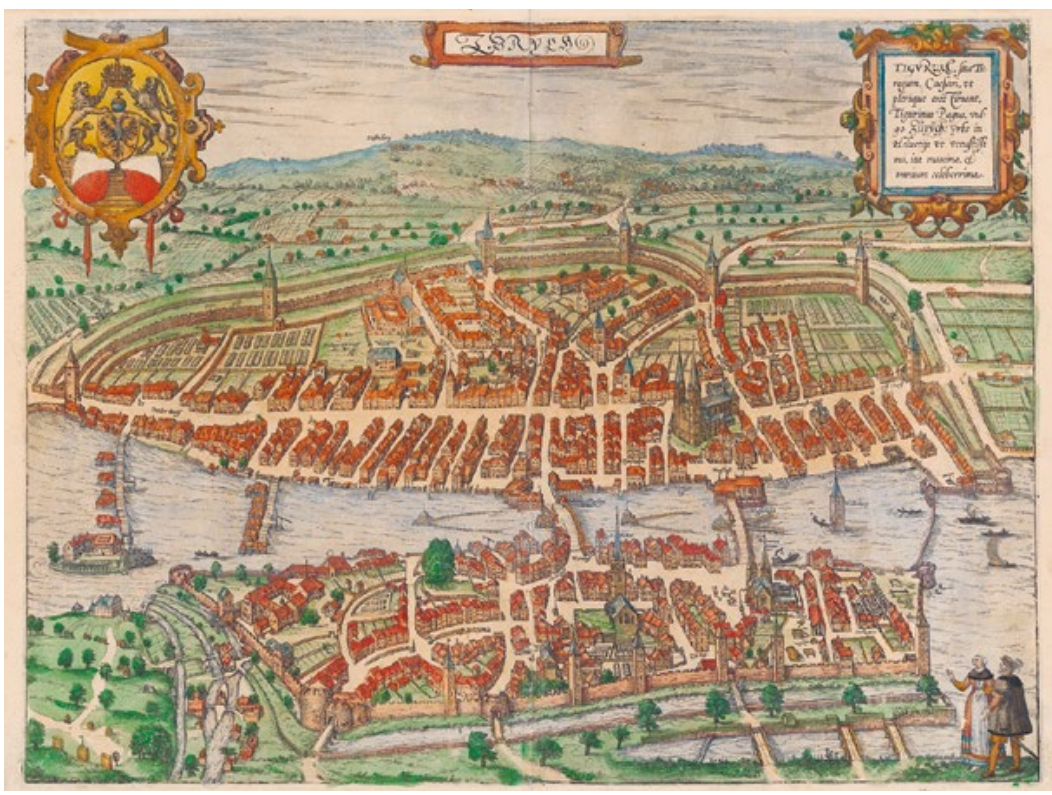

liche Haushalte gedacht. Diese Stadtansichten in Karten zu übersetzen, war Stadtansicht von Zürich (um 1581) aus der Civitates Orbis Terrarum (umfasst über 600 Stadtansichten ein enormer konzeptioneller Schritt, wie der Grafiker Edward Tufte ausführt: "Trotz quantifizierbarem Massstab und Raster gleichen die Panoramen mehr Miniaturdarstellungen. Denn um eine Beziehung zwischen Grössenverhältnissen darstellen zu können, müssen die räumlichen Massstäbe durch abstrakte Massstäbe ersetzt werden, die nicht auf geografischen Analogien basieren.» (Tufte 1997: 14, Übersetzung der Autorin) Mit anderen Worten: Es müssen die relevanten Informationen ausgewählt und die darzustellenden Informationen in ein grafisches Zeichensystem übersetzt werden, damit sie wiedererkannt werden. Der erste Stadtatlas (Civitates Orbis Terrarum) wurde 1572 von Georg Braun und Franz Hogenberg in Köln veröffentlicht. (Abb. 23) 1618 umfasste dieser Atlas bereits sechs Teile mit 363 Stadtkarten von Köln bis Paris und Rom, von Peking bis Goa und Mombasa. Jede die- 
ser Stadtkarten zeigt eine räumliche Darstellung der topografischen Gegebenheiten wie das Strassennetz mit einzelnen Gebäuden, Schiffen, Kanälen, Häfen oder anderen Landmarken. Zudem waren die Ansichten mit Erläuterungen zur Geschichte, zur Lage und zur wirtschaftlichen Bedeutung der Stadt versehen.

Mit der Verbreitung der Lithografie im ausgehenden 19. Jahrhundert wurde die Panoramakarte zum Massenphänomen. Dieser Kartentyp wurde zum Vorläufer der modernen Reisekarte. Ausgehend von der Panoramakarte und der dort eingesetzten Vogelperspektive fand die Parallelperspektive Eingang in die Kartengestaltung. Die Parallelperspektive, genauer die axonometrische Projektion, zeigt alle drei Dimensionen - Grundriss, Schnitt und Ansicht von Objekten gleichzeitig und unterstützt dadurch das räumliche Vorstellungsvermögen. Eine frühe Form der axonometrischen Projektion, ist der "Plan de Paris" aus dem Jahre 1739 von Louis Bretez und MichelÉtienne Turgot. In den 1960er-Jahren wurde die axonometrische Projektion durch den Grafiker Hermann Bollmann (1911 - 1971) massgeblich weiterentwickelt. Für Midtown Manhatten in New York City zeichnete er 1962 eine touristische Karte von hoher ästhetischer Qualität. (Abb.24) Als Vorlage für seine handgezeichnete Karte hat Bollmann mehrere Tausend Luft- und Bodenaufnahmen verwendet haben. Die Parallelperspektive wird seitdem gelegentlich verwendet, als Standard durchsetzen konnte sie sich jedoch nie. Und dies obwohl seit der Kognitionspsychologie bekannt ist, dass das menschliche Auge auf Strassenhöhe mit flächigen Projektionen wenig anzufangen weiss ( $z u$ abstrakt ist die Information). Der Abgleich der Karte mit der räumlichen Umgebung wird zu anspruchsvoll. Nicht zuletzt deshalb, da die Karte eine Abstraktion ist, ein Hilfsmittel zur Orientierung, und mit der physischen Umgebung wenig zu tun hat. Der Abgleich wird zusätzlich erschwert, da das Gehirn bei der Projektion aus einer Fussgängerperspektive Probleme hat, die abstrakten Grundrisse in dreidimensionale Bauten zu übersetzen. Kognitive Untersuchungen zeigen, dass Darstellungen in axonometrischer Projektion einen einfacheren Zugang ermöglichen. Es stellt sich die Frage, weshalb sich die Parallelperspektive trotzdem nicht durchsetzen konnte. Meine Vermutung ist, dass das Verfahren grosse Expertise und zeichnerisches Talent erfordert und wesentlich aufwendiger als der Entwurf eines flächigen Grundrisses ist.

Seit dem technologischen Wandel sind mit dem Computer generierte Karten üblich geworden. Dahinterstehen Programmier_innen und Ingenieur_innen, die die Karten generieren. Oftmals mangelt es jedoch den Nutzern an gestalterischem Grundwissen, das zum intuitiven Verständnis einer Karte beitragen kann. Google Maps tätigt 

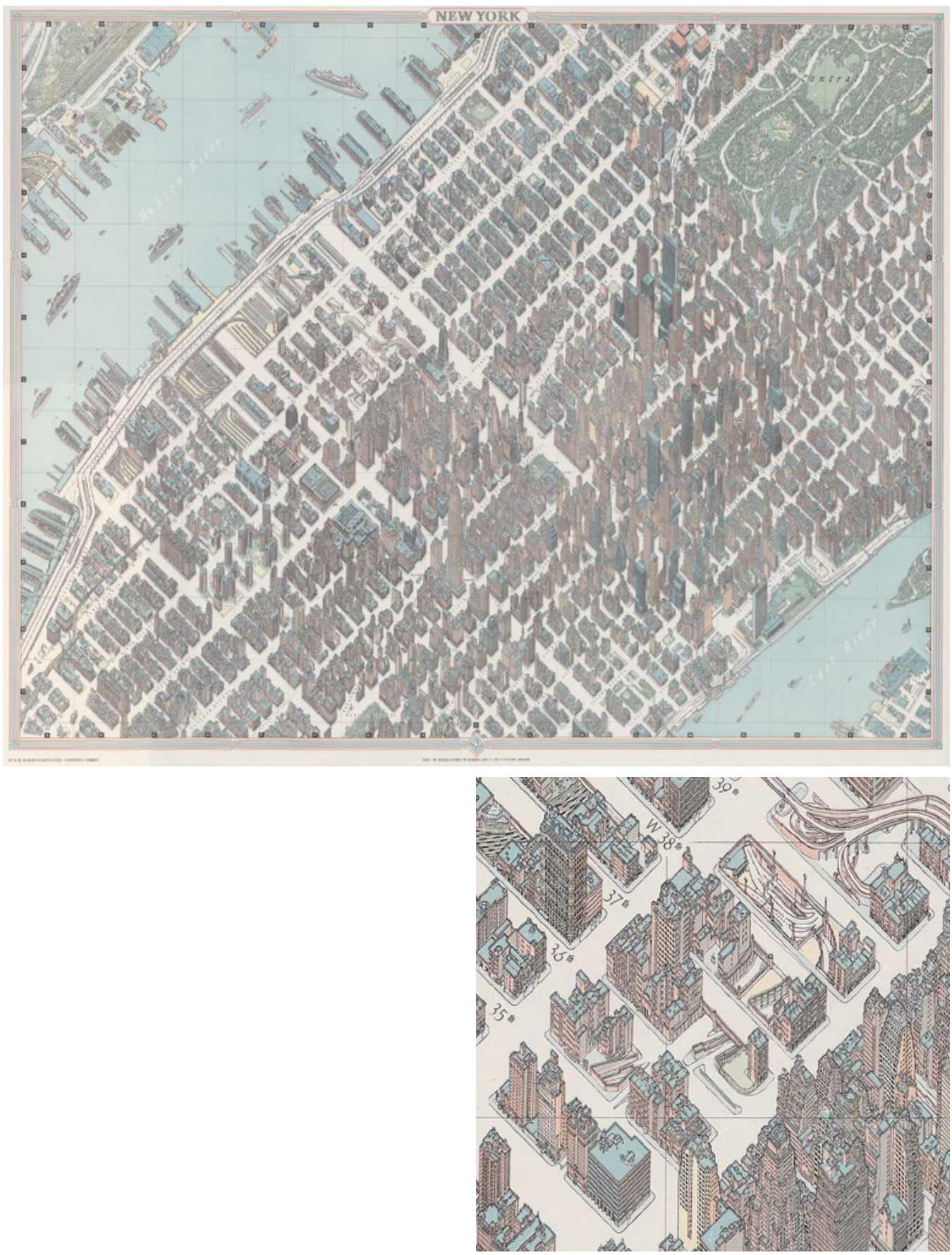

Hermann Bollmann: Karte von Midtown Manhatten (1962) (oben). Bei der verwendeten Parallelperspektive werden die Objekte räumlich auf einer Ebene dargestellt und vermitteln so einen Eindruck von Tiefe und Räumlichkeit (siehe Ausschnitt unten). 
deshalb erhebliche Investitionen, um Navigationselemente und Werkzeugfunktionen entsprechend zu verbessern. Wesentliche Verbesserungen können in dreidimensionalen Ansichten, Bildüberlagerungen, Überflugmodi sowie Standortsuche ausgemacht werden. Weitere hilfreiche Funktionen sind beispielsweise Zoom, Ortseingabe, persönliche Markierungen und Scroll-Möglichkeit. Mit den erwähnten Eigenschaften, die das Unternehmen in seine OnlineKarten integriert, wird in Sachen Ästhetik mit tradierten Konventionen analoger Karten gebrochen. Auf Google Earth oder bei Google Maps lässt sich beispielsweise der Modus von einer zweidimensionalen Kartenansicht in eine dreidimensionale Satellitenaufnahme oder auch auf eine Strassenperspektive wechseln. So hat der Nutzer jeweils die Möglichkeit, zwischen einer Plan- oder einer Satellitendarstellung zu wählen und hin und her zu wechseln. Zugleich kann wahlweise auf Augenhöhe durch die Strassen navigiert oder aus der Vogelperspektive auf das Geschehen heruntergeschaut werden.

Die technischen Fortschritte bringen allerdings nicht nur Vorteile, sondern machen auch anfällig für mögliche Fehler. Neben veraltetem Kartenmaterial und falschen Inhalten kann es aufgrund verschiedener Plattformen zu systemtechnischen Problemen kommen. Die Bilder für Google Earth basieren einerseits auf einem topografischen Modell, andererseits auf Satellitenaufnahmen. Die Kombination der verschiedenen Systeme kann zu eigenartigen Fehldarstellungen führen - ähnlich wie digitale Artefakte. Bei Google ist zwar eine grosse Anzahl an Personen damit beschäftigt, solche Fehler zu suchen und zu beheben, trotzdem finden sich immer wieder fehlerhafte Darstellungen. Der Künstler Clement Valla thematisiert mit "Postcards from Google Earth" diese Bruchstellen. (Abb. 25) Seine Postkarten sind Screenshots aus dem Internet und Momentaufnahmen von Störungen und Fehlberechnungen der Google-Earth-Software (Fehler diesbezüglich, wie die Plattformen die Welt automatisch rendern), bei denen Strukturen, die nicht zusammengehören, falsch gerendert und zusammengeschoben werden. Valla führt dazu allgemeiner aus: “We have to relearn how to read these hybrids. Are they flat maps? Spatial images? 3-D objects? 2-D images? "27 In der Tat ist eine Zuordnung solcher computergenerierten Bildern schwierig geworden. Als Karten sind sie oftmals zu wenig abstrakt und als Bild wirken sie nicht authentisch.

27 www.theguardian.com/artanddesign/shortcuts/2016/may/02/postcardsgoogle-earth-artist-clement-valla (abgerufen am 13.05.2019). 
Abb. 25

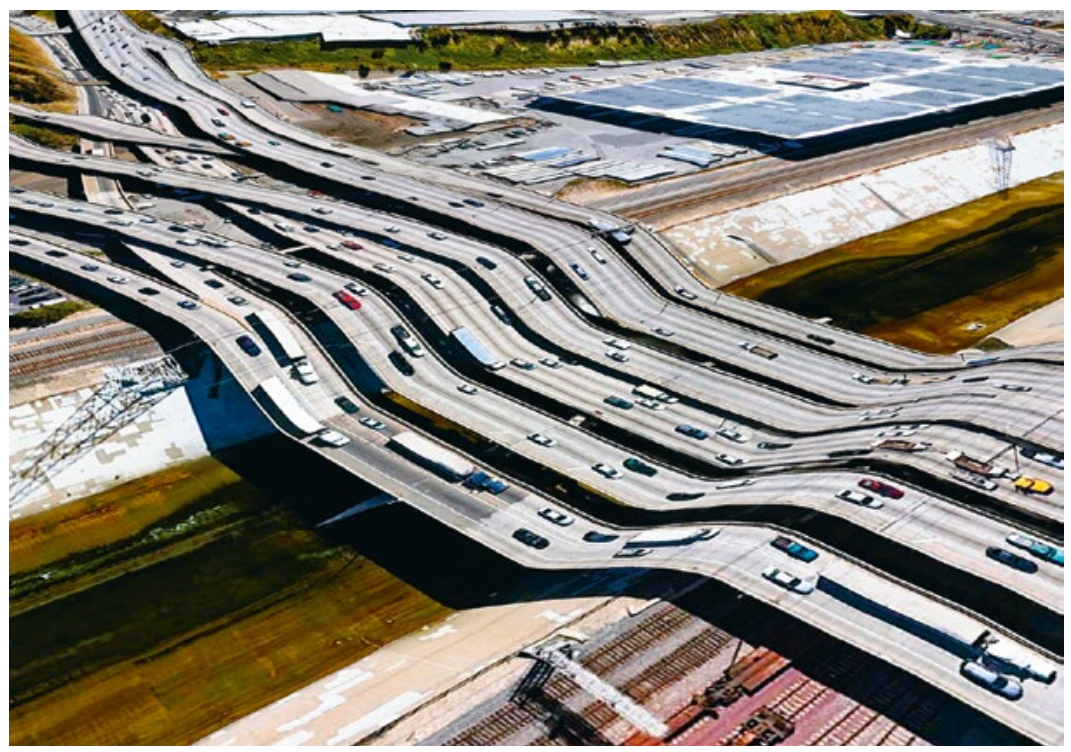

Clement Valla: «Postcards from Google Earth» (2019) 


\section{Von Human-centered Design zum Design für Maschinen}

Durch den technologischen Fortschritt verändern sich nicht nur Form, Gestalt und Inhalt von Karten, sondern auch, an wen die Karte adressiert wird und von wem die Karte gestaltet ist. Einerseits werden Karten nicht mehr ausschliesslich von Menschen, andererseits auch nicht mehr nur für Menschen gestaltet. Einer der Vordenker von maschinengeneriertem Design und der Gestaltung mit Algorithmen ist der japanische Designer John Maeda. Bereits in den 1990er-Jahren experimentierte er mit Design by Numbers an einem durch den Computer generiertem Grafik-Design. Zu dieser Zeit - in den Anfängen eines mit Computer gestalteten Entwurfs - war ein Design durch ein autonomes System noch unvorstellbar. Mit neu entstandenen Technologien wie Künstliche Intelligenz, Algorithmen und Bots wird dieser Gedanke jedoch greifbarer. Ein Pionier in Sachen Daten, Visualisierung und Code ist der italienische Architekt und Ingenieur Carlo Ratti, der das MIT Senseable City Lab in Massachusetts leitet. Seine wegweisenden Arbeiten werden weltweit ausgestellt und publiziert (vgl. etwa Offenhuber \& Ratti 2013). Mit seiner Forschungsgruppe untersucht er den Einfluss neuer Technologien auf Städte und wie diese dadurch gestaltet werden können.

In Zukunft wird anstelle für Menschen wohl vermehrt für Maschinen gestaltet werden, zum Beispiel bei selbstfahrenden Autos. Dadurch verlagert sich der bisher gängige Begriff von menschenzentrierter Gestaltung (Human-centered Design) zu einem Design für Maschinen. Der US-amerikanische Designtheoretiker Benjamin Bratton spricht bereits von einem Machine User (respektive Animal User und AI-User) und plädiert, den Designbegriff radikal weiterzudenken: «[T] $\mathrm{O}$ design for what comes next, what comes outside, what has already arrived for the synthetic User-subjects for which another geopolitics is derived.” (Bratton 2015: 289) Der Künstler James Bridle bringt Zukunftstechnologien wie Maschinelles Sehen oder Künstliche Intelligenz mit sozialen Themen wie Automatisierung oder den Arbeitswandel (Industrie 4.0) zusammen und hinterfragt die Macht der dahinterstehenden Internetunternehmen. Der Künstler thematisiert unter anderem Fragen nach ethischer und sozialer Gerechtigkeit sowie das blinde Vertrauen der Menschen in Maschinen und Ingenieursleistungen (siehe hierzu auch seine Publikation The New Darke Age, 2018). In Autonomous Trap 001 (2017) beschäftigt sich der Künstler mit der Technologie und dem Funktionieren von selbstfahrenden Autos. Bridle stellt zum Beispiel einem autonomen Fahr- 


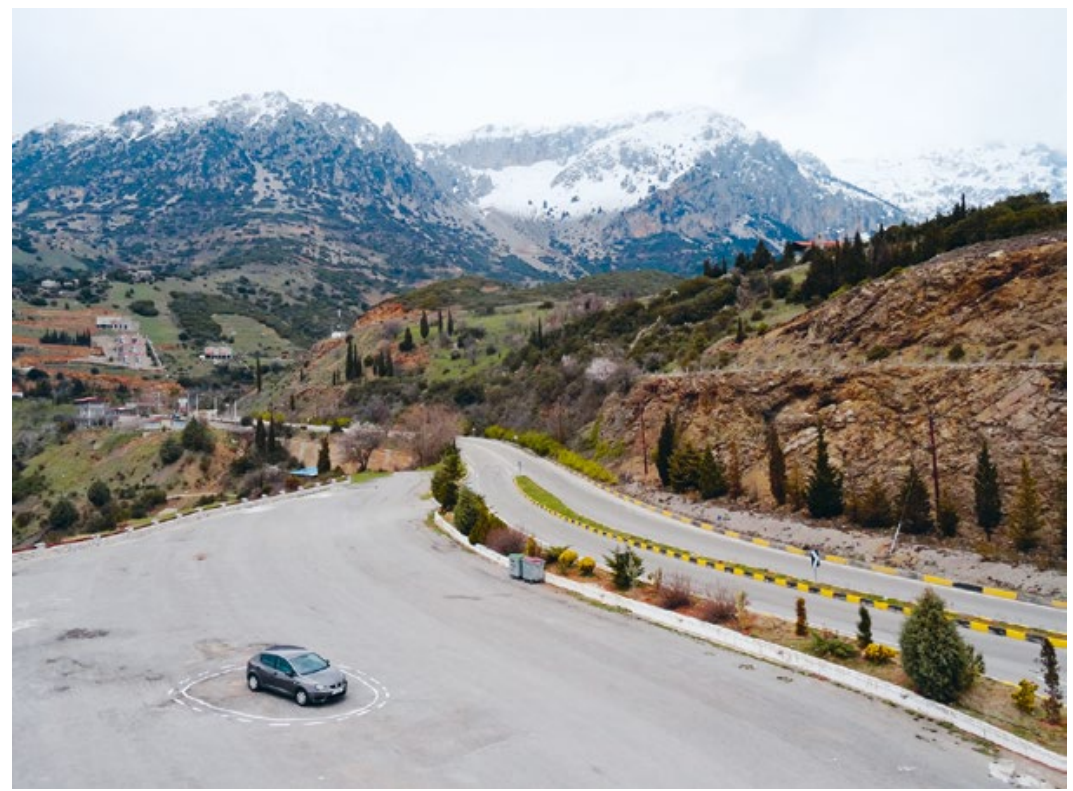

zeug eine Falle - ein aus Salz gezeich- James Bridle: «Autonomous Trap 001» (2017) neter Kreis auf einem Parkplatz, der aus einer aussen gestrichelten und innen durchgezogenen Linie besteht. (Abb. 26) Die Linien bringen den Bordcomputer dermassen durcheinander, dass das Auto nicht mehr weiterfahren kann. Die gestrichelte Linie erlaubt zwar das Hineinfahren in den Kreis, die durchgehende Linie hindert das Auto jedoch daran, den Kreis wieder zu verlassen. Diese Arbeit zeigt auf humoristischabsurde Weise die blinden Flecken neuer Technologien auf.

Um räumliche Entwurfspraktiken weiterzudenken, ist es daher wichtig, sich zunächst bewusst zu werden, dass Maschinen und Menschen Räume unterschiedlich erfassen und wahrnehmen. Shannon Mattern, die zu Beziehungen zwischen Form und Materialität von Medien und Räumen forscht, bezieht sich in ihrem vielbeachteten Aufsatz «Mapping's Intelligent Agents " 
$(2017)^{28}$ zur Operationalisierung von Raum durch Maschinen auf maschinelle Kartierungsagenten wie Sensoren, Drohnen und Roboter. Mit optischen Messverfahren wie Lidar (Light detection and ranging) ist es möglich geworden, einen hochauflösenden 3-D-Scan von Objekten zu erstellen. Obwohl der Scan viel mehr Informationen als ein fotografiertes oder gemaltes Bild aufweist, bleibt es eine Abstraktion. Das heisst, ein von Menschen unterstützter beziehungsweise konzipierter Entwurf kommt viel näher an die physische Realität heran als ein durch eine Maschine generiertes Bild. Über Sensoren (etwa akustische und optische Messverfahren wie Sodar und Lidar) vermessen selbstfahrende Autos die Umgebung und generieren ein Bild, auf welches der Bordcomputer reagiert. Für die Steuerung des Fahrzeuges ist eine generierte und individuelle Echtzeitkarte der Umgebung (3-D-Coderaum) verantwortlich, wie Mattern ausführt: “Das Auto (sieht, und hört, die Welt als einen organisierten dreidimensionalen Coderaum aus Zeichen und Linien, der den Betrieb steuert und gleichzeitig eine Ansammlung reflektierender Objekte enthält (Fussgänger, Fahrräder, Autos, Leitlinien, Steine und Bäume). Die reflektierenden Objekte können die Ordnung des Coderaums unterbrechen und müssen deshalb ihre Beziehung zu jedem Auto neu verhandeln. " (Mattern 2017, Übersetzung der Autorin) Da Maschinen die Umgebung anders lesen und verarbeiten, wird eine visuell verständliche Abstraktion der Welt, wie dies bisher bei für Menschen gestalteten Karten gebräuchlich war, nicht mehr nötig sein. Die Technologie basiert auf selbständigem Erfassen und Interpretation der Daten. Bei einer hohen Datenmenge werden maschinelle Lernsysteme eingesetzt, um die Daten zu priorisieren. Wie Maschinen Raum konzipieren und operationalisieren wird auch einen Einfluss auf das räumliche Denken von Menschen haben. Für Maschinen als Verarbeiter von Karten ( zu maschinellem Sehen siehe auch Graham 2005) werden künftig ganz andere Kriterien wichtig sein, wie primär visuelle Standards für den Menschen. (Abb. 27)

28 Siehe auch hier: www.placesjournal.org/article/mappings-intelligentagents/?cn-reloaded=1 (abgerufen am 20.02.2018). 


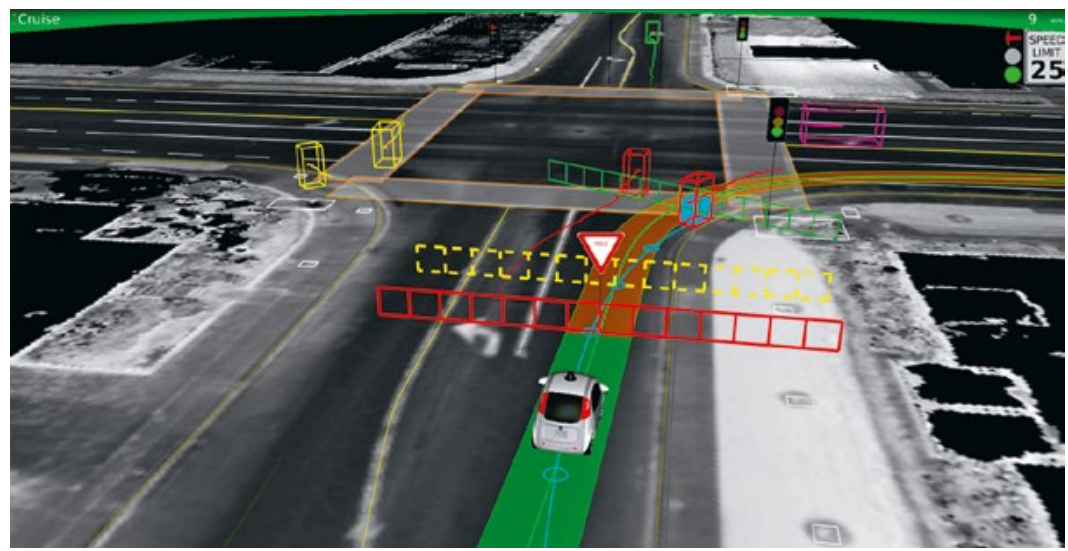

Im Bild dargestellt ist der 3-D-Coderaum eines selbstfahrenden Fahrzeugs von Waymo.

Das Auto gewährt einem entgegenkommenden Radfahrer Vortritt (rotes Rechteck), obwohl dieser auf der falschen Spur fährt. 


\section{Die Benutzerschnittstelle zwischen Mensch - Computer (oder System)}

Wie im Kapitel Raum - der digitale Spatial Turn erörtert, kann die Karte als konstituierendes Element eines digitalen Spatial Turn herangezogen werden. Die Gründe für den Paradigmenwechsel sind einerseits im alltäglichen Gebrauch von Geomedien, andererseits in der dadurch begünstigten Möglichkeit zur Produktion, Visualisierung und Distribution von Geodaten zu suchen. Durch die immense Menge an georeferenzierten Daten und Informationen wandelt sich die Karte von einem Orientierungsund Navigationshilfsmittel zu einer Suchoberfläche und zu einem räumlichen Netzwerk. Sie schafft eine Schnittstelle zwischen Mensch, Maschine und Raum beziehungsweise Environment und erzeugt 
dadurch eine physische Interaktion. Unter Interface wird begriffsgeschichtlich die Benutzerschnittstelle zwischen Mensch-Maschine (Hard- oder Software) verstanden. Da aus heutiger Sicht nicht mehr wirklich unterscheidbar ist, ob es sich um eine Maschine oder einen Computer handelt, wird hier von Computer (oder System) gesprochen.

Interfaces sind allgegenwärtig und werden immer unsichtbarer (Ubiquitous Computing). Mit der Nutzung des Computers als Alltagsgerät wird es deshalb notwendig, ein verständliches Benutzungssystem für Nicht-Spezialist_innen zu schaffen. Dazu bedarf es einer Schnittstelle für den Zugang zu den Daten sowie für die Darstellung und deren Bearbeitung. Durch die Notwendigkeit einer verständlichen Benutzeroberfläche wurde das Interface zum Gegen- 
stand von Design und die Gestaltung der Schnittstelle zur Disziplin von Interaktionsdesign. Das Interfacedesign beinhaltet die Schnittstelle $\mathrm{zu}$ digitalen Informationen wie Fenster, Icon oder Menü sowie Steuerelemente wie Maus oder Tastatur. Bedingt durch die technologischen Entwicklungen kommen vermehrt Systeme zum Einsatz, die anders gesteuert werden und andere Sinne ansprechen, wie zum Beispiel Touchscreen oder Spracheingaben wie bei Alexa und Siri. Im Interaktionsdesign wird die Benutzerfreundlichkeit sowie die Ergonomie der Schnittstelle gestaltet. Dabei sind vor allem drei Aspekte zentral: die Ein- und Ausgabe, die Interaktion sowie die Darstellung von Daten und Information. Interaktionsdesign beschäftigt sich demnach mit dem einfachen Auffinden von Inhalten und der Navigation durch die Informationen. 


\section{Die grafische Benutzeroberfläche}

Ein Computer wird meistens mit Eingabegeräten wie Maus oder Tastatur gesteuert. Der Dialog zwischen Mensch und Computer beziehungsweise dem Display (bildschirmbasierte Medien) setzt daher ein interaktives System (Steuerung von Verhalten und Aufgabe) mit einem Eingabegeräte voraus. Der Lichtgriffel (Sketchpad) von Ivan Sutherland schaffte in den 1960er-Jahren erstmals eine dialogfähige Schnittstelle zwischen Mensch-Computer. Im gleichen Zeitraum erfand Douglas Engelbart (1925-2013) ein Zeigegerät (Pointing Device) und damit die Möglichkeit, abstrakte Zeichen auf einem Bildschirm zu steuern. Nach der erfolgreichen Gestaltung dieser Ein- und Ausgabegeräte konzentrierten sich die Entwicklungen auf die grafische Oberfläche - auf den Bildschirm. Dem US-amerikanischen Informatiker Alan Kay vom Palo Alto Research Center (PARC) gelang in den 1970er-Jahren mit der Gestaltung von grafischen Zeichen (Graphic Icons) ein wegweisender Entwicklungsschritt. Die grafische Benutzeroberfläche löste die bis dahin übliche kommandozeilenbasierte Oberfläche (command Line) ab. Dadurch wurde die Bedienung eines Computers auch für Nicht-Programmier_innen verständlich und möglich. Die damaligen innovativen Steuerungselemente wie Fenster, Maus, Symbole und Bedienelemente zur Eingabe und Bearbeitung von Daten entsprechen weitgehend noch dem heutigen Computerstandard. Die grafische Benutzeroberfläche/Graphical User Interface) beinhaltet Fenster zur Organisation, Symbole für deren Zugang sowie Bedienelemente wie Menü und Zeigegerät (WIMP - Window, Icon, Menu und Pointing Device).

Durch die Einführung von Symbolen und sich überlappenden Fenstern gelang die erste grafische Benutzeroberfläche, die den Durchbruch für ein verständliches Betriebssystem ebnete. Zum intuitiven Verständnis der komplexen Zusammenhänge wird die bis heute übliche Schreibtischmetapher (Papierkorb, Ordner, Dokumente) entwickelt und mit Apple Lisa im Jahre 1983 eingeführt. Mit Apple Macintosh gelang Steve Job 1984 eines der ersten marktfähigen Betriebssysteme, auf welchem noch heute der Standard aufbaut. Der erste Macintosh-Computer von 1984 verzeichnete bereits eine grosse Auswahl an Schriften, was einen erheblichen Fortschritt in Sachen Lesbarkeit darstellte. Der Font Chicago aus diesen Anfangszeiten dürfte einigen noch bekannt sein. Job entwickelte eine grafische Benutzeroberfläche in ästhetisch-ansprechendem und nach heutigem Verständnis nutzerzentriertem Design. Damit legte er den Grundstein für den Durchbruch des Computers als Alltagsgerät und setzte erste Parameter für moderne Interaktionskonzepte. Neben den Ein- 
gabe- und Steuerungselementen, der Schreibtischmetapher sowie der Typografie waren die Farbgestaltung und das (bewegte) Bild weitere Schlüsselelemente. Mit der Gestaltung von Interfaces für Maschinen kommt es zu einem Paradigmenwechsel von einem nutzerzentrierten zu einem maschinenzentriertem Design. Wie sich dies auf die Gestaltung auswirken wird, ist weitgehend unerforscht.

\section{Natural User Interface - akustische, haptische und muskuläre Signale}

Mit der Ubiquität von Computern und dem Internet der Dinge werden neue Formen von Benutzungsschnittstellen bedeutsam. Neben kommerziellen Navigationssystemen für Fahrzeuge oder Smartphones (visuelles oder akustisches Feedback) gibt es vermehrt Systeme, die über integrierte Sensoren taktile Signale (haptisches Feedback) empfangen können, wie zum Beispiel Armbänder. Dabei wird die grafische Benutzerschnittstelle zunehmend von Touchscreens, sogenannten Natural User Interfaces (NUI) abgelöst. Natural User Interface verlangen nicht nach Maus und Tastatur zur Steuerung, sondern ermöglichen eine natürliche (spontane) Ein- und Ausgabe über Sprache, Gesten oder Bewegung (Gesture oder Motion Tracking Interfaces). Die Eingabe beziehungsweise die Interaktion kann auch auf Augen oder gar Hirnströme ausgedehnt werden. Erste Forschungen zu erweiterten Eingabeformen wie Gesten oder Sprache wurden in den 1970er-Jahren mit dem Datenhandschuh (Sayre Glove) von der Architecture Machine Group am Massachusetts Institute of Technology (MIT) initiiert. Eines der ersten Natural User Interfaces war das iPhone von Apple. Als das Smartphone 2007 auf den Markt kam, sicherte sich Apple durch die Gestaltung einer einfach bedienbaren Benutzeroberfläche hohe Marktanteile. Die Steuerung erfolgt über einen berührungsempfindlichen Bildschirm (Touchscreen). Bisher gebräuchliche Metaphern wie Papierkorb und Schreibtisch wurden abgeschafft, die Steuerung erfolgt mittels Wischbewegung. Anfänglich setzte Apple auf Skeuomorphismus-Design, also realistische Darstellungen und die Nachahmung von bekannten Gegenständen wie ein Notizblock mit imitierter Spiralbindung. Die Steuerelemente sollten wie analoge Gegenstände und Werkzeuge aussehen, aber digital funktionieren. Als Microsoft erstmals mit Flat Design einen neuen Trend setzte (eine sehr reduzierte Form und Zeichensprache), übernahm Apple diesen Stil und setzte damit einen neuen Massstab. Flat Design setzt auf Minimalismus und Materialität; in die hierfür 
entwickelte reduzierte Formsprache wurden physikalische Gesetze wie Schatten miteinbezogen.

$\mathrm{Zu}$ den ersten Natural User Interfaces gehören auch sprachgesteuerte Systeme wie zum Beispiel Siri von Apple oder die Steuerung durch Gestenerkennung über die Berührung oder das Verfolgen von Körperbewegung. Allgemein gelten diese Interfaces als intuitiver, da die Interaktion wie mit einem realen Gegenstand erfolgt. Dadurch, dass bisherige Bezugsgrössen wie Bildschirm, Tastatur und Maus überflüssig werden, eröffnen sich neue Handlungsfelder und Gestaltungsmöglichkeiten mit interaktiven, ergonomischen und handlungsorientierten Designkonzepten. An der Universität Hannover wird an einem innovativen Navigationssystem geforscht, das über muskuläre Signale erfolgt (vgl. Pfeiffer et al. 2015). Actuated Navigation ${ }^{29}$ funktioniert über die elektronische Einwirkung und die Stimulierung des Sartoriusmuskels (Musculus sartorius, Streckmuskel des Oberschenkels), die einen Impuls zur Steuerung des Fusses einleitet (Rechtsoder Linksdrehung).(Abb. 28) Die Steuerung erfolgt über das Smartphone beziehungsweise über ein im Gerät integriertes Navigationssystem. Das $\mathrm{Na}$ vigationsgerät löst über einen elektrischen Impuls bei den Nutzern die Bewegung des Muskels aus, der dadurch automatisch den zuvor im Gerät eingetippten Weg einschlägt und das gewünschte Ziel ansteuert. Ginge es nach den Forschern, könnten Fussgänger_ innen schon bald durch elektrische

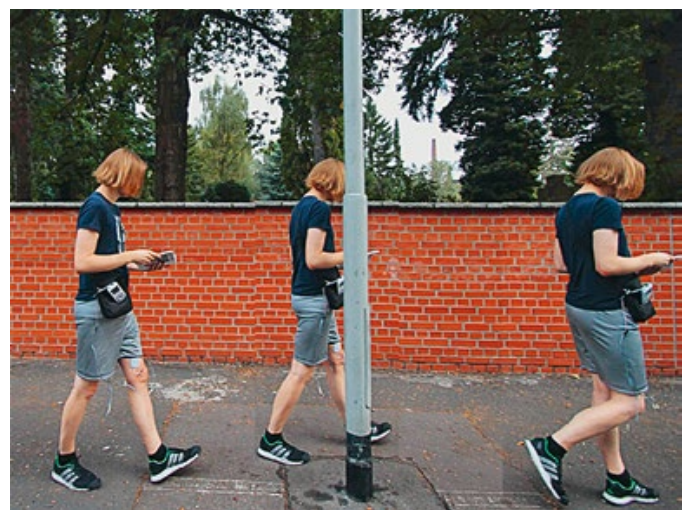

Der durch das Handy abgelenkte Fussgänger wird über muskuläre Reize automatisch um den Laternenpfosten und zum eigentlichen Ziel gelenkt. 
Muskelstimulation gesteuert werden, wodurch das Lesen einer Karte beziehungsweise der Abgleich mit der Realität überflüssig würde. Ein grosser Vorteil eines solchen Systems (Body vs. Environmental Feedback) ist, dass das Feedback über den Körper erfolgt und der Abgleich der (inneren) Karte mit der Umgebung wegfällt. Dadurch müssen keine Interpretationen vorgenommen oder Entscheidungen getroffen werden. Eine Schwäche des Systems sind sicher mögliche Zusammenstösse oder notwendige Anpassungen der Route.

\section{Interface-Gestaltung und Gestalttheorie}

Ein sicheres und Vertrauen erweckendes Interface wird schlussendlich für die Verbreitung und Akzeptanz komplexer Technologien wie automatisch gesteuerte Fussgänger oder autonomer Fahrzeuge verantwortlich sein. Die Funktionalität von Interfaces beinhaltet eine Vielzahl an Aspekten wie etwa die Wahrnehmung, die Kognition oder die Semantik. Übergeordnete Ziele sind die Funktionalität (Usability), die Interaktion (Interaction Design) sowie das Erlebnis (hierfür prägten Brenda Laurel und Nathan Shedroff den Begriff Experience Design). Die Gestalt des Interface basiert auf kognitions- und wahrnehmungspsychologischen Grundlagen der Gestaltpsychologie. Die Gestaltpsychologie wurdein den 1920er-Jahren durch Max Wertheimer (Berliner Schule) begründet. Sie geht auf den österreichischen Philosophen Christian von Ehrenfels (1859-1932) zurück, der 1890 den begriffsprägenden Aufsatz “Über Gestaltqualitäten” schrieb. Für Gestaltpsychologen basiert die Wahrnehmung auf dem Zusammenführen einzelner Teile zu einer kohärenten Figur. Der Grundsatz der Gestalttheorie besagt, dass eine ganzheitliche Gestaltung nie aus einzelnen Teilen bestehen kann, sondern immer erst im Zusammenspiel der einzelnen Bestandteile ein Ganzes ergibt. Darunter fallen Strukturen oder Ordnungsprinzipien wie beispielsweise das Wahrnehmen und Ordnen von Gestalten. Die Wahrnehmung beziehungsweise die Gestalttheorie bilden die Grundlage für die Gestaltung von Oberflächen und Interfaces.

Von der Gestalttheorie sowie aus der Gestaltwahrnehmung ( siehe auch Rudolf Arnheim Kunst und Sehen. Eine Psychologie des schöpferischen Auges, 1964) leiten sich die Gestaltgesetze ab, die bei der Gestaltung von lesbaren und harmonischen Figuren berücksichtigt werden sollten. Je nach Auffassung variiert die Anzahl der Gesetzesmässigkeiten zwischen sieben und einhundert. Das wichtigste Gesetz ist die Unterscheidung von Figur und Grund. Auf dieser 
Beziehung bauen alle weiteren Gesetzmässigkeiten auf, wie: Das Gesetz der guten (einfachen) Gestalt, das Gesetz der Nähe, das Gesetz der Gleichheit (Ähnlichkeit), das Gesetz der Geschlossenheit, das Gesetz der Erfahrung sowie das Gesetz der Konstanz. Diese Gesetze bilden - in Verbindung mit Figur (und Grund) - eine visuelle Einheit und sind ausschlaggebend für Gestaltungsfaktoren wie Geschlossenheit, räumliche Nähe, Ähnlichkeit, Kontinuität, Fortsetzung oder Symmetrie. Das Gesetz der guten Gestalt ist eines der wichtigsten Gestaltungsprinzipien im Entwurfsprozess. Dadurch werden logische Bezüge von Inhalt und Botschaft sowie die Verständlichkeit von Gliederungen und Hierarchien möglich. Oft sind es einfache Formen, die einen starken Gestalt-Eindruck erzeugen. Verantwortlich hierfür ist die Figur-Grund-Beziehung, die Geschlossenheit sowie die Gruppierung der Zeichen. Die einzelnen Objekte sollten ein Gesamtes ergeben beziehungsweise Teile davon sein und als Einheit wahrgenommen werden. Visuelle Merkmale wie Form, Grösse, optisches Gewicht, Helligkeit, Farbe, Textur, Bewegung sowie Lage der Objekte sind daher besonders bedeutsam. Sie unterstützen die Informationsverarbeitung sowie die Lenkung oder Fixation des Blicks.

\section{Interface-Kulturen und das Interaktionsparadigma}

In einer Zeit, in der Interaktion und Ubiquitous Computing paradigmatisch geworden sind, prägen Interface-Kulturen den Umgang mit neuen Technologien. Steven Johnson fasste mit Interface Culture einen Kulturbegriff, der Technologie und Kultur nicht länger trennt, sondern zusammen zu denken versucht. Seine Beziehung von "Form und Inhalt, Medium und Botschaft, Technik und Kunst " (1999: $11 \mathrm{f}$,, zu Interface-Kulturen siehe auch Sommerer/Mignonneau/King 2008) ist auch für das Design wegweisend. Es sollte vermehrt um die Konvergenz zwischen Design und Technologie in Form von Interdisziplinarität gehen (siehe hierzu auch Laurel 1990; Galloway 2012; Hookway 2014 u.a.). Seit der Einführung mobiler Endgeräte ist es sinnvoll geworden, das Interface über ein Interaktionsparadigma zu definieren (vgl. Woletz 2016) und nicht länger über ein technisches System wie in bisherigen Publikationen wie zum Beispiel in Mensch-Computer-Interface. Zur Geschichte und Zukunft der Computerbedienung (Hellige 2008). In einer Kultur, in welcher alles vernetzt ist und der Computer ubiquitär geworden ist, zeichnet sich 


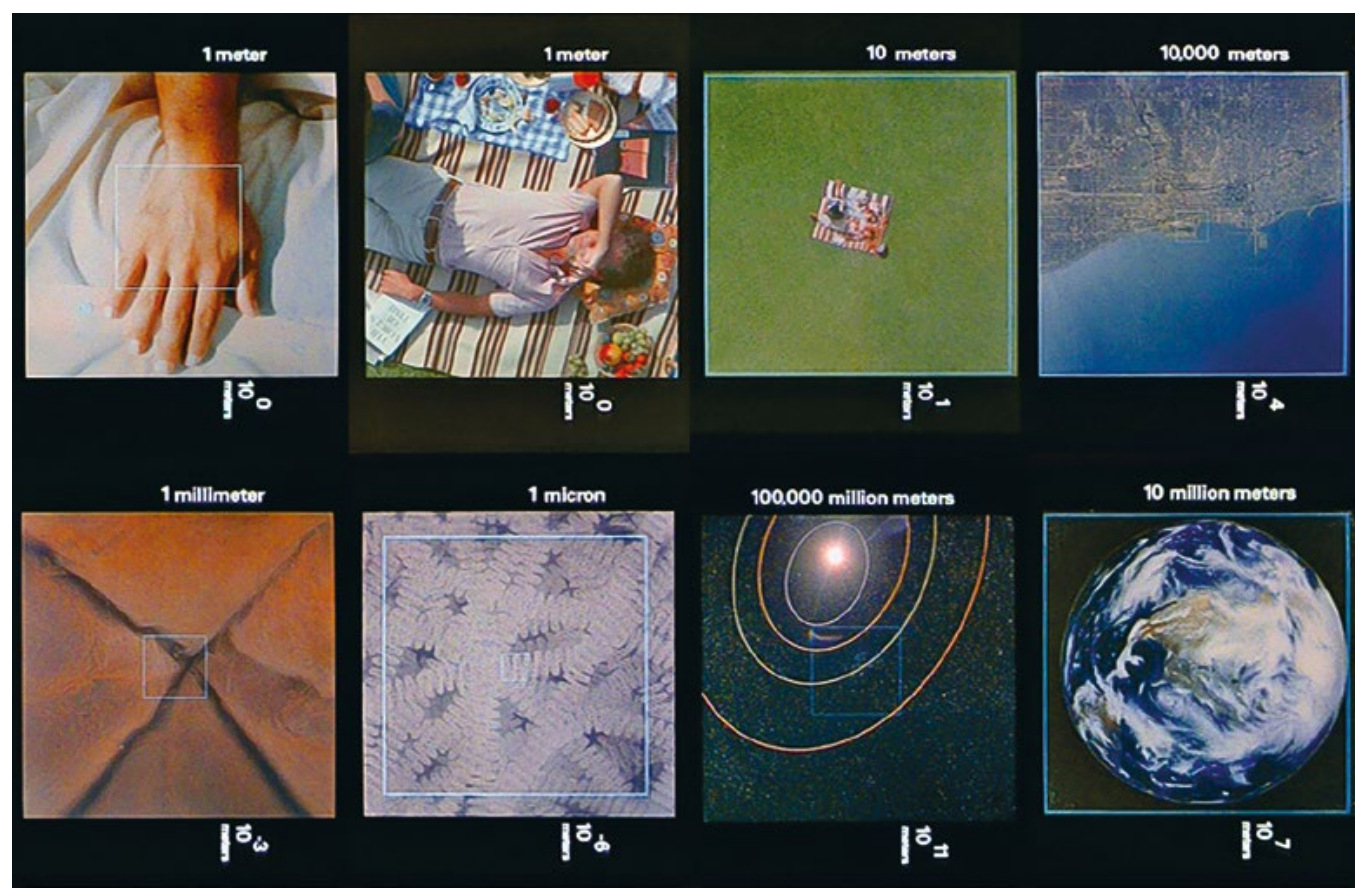

ein adäquates Interface durch eine hohe Interaktion und eine intuitive

Szenenfotos aus dem Film Zehn Hoch (1977) von Ray und Charles Eames

Benutzerführung aus. Die Verlagerung auf Interaktionsmodelle zwischen Mensch-Computer (oder System) und Raum haben Einfluss auf ein neues Designparadigma. Design kann zu einer Leitkultur werden, indem es Interaktion, Usability sowie eine Visual User Experience (Design von Informationen, Assistenz bei Aufgaben, siehe auch Garrett 2000) ermöglicht. In einem solch situierten Umfeld ist, neben der Gestaltung der Oberfläche und der Schnittstelle, das Design von Interaktion beziehungsweise ein Interaktionskonzept zentral.

Das Interaktionsparadigma beziehungsweise das Interaktionskonzept (Konzept des stufenlosen Zoomens von Google Earth) basieren auf dem Film Zehn Hoch (The Powers of Ten) des US-amerikanischen Designerehepaars Ray (1912-1988) und Charles 
(1907-1978) Eames aus dem Jahr 1977. (Abb. 29) Im Film, in dem als Ausgangsszene das Picknick eines Paars auf einer Decke in einem Park zu sehen ist, rast die Kamera ins Weltall und wieder zurück auf die Decke. Von hier zoomt die Kamera auf die Hand des Mannes und begibt sich von dort aus ins Innere des Körpers. Dabei durchläuft der Film insgesamt zehn Potenzen, sechs ins Weltall sowie in die Atmosphäre und vier ins Körperinnere. Der Film zeigt auf eine damals verblüffend technische Weise das Phänomen des stufenlosen Zoomens in einen Mikro- und Makrokosmos beziehungsweise die Reise durch Galaxien innerhalb von sieben Minuten. Die Gründer des Softwareentwicklungsunternehmens Keyhole, das später von Google aufgekauft wurde, waren begeistert, als sie den Film zum ersten Mal sahen. Um einen ähnlichen Effekt zu erzielen, kombinierte Keyhole «Satellitenaufnahmen und Computergrafik miteinander, um sehr schnell und übergangslos zwischen Erde und Weltraum hin- und herzuzoomen". (Brotton 2014: 612f.) Durch das Prinzip des stufenlosen Zoomens hebt sich die Distanz auf, und die Nutzer haben im wörtlichen Sinne das Gefühl zu fliegen. Versteht man unter Interface den medialen Zwischenraum für Interaktion und nicht die blosse Darstellung von Daten und Prozessen (vgl. Woletz 2016: 163 ff.), wird die Karte insofern zur Interface-Metapher, als sie zum Medium zwischen dem Nutzer und der Welt wird. In dieser Logik kommt es zu einer neuen Anwendung der Karte. Wichtige Parameter zur Gestaltung der Interaktion sind die Materialität sowie die Umgebung der Schnittstelle und die Distanz zu dieser. 
Macht

Die neuen

Kartograf_innen 
3

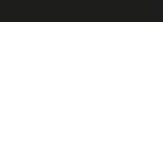




\section{Karten als Abbild verschiedener Weltentwürfe}

Die Geschichte und Semiotik der Karte, ihre Operationalisierung des Raums sowie der historische Kontext können als paradigmatisches Raummedium für den digitalen Spatial Turn herangezogen werden. Einerseits sind Karten Repräsentationsmittel, die eine möglichst objektive Wiedergabe des Raums zum Ziel haben. Andererseits ist kulturtechnisch gesehen die Medialität von Karten kein objektives Hilfsmittel, sondern Ausdruck von Macht, Eigeninteressen und politischen Ideologien. Historisch gesehen bilden im Mittelalter religiöse Zentren den Mittelpunkt mimetischer Karten. Mit der Verwissenschaftlichung der Kartografie sowie der Entdeckung und Kolonialisierung neuer Länder 
setzt sich in der Neuzeit eine allozentrische Weltsicht - Europa im Zentrum - sowie eine bis heute geläufige Nord-Süd-Ausrichtung durch. Diese jahrhundertlange Normierung wird mit Beginn der kritischen Kartografie sowie dem Postkolonialismus und den Bottom-up-Bewegungen infrage gestellt und es wird nach neuen, heterarchischen Formen gesucht.

In den 1980er-Jahren kommt es zu einem Bruch in der Kartografie. Diese, die bisher einem naturwissenschaftlichen Paradigma untersteht, öffnet sich nun. Die Kartenherstellung löst sich von der Kartografie und ermöglicht - insbesondere mit neuen Technologien und GIS-Anwendungen - eine Demokratisierung der Karte. Die Kartenherstellung verlagert sich von einer objektivierenden Tätigkeit zu einer subjektiven Aufgabe. Die soziale Dimension der Karte 
wird wichtig. Durch die Verfügbarkeit von Open-Source-Daten und Werkzeugen können die Karten nunmehr von Amateur_innen erstellt werden. Auch kollaborative Kartografien werden möglich. Gleichzeitig kommt es zu einer Machtverschiebung zugunsten von privaten Internetfirmen. Mit einem Marktanteil von rund 70 Prozent dominieren kommerzielle Kartendienste wie Google Maps und Google Earth (interaktive, webbasierte GIS-Anwendungen) den Markt. Diese monopolistische Weltdarstellung, die auch auf den gesammelten Daten des Unternehmens beruht, wird durch das Kartierungs-Commons OpenStreetMap kritisch-subversiv herausgefordert. 


\section{Die Frage nach dem Zentrum: geozentrisch oder egozentrisch}

Die Abbildung der Welt auf einer Karte ist kulturgeschichtlich aufschlussreich und gibt Hinweise auf Gebrauch und Einsatz von Karten und wie sie sich im Laufe der Geschichte verändern und wie technologische und ästhetische Innovationen ihre Gestalt beeinflussen. In jedem visuellen Weltbild findet sich eine ideelle, hierarchische Orientierung, die Ausdruck ihrer Zeit ist. Solche Weltansichten geben Auskunft über die herrschenden Macht- und Wertvorstellungen. In Europa prägten im Mittelalter die Mappa Mundi (Karten wie beispielsweise die Hereford- oder die Ebstorfer-Karte) die Darstellung des christlichen Weltbilds. Diese Karten sind schematische Abbildungen und waren weder zur Orientierung noch zur Navigation gedacht, sondern dienten der Vermittlung eines mimetischen Bilds der hierarchischen Weltordnung und des religiösen Zentrums. Solche Karten, die symbolisch-ideologische Räume abbilden, waren kreisförmig angelegt, mit Jerusalem als heilige Stadt in der Mitte, oben befand sich Asien, unten links Europa und unten rechts Afrika. Mit wenigen Ausnahmen sind diese Karten nach Osten ausgerichtet. Da sie nicht als Reisekarten bestimmt waren, war die genaue Angabe von Proportionen und Entfernungen sekundär. Es ging vielmehr darum, mit einem klar definierten christlich-religiösen Zentrum die herrschende Weltanschauung zu zeigen.

Eine genauere Darstellung der Erdoberfläche beginnt mit der Vermessung der Welt ab dem 15. Jahrhundert. Amerika wird seit der Entdeckung durch Christoph Kolumbus um 1492 auf den Karten vermerkt, es folgen die Ozeane und neue Länder und Kontinente. Aufkommende dreidimensionale Globen geben geografische Zusammenhänge und Ausdehnungen wieder und ermöglichten erste mathematische Abbildungen der Welt.

Ein eurozentristischer Blick auf die Welt beginnt in der Renaissance und mit der Vormachtstellung von Europa in der Neuzeit. Seitdem werden Karten mehrheitlich genordet. Dabei wird 1884 festgelegt, dass der Nullmeridian durch Greenwich in England geht. Bis zur Formierung einer kritischen Kartografie wird dies wenig infrage gestellt. Europa und Amerika werden als Zentrum der Welt angesehen. Bereits der griechische Mathematiker und Geograf Claudius Ptolemäus (ca. 100-160 n. Chr.) kennt eine Nord- und Südausrichtung, doch erst mit Karten, die zur Orientierung bestimmt sind, setzt sich die Nordung durch. Der eurozentristische Blick (genordete Karte mit Europa im Zentrum) ist ein antikes Erbe und baut auf der 


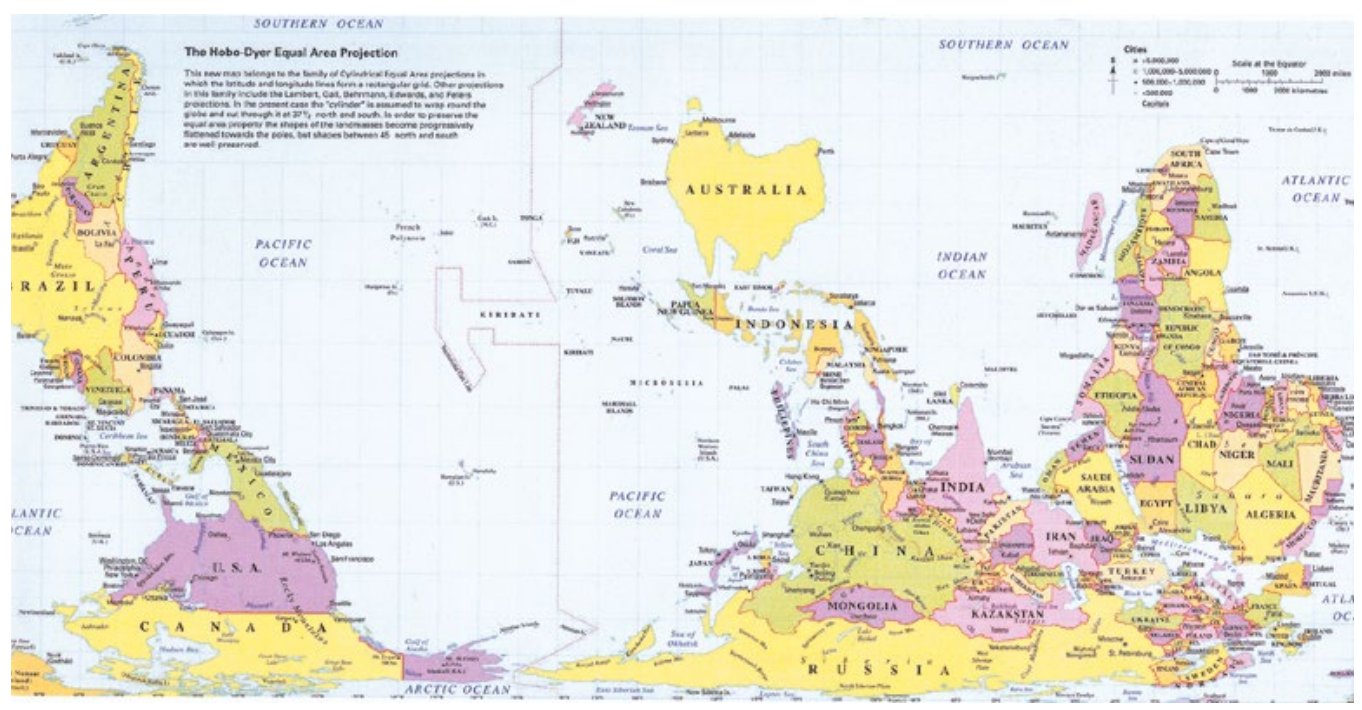

Tradition der vom Mittelmeer ausge- Die nach Süden ausgerichtete «Hobo-Dyer Equal henden Kartografie der griechisch-röArea Projection» mischen Antike auf. Mit den damals bekannten geometrischen Prinzipien der Vermessung gab es die Möglichkeit einer Nord- oder Südausrichtung. Mit der Mercator-Projektion gelingt erstmals eine annähernd exakte Berechnung (mathematische Darstellung) der Welt. Durch die Vormachtstellung der Europäer rückt automatisch Europa ins Zentrum und kann sich in der Darstellung westlicher Karten behaupten. Arabische und asiatische Kartografien stellten andere Aspekte in den Mittelpunkt: Arabische Karten zeigen oft nach Süden. Die Weltkarte "Tabula Rogeriana” von Al-Idrisi(1099-1166/80 n. Chr.) beispielsweise ist gesüdet und eine südliche Orientierung später erstellter Karten ist den arabischen Karten entlehnt. Im Zentrum der Karte steht die islamische Welt. Europa liegt hier im unteren Drittel der Karte. Auch australische Karten tauchen gesüdet auf. (Abb. 30) Bekannte Projektionen sind die flächentreue “Hobo-Dyer Equal Area Projection" aus dem Jahre 2002, 
die es sowohl in einer Nord- als auch einer Süd-Ausrichtung gibt, oder die “McArthur's Universal Corrective Map of the World" (1979) von Stuart McArthur, die Australien ins Zentrum rückt; Europa befindet sich unten rechts, Amerika unten links.

Bis zum digitalen Zeitalter basieren Karten auf einer allozentrischen Darstellung, die räumliche Informa-

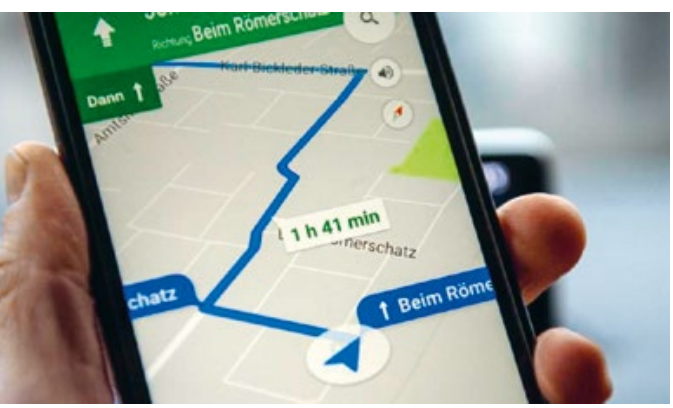
Navigation mit Google Maps auf einem Smartphone tion ist also unabhängig vom Nutzer. Dieser muss seine eigene Position zunächst auf der Karte ausfindig machen und sich im Raum orientieren (was oftmals mit einer Rotation einhergeht). Die Schwierigkeit wird deutlich, wenn man sich im Voraus mittels einer genordeten Karte über eine Stadt informiert und diese anschliessend von Norden her betritt. Dies bedingt ein gewisses Vorstellungsvermögen; muss die Karte doch gedanklich um 180 Grad gedreht werden, was sich für viele als durchaus knifflig erweisen kann. Bei einer Orientierung mittels digitaler Navigationsgeräte entfällt die Lokalisierung des eigenen Standorts. Technologische Errungenschaften führen somit auch $\mathrm{zu}$ einer Änderung von Zentrum und Peripherie. Die seit Jahrhunderten geltende geozentrische Raumordnung (Nord-Süd-Ausrichtung, Standard bei gedruckten Karten) wird im digitalen Zeitalter von einer egozentrischen Perspektive abgelöst. Auf digitalen Karten befindet sich der Nutzer im Mittelpunkt und das Ziel cläuft mit einem mit,. Im Gegensatz zu analogen Karten haben digitale Karten wie Google Maps keine Himmelsrichtungen. Zwar basieren sie auf einer NordSüd-Ausrichtung, jedoch bewegt sich die Karte mit der eigenen Fortbewe- 
gung mit. Google Maps wird horizontal am Äquator ausgerichtet, fokussiert jedoch vertikal auf das Land, in welchem die Karte abgerufen wird (vgl. Stirnemann 2018: 157). Damit setzt sich eine egozentrische Perspektive durch (auch wenn die Projektion auf einer Mercator-Karte und somit einer eurozentrischen Weltsicht entspricht). Der Erfolg von Google Earth basiert nicht zuletzt auf dieser egozentrischen Weltsicht und am - trotz des weltweiten Vernetztseins - Interesse, sich auf einer Karte selber ausfindig zu machen, wie der englische Historiker Jerry Brotton schreibt (vgl. Brotton 2014: $629 \mathrm{f}$.$) .$

\section{Google - wie ein Internetunternehmen zur Kartografie-Grossmacht wurde (Top-down)}

Mit einem digitalen Spatial Turn wird, wie bereits erläutert, der geografische Zugang zu Information beziehungsweise das Geo-Web immer wichtiger. Google hat es sich zum Ziel gesetzt, nicht nur die Erdoberfläche zu vermessen, sondern auch die Ozeane, den Mond sowie das Weltall. Das Unternehmen begründet sein Vorhaben damit, dass das bestehende Kartenmaterial nicht gut genug sei und es bisher noch kein entsprechendes Material gebe. Die Absicht dahinter und die Erstellung von gutem Kartenmaterial ist für Entwicklungs- und Schwellenländer sicher berechtigt. Kritisch zu hinterfragen ist das Monopol, das hinter dem ambitionierten Kartografie-Projekt von Google steht. Um eine gute Karte von Grund auf neu zu erstellen, braucht es Expertise, eine grosse Datenmenge sowie eine hochwertige Infrastruktur. Ein milliardenschwerer Konzern wie der GoogleMutterkonzern Alphabet verfügt über die notwendigen Ressourcen. Damit baut Google Maps nicht nur seine Vormachtstellung aus, sondern entscheidet letztlich auch über die Inhalte seiner Karten und kartografischen Online-Dienste, zu deren Erstellung das Unternehmen Satellitenbilder, Karten von Fremdanbietern, Street-View-Aufnahmen sowie die eigene Suchmaschine nutzt. Mittels Satellitentechnik ist eine genauste Vermessung der Erdoberfläche möglich geworden, aber die Frage nach der kulturellen Implikation bleibt. Die Bestrebungen, ein möglichst korrektes Abbild der Erdoberfläche zu schaffen, wird spätestens seit der kritischen Kartografie und aus subversiver Sicht angezweifelt. Die Qualität der Karte ist nicht nur abhängig von einer möglichst detailgetreuen Abbildung, sondern auch von seiner sozialen Dimension. Da Google seinen Umsatz haupt- 
sächlich mit Werbeeinnahmen erwirtschaftet, verfolgt Google Maps nicht einen rein kartografischen, sondern vielmehr vor allem einen kommerziellen Zweck. Das heisst, die Repräsentation der Wirklichkeit ist manipulativ oder mangelhaft und auf die eigene Suchanfrage optimiert. Zugespitzt bedeutet das, dass das Weltbild eine kommerziell geprägte Darstellung ist. Google Maps kann daher auch mit einem Online-Werbeverzeichnis verglichen werden, und die Karteninhalte und Wegrouten orientieren sich an der vorhergehenden Suchanfrage, den Einstellungen sowie am Standort der Nutzer. Mit der Standortangabe kann punktgenaue Werbung /Ökonomisierung von Daten) geschaltet und eine individualisierte Karte zur Verfügung gestellt werden. Dadurch sammelt Google Daten und kann über das Karten-Interface seine Suchmaschine als Schnittstelle zu seiner Datenbank positionieren. Im Jahr 2017 hat der Europäische Gerichtshof ein wegweisendes Urteil gefällt, bei dem Google zu einer Busse in Höhe von 2,42 Millionen Euro wegen Wettbewerbsverzerrung im Online-Shopping verurteilt wurde. Dem Internetunternehmen wurde vorgeworfen, seine Monopolstellung auszunutzen und nicht alle verfügbaren Resultate anzuzeigen. Google argumentiert bisher salopp gesagt so, dass man den Nutzern das anbieten wolle, was sie eben suchen würden. Konkret heisst das, dass eine selektive und personifizierte Auswahl angeboten wird, die nicht unbedingt objektiv zu sein hat. Überträgt man diesen Gedanken nun auf die Google-Kartendienste wird klar, wie verzerrt und manipulativ die Darstellungen sein müssen.

\begin{tabular}{|l|l|l|}
\hline Google-Kartendienst & Dimensionen & Anwendungsbereich \\
\hline Google Sky View & $10^{9}$ & Planetarium \\
\hline Google Earth & $10^{4}$ & $\begin{array}{l}\text { Orbitale Perspektive, } \\
\text { Satellitenblick }\end{array}$ \\
\hline Google Maps & $10^{3}$ & Karten, Flugzeug \\
\hline Street View & $10^{1}$ & Panorama, Augenhöhe \\
\hline Google Goggles & $10^{-1}$ & Armeslänge \\
\hline Iconic View & $10^{-3}$ & Submeter View, Boden \\
\hline
\end{tabular}

Das Kartografie-Imperium von Google mit den entsprechenden Dimensionen (wahrscheinlich in Metern, Ergänzung der Autorin) 
Die Karteninhalte sowie das Kartenbild waren schon immer selektiven Kriterien unterworfen und von politischen, militärischen und ökonomischen Interessen gesteuert. Bisher lag jedoch nicht die gesamte Macht bei einer privaten Internetfirma, sondern bei Institutionen wie der National Geographic Society und der United States Geological Survey oder Geographical Association. Die Ergebnisse der Suchmaschine von Google beruhen einerseits auf persönlichen Suchanfragen, andererseits auf allgemeinen Anfragen, sodass populäre Stichworte priorisiert und für die Nutzer sichtbar gemacht werden. Da ein Index fehlt, besteht die Zuordnung der Eingabe auf den Begriffen aus dem Internet. Dadurch werden Ein- und Ausschlüsse generiert, die darüber entscheiden, ob und welche Information auf welchen Karten erscheinen (beispielsweise ob ein Restaurant erfasst ist oder nicht). Graham \& Zook sprechen in diesem Zusammenhang auch von "selektiver Visibilität»: Mittels einer Studie stellten sie fest, dass eine Restaurantsuche in Tel Aviv auf Google Maps je nach Spracheinstellung (englisch, hebräisch oder arabisch) unterschiedliche Resultate liefert. (Abb.32) Die Unterscheidung betrifft nicht nur die Anzahl der Treffer, sondern auch die Auswahl und die Reihenfolge der Restaurants verändert sich signifikant (vgl. Graham \& Zook 2013: 93). Die Autoren der Studie sprechen in diesem Zusammenhang auch von einem digitalen Imperialismus: “Internet content, sorting algorithms and platforms, and common online practices all serve to reinforce the visibility of the already highly visible, and make peripheral voices more marginal. ") (Graham \& Zook 2007, zit.n. Graham \& Zook 2013: 79) Auf und mit Google Maps entsteht ein Zerrbild der Welt, das auf die anonyme Masse der Internetbenutzer zurückgeht. Anders gesagt: Wir sehen die Welt durch die Optik von Google. Zum ersten Mal in der Geschichte basiert die Abbildung der Welt auf Daten, die auf nicht frei zugängliche Algorithmen zurückgehen, da Google die Codes geheim hält (vgl. Brotton 2014: 633). Zugespitzt heisst das, dass Google letztlich mitentscheidet, wie der öffentliche Raum wahrgenommen wird. Unklar sind auch die Kriterien, auf welchen die Auswahl bei Google Maps beruht: Also ob und wie viel dafür bezahlt werden muss, um sichtbar zu werden.

Bezüglich Detailgenauigkeiten sowie Informationsfülle auf Karten von Europa oder Schwellenländern gibt es sehr grosse Unterschiede. So sind auf Google Maps bei gewissen Erdteilen ganze Regionen nur unzureichend kartiert. Aufgrund der enormen Menge an zu verarbeitenden Daten werden auch Sortierungssysteme (z. B. soziale Netzwerke) oder Software (z. B. PageRank) eingesetzt, die aufgrund der verfügbaren Informationen eine Priorisierung vornehmen und den Ort entsprechend abbilden ( siehe hierzu auch Graham 2005; 

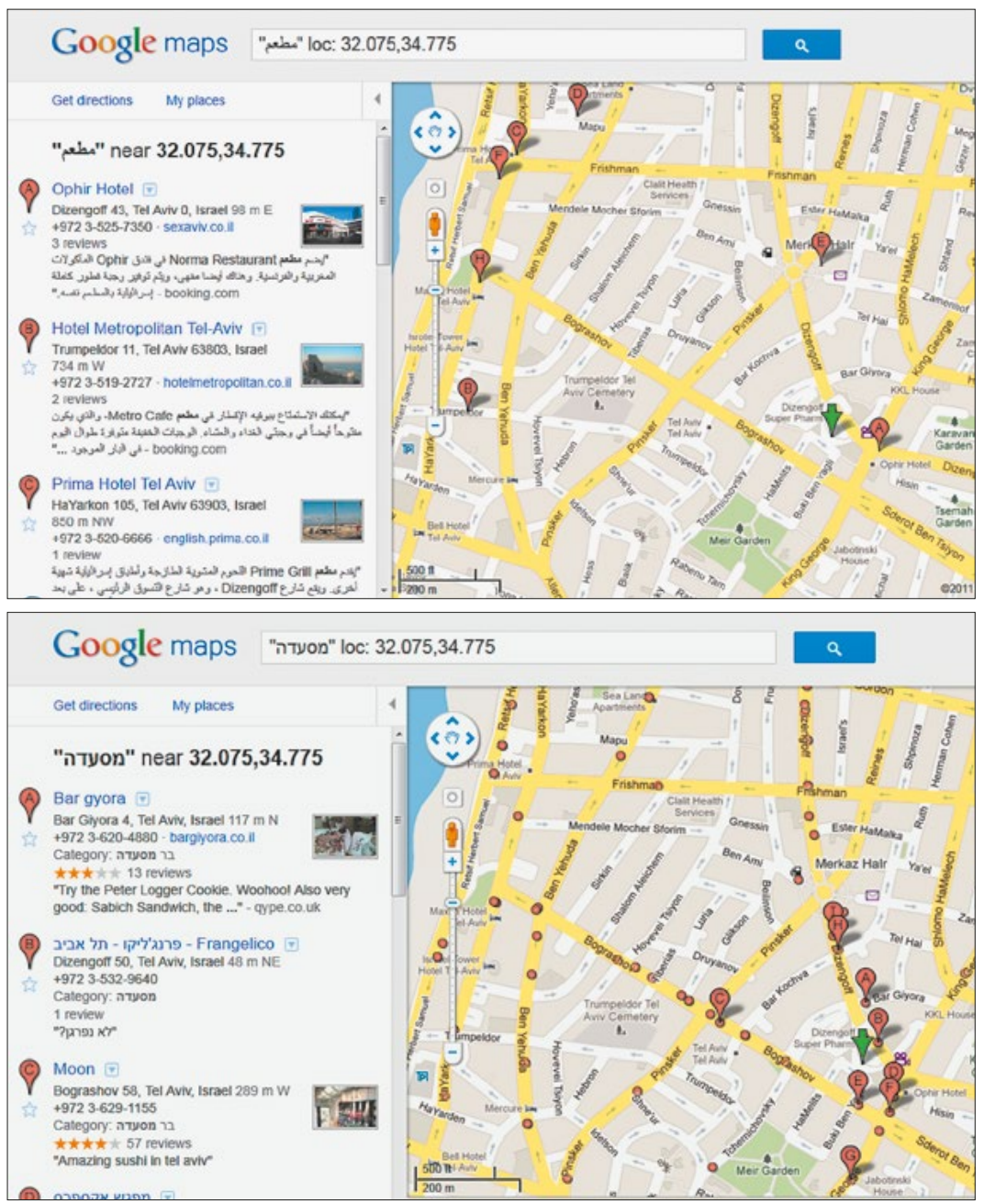

Zook \& Graham 2007b, zit.n. Graham \&Zook 2013: 77). Da Google Maps als Google Maps, Ausschnitt von Tel Aviv: Je nach Spracheinstellung verändern sich Schnittstelle zur eigenen Suchmaschine dient, bekommt das Unternehmen über die Suchanfragen Zugang zu einem riesigen Datenpool und kann so ein immenses Archiv an georeferenzierten Inhalten aufbauen. Durch das Sammeln und Speichern dieser Daten baut das Unternehmen sein Monopol laufend aus und verfügt schlussendlich über eine geografische Datenhoheit. Zugespitzt kann man es auch mit den Worten von Medienkritikern so formulieren: «We are not Google's customers: 
we are its product. We [...] are what Google sells to advertisers.” (Vaidhyanathan 2011: 3; siehe hierzu auch Lanier 2014 u.a.) In ihrer bahnbrechenden Publikation Das Zeitalter des Überwachungskapitalismus spitzt die amerikanische Ökonomin Shoshana Zuboff diese These weiter $z u$, indem sie uns als “Quellen für den alles entscheidenden Überschuss des Überwachungskapitalismus» bezeichnet, "die Objekte einer technologisch fortgeschrittenen und zunehmend unentrinnbaren Operation zur Rohstoffgewinnung”. (2018:25) Dies ist insbesondere deshalb brisant, da in Zeiten von Big Data das Sammeln und Besitzen von Daten mit einem kartografischen Machtmonopol einhergeht. Die Macht, die das Unternehmen mittlerweile hat, wird vielleicht am ehesten dadurch deutlich, dass alles, was ausserhalb der 'Google-Welt, liegt, nicht existiert. Diese Erfahrung machte auch der deutsche Künstler Aram Bartholl, der sich ein Paket schicken liess und es nicht geliefert bekam, da seine Adresse auf Google Maps nicht eingezeichnet ist und dadurch für den Auslieferer nicht auffindbar (bzw. existent) war. Dies ist umso relevanter, da Online-Karten und der geografischen Verortung von Daten eine immer grössere Bedeutung zukommen. Schätzungsweise bereits mehr als die Hälfte der Informationen auf dem Internet ist georeferenziert. Das heisst, die Inhalte sind verortet und lokalisierbar beziehungsweise mit dem physischen Raum verknüpft. Grundsätzlich hat Google grosses Interesse, alles zu georeferenzieren, um so seine Karten als Schnittstelle zu seiner Suchmaschine zu positionieren. Doch das Unternehmen baut seine marktbeherrschende Stellung nicht nur durch Geodaten laufend aus, wie bereits weiter oben beschrieben. Mit dem Aufkommen von selbstfahrenden Autos wird die Bedeutung von georeferenzierten Daten noch weiter zunehmen. “The industry has swept up cartographers, GIS specialists, roboticists, and engineers and technicians of all kinds, entangling them in what one observer calls a Billion Dollar war over maps. ") (Mattern 2017: o. S.) Karten für selbstfahrende Autos müssen über einen 3-D-Raum in Echtzeit verfügen und benötigen deshalb viel mehr, genauere und andere Daten, wie etwa die Verortung von Gegenständen mit Radar, Lidar oder Sonar und Kameras. War Google Maps bisher mehr oder weniger konkurrenzlos (es gab immer wieder kommerzielle Vorstösse wie z.B. HERE, Bing Maps oder Map.Search oder MapQuest), wird sich der Kampf um die Daten und das Kartenmonopol zukünftig noch weiter verschärfen.

Neben diesem Machtdiskurs weisen die Produkte von Google auch verschiedene Aspekte von Überwachung auf. Dadurch entwickelt sich die Geschichte der Kartografie zunehmend auch zu einer Mediengeschichte der Überwachung (vgl. Roesler-Keilholz 2013: 167; 
siehe auch Zuboff 2018 u. a.). Nachdem Google mit Google Maps und Google Earth ein Riesenerfolg gelang, floppte der neuere Dienst Street View. Das Projekt, das die Vermessung sämtlicher Strassenzüge auf einer Höhe von 2,90 Meter vorsieht, erhielt eine Flut an Datenschutzklagen von Leuten, die sich, ihre Häuserfassaden oder Autokennzeichen unfreiwillig auf den Bildern wiederfanden. Denn trotz einer Öffnung mittels Produktion und Partizipation durch die Nutzer gibt es kritische Stimme, die die Frage aufwerfen, ob "Google Earth eine Überwachung bei gleichlaufender (Entmenschlichung, des Raumes, eine Neutralisierung von Machtbestrebungen und eine Fortsetzung der Rolle von Karten als Mittel zur Durchsetzung des staatlichen und militärischen Machtstrebens » bedeuten. (Abend 2013: 196) Chad Harris und Lisa Parks gehen sogar noch einen Schritt weiter und sehen Google Earth als “eine kommerzielle, jedem zugängliche Version militärischer Überwachungssysteme und damit als weiteres technisches Mittel zur Verbreitung eines allwissenden und imperialistischen Blicks auf die Welt» (zit.n. Abend 2013: 197). Neben der Deutungshoheit ist es vor allem die Frage nach dem Interpretationsspielraum, die das Medium Karte anfällig für Manipulationen macht.

\section{OpenStreetMap - ein alternatives Kartierungs-Common (Bottom-up)}

Eine Alternative zu Googles Top-down-Formen der Kartografie ist der Common-basierte Online-Kartendienst von OpenStreetMap (OSM). Commons (dt.: Wissensallmende) sind gemeinschaftlich und gesellschaftlich getragene Projekte, die allgemein zugängliche Ressourcen nutzen und in kollaborativen Prozessen arbeiten. Sie zeichnen sich dadurch aus, dass sie auf partizipatorische Initiativen zurückgehen sowie gemeinschaftlich verwaltet und organisiert sind (vgl. Helfrich \& Bollier 2015). Der Online-Kartendienst OpenStreetMap wurde 2004 von dem britischen Informatiker Steve Coast gegründet. (Abb.33) Die Karteninhalte werden von der Crowd erstellt, zusammengetragen und zu einem Commons zusammengeführt (siehe hierzu auch Volunteered Geographic Information [VGI], z. B. Abernathy 2017). Aktuell gibt es etwa fünfeinhalb Millionen Nutzer. ${ }^{30}$ Die nutzergenerierten Inhalte werden über persönliche GPS-fähige Endgeräte wie Smartphones erhoben, anschliessend getagged und hochgeladen. Neben den über GPS-generierten Daten werden auch Satellitenbilder 


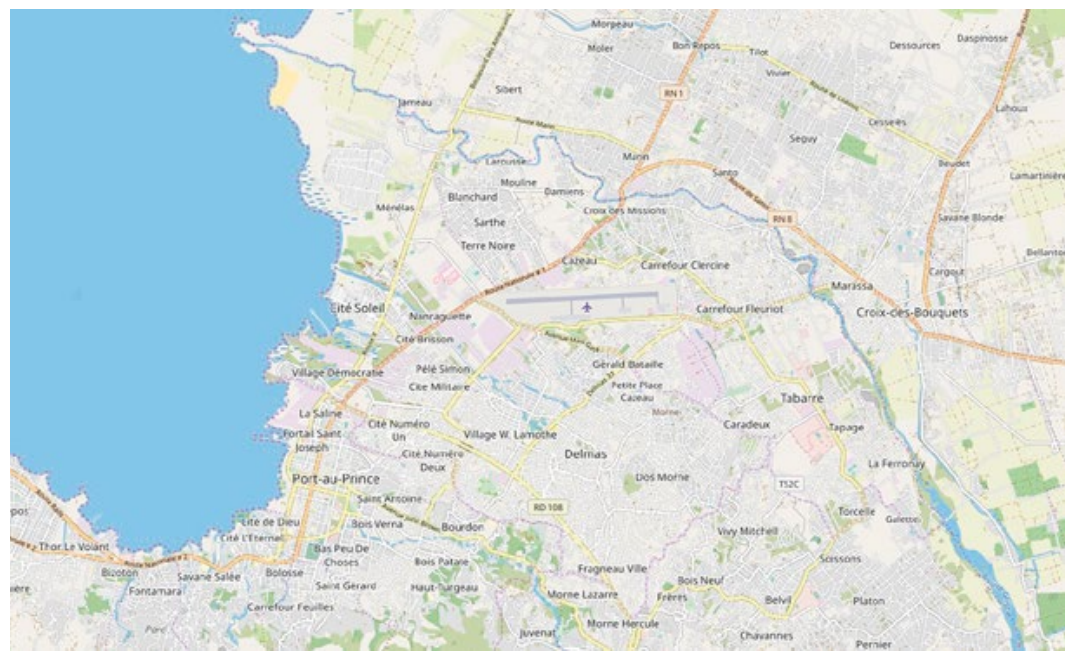

ausgewertet sowie bestehende Daten aus öffentlichen Quellen gesammelt Ein Kartenausschnitt von Port-au-Prince, Haiti, auf OpenStreetMap (2019)

(vgl. Ramthun 2012). Der kostenlose Kartendienst von OpenStreetMap basiert auf dem Wiki-Prinzip /Open Data Commons bzw. Open Database License). Das Kartenmaterial kann kostenlos und lizenzfrei weitergenutzt werden. Für die Weiternutzung werden offene Daten - Vektordaten - zur Verfügung gestellt. Denn nur offene Daten können auch problemlos überarbeitet und verändert werden. KartierungsCommons sind auch deshalb interessant, weil sie eine Alternative zu Datenhoheit, Monopol und Überwachung bedeuten. "Offene Daten sind eine wichtige Voraussetzung, die Macht der Algorithmen demokratisch einsetzen zu können.”(Stalder 2016: 270) Die Qualität der Karten ist so gut, dass sie durchaus konkurrenzfähig zu kommerziellen Kartendiensten von Google Maps sind (auch wenn Google bisher den grössten Marktanteil aufweist). Das Projekt baut auf der Mitarbeit der Nutzer auf und reguliert, überarbeitet und verbessert sich dadurch laufend selbst. Ein Vorteil von Commons-Kar- 

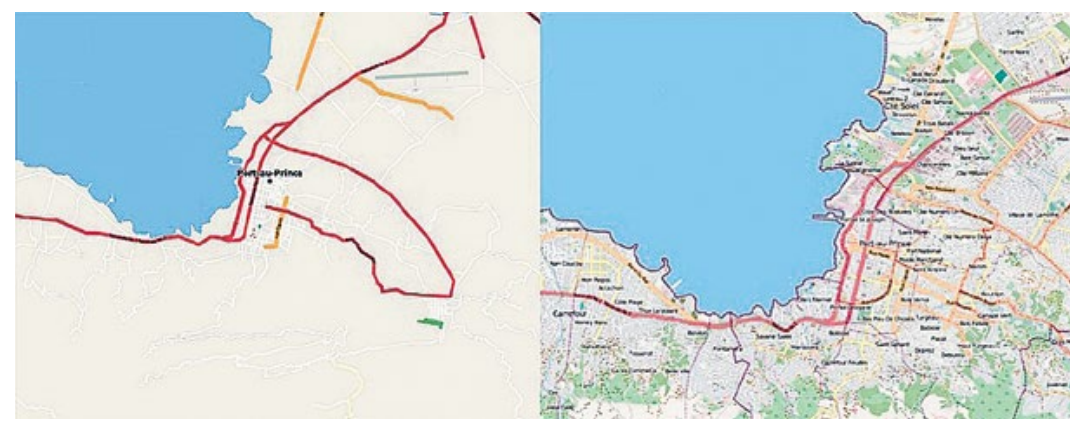

tierungen ist, dass dadurch kein Machtvakuum entsteht. Auch Google arbeitete zur Erstellung von Kartenmaterial Links: Die haitianische Hauptstadt Port-au-Prince auf OpenStreetMap am 12. Januar 2010; rechts: die Krisenkarte von Port-au-Prince 28 Tage nach dem Erdbeben kurze Zeit mit der Crowd zusammen. Das Projekt Mapmaker (2008) wurde jedoch schon bald wegen Missbrauchs eingestellt. Im Gegensatz zu Mapmaker funktioniert OpenStreetMap sehr gut, da kein Monopol und auch keine kommerzielle Absicht dahinterstehen.

Kartierungs-Commons sind vor allem in solchen Ländern erfolgreich, in welchen kein staatliches Kartenmaterial erhältlich, der Bedarf an qualitativen Karten jedoch relativ gross ist (wie in Entwicklungs- und Schwellenländern). Darüber hinaus hat sich Collaborativ Mapping insbesondere in der humanitären Hilfe (z. B. im Einsatz gegen Ebola) als wertvoll erwiesen. Collaborative Mapping wie OMS oder WikiMapia zeichnen sich einerseits dadurch aus, dass sie webbasiert sind, andererseits dass sie durch die Nutzer generiert sind. Ein bemerkenswertes Beispiel ist die Haiti-Karte aus dem Jahre 2010, die die Katastrophenhilfe von Grund auf verändert hat (vgl. Meier 2015; Schranz 2018). (Abb.34) Nach dem schweren Erdbeben in Port-au-Prince wurde mithilfe von OpenStreetMap eine webbasierte interaktive Krisenkarte erstellt. Bereits wenige Stunden nach dem Erdbeben tauchte ein hochauflösendes Sa- 
tellitenbild der Region auf, und mehrere hundert Freiwillige verwandelten dieses in eine interaktive Karte mit lebensrettenden Informationen. Dazu wurden auf der Karte sämtliche verfügbaren topografischen Informationen und georeferenzierten Daten eingetragen wie Krankenhäuser, Wasser- und Energieversorgung, beschädigte Strassen, der Zustand von Gebäuden; es wurden alle Nachrichten und Hilferufe, die in der Zentrale eintrafen und nicht georeferenziert waren, auf Satellitenbildern geortet und auf die Karte übertragen. Die Karte wurde über Social Media geteilt und mithilfe von SMS-Nachrichten und Tweets laufend aktualisiert und ergänzt. Nach der sehr wirksamen Hilfe durch das KartierungsCommons wurde ein Humanitarian-OpenStreetMap-Team (HOT) für Naturkatastrophen und Krisensituationen gegründet / siehe auch Champan 2015). Seit der Professionalisierung der humanitären Hilfe mit OSM stehen zwei Möglichkeiten für die Unterstützung zur Verfügung: einerseits das Erstellen von Karten aus Satellitenbildern durch Freiwillige, andererseits die Ausbildung und Bereitstellung von OSM-Netzwerken für gefährdete Länder. Im Weiteren setzt sich die Organisation für freie Geodaten (Open Source und Open Data Sharing) zur Rettung von Leben bei humanitären Katastrophen und zur Verbesserung von Lebensbedingungen ein und verzeichnet ein sehr schnell wachsendes Kartografie-Commons.

\section{Kritische Kartografie und Geopolitik}

Ausgehend von der kritischen Kartografie und entlang der postkolonialen Diskussionen und der damit einhergehenden Dekolonisation beziehungsweise Bottom-up-Bewegungen geriet das gängige eurozentrierte Weltbild sowie eine Nord-Süd Ausrichtung von Karten ins Wanken. Ausrichtung und Projektionsmethode von Karten, die automatisch Europa oder Amerika in das Zentrum stellen und dadurch andere Weltansichten ignorieren und nicht okzidentale Strukturen abwerten, werden infrage gestellt und kritisch hinterfragt. J. B. Harleys Aufsatz "Deconstructing the Map" von 1989 in der Zeitschrift Cartographica war bahnbrechend und wegweisend für eine neuere Kartografie. Der Artikel begründete einen neuen Trend zu einer kritischen Perspektive innerhalb der Disziplin. Wie in der Raumdebatte werden Aspekte der Subjektivität, der sozialen Dimension sowie ästhetische Phänomene wichtig und begründen eine Wende zu einer kritischen Haltung. Harley hinterfragte die bis dahin allgemeingültige Auffassung, dass Karten wissenschaftlich objektiv sind, indem er sie als 
"socially constituted image» und “inherently political» beschreibt. Bereits 1943 tauchen in diesem Zusammenhang zwei bemerkenswerte Karten auf: die "Dymaxion Map» des amerikanischen Designers und Architekten Richard Buckminster Fuller (1895-1983) sowie die "América Invertida” des uruguayisch-spanische Künstlers Joaquín Torres García (1874-1949). Beide stellen das gängige Weltbild radikal infrage, indem sie Projektion, Ausrichtung und Darstellung hinterfragen und verändern. Der als Designer und Architekt bekannt gewordene Fuller war überzeugt, dass es möglich sei, eine allgemeingültige Karte zu entwerfen, die nicht auf kulturellen und elitären Konstrukten basiert (welche in der Geschichte der Kartografie die jahrhundertlange europäische Vormachtstellung mit Europa im Zentrum wiederspiegelt). Seine "Dymaxion Map" (Dynamic Maxium Tension oder Ion) erscheint 1943 als Beilage im Life Magazin und kann herausgetrennt und zu einem Körper gefaltet werden. (Abb.35) Fullers Weltbild war nicht hierarchisch, sondern heterarchisch; es gab kein Oben, kein Unten, keinen Norden, keinen Süden. Die Karte zeigt die Welt als eine zusammenhängende Fläche, möglichst ohne Verzerrung von Grösse und Form der Landmassen und Trennung der Kontinente, umgeben von den Weltmeeren. Um eine möglichst verzerrungsfreie Projektion des Globus zu erhalten, verwendete Fuller 20 Dreiecke, die sich entlang der Kanten zu einem dreidimensionalen Vieleck (Tetraeder) zusammensetzen lassen. Dadurch lässt sich die Karte von der Fläche zum Raumkörper falten. Fuller dachte sich die Erde als einziges verbundenes Netzwerk, als ein Raum-Zeit-Kontinuum. Seine Intension war die Schaffung eines universellen Kommunikations- und Kollaborationsmediums. Der Einfluss von Fuller auf das Medium Karte sowie sein Ansatz, über die Welt in Beziehungen zwischen Kontinenten, Ländern, Meeren und Weltraum nachzudenken, ist heute aktueller denn je. Er beeinflusste damit Generationen von Designer_innen und Kartograf_innen und prägte ein neues Verständnis für die Kartografie. Zur Dekonstruktion normierter Weltbilder lohnt auch ein Blick auf die neueren Arbeiten des japanischen Architekten Hajime Narukawa oder der Grafikerin Julia Mia Stirnemann: Mit “AuthaGraph » gelang Narukawa eine fast verzerrungsfreie Projektion, für welche er den Good Design Grand Award 2016 erhalten hat. Die Karte wird seitdem in den Schulbüchern Japans verwendet. Mit einem Worldmapgenerator stellt Stirnemann ein Werkzeug zur Verfügung, das mit konventionellen Weltkarten bricht, indem Projektion und Zentrierung frei gewählt werden können (vgl. Stirnemann 2018: $175 \mathrm{ff}$. ). 


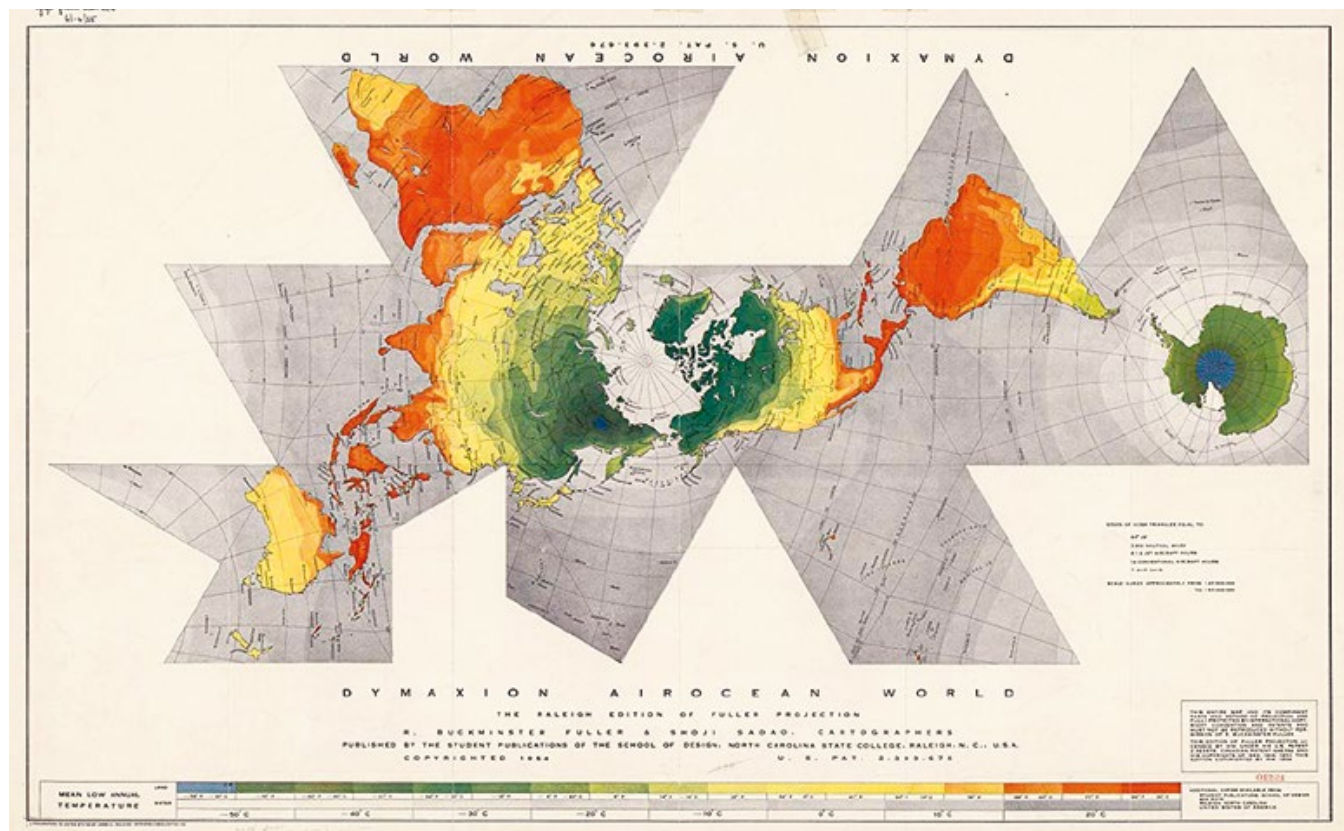

Geografische Fakten auf Karten sind aus den unterschiedlichsten Gründen Richard Buckminster Fuller: «Dymaxion Map» und Motiven schon immer unkorrekt (1943) dargestellt worden. Die Gründe sind oftmals mangel- oder fehlerhafte Angaben, Eigeninteressen, nationale Sicherheit oder politische Propaganda. Heute sind es insbesondere geopolitische Probleme, die unklare Grenzverläufe nach lokalen Präferenzen anpassen lassen. Mit der instabilen geopolitischen Lage und aktuell zahlreichen Konflikten und Interessen kommt es vermehrt zu ungenauen oder gar veralteten Karten. Laut The Guardian gibt es auf Google Maps 60 unklare Grenzverläufe weltweit. ${ }^{31}$ Obwohl Google Büros auf der ganzen Welt unterhält und seine Kartendienste in über 130 Sprachen anbietet, kommt es durch unklare Situationen immer wieder zu Konflikten. Je nach Land, in welchem federation (abgerufen am 01.10.2016). 
digitale Karten abgerufen werden, können sich Grenzen und Grenzverläufe auch in der Darstellung verändern, wie zum Beispiel bei Karten von der Krim oder von Tibet. Ende 2009 sahen Google-Maps-Nutzer in China das Gebiet Arunachal Pradesh im Himalaya als zu Tibet zugehörig markiert, Nutzer in Indien jedoch sahen es als $\mathrm{zu}$ Indien gehörend eingezeichnet (vgl. Vaidhyanathan 2011: 117). Google beteuert zwar, möglichst objektive Karten zu generieren, jedoch scheint dies ein aussichtsloses Unterfangen. Bei Unstimmigkeiten und unklaren Grenzverläufen werden auf Google Maps seit einiger Zeit die verschiedenen Grenzen mit einer gestrichelten Linie sichtbar gemacht. Falls lokale Versionen zur Verfügung stehen, werden diese als Vorlage für Namensgebung und die Darstellung von Grenzverläufen verwendet. Auch hängen die Karten jeweils davon ab, von welchem Land aus sie abgerufen und welche Spracheinstellungen verwendet werden. Beispielsweise bei der Krim: Je nachdem, ob der Ort von Russland oder der Ukraine aus gesucht wird, wird ein abweichender Grenzverlauf angezeigt. (Abb. 36) Für ukrainische Besucher von Google Maps ist die Grenze zu Russland nicht markiert, aber die innere Grenze mit dem Rest der Ukraine ist klar eingezeichnet. Anders in Russland: In unmissverständlicher Weise wird das Gebiet mit einer Grenze abgetrennt und somit als zu Russland zugehörig ausgewiesen. ${ }^{32}$ Google versucht damit, den rasanten geopolitischen Veränderungen gerecht zu wer- 
den, wohingegen bei anderen Kartenherstellern das Kartenmaterial schlichtweg veraltet ist. Beispielsweise zeigte eine Karte von Bing Maps 2014 die Krim noch zur Ukraine zugehörig.

\section{Counter, Critical, Radical und Deep Maps}

Mit der Loslösung der Karte als räumliche Repräsentation durch Eliten (wie staatliche Institutionen) sowie durch das GIS begünstigt, wurden alternative Karten und Kartierungsstrategien ${ }^{33}$ möglich. Diese Kartierungsstrategien werden unter Counter-Mapping (Nancy Peluso, 1995) oder auch Critical Mapping, Radical Mapping sowie Deep Mapping gefasst beziehungsweise Counter-Maps oder auch Critical Maps, Radical Maps ${ }^{34}$ sowie Deep Maps (Pearson \& Shanks 2001; Bodenhammer/Corrigan/Harris 2015 u.a.). Ausgehend von Denis Woods Aussage "The Cartography is Dead (Thank God!)» (2003), die er auf die traditionelle Karte bezieht, und entlang einer kritischen Kartografie hinterfragen solch alternative Kartierungsformen die traditionelle Karte. Allen Ansätzen gemeinsam ist der Versuch, tiefere Machtstrukturen offenzulegen, die soziale Dimension einer Karte $z u$ thematisieren sowie einen demokratischen Zugang zur Disziplin zu ermöglichen - im Sinne von calle können Kartografen sein. Der Begriff Counter-Mapping wurde bereits 1995 von Nancy Peluso mit dem Artikel “Whose Woods are These? Counter-Mapping Forest Territories in Kalimantan, Indonesia” (2011) geprägt. Peluso führt darin aus, dass jeder Karte eine politische Dimension zukommt und begründet dies damit, dass zum Beispiel Katasterpläne Aufschluss über Besitzverhältnisse geben (beispielsweise Waldgrenzen). Karten seien ein wichtiges Hilfsmittel gerade auch zur Durchsetzung der Rechte von sozial schwächer gestellten Personen und seien somit ein Widerstand gegen die aus Macht, Hegemonie und Top-down-Kartografie resultierenden Ansprüchen. Deshalb sei es so wichtig, dass alle in der Lage sind, Karten selbst herzustellen. In ihrer Analyse vertritt sie Kartierungspraktiken im Zusammenhang mit den Interessen und Ansprüchen indigener Völker und dem Ziel, auf annektiertes Land aufmerksam zu machen. Dabei ist ihr wichtig, dass auch Nicht-Kartograf_innen befähigt werden, Karten zu erstellen. Weiter führt Peluso die Wich-

33 Für Beispiele siehe auch www.decolonialatlas.wordpress.com (abgerufen am 28.08.2018).

34 Für eine Kritik zu dem Gebrauch der Begriffe siehe Denil 2011. 
tigkeit der Emanzipierung von GIS für die Kartenherstellung aus, welche mit einer technologischen Demokratisierung - von GIS und Satelliten-Technologien - einhergeht.

Bei Deep Mapping geht es insbesondere darum, die historische Dimension eines Orts (Sense of Place) zu entschlüsseln. Dabei interessieren die geschichtlichen Einschreibungen in einen Ort (auch Sedimente oder Schichtungen genannt), die durch das «Nebeneinander und in der Durchdringung des Historischen und des Zeitgenössischen, des Politischen und des Poetischen, des Diskursiven und des Sinnlichen " stattfinden (Pearson \& Shanks 2001, zit. n. Warf 2015: 135, Übersetzung der Autorin). Eine im Zusammenhang mit CounterMapping viel zitierte Karte ist "They would not take me there. People, Places, and Stories from Champlain's Travels in Canada" von Margaret Wickens Pearce und Michael James Hermann. (Abb. 37) Die Karte visualisiert Samuel Champlains Reise zu den Anishinabed-, Wendat-, Wabanaki- und Innu-Völkern im 17. Jahrhundert, indem auf verschiedene Medien, Orte und Zeiten (Karten, Gedanken, Tagebücher, Interviews etc.) zurückgegriffen wird. Durch die Einbettung unterschiedlicher Perspektiven /Champlains, der Inuit und der Topografie) entsteht der Versuch einer dekolonialisierenden, mehrschichtigen Karte, die nicht auf eine externe Deutungsmacht und Quelle zurückzuführen ist. Aufkommende Widersprüche, Lücken und Doppelungen sind dabei gewollt. Im Zentrum stehen der Sankt-LorenzStrom und die Gegend um die Grossen Seen (Great Lakes), um welche sich die verschiedenen Erlebnisse an unterschiedlichen Orten und zu unterschiedlichen Zeiten gruppieren.

Vorläufer subjektiv geprägter Karten sind die Mental Maps der 1960er-Jahre sowie neuere künstlerische Karten und Kartierungen, die zum Ziel haben, neue (Wissens-)Zusammenhänge darzustellen und die Karte als Machtquelle offenzulegen und zu hinterfragen. Mapping als künstlerische Forschung beziehungsweise mediales Verfahren gehört aktuell zu den wichtigsten Strategien, um komplexe gesellschaftliche Prozesse und globale Zusammenhänge aufzuzeigen, zu verhandeln und zu dokumentieren (siehe z.B. Broodthaers et al. 1997; Harmon 2004; Abrams \& Hall 2008; Harmon 2009; Obrist 2014 u.a.). Gestalterisch-künstlerische Karten und Kartierungen zur Visualisierung, Dokumentation und zum Aufzeigen kritischer, gesellschaftlicher und globaler Zusammenhänge sind brisant. Auf die beiden Arbeiten von Hans Haacke und Mark Lombardi wurde im Vorwort bereits hingewiesen. Anhand von Katasterplänen und visuellen Fragmenten untersucht Haacke die Eigentumsverhältnisse vernachlässigter Immobilien in New York. Es wird spekuliert, dass der Eigentümer - die Shapolsky et al. Manhattan Real-Estate Holdings - 


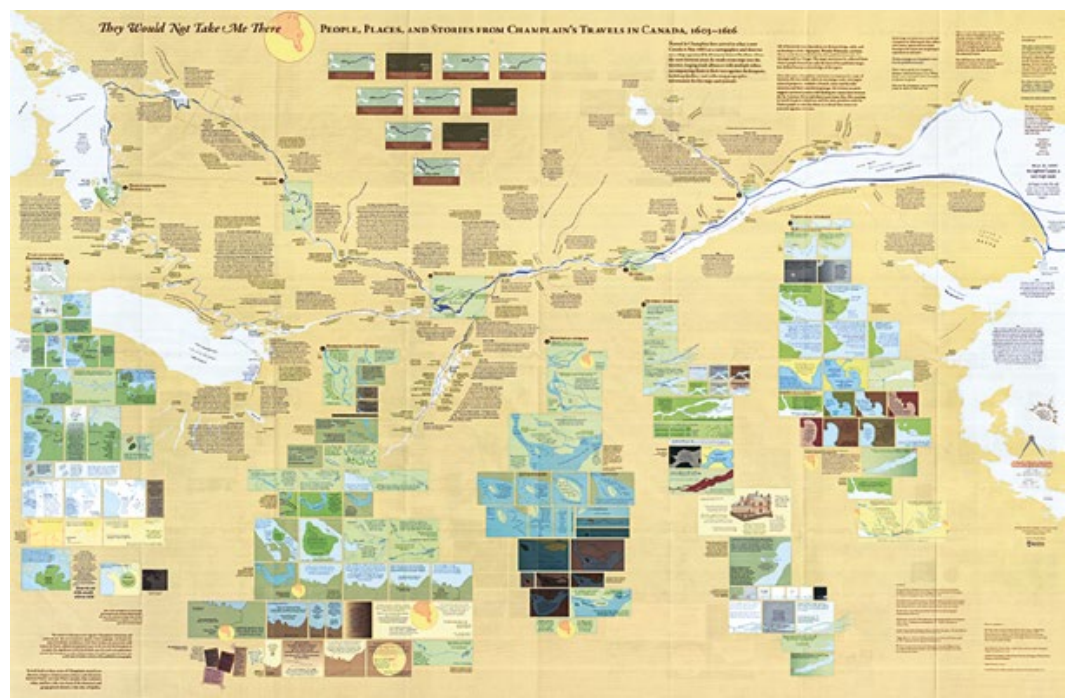

Beziehungen zum dortigen Guggenheim Museum hat, nachdem dort eine Ausstellung, in welcher das Werk von Haacke, das sich mit dem Immobilienbesitz und -spekulationen des genannten Eigentümers beschäftigt, ausgestellt werden sollte, kurzfristig abgesagt wurde. Mit “Shapolsky et al. Manhattan Real-Estate Holdings, A RealTime Social System, as of May 1 "(1971) dokumentiert Haacke die Eigentumsverhältnisse von Immobilien und damit auch die Kontrolle über grosse Gebiete der Stadt, insbesondere über Haarlem und die Lower East Side. Das

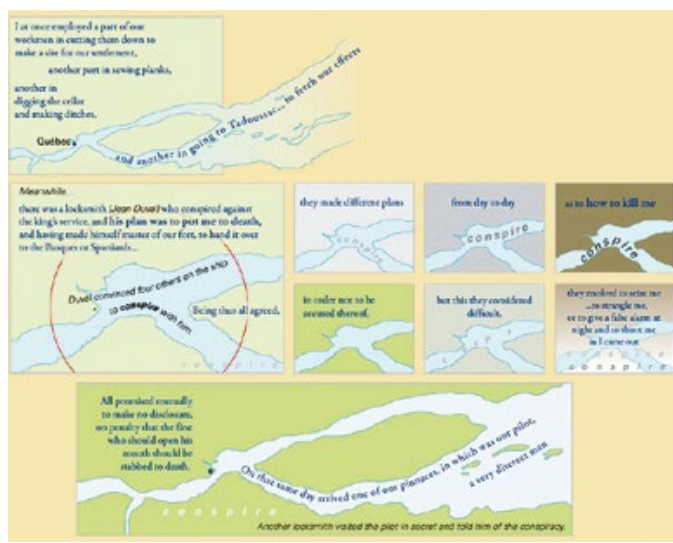

Michael James Hermann und Margaret Wickens Pearce: «They would not take me there. People, Places, and Stories from Champlain's Travels in Canada», 1603-1616 (2008) gesamte Werk besteht aus 142 Fotografien von Gebäuden, die mit unterschiedlichen Informationen versehen sind: Adresse, Art des Gebäudes, Datum des Erwerbs, Transaktionen, Eigentümer sowie geschätzter Wert. Für die 142 Gebäude sind als Eigentümer_ innen rund 70 verschiedene Unternehmen aufgeführt, wodurch die Besitzerverhältnisse verschleiert wurden, was wiederum $\mathrm{zu}$ steuerlichen Vorteilen führte. Die Arbeit thematisiert die Profitmaximierung des Immobiliensek- 
tors sowie den Gegensatz zwischen Marktanforderungen und den sozialen Bedürfnissen der Stadtbewohner_innen.

Einen ähnlichen Ansatz mit noch brisanterem Inhalt verfolgt der amerikanische Künstler Mark Lombardi (1951 - 2000) mit «BCCI, ICIC \& FAB» (1972 - 1991). (Abb. 38) Die Akronyme im Titel beziehen sich auf Banken, die zum Umfeld des internationalen Terrorismus gezählt werden, wie zum Beispiel die Bank of Credit and Commerce International. In minutiös recherchierten und mittels Linien, Kreisen und Pfeilen dargestellten Informationen zeigt er die Verstrickungen und den Filz zwischen Wirtschaft, Politik und Militär. Die narrativen Karten - oder vielleicht sollte man eher von Diagrammen sprechen verdeutlichen mittels einfachster Technik (Stifte auf Papier) die politisch und wirtschaftlich geleitete Weltordnung. Sie zeigen ein Netzwerk von Geld, Macht und Beziehungen (z. B. die der Familien Bush und Bin Laden) und sind ein Abbild einer elitären Welt. Lombardi selbst spricht von narrativen Strukturen: Informationen, welche er aus Büchern und Artikeln zusammengetragen und in eine visuelle Form gebracht habe. Die sonst isolierten Informationen würden anhand von Diagrammen und Verbindungen in einen Gesamtkontext gestellt und könnten dadurch neu gelesen werden. Einzeln würden die Informationen kaum Sinn ergeben, erst im Kontext liessen sich Bezüge herstellen. ${ }^{35}$ Auf der dOCUMENTA (13) wurden die visuellen Narrative erstmals in einem grösseren Kontext ausgestellt und für ein breiteres Publikum einsehbar. Das Werk besteht aus drei Zeichnungen und einem Archiv aus Nachrichtenmeldungen, das Skandale, Geldflüsse und Kapital in Politik und Wirtschaft beinhaltet. Die Karten thematisieren Netzwerke der Korruption und die Verbindungen von Politik mit milliardenschweren Unternehmen. Gemäss Lombardi sind alle Informationen frei zugänglich, er habe sie bloss zusammengetragen und in eine visuelle Form gebracht.

35 Siehe hierzu auch den Dokumentarfilm Mark Lombardi. Kunst und Konspiration der Regisseurin Mareike Wegener aus dem Jahre 2013. 


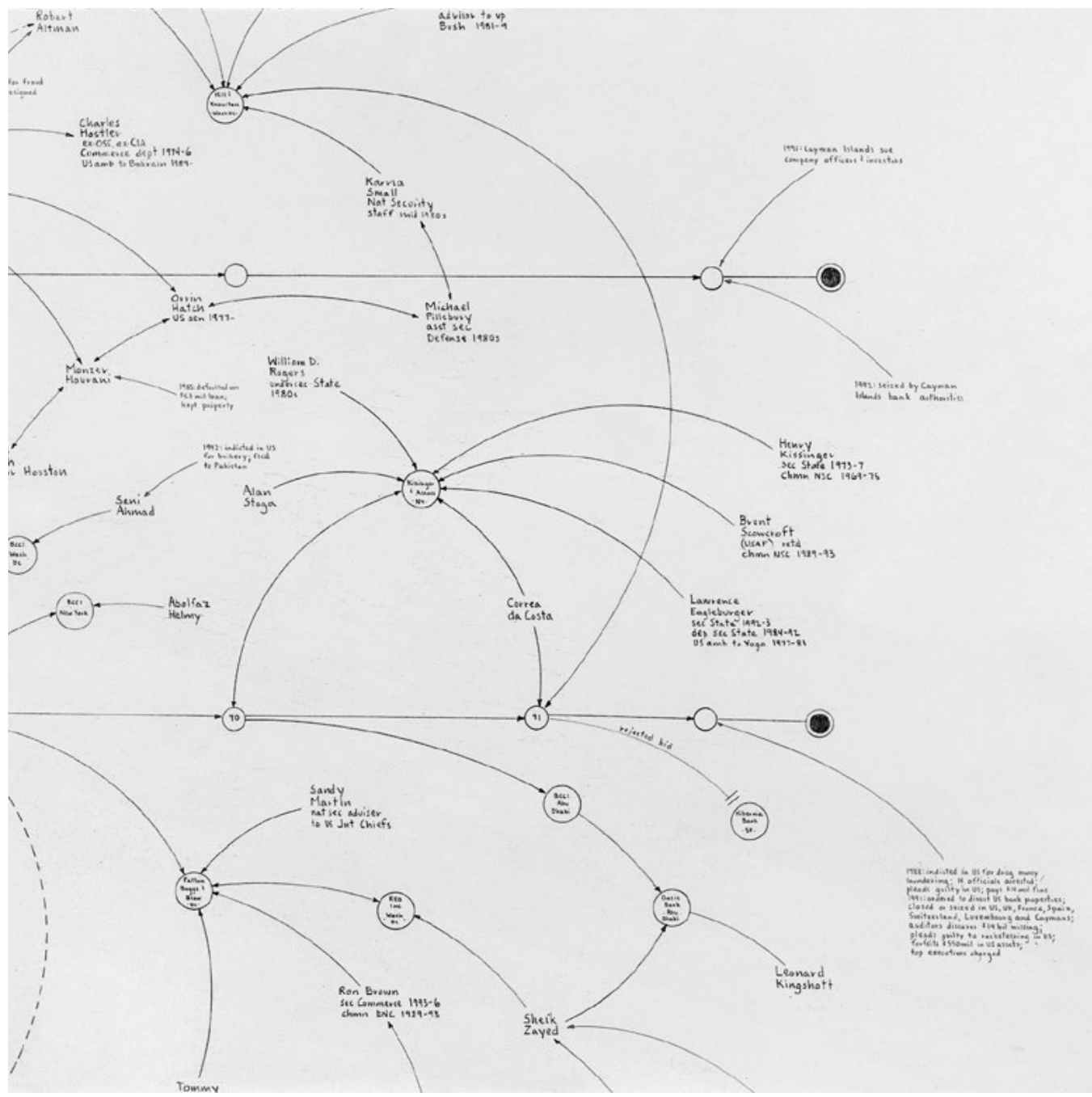

Mark Lombardi: «BCCI, ICIC \& FAB, 1972 - 91

(4th Version)» (Detail) aus der Serie BCCl, ICIC \& FAB, 1996 - 2000, Grafit und roter Buntstift auf Papier $129,9 \times 349,9 \mathrm{~cm}$ 



\section{Daten, Informationen und Wissen}

Innerhalb einer Informationsgesellschaft gewinnen Datenvisualisierungen und Datenverarbeitungen im Sinne von georäumlichen Darstellungen - insbesondere die Produktion respektive der Umgang mit der Karte - zunehmend an Bedeutung. Um georeferenzierte Daten und Informationen aufzufinden und um Zugang zu den Inhalten zu bekommen beziehungsweise um Informationen und Wissen überhaupt erst finden und/ oder verorten zu können, ist die Karte eine geeignete Ausgangslage. Die Karte als Interface möglich gemacht haben die Netzwerkgesellschaft (Castells 2001; Apprich 2015) und die immense Zunahme an georeferenzierten Inhalten. Das Smartphone hat das Verhältnis von Information zum Menschen 
verändert. Durch Technologien wie GPS, RFID und WLAN können Nutzer über ihre Mobilgeräte unmittelbar einen Bezug zur physischen Umgebung herstellen und wurden ohne ihr Zutun verort- und auffindbar. In Netzwerkgesellschaften ist zentral, wie der Zugang zur Information organisiert (strukturiert, dargestellt und gestaltet) wird. Damit einhergehend kommt es auch zu einem Wechsel von lokalem zu globalem beziehungsweise universellem Wissen.

Karten und geospatiale Anwendungen sind ein paradigmatischer Ausdruck der Informationsgesellschaft. Drei Aspekte sind für das Paradigma bezeichnend: die Anhäufung georeferenzierter Inhalte und die persönliche Verarbeitung, die Eingabe von Suchbegriffen in einem räumlichen Umfeld sowie die Erweiterung der Informationsbeschaffung in einem räumlichen 
Kontext. Durch die Informationsflut sowie mit der Komplexität und Dichte von Informationen gewinnen Informationsvermittlung und -darstellung an Relevanz. Ein schnelles und richtiges Verstehen von Daten sowie Informationen rückt in den Vordergrund. Dadurch verändert sich nicht nur die Suche, sondern es ändern sich auch die Darstellungsformen. Dieser Wechsel bringt paradigmatisch neue Suchund Sortierungsformen hervor. Die Karte wird zur Grundlage für die Informationsaufnahme, wodurch es zu einer Verlagerung der Interaktion kommt: Die Interaktion mit der Umgebung wird abgelöst durch die Interaktion mit der Karte als Umgebung. 


\section{Georeferenzierte Inhalte}

Mit den Satellitennavigationssystemen zur Ortung und Aufzeichnung der eigenen Position (Standortbestimmung) haben georeferenzierte Inhalte enorm an Bedeutung gewonnen. Dies wiederum hat Einfluss darauf, wie Nutzer mit ihrer Umgebung in Interaktion treten. Jörg Döring und Tristan Thielmann sprechen in diesem Zusammenhang auch von einer Verlagerung des WWW (wer, was, wann) zu einem WWWW (wer, was, wann, wo) (vgl.Döring \& Thielmann 2009a: 11). Verwendet werden diese Technologien stark im militärisch-ökonomischen Komplex. Es fanden sich aber auch früh künstlerische Arbeiten, wie beispielsweise jene von Laura Kurgan, die diese Technologien mit experimenteller und auch emanzipatorischer Absicht einsetzten. Eine weitere künstlerische Arbeit, die sich der technologischen Möglichkeiten der Georeferenzierung bedient, ist "Amsterdam RealTime» (2002) von Esther Polak und Jeroen Kee, die Aufzeichnungen mittels GPS als narrative Strukturen darstellen. (Abb. 39) Hierfür wurden wöchentlich zehn Proband_innen auf ihren alltäglichen Gängen durch die Stadt mit einem GPS-Empfänger ausgestattet. Die aufgezeichneten GPS-Spuren wurden in der Ausstellung Maps of Amsterdam öffentlich zugänglich gemacht und gleichen einem Logbuch der Probanden. Die Menge an georeferenzierten Inhalten führte dazu, dass der Webinhalt zunehmend räumlich organisiert und nach spezifischen geografischen Koordinaten geordnet ist. Erst dadurch konnte das Geo-Web überhaupt entstehen.

Technisch gesehen hat das Geo-Web den Umgang mit Raum und Ort revolutioniert. War früher das Browsen im Internet vor allem ein Klicken durch Listen und Hyperlinks ohne jegliche räumlichen Bezüge, werden mit neuen Technologien (wie GPS, RFID, WLAN) die gesuchten Inhalte räumlich verortet, organisiert und dargestellt. Anders gesagt wird nicht mehr nach einer Information gebrowst, sondern gezielt nach räumlichen Zusammenhängen und nach einem Standort gesucht. Suchmaschinen wie Google oder Wikipedia gewähren als Datenwächter Zugang zu den Informationen. Die Absicht der Erfinder der Suchmaschine und Firmengründer von Google, Larry Page und Sergey Brin, ist es dann auch, das gesamte menschliche Wissen zu kartieren. Gemäss Schätzungen sind mehr als 30 Prozent der Sucheingaben auf Google georeferenziert, das heisst, sie sind räumlich verortbar oder enthalten ein geografisches Element; und Google Earth soll von mehr als der Hälfte der Internetnutzer regelmässig genutzt werden (vgl.Brotton 2014: 630). Entsprechend basieren Suchergebnisse nicht mehr ausschliesslich auf alphabetischen und numerischen Kriterien, sondern auf georeferenzierten 


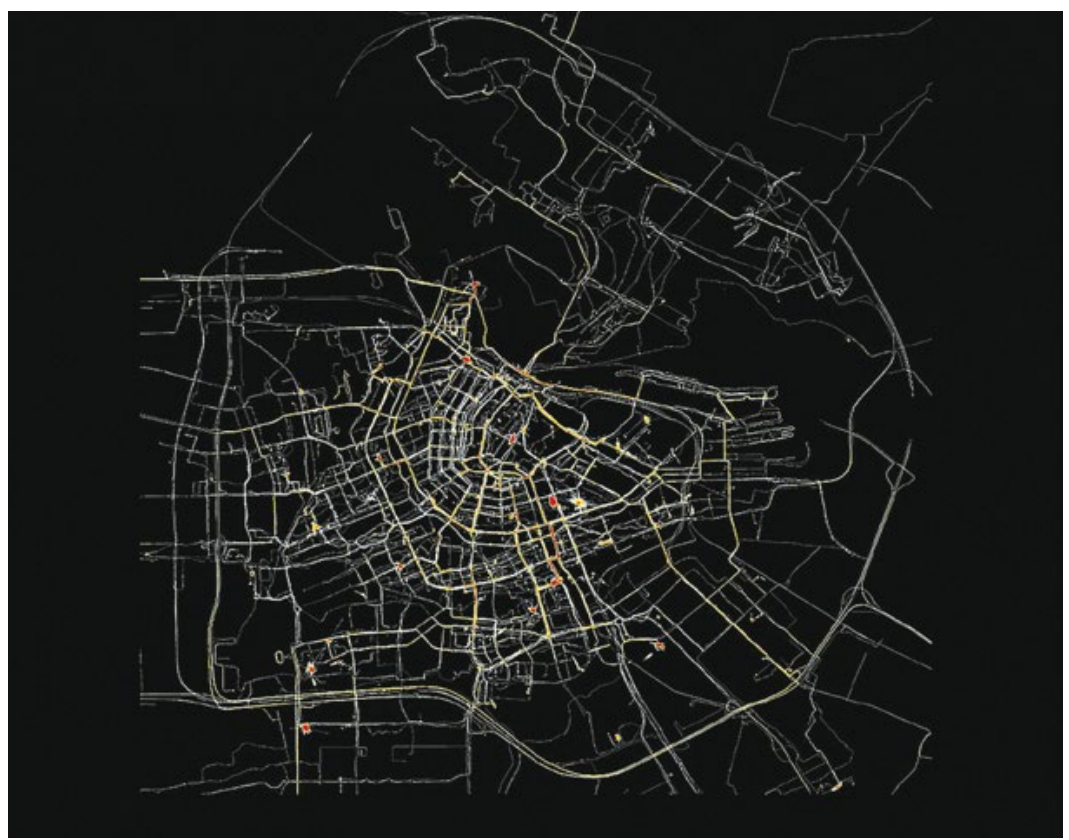

Daten und Geodaten. Ausschlaggebend für die Verbreitung georeferenzierter Esther Polak und Jeroen Kee: «Amsterdam Inhalte sind geospatiale Anwendungen RealTime» (2002) wie Sensoren, ortsbasierte Dienste oder Augmented-Reality-Applikationen. Dadurch, dass Daten verortet beziehungsweise spezifischen geografischen Koordinaten zuordbar sind, verändert sich nicht nur das Suchen, Finden und Teilen von Daten, sondern auch die Darstellungsformen verändern sich.

Viele dieser georeferenzierten Inhalte werden durch die Nutzer selbst produziert. Dies führt dazu, "dass eben nicht nur User Generated Content entsteht, sondern User Generated Spaces (UGS)", und weiter: "Raum ist nicht länger Behausung von Kontext. Umgekehrt: Vernetzte Kontexte erzeugen Räumlichkeit und widerrufen diese.” (Fassler 2009: 189) In einem solch situierten Umfeld wird die Karte zur Grundlage, um Suchanfragen zu starten und Informationen und Orte $z u$ lokalisieren. Im Unterschied zu der 
Textsuche mit einer Liste als Ergebnis ist bei der räumlichen Suche (bei gleichem Informationsgehalt) ein geografisches Bezugssystem die Grundlage. Die Inhalte werden über Standorte angezeigt (Place Markers) und sind dadurch verortbar, zugleich werden die Standorte in den Inhalt eingebunden. Durch das Anklicken von Links werden weiterführende Informationen abrufbar und räumlich angezeigt. Aber nicht nur das, auch der Blick auf den Standort ändert sich durch die (personalisierte) Raumsuche. Man sucht zum Beispiel nicht mehr nach einem bestimmten Restaurant, sondern bekommt mehrere Restaurants in unmittelbarer Nähe vorgeschlagen. Dadurch, dass die gesuchte Information von den Möglichkeiten in der Umgebung und dem Nutzerverhalten abhängig ist (Profil und vorhergehende Suchanfrage), findet automatisch eine Priorisierung der Information statt, die auf die Suchmaschinen und auf deren Algorithmen zurückzuführen ist. Auch wenn diese Algorithmen die Suche erleichtern und dadurch weniger in Erinnerung behalten werden muss, sind dies problematische Mechanismen, da Informationen selektiert werden und gefiltert daherkommen. Über die Suchanfrage gibt der Nutzer viel aus seiner Privatsphäre preis, was gezielt zur Verwendung von personalisierter Werbung verwendet werden kann. Inhalte und Produkte können nach den Vorlieben und dem Geschmack der Nutzer angezeigt werden (Product Placement).

\section{Verwandlung von Daten in Information und Information in Form}

Um Daten als Informationen zu verstehen, benötigen sie eine ،Übersetzung ins Anschauliche, Das heisst, erst durch die Formgebung werden aus Daten Informationen. Dieser Übersetzungsprozess ist Teil des Informationsdesigns, dessen Aufgabe es ist, eine Auswahl der zu vermittelnden Daten zu treffen und eine Entscheidung zur Form der Informationen zu machen. Die Visualisierung der Daten ist Teil des Gestaltungsprozesses und führt in einen Entwurf beziehungsweise in eine Überführung der Information in eine visuelle Form mit dem Ziel, unorganisierte Daten in nutzbare, verständliche Informationen zu übersetzen. Der US-amerikanische Informationswissenschaftler und Grafiker Edward Tufte ist einer der renommiertesten Designer und Wissenschaftler bezüglich Daten- und Informationsvisualisierung. Er hat insbesondere im Bereich von Diagrammen und visuellen Epistemen Pionierarbeit geleistet. Tuftes umfassendes 
Werk zur quantitativen Informationsdarstellung und seine daraus gewonnenen Erkenntnisse sind für die Informationsgestaltung wegweisend. Sein Anliegen ist es, mittels der Veranschaulichung von Daten beziehungsweise von Informationsvisualisierung Gegebenheiten korrekt wiederzugeben und (auch für Laien) verständlich zu machen. Denn nichts ist gemäss Tufte manipulierbarer als falsche oder irreführende Darstellungen von Daten. Vorsätzlich falsche Informationen oder schlecht aufbereitete Daten können zu Unverständnis und Verschleierung von Tatsachen führen. $\mathrm{Zu}$ Tuftes bekanntesten Publikationen zählen The Visual Display of Quantitative Information (2004) und Envisioning Information (2001). In diesen beiden Standardwerken weist er auf die Schönheit und Wichtigkeit von Daten hin und macht spielerisch auf die Notwendigkeit aufmerksam, grosse Mengen an Informationen verständlich darzustellen. Um Informationen in eine lesbare Form umzuwandeln, arbeitet Tufte unter anderem mit der Datendichte (Anzahl der Einträge in der Datenmatrix), der Datenkomprimierung, mit Farben sowie verschiedenen Detailstufen (von einer Übersicht bis hin zur detaillierten Struktur). Er betont auch die Wichtigkeit, die richtigen Vergleiche zu zeigen sowie valide Ziele aufzustellen. Ein Vorläufer und bemerkenswertes Beispiel der Informationsgestaltung ist die “Carte figurative des pertes successives en hommes de 1'Armée Française dans la campagne de Russie 1812 - 1813 " des französischen Ingenieurs Charles Joseph Minard (1781-1870) zum Russlandfeldzug von Napoleon um 1869. (Abb. 40) Die Karte zeigt - auf eine für damalige Zeit verblüffende visuelle Weise - verschiedene, eigentlich unsichtbare Variablen: die Grösse der Armee, ihren Standort, die Truppenbewegungen, die Anzahl der gefallenen Soldaten sowie die Temperaturen (vgl. Tufte 2004: 41). Diese Karte kann als nüchterne Infografik gelesen werden, jedoch hilft einem auf einer weiteren Ebene die Visualisierung, das Grauen, für das die Zahlen stehen, besser zu erahnen.

Mit der Ubiquität von Informations- und Kommunikationstechnologien nahmen Dichte, Komplexität und Komprimierung von Daten und Information exzeptionell zu. Deshalb wird es immer dringender, komplexe Daten auf eine angemessene und leicht verständliche Art aufzubereiten. Aber nicht nur die Darstellung von Daten und Informationen ist bedeutsam, sondern auch der Umgang mit bestehenden Daten und Informationen. Lev Manovich weist darauf hin, dass wir in einer überfrachteten Datenwelt leben und es daher nicht das Ziel sein sollte, neue Daten zu generieren, sondern bestehende Daten in einen neuen, erleuchtenden, ungewöhnlichen Zusam- 


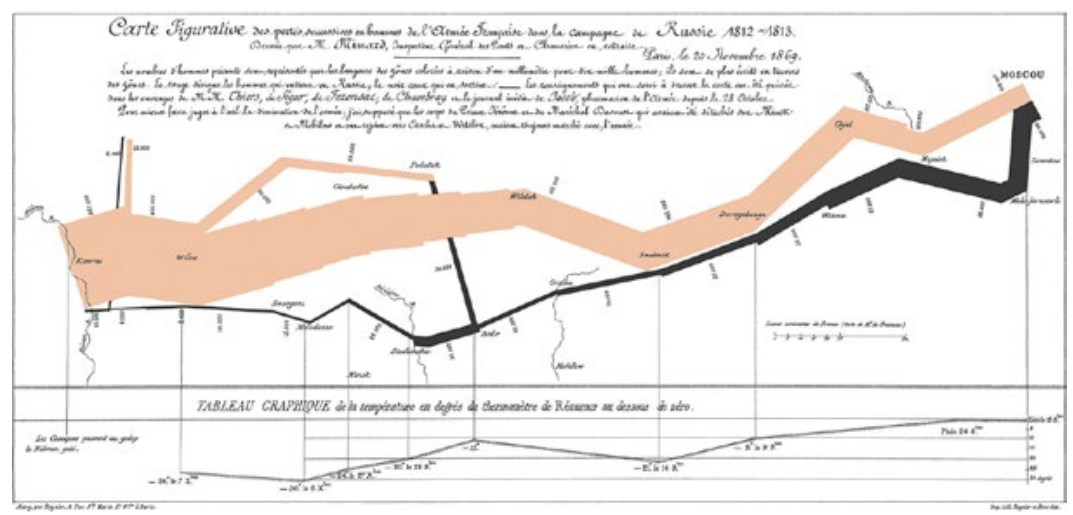

menhang zu stellen. ${ }^{36}$ Dadurch wird die Übersetzung von Daten in Information beziehungsweise Information in Form Charles Joseph Minard: «Carte figurative des pertes successives en hommes de l'Armée Française dans la campagne de Russie 1812 - 1813» (um 1869) (Text, Symbol und Grafik) noch bedeutzum Russlandfeldzug von Napoleon samer. Denn um Informationen zu verstehen, müssen diese in entsprechende Zusammenhänge gebracht und visuell verständlich aufbereitet werden. Mit der steigenden Menge an Informationen rücken daher auch vermehrt bildliche Verarbeitungsprozesse ins Zentrum. Georeferenzierte Inhalte unterscheiden sich von anderen Daten insofern, als dass sie einen räumlichen Bezug haben respektive verortet sind. Das bedeutet, dass diese Inhalte über räumliche Koordinaten verfügen. Dadurch, dass jeder Punkt - dies kann von einem Strassennamen bis hin zu einem wilden Tier fast alles sein - einem Koordinatensystem zugeordnet ist, wird der Inhalt jederzeit auffindbar. Aufgrund dieser speziellen Gegebenheit können räumliche Informationen auch bildhaft dargestellt werden. Das heisst, die Karte als Visualisierung oder zur Suchhilfe - oder eben auch der physische Raum selbst - wird zur Grundlage für

36 Eine umfassende Publikation zu diesem Thema mit dem Titel Info-Aesthetics ist im Bloomsbury Verlag seit einiger Zeit angekündigt, aber bisher noch nicht erschienen. 
die Darstellung der Inhalte im Geo-Web. Klassische Datenstrukturen, bisher in Form von Listen vorhanden, werden von kontext-bezogenen Visualisierungen abgelöst, die die Daten miteinander in Beziehung setzen und in einen räumlichen Zusammenhang bringen. Dadurch verändert sich nicht nur der dargestellte Inhalt, sondern auch die Form der Darstellung. Mit anderen Worten: Bei einer Museumssuche erscheint neben der Adresse und den Besucherinformationen zugleich ein Bild, eine Karte und ein Diagramm zu den Besuchsfrequenzen des gesuchten Standorts etc. Diese neue Darstellungskonvention Daten und Informationen in Kombination mit bildlichen Aspekten wie Diagrammen, Bildern und Karten - ermöglicht, komplexe Zusammenhänge und Prozesse sichtbar zu machen. Im Zusammenhang mit einem Netzwerk oder einer Online-GIS-Datenbank können die Daten dynamisch sein beziehungsweise jederzeit aktualisiert, personalisiert und anpasst werden. Mit einem solchen Darstellungsparadigma wird das Gestalten komplexer Daten und Informationssysteme (was Inhalt, Navigation und Interaktion betrifft) zunehmend an Bedeutung gewinnen.

\section{Die Karte als Informationsgrundlage}

Kommuniziert wurde schon immer, was sich grundlegend verändert hat, ist, wie die der Kommunikation zugrunde liegenden Informationen aufbereitet, geteilt, kommentiert und gespeichert werden. Seit Marshall McLuhan ist bekannt, dass Information erst mit der Kommunikation zum Medium wird (the Medium is the Message). Zugleich wandelt sich mit Deleuze und Guatari Information von einem linear-statischen Gebilde zu einer rhizomatisch-vernetzten Struktur. Diese medialen Entwicklungen haben sich auch im Leseverhalten niedergeschlagen. Mit der Informationsflut wird immer weniger gelesen und dafür umso gezielter nach Stichworten gesucht. Das heisst, der Selektionsprozess hat sich vervielfacht, wohingegen die Aufmerksamkeitsspanne ständig abnimmt. Der US-amerikanische Publizist Nicholas Carr, der über die Auswirkungen der digitalen Revolution schreibt, formuliert es so: Waren wir im Zeitalter von Büchern noch geübt darin, Zeile für Zeile zu lesen, werden OnlineTexte meist nur noch von oben nach unten überflogen. Das Auge orientiert sich dabei schematisch grob an der Form des Buchstabens F. Auch die Zeitdauer, die mit Lesen verbracht wird, ist kürzer geworden. Im Durchschnitt benötigen wir in etwa zehn Sekunden, um eine Website anzuschauen. Nur ausnahmsweise bleiben wir zwei Minu- 
ten oder länger auf einer Seite hängen. Dadurch wird immer weniger gelesen und der Lesevorgang wandelt sich zu einer interaktiven Tätigkeit (vgl. Carr 2010: 212 ff.). Mit diesen veränderten Gewohnheiten wurde es gleichzeitig notwendig, Information einfacher und verständlicher aufzubereiten, zu strukturieren und darzustellen. Ein wichtiges Konzept hierfür kann die Anordnung der Inhalte nach räumlichen Parametern sein, wie es beispielsweise schon Wikipedia mit Karten von OpenStreetMap ermöglicht. Georeferenzierte Inhalte können auf der Karte dargestellt, gesucht oder gefunden werden. Über eine Karte lassen sich relevante und aussagekräftige Beziehungen visuell besser begreifbar machen.

Johanna Drucker schreibt in ihrer Einleitung zu Visual Forms of Knowledge Production (2014), dass die Ubiquität von grafischen Formaten ein kritisches Verständnis für die Aufnahme und Verarbeitung von visuellen Informationen befördert habe. Dies habe zu einem neuartigen Verständnis und Gebrauch von visuellen Epistemologien und Wissensproduktionen geführt, da alte Leseformen wie Ikonografie, Layout und Diagramm/Graph nicht mehr deutlich seien (vgl.Drucker 2014: o.S.). Im Hinblick eines vermehrt räumlichen Informationszugriffs und mit einem allgemein gesteigerten Interesse an Karten / auch seit der Möglichkeit mittels Mashup Karten selbst zu produzieren), gewinnt die Karte zur Wissensproduktion sowie als epistemisches Objekt enorm an Bedeutung. Dies auch im Zusammenhang mit der Möglichkeit, Medieninhalte im Internet zu kombinieren, wie zum Beispiel die Erweiterung der Karte mit WikiEinträgen oder persönlichen Einträgen. Gerade mit geospatialen Technologien birgt das Kartieren grosses Potenzial. Mit OnlineKartendiensten kann heute jedermann Karten erstellen und über das Internet verbreiten. Dazu wird bestehendes Kartenmaterial ergänzt und nicht-kartografierte Territorien /welche in Bezug auf Sicherheit und nationale Interessen bislang weisse Flecken blieben) werden vermessen und ins Netz gestellt. Die Künstler Christoph Wachter und Mathias Jud weisen auf Karten weltweit militärische Sperrgebiete aus und versuchen, die Orte mit vorhandenen Informationen zu rekonstruieren. Dabei interessieren sie sich für "unsere eigene Imagination und die Fähigkeit unserer unabhängigen Wahrnehmung ". ${ }^{37}$ Wichtig ist hierbei, dass sie für ihre Kartierungen und Rekonstruktionen nicht als Beobachter vor Ort sind, sondern bestehendes Bildmaterial sammeln, archivieren und neu kontextualisieren. Im oben skizzierten Umfeld kann die Karte zur Ausgangslage für Interaktion, Navigation und Storytelling - kurz zur Informations- 

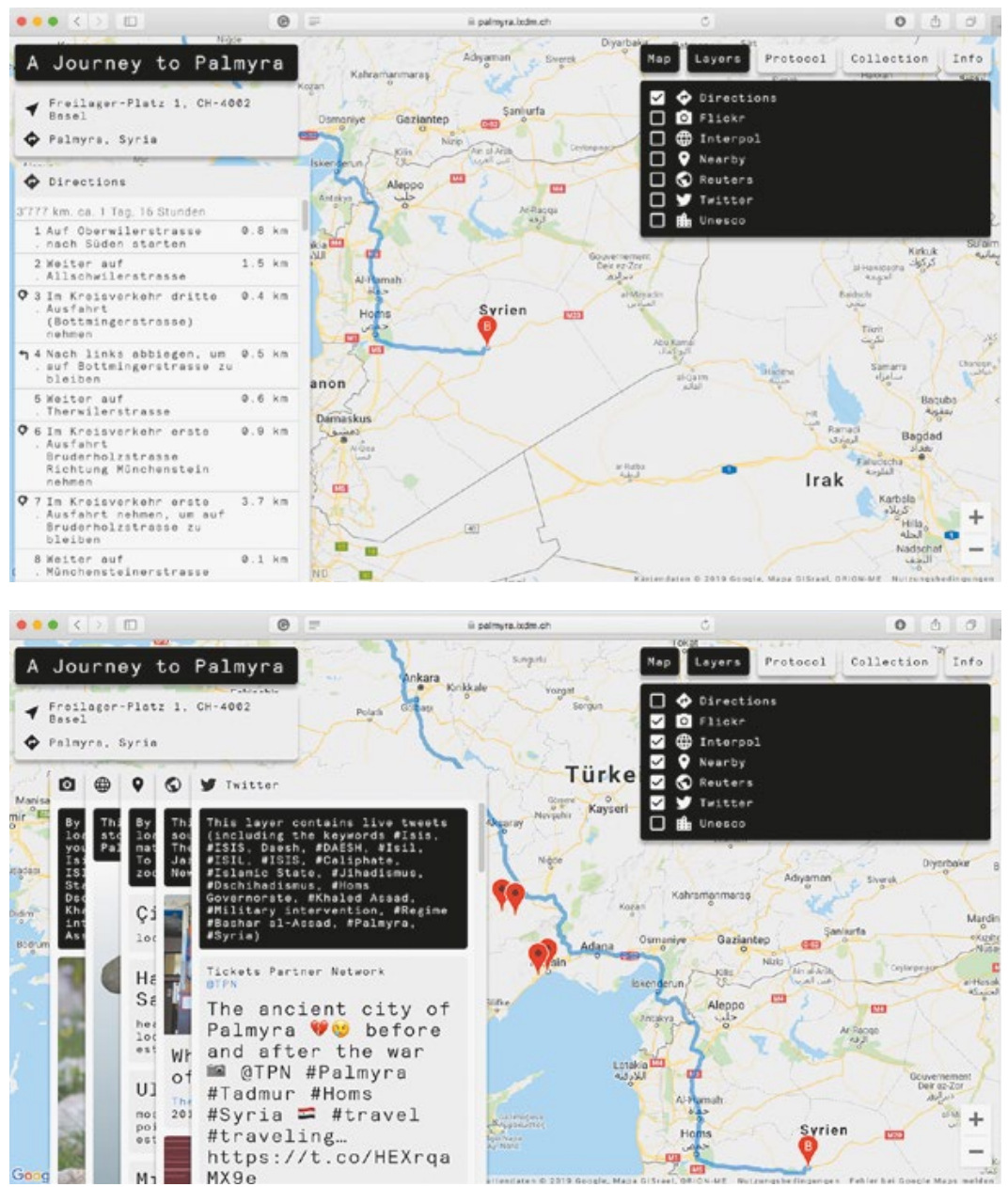

aufnahme - werden. Bei einer gezielten Christine Schranz: «A Journey to Palmyra» (2018) Informationssuche auf Grundlage einer Karte verändern sich der Lesevorgang und die Informationsaufnahme dahin, eine Karte zu verstehen und/oder deuten zu können. Dies bedeutet einerseits eine Veränderung in der Informationsaufbereitung, andererseits in der Interaktion mit der Informationsaufnahme. In " $A$ Journey to Palmyra” wird die Karte zur Ausgangslage, um eine digitale Reise zum UNESCO-Weltkulturerbe Palmyra in Syrien anzutreten. (Abb. 41) Einerseits hinterfragt die Karte unseren alltäglichen Gebrauch von Google Maps und den Anspruch auf 
Objektivität. Auf Google Map empfiehlt uns Google Directions, mit einem Auto (33 Stunden, wenig Stau) nach Syrien zu reisen. ${ }^{38}$ Google Places macht Empfehlungen zu Hotels und Restaurants in der Nähe des kulturellen Denkmals. Und dies, obwohl das durch den IS zerstörte Denkmal nicht mehr existiert und Syrien für Touristen zu diesem Zeitpunkt nicht bereisbar war. ${ }^{39}$ Andererseits wird die Karte dazu verwendet, um eine digitale Epistemologie des Orts, der Spuren auf dem Internet zum Ort, zu erstellen. Mittels verschiedener Filter können Inhalte (Flickr, Interpol, News, Twitter und UNESCO) dargestellt werden und stellen entlang der vorgeschlagenen Route räumliche Bezüge her. Die interaktive Web-Installation ist eine kritische ästhetische Praxis, die versucht, mit unseren Gewohnheiten und Ritualen zu brechen. Das Kunstprojekt umfasst zwei bekannte Bedingungen, die zwar in den Alltag eingebettet sind, aber kaum infrage gestellt werden: zum einen die mediale Berichterstattung über Krieg, zum anderen der Gebrauch von Google Maps.

\section{Wissen, Epistemologien und Information im Raum}

Mit dem Geo-Web sind grafische Schnittstellen und Informationsdarstellungen allgegenwärtig geworden. Konstitutiv hierfür sind die Netzwerkgesellschaften sowie bildschirmbasierte Medien, die cmit in die Welt rausgenommen werden, können. Auf Grundlage von Karten und kartenbasierten Interfaces können visuelle Bezüge hergestellt und der Zugang zu Daten und Informationen neu gestaltet werden. Durch mobile Geräte und mit den öffentlichen Displays entsteht ein (neuer) visueller Raum, der Einfluss hat auf das Sehen und auf die Strukturierung und Beziehung des öffentlichen Raums in Bezug auf Visualität. Beim Aufrufen oder Herunterladen von Wissen und Erfahrungen können standortbezogene Daten mit kontextbasierten Informationen kombiniert werden. Dadurch verändern sich nicht nur die Sehgewohnheiten, sondern auch die Art und Weise, wie mit Wissen und Informationen umgegangen wird. Mit anderen Worten: Standorte werden mehrschichtig, können mehrere Identitäten und Bedeutungsebenen annehmen, werden personalisiert und sind hybrid und vernetzt - im Sinne einer erweiterten Realität. Neue räumliche Visualisierungsformen entstehen, die die Verknüpfung

38 Aufgerufen am 21. März 2018.

39 Gemäss letzten Recherchen bietet der Autohersteller Clio seit März 2019 wieder Reisen nach Palmyra an. 
von Raum mit Inhalt enthalten. Das bewegte Bild prägt die Wahrnehmung; Medienfassaden und Bildschirme lösen die architektonische Struktur ab. Der deutsche Philosoph Dieter Mersch spricht gar von « multiplen Schichten oder unendlichen Palimpsesten, die nicht nur aus Häusern, Stockwerken, Straßen und Plätzen, sondern auch aus unzähligen Bildschirmen, Mobiles, Smartphones und Navis bestehen ". (Mersch 2011: 56) Dieser Raum wird in Zukunft die Computer und tragbaren Geräte überflüssig machen, an ihre Stelle treten Smart Environments. Über die Infrastruktur beziehungsweise über das Interface und die Interaktion kann eine Verbindung zum Raum und zur Technologie hergestellt werden.

Da das Wissen mit den digitalen Technologien exponentiell angestiegen ist, wird der Ein- oder Ausschluss zu Informationen immer bedeutsamer. Dieser kann im Zugang zu den Daten und der Infrastruktur liegen, wie weiter vorne beschrieben. Auf einer abstrakteren Ebene ist damit aber auch die Fähigkeit gemeint, komplexe Daten aufzunehmen und zu verarbeiten. Wie mit Tufte und Manovich herausgearbeitet worden ist, braucht es hierfür aussagekräftige Darstellungen und lesbare Vermittlungen. Das Gestalten von komplexen Informationssystemen (Inhalt, Navigation und Interaktion) wird eine zentrale Rolle im Design einnehmen. Dazu gehört auch die Entwicklung innovativer Visualisierungen. Interaktive Karten vermögen, Daten, Informationen und Wissen miteinander in Beziehung zu bringen. Darüber hinaus können künstlerische Karten das Verborgene sichtbar beziehungsweise das nicht Vorstellbare (oder auch nicht offiziell Kartografierbare) verortbar machen. In Anlehnung an Aby Warburgs bekannte Enzyklopädie und andere Formen von gesammeltem Wissen wie Archiven, Datenbanken und Bibliotheken wird auch digitales Wissen gesammelt, strukturiert und gestaltet und neue Zugänge zu Inhalt und Quellen werden erforscht. Der Künstler Trevor Paglen untersucht in seinem Langzeitprojekt, wie Wissen weltweit zugänglich wird. Im Kontext der Konflikte im Nahen Osten und Afghanistan hat er die Rolle der CIA im (Krieg gegen den Terror, in Form eines digitalen Archivs zusammengetragen. Im Zentrum stehen dabei die geheimen CIA-Überstellungsflüge von Gefangenen, die des Terrorismus verdächtigt werden. In Zusammenarbeit mit John Emerson gelang es in "Selected CIA Aircraft Routes and Rendition Flights 2001-2006” (2006) diese zensierten Flüge mit verschiedenen Quellen offenzulegen und zu kartieren. (Abb.42) Es entstand ein Kartierungsprogramm, welches die Flüge nahezu in Echtzeit sichtbar macht. Zusammen mit anderen verwandten Arbeiten wurden diese Visualisierungen unter dem Titel “Terminal Air» 2007 in einer Installation ausgestellt. 


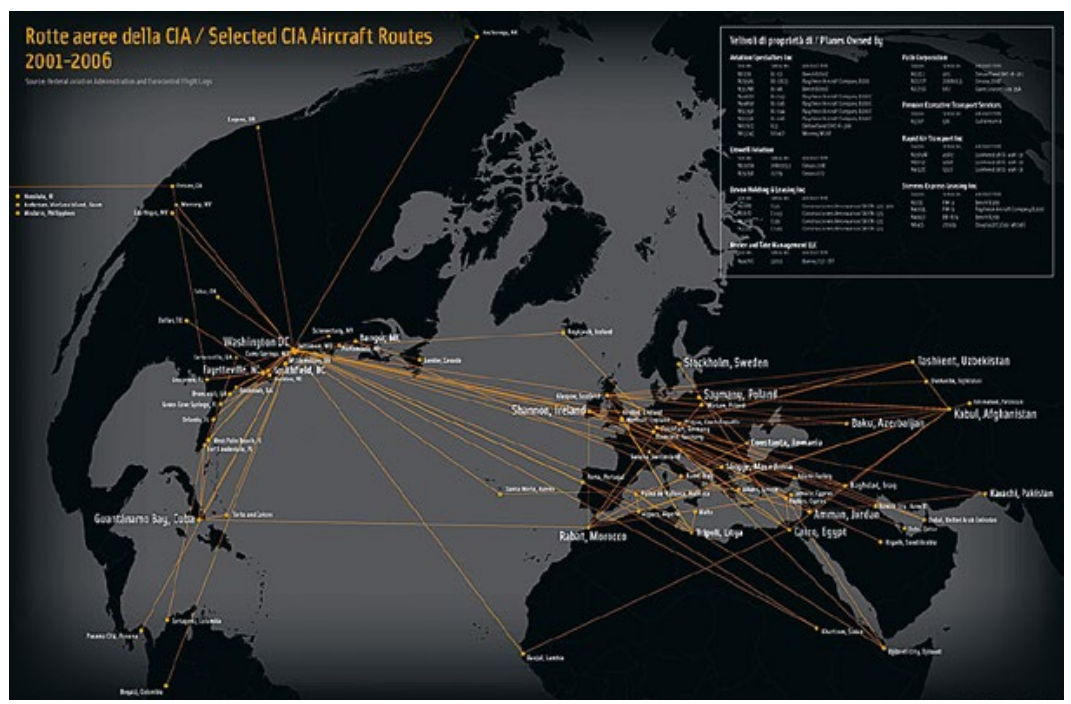

Trevor Paglen und John Emerson: «Selected CIA Aircraft Routes and Rendition Flights 2001-2006» (2006) machen mit grossem Aufwand recherchiertes Wissen um geheime CIA-Gefangenenflüge mittels Karten sichtbar. 
Navigation

Die Karte

kommt

in Bewegung 


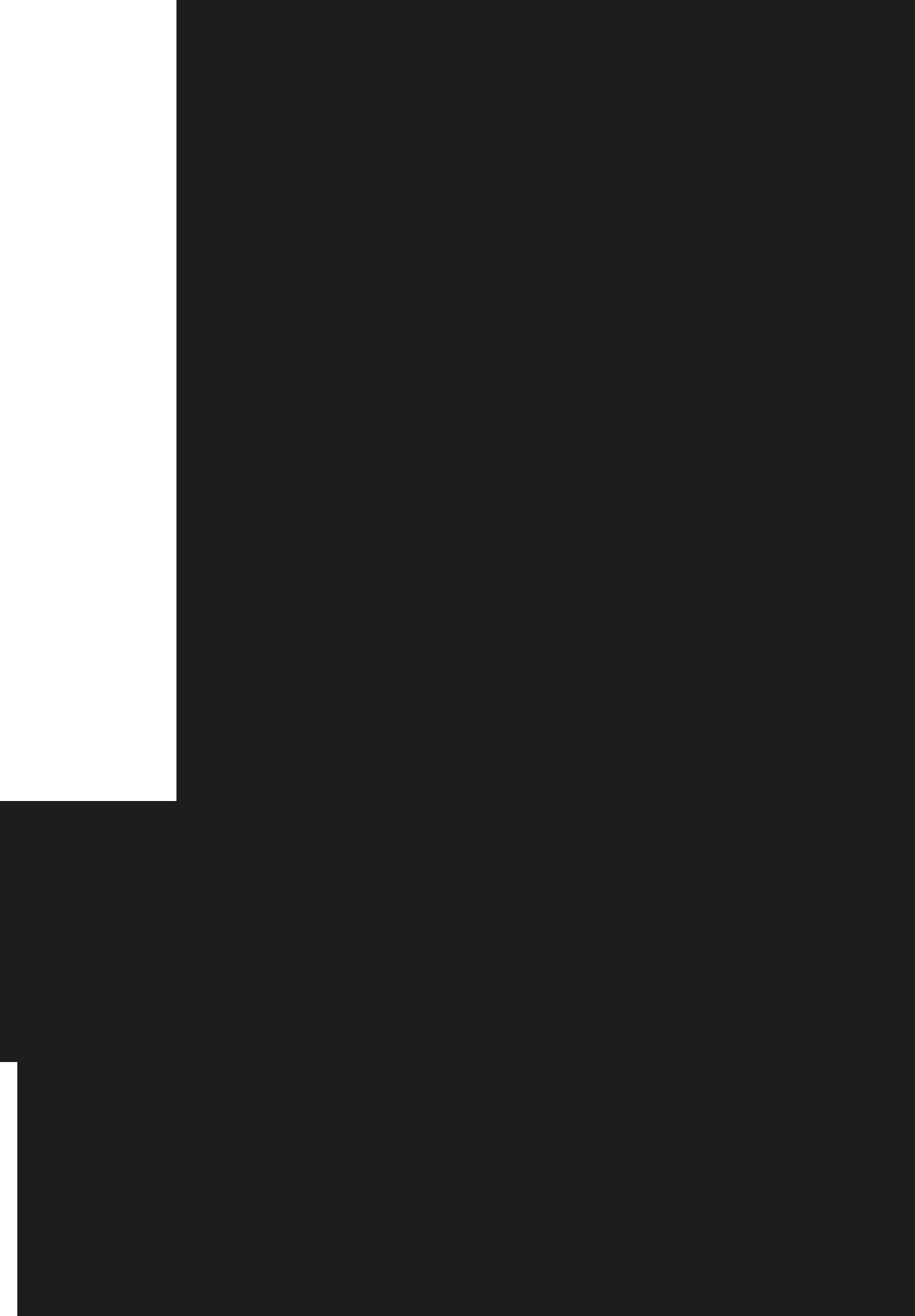




\section{Vom Kartenschauen zum Navigieren}

Wie im vorhergehenden Kapitel dargestellt, wurden die ersten Karten mimetisch gebraucht. Sie erzählten etwas über das religiöse Weltbild und die bestehenden Herrschaftsstrukturen (Deutungshoheit). Mit dem Aufkommen von Mobilität und Tourismus veränderten sich Einsatz und Gebrauch von Karten - ein navigationsorientierter Gebrauch von Karten setzte sich durch. Erste navigationsorientierte Karten waren die Seekarten (auch Portolankarten genannt), die die Handelsrouten für die Schifffahrt aufzeigten. Mit dem einsetzenden Massentourismus entstehen dann ab dem 20. Jahrhundert Reisekarten, die zur Vorbereitung und Navigation bestimmt sind. Damit rücken visuelle Systeme und kognitive Leistungen in den Vordergrund. Nicht nur die 
Darstellung des Raums verändert sich, sondern auch der Aneignungsund Lernprozess des Navigierens muss erlernt werden. Für die Orientierung sind Bezugspunkte und Systeme (Koordinaten und Karte als Hilfsmittel) bedeutsam. Darüber hinaus wird gelerntes Wissen abgerufen. Das heisst, bekannte Umgebungen, Zeichen und Symbole werden intuitiv mit der unbekannten Umgebung verglichen. Die Navigation ist eine operationale Verkettung von zielgerichteten Handlungen und Entscheidungen, welche der Orientierung und dem Sehen vorausgehen. Mit der Navigation kommt eine zweite Komponente hinzu, nämlich die der Bewegung beziehungsweise des Gehens. Michel de Certeau unterscheidet dann auch zwischen dem Sehen (Karte) und dem Gehen (Wegbeschreibung): "Sehen (das Erkennen einer 
Ordnung der Orte)", also ein Bild, oder "gehen (raumbildende Handlungen)", also Bewegung. (de Certeau 1988: 221) De Certeau weist darauf hin, dass sich Menschen bevorzugt über räumliche Bezüge orientieren. Auch gemischte Anweisungen, die ein Tun und ein Sehen beinhalten, sind oftmals einfacher nachvollziehbar und besser verständlich als reine Handlungsanweisungen. Daher werden Karten und Wegbeschreibungen oft miteinander kombiniert, sodass Mischformen entstehen. Diese Mischformen gehen über reine Handlungsanweisungen hinaus, die nur aus Richtungsvektoren bestehen, indem sie Bilder beziehungsweise Abstraktionen oder Metaphern der gebauten Welt miteinbeziehen. Das heisst, die narrative Form einer Wegbeschreibung kann durch das Bild, die Karte unterbrochen werden. 


\section{Räumliches Wissen - externe Referenzsysteme und innere Karten}

Die Orientierung ist ein kognitiver Prozess und setzt Raumwahrnehmung und räumliches Wissen voraus. Unter räumlichem Wissen werden Kenntnisse über die sich im Raum befindenden Objekte verstanden. Um Raumwissen auszubilden, ist die Fähigkeit wichtig, diese Objekte strukturieren und gliedern zu können. Das bedeutet, für das Verstehen einer Karte braucht es neben explizitem Wissen auch die Externalisierung von implizitem Wissen (Tacit Knowledge, vgl. Polanyi 1985) wie die Erfahrung oder Erinnerung. Das Gehirn besitzt die Fähigkeit, aus einzelnen Teilen (z.B. Buchstaben oder Zeichen auf einer Karte) ein Gesamtes zu erkennen, wie bereits weiter vorne erörtert wurde. Ähnlich wie der Lesevorgang, bei welchem nicht einzelne Buchstaben, sondern ganze Wörter erfasst werden, müssen auch der Sehsinn und der kognitive Verarbeitungsprozess für das Kartenlesen trainiert werden. Zusammengefasst bedeutet dies: Im Orientierungsprozess wird vorhandenes räumliches Wissen ständig erweitert und bestehendes allenfalls überprüft und korrigiert. Drei Voraussetzungen sind nötig, um eine Karte überhaupt verwenden und lesen zu können: Erstens muss der eigene Standort ausgemacht werden, zweitens muss eine Route zum Ziel ausgewählt werden, und drittens muss unterwegs die räumliche Situation mit der Karte abgeglichen werden. Für die Orientierung sind drei Konzepte bedeutsam: die sinnesgeleitete Orientierung beziehungsweise der axiale Körper (Kant 1977), externe Referenzsysteme wie Landmarken oder Himmelsrichtungen sowie eine innere mentale Repräsentation vom Raum (die auf Konzepte von Edward Tolman [1948] und Kevin Lynch zurückgeht). Für die sinnesgeleitete Orientierung sind insbesondere der Gesichts-, der Gehör- sowie der Tastsinn zentral. Beim Tierverhalten (z. B. Vögel, vgl. Hall 2008: $280 \mathrm{ff}$.) ist darüber hinaus der Erdmagnetismus wirksam, der von Menschen nicht wahrgenommen werden kann. Auf dem Erdmagnetismus baut auch der Kompass auf, der neben externen Referenzsystemen als verlässliches Messgerät dient.

Für die innere Raumrepräsentation sind kognitive Karten, sogenannte Mental Maps, zentral. Diese beinhalten das durch die Interaktion mit der Umwelt oder mit einem Hilfsmittel erworbene Wissen über die räumliche Repräsentation von Objekten. Kognitive Karten bilden sozusagen ein ,Überblickswissen, aus und ermöglichen dadurch, neben bekannten Routen auch alternative Wege zu wählen. Der Begriff geht auf den Psychologen Edward Tolman (1886-1959) 
zurück und ist in den 1960er-Jahren von Kevin Lynch massgeblich weiterentwickelt worden. Tolman konnte 1948 mit seinem bekannten Experiment und gleichnamigen Aufsatz "Cognitive Maps in Rats and Men" beweisen, dass Tiere (wie Menschen) eine mentale Karte abzuspeichern vermögen. Hierfür liess er Ratten in einem Labyrinth einen Weg zu einer Futterbox suchen. (Abb. 43) Als beim zweiten Durchlauf der Zugang zur Futterbox versperrt wurde, waren die Tiere aufgrund einer cinneren Karte, erstaunli-

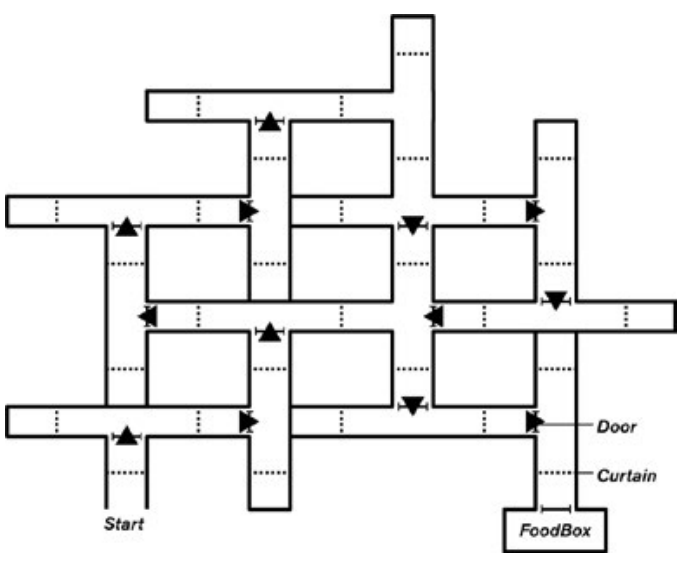
cherweise in der Lage, einen anderen Weg einzuschlagen. Für das räumliche Vorstellungsvermögen und die Orientierung ist der Hippocampus zuständig. In den 1970er-Jahren gelang es einem Team um den britisch-amerikanischen Neurowissenschaftler John O'Keefe, den Hippocampus (O'Keefe \& Nadal 1978) auszumachen. Neuere Studien zeigen, dass der Hippocampus eine Art inneres Navigationssystem im Gehirn bildet, das heisst, dass sich Karten im aGehirn ablagern, können. ZuEdward Tolman: «Cognitive Maps in Rats and Men» (1948), nachgezeichnet sammen mit dem norwegische Forschungspaar May-Britt und Edvard Moser wurde $\mathrm{O}^{\prime}$ Keefe für diese Entdeckung mit dem Nobelpreis für Physiologie oder Medizin ausgezeichnet.

Kognitive Karten können also mit Landschaften in unseren Köpfen verglichen werden und neben Bildern auch aus Erinnerungen, Gerüchen etc. bestehen. In diesem Zusammenhang soll hier an den ersten Band Du côté de chez Swann (1913, dt.Ausgabe: In Swanns Welt [2001]) von Marcel Prousts (1871-1922) Monumentalwerk À la recherche du temps perdu (dt. Ausgabe: Auf der Suche nach der verlore- 
nen Zeit [1994-2003]), erinnert werden; in diesem beschreibt Prousts in einem vielfach zitierten Abschnitt, wie der Genuss eines in Tee getauchten Madeleins bei ihm längst vergessene - und damit auch verloren geglaubte Kindheitserinnerungen wachruft. Kognitive Karten unterstützen Menschen dabei, sich in ihrer räumlichen Umwelt zurechtzufinden und Orte wiederzuerkennen, wobei sie nicht im Detail mit den realen Gegebenheiten der Umwelt übereinstimmen müssen, also keine Raumrepräsentation oder eine Karte im herkömmlichen Sinne sind. Sie gehen aus der Wahrnehmung und der Bewegung hervor und werden im Körper(-Gedächtnis) gespeichert. Unser räumliches Verhalten wird davon massgeblich beeinflusst. Da mentale Karten eine subjektive Repräsentation des Raums sind, bilden sie die Basis für Forschungen zum räumlichen Gedächtnis und der Raumwahrnehmung (bzw. zu Ordnungsstrukturen). "View of the World from 9th Avenue» (1976) des amerikanischen Illustratoren Saul Steinberg (1914-1999) gehört zu der ersten verbreiteten Darstellung einer mentalen Karte. (Abb. 44) Steinbergs Darstellung von der Stadt New York mit ihren Bewohnern, Eigenschaften und visuellen Merkmalen wurde zahlreich kopiert und auf andere Städte übertragen.

Um den inneren Bildern auf die Spur zu kommen, liess der amerikanische Architekt und Stadtplaner Kevin Lynch (1918-1984) in einer fünfjährigen Studie kognitive Karten amerikanischer Grossstädte (Los Angeles, Boston und Jersey) von Probanden_innen erstellen und befragte sie in Interviews 


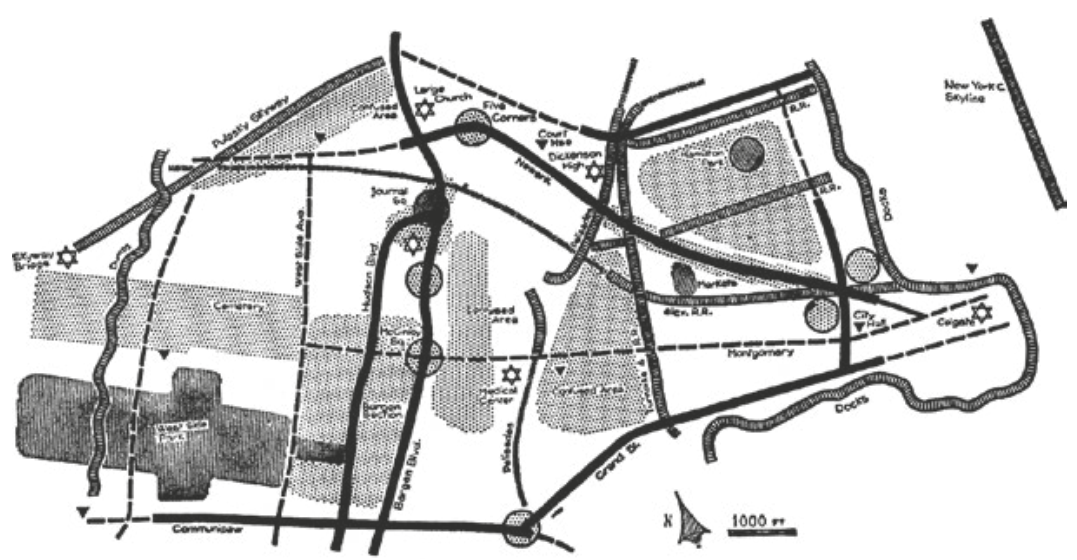

zu ihrer Raumwahrnehmung. (Abb. 45) Ziel der Untersuchung war es, eine einheitliche Kategorie zu finden, die die Kevin Lynch: «Das Bild der Stadt» (2001). Die äussere Gestalt von Jersey City entsprechend den Aufzeichnungen geschulter Beobachter räumliche Orientierung unterstützt, also der Versuch, die Raumwahrnehmung zu verallgemeinern. Dabei interessierte Lynch, wie wir uns in einer Stadt bewegen (Fortbewegung) und was wir auf dem Weg in Erinnerung behalten (Speicherung von Bildern). Aus seiner Untersuchung extrahierte Lynch verschiedene Elemente und entwickelte mit diesen eine visuelle Methode der Bildprägekraft. Diese fünf visuellen Elemente beschreibt Lynch in seinem Standardwerk Das Bild der Stadt (2001, für die engl. Ausgabe - The Image oft the City - siehe Lynch 1960; für eine Interpretation im Zusammenhang mit der Digitalisierung siehe auch Offenhuber \& Ratti 2012): Für die Wegführung kann man Linienstrukturen (Wegverläufe) folgen und/oder sich von Landmarken leiten lassen. Landmarken sind markante topografische oder räumliche Zeichen wie zum Beispiel ein Berg, eine Wegkreuzung oder ein Strassenschild, die schon von weither sichtbar sind. Lynch nennt solche natürlichen oder künstlichen Landmarken Merk- oder Wahrzeichen. Es sind 
optische Bezugspunkte, die auffällig und darüber hinaus meist gerichtet sind. Das bedeutet, dass sie oftmals nur von einer Seite wahrgenommen werden und nicht unbedingt auch von einer anderen Seite. Merk- oder Wahrzeichen werden von Wegen erschlossen. Die Wegverbindungen sind nicht zwingend direkt, sondern können aus einem indirekten Wegenetz bestehen, das durch eine Stadt oder Landschaft verläuft. Auf diesem Wegenetz kann man sich vorwärts bewegen und die räumliche Beziehung zwischen einzelnen Zeichen wird verständlich beziehungsweise sichtbar. Ein solches Wegenetz besteht beispielsweise aus einer komplexen Strassen-, Eisenbahnoder Wasserstruktur. Verbindungen oder Unterbrechungen zwischen diesen Wegen werden Knoten genannt. Knoten sind für die Wegestruktur wesentlich. Bevor eine zielführende Navigation stattfinden kann, muss die Struktur eines Orts verstanden werden. Viele Städte haben bestimmte, Bereiche genannte Abschnitte, die explizit oder implizit vom Rest der Stadt getrennt sind. Bereiche weisen oftmals eine eigene Identität auf und lassen sich optisch leicht voneinander unterscheiden. Das Wegenetz durchquert die Bereiche und verbindet diese miteinander. Unterbrechungen oder Knotenpunkte im Wegverlauf finden sich typischerweise an Brennpunkten. Brennpunkte, wie zum Beispiel eine Kreuzung oder ein Treffpunkt, sind wichtige Orientierungspunkte in einer Stadt. Die Bereiche einer Stadt beziehungsweise die Stadt selbst wird schliesslich durch Ränder begrenzt. Diese Grenzlinien verhindern das cAusfransen, und markieren die verschiedenen Bereiche. Typische Grenzlinien sind beispielsweise Flüsse, Seen oder Gebirge. Bis heute werden auf Grundlage dieser Elemente komplexe Leitsysteme für Städte und Regionen entwickelt.

Wie erwähnt, sind die Orientierung und Navigation kognitive Prozesse und eine konzeptuelle Leistung. Zur räumlichen Orientierung bedarf es neben der Auswahl einer Route, inklusive Start und Ziel, der Interaktion mit Hilfsmitteln. Anders gesagt setzt Orientierung ein dynamisches Verständnis für die Umwelt voraus. Der Orientierungssinn muss wie die Raumwahrnehmung trainiert und erlernt werden. Mit dem Orientierungssinn ist das räumliche Wissen gemeint, wie einzelne Gegenstände zueinander liegen. Zur Ausbildung des Orientierungssinnes ist die eigene Fortbewegung in Räumen zentral. So beeinflusst unter anderem, ob sich, wie etwa in den USA, Menschen vor allem mit dem Auto fortbewegen oder sich Räume und Umgebung physisch zu Fuss aneignen. Forscher_innen gehen auch davon aus, dass man erlerntes Wissen wieder verlernen kann, wenn es nicht genutzt wird. Es gibt also keinen angeborenen Orientierungssinn, wie oft behauptet und besonders indigenen Völkern zugeschrieben wird. Das Lern- und Orientierungsverhalten ist indi- 


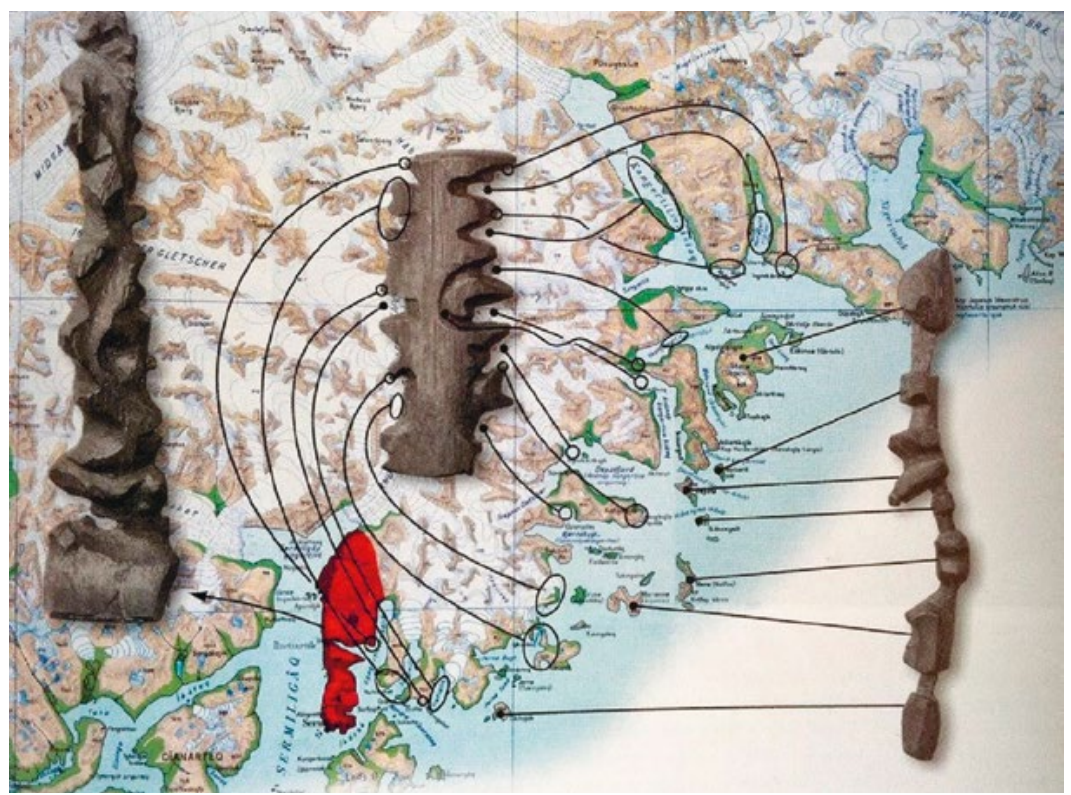

viduell geprägt und von kulturellen Einflüssen abhängig. Indigene Völker, Eine aus Treibholz geschnitzte Karte der Küstenlinie Grönlands, die von den Inuit zur Navigation die nicht über moderne Messgeräte verwendet wird oder Navigationsmittel verfügen, prägen eigene Orientierungsstrategien, die sie von Generation zu Generation weitergeben. Ein Beispiel hierfür sind die sogenannten Songlines der australischen Aborigines, die durch den britischen Weltreisenden Charles Bruce Chatwin (1940-1989), der diese in seinem gleichnamigen Roman (1987; siehe auch Chatwin 1990) beschrieben hat, bekannt wurden. Mit diesen Songlines beziehungsweise oralen Karten haben australische Aborigines eine Methode entwickelt, sich im Buschland mithilfe eines Netzwerks aus Linien und Pfaden zurechtzufinden. Ihren Weg finden sie, indem sie ein Lied singen, das sie geografisch mit der Landschaft vernetzen. Durch die Zuordnung von einzelnen Geschichten finden sie sich im Buschland zurecht: “[E]ach image, dance, and song is related to a specific place (a sacred site), a story line (myth), 
and a geographical trail that connects sites and story elements. Hyperlinks appear as users navigate, and they suggest ways to criss-cross story lines and layers of meaning in appropriate ways." /O'Rourke 2013: 119) Eine ganz andere Strategie verfolgen die Inuit in Grönland. Sie besitzen in Treibholz geschnitzte Karten, die sie zur Navigation in den Küstengewässern verwenden. (Abb. 46) Die dreidimensionalen Holzkarten zeigen neben dem Verlauf der Küstenlinie auch Inseln und Festland. Die Kartenobjekte sind haptisch und damit auch im Dunkeln lesbar.

\section{Analoge und digitale Leitsysteme}

Analoge und digitale Leitsysteme spielen im täglichen Leben eine zentrale Rolle. Ein erfolgreiches Navigationssystem unterstützt nicht nur bei der Orientierung, sondern trägt auch zur Entscheidungsfindung vor und während des Navigationsprozesses bei. Spätestens seit 1996 bei einem Brand auf dem Düsseldorfer Flughafen 17 Personen starben, dürfte bekannt sein, wie wichtig Leitsysteme sind. Aufgrund mangelhafter Signalisation konnten einige Personen im dichten Rauch die Notausgänge nicht finden und erstickten (vgl. Eibl 2002: o. S.). Für die Verständlichkeit wichtig sind dabei grafische Elemente wie Schrift, Zeichen, Farbe und Formen. Leitsysteme sollten mindestens auf dem kleinsten gemeinsamen Nenner wie Richtungspfeilen und lesbarer Typografie basieren, darüber hinaus aber für bestimmte Zielgruppen oder nach kulturellen Kontexten entwickelt werden. Die von Lynch herausgearbeiteten Kategorien - Weg, Grenzlinien, Bereiche, Brennpunkte sowie Merk- oder Wahrzeichen-haben bis heute Gültigkeit. Diese fünf Elemente unterstützen den Navigationsprozess und ermöglichen in fremden Umgebungen eine klare Hierarchisierung, speziell in Städten. Insbesondere Strassen verleihen einer Kartenansicht Struktur. Daher ist dieser Kategorie beim Entwerfen eines Leitsystems besondere Aufmerksamkeit zu schenken. Doch Lynch wollte die Städte nicht nur verständlicher, sondern auch schöner machen. Dabei bezieht er sich unter anderem auf die Gestalttheorie des ungarischen Designers György Kepes (1906-2001) (vgl.Lynch 1996). Kepes, der am New Bauhaus wirkte und ab 1967 den Bereich Visuelle Kommunikation (Center for Advanced Visual Studies) am Massachusetts Institute of Technology aufbaute, prägte massgeblich das Verständnis von Visueller Kommunikation. Kepes zufolge unterliegt Visuelle Kommunikation einem universellen Verständnis. In seinem 1944 erschienenen wegweisenden Standardwerk 


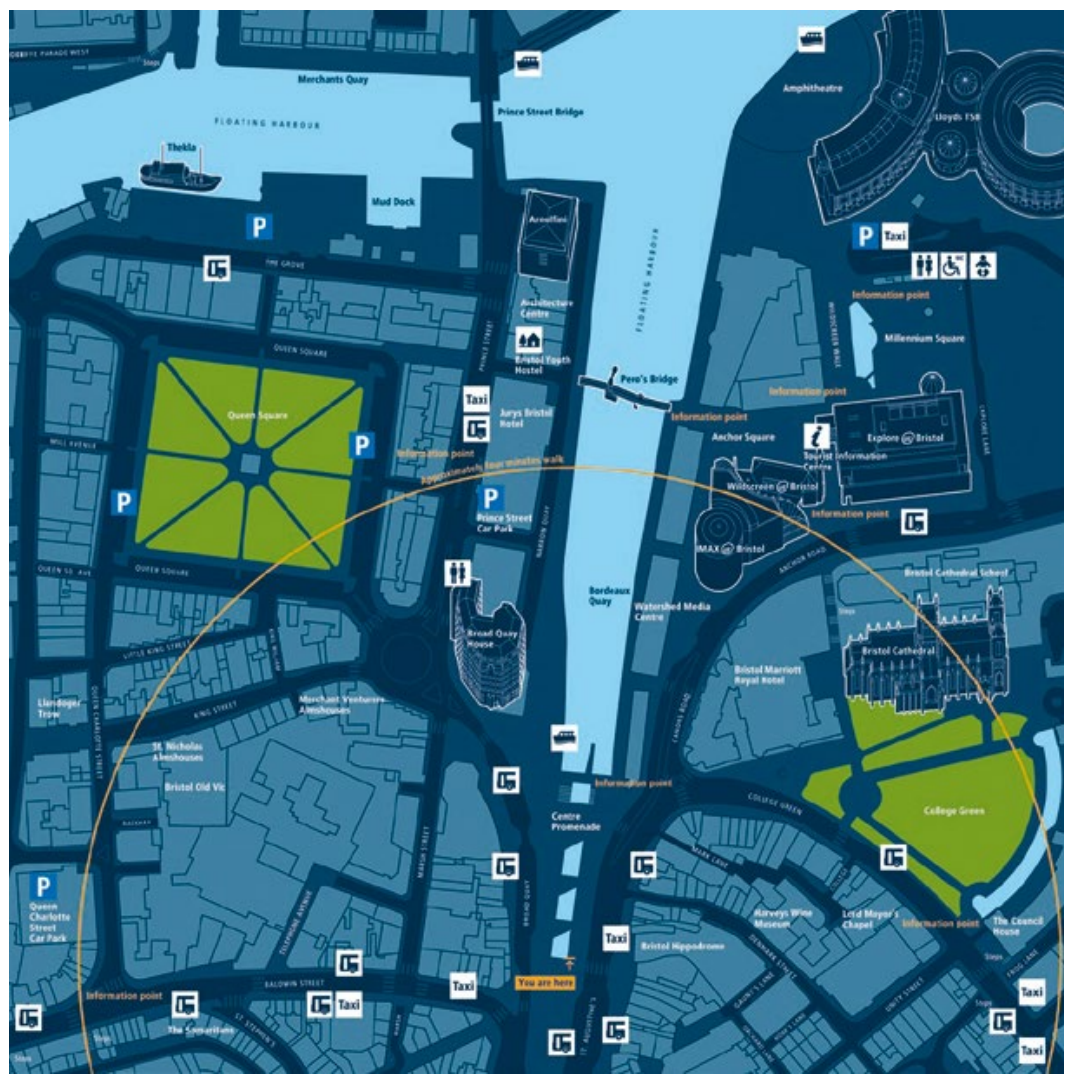

Language of Vision sind seine Thesen zur Visuellen Kommunikation zusamApplied Information Group London: «Legible City» mengefasst. Laut Kepes sollen topografische Merkmale als Gestaltungselemente betrachtet werden und für Geschlossenheit, Ordnung, Eindeutigkeit und Klarheit stehen.

Ausschlaggebend für das erfolgreiche Navigieren ist der Abgleich der Karte mit der physischen Umgebung. (1995), hier ein Ausschnitt von Bristol

Für den Abgleich gibt es zwei Strategien: Bei der Alltagsnavigation durch eine gewohnte Umgebung beruht der Vorgang auf den bisher gemachten Raumerfahrungen; bei der Raumnavigation anhand einer Karte stützt sich das Abstimmen auf die Karte. Für beide Navigationsformen ist das innere Bild zentral: Sei es dieses, das sich aus unseren Erfahrungen gebildet und einge- 
prägt hat, oder jenes das wir beim Studieren einer Karte verinnerlicht und gelernt haben. Beide Vorgänge funktionieren als Feedbackschleife, in dem "gespeicherte, räumliche Informationen zusammen mit daraus abgeleiteten Übersetzungsschemata in praktische Entscheidungen und Handlungen übersetzt werden". (Abend 2013: 53) Um überhaupt navigieren zu können, braucht es also die Erfahrung beziehungsweise die Fähigkeit, reale Gegebenheiten mit inneren Bildern, abzugleichen. In einer unbekannten Umgebung fällt dieser Abgleich oft schwer. Zur verbesserten Lesbarkeit von Städten und dem effizienteren Abgleich verwenden die Designer_innen von Applied Wayfinding einen 3-D-Effekt für Landmarken. Diese dreidimensionalen Darstellungen von Bauten kombinieren sie mit dem gewohnten Kartenstandard. Durch die Projektionsform /dreidimensionale Darstellung von Gebäuden) wird der Abgleich der Umgebung mit der schematischen Darstellung auf der Karte einfacher. Dieser bisher kaum eingesetzte 3-D-Effekt vereinfacht nicht nur den Navigationsprozess, sondern ermöglicht auch ein intensiveres Erlebnis von Stadtraum. Tim Fendley, dem Gründer und Creative Director von Applied Wayfinding, geht es unter anderem darum, Städte verständlicher zu machen und einem näher zu bringen. In ihrem Legible City Program, das speziell für Fussgänger entwickelt wurde, sieht er einen neuen Massstab für urbane Navigation. (Fendley 2014: 62-67) (Abb. 47) Zur Unterstützung des räumlichen Verständnisses und zum Abgleich der Karte mit der Umgebung werden häufig Landmarken verwendet. Wie wichtig Landmarken im Navigationsprozess sind, wird insbesondere in rein virtuellen Welten wie Google Earth erfahrbar. Durch das Fehlen von räumlichen Informationen ist die Orientierung erschwert, was einen oft verloren zurücklässt. Schon einfache Standortmarkierungen (Place Markers) jedoch unterstützen die Navigation und verschaffen Überblick.

In Verbindung mit Verortungstechnologien und standortbezogenen Diensten sind Navigationssysteme benutzerfreundlicher geworden. Durch die Komplexität und die grossen Datenmengen werden in naher Zukunft wahrscheinlich alle Konzepte in irgendeiner Form dynamisch, interaktiv und kontextbezogen sein. Bisweilen gibt es unter dem Begriff Digital Signage erste Ansätze interaktiver Orientierungs- und Leitsysteme. Solche Systeme kombinieren analoge Informationen im Raum mit digitalen Informationen auf dem Display (vgl. Schwanke-Seer 2013: 136). Wichtig ist dabei nach wie vor, dass räumliche Bezüge geschaffen werden, sei es durch Selbstlokalisierung oder über eine Kontextualisierung. Dadurch kann eine Desorientierung verhindert werden. Meines Erachtens birgt die Technologie von Augmented Reality ein grosses Potenzial für solch kom- 
binierte Systeme. Wie weiter oben herausgearbeitet wurde, kann mittels Augmented Reality die physische Umgebung mit virtuellen Informationen überlagert und erweitert werden. Damit kann sich besser auf die Umgebung konzentriert werden, die lesbarer und somit auch navigierbarer wird. Denn mit dem Einsatz und der Verwendung von Augmented Reality muss der Raum mental nicht mehr konstruiert, sondern kann in Echtzeit um grafische Elemente erweitert werden. Die Gründe für die Zurückhaltung bei der Entwicklung kombinierter Systeme dürfte zum einen im Entwicklungsstand liegen, zum anderen bei den Stakeholdern: Es liegen zu wenig brauchbare Referenzprojekte vor und Studien zu Kosten/Nutzen-Faktoren fehlen. Wie gross das Potenzial von Augmented Reality für die Navigation ist, wird bei der Gestaltung von HUDs für die Automobilindustrie sichtbar.

2018 präsentierte Google Maps auf der Entwicklerkonferenz Google I/O ein AR-Navigationstool für Fussgänger. Dabei verwendet Google Maps erstmals Augmented-Reality-Elemente für die Navigation. (Abb.48) Wie lange es bis zur Marktreife dauern wird, ist ungewiss. Die vorgestellte App überzeugt auf Designebene noch nicht, aber auf jeden Fall setzt sie einen neuen Standard im Design. Bereits älteren Datums ist die Zusammenarbeit der Johannes Kepler Universität Linz mit Siemens und dem Ars Electronica Futurlab an einem Navigationssystem, bei dem einzelne Komponenten des Autos in den Prozess miteinbezogen werden. Auf der Windschutzscheibe erscheinen Richtungspfeile, ähnlich wie

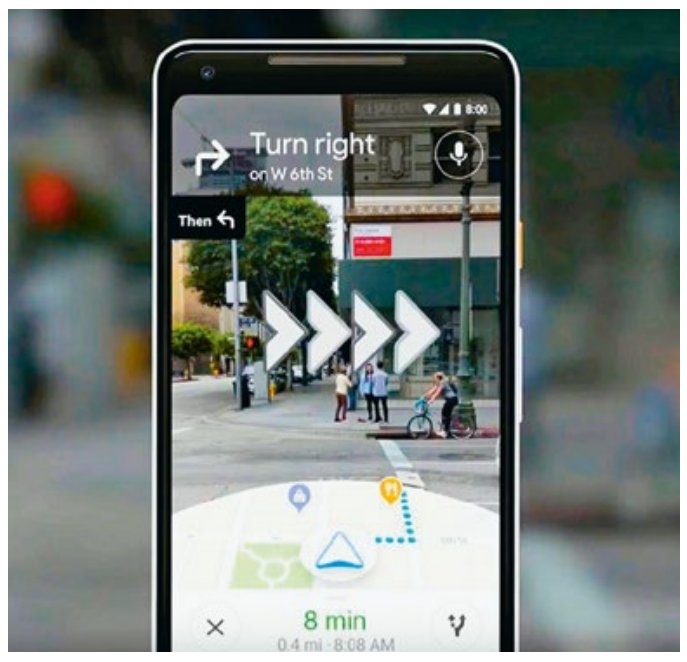

Ein Prototyp für ein Fussgänger-Leitsystem, das die Technologie von Augmented Reality nutzt 
bei Google Maps, denen zu folgen ist (vgl. Narzt et al. 2006, 2004). Gemäss den Forscher_innen haben Tests gezeigt, wie hilfreich ein solches Navigationssysteme sein kann, da herkömmliche Autonavigationssysteme oftmals zu abstrakt bleiben. Die Vorteile von AR-Navigationssystemen ist das immersive Erlebnis und damit eine bessere User Experience. Ein zukünftig wichtiger Punkt bei der Gestaltung von Augmented-Reality-Navigationssystemen wird der Übergang der virtuellen Welt in die physische Umgebung sein. In Bezug auf User Experience, Usability und Interaktivität wird eine einfache Bedienung sowie die optische Aufbereitung der Informationen zentral werden. Durch kontextspezifische Informationen kann sich die Information an den Nutzern orientieren und nicht umgekehrt, wie es bisher der Fall ist, also dass sich die Nutzer an der Information ausrichten müssen. Negativ auf Augmented-RealityNavigationssysteme wird sich ein schlechtes Design oder eine Informationsüberflutung auswirken.

\section{Weitere Navigationsformen - Routenwissen und Wegstrecken}

Für den Orientierungs- und Navigationsprozess ist die Unterscheidung zwischen Karten- und Routenwissen wichtig. Routenwissen ist insbesondere bei der Fortbewegung mit dem Auto bedeutsam, aber auch mit öffentlichen Verkehrsmitteln oder zu Fuss. Mit Routenwissen sind die Kenntnisse über den Weg beziehungsweise das Wissen, wie der Weg gefunden wird, gemeint. Das heisst die räumliche Repräsentation respektive die kognitive Karte, welche wir in uns abspeichern. Eine der ältesten heute bekannten Karten mit Routenwissen ist die aus dem spätrömischen Original über Kopien hervorgegangene "Tabula Peutingeriana». (Abb.49) Die Karte stellt das römische Reich inklusive Militärstationen und Distanzen von West nach Ost dar. Die Informationen auf der Karte sind linear aufgebaut. Die reale Entfernung ist somit nicht von Bedeutung, weshalb die Karte einer grossen Verzerrung unterliegt. Die Distanzangaben sind jeweils als Text angebracht. Nach heutigem Verständnis wäre die "Tabula Peutingeriana" vergleichbar mit einer modernen MichelinStrassenkarte, auf der Raststätten, Tankstellen und Distanzangaben eingezeichnet sind. Also ein Routenplaner mit Informationen zum gewählten Streckenverlauf.

Im Laufe der Zeit wurde die Wegstrecke mit Kartenelementen wie Massstäblichkeit oder Ausschnitt (aus einem Blattwerk oder 


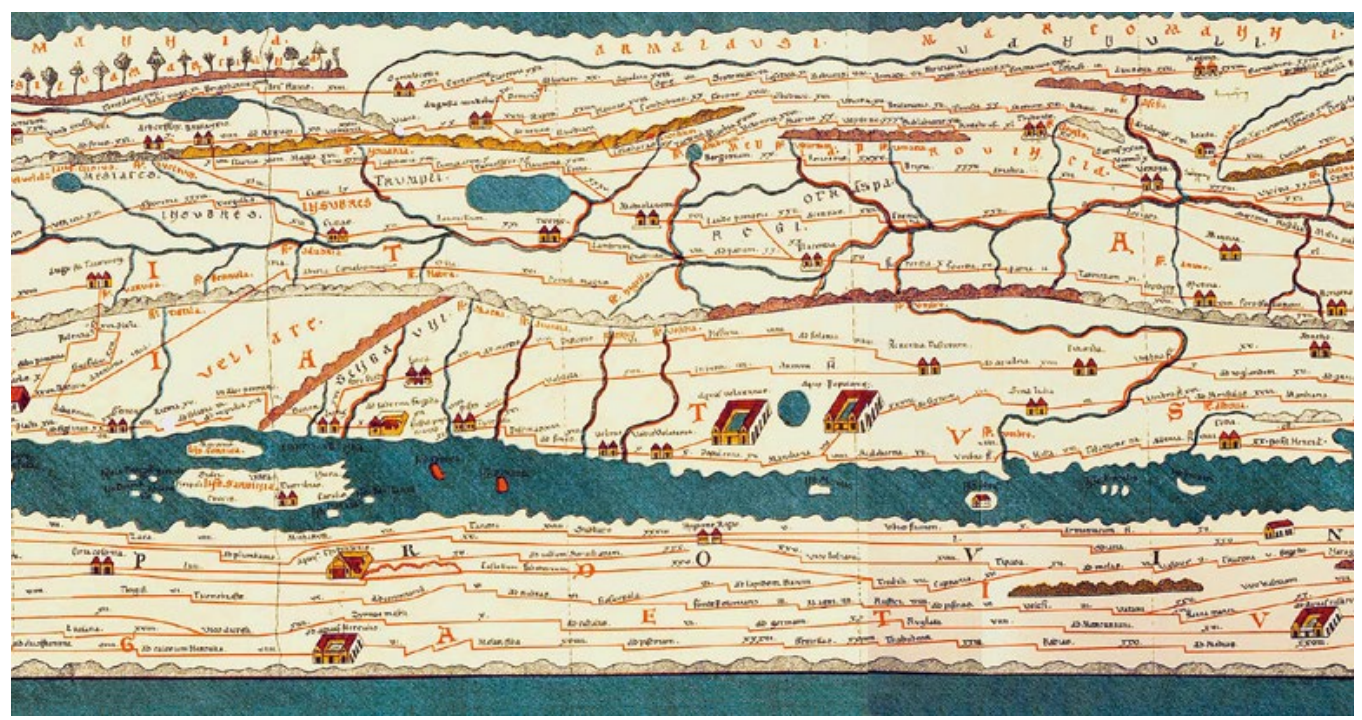

Peutinger'sche Tafel aus dem 12. Jahrhundert $(6,8 \times 0,34 \mathrm{~m})$, hier das dritte Segment (Detail) (Ausgabe von Konrad Miller, 1887, Ulrich Harsch Bibliotheca Augustana)

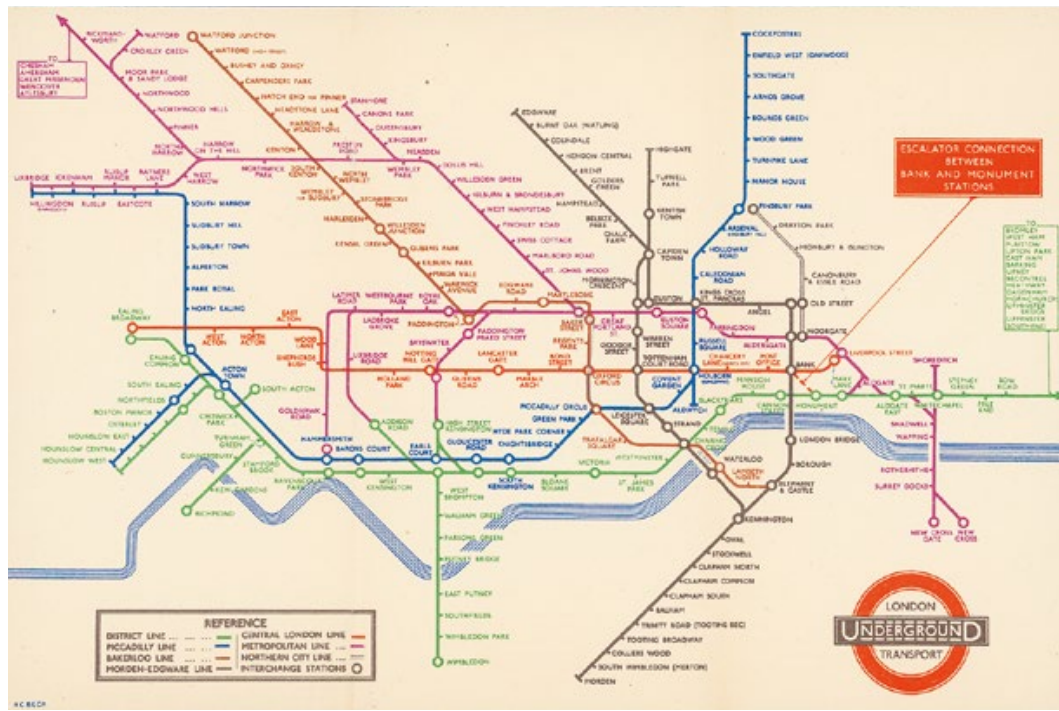

einer GIS-Datenbank) erweitert. Mit dem Aufkommen von VerortungstechRailway Map London Underground Transport (1935) von Harry Beck nologien sind Mischformen von Karten, Routenwissen und Handlungsanweisungen üblich geworden. Man denke etwa an ein Navigationsgerät für Autos, das ein Bild (eine Karte) aufweist, eine akustische Handlungsanweisung beinhaltet sowie Echtzeitda- 
ten zur aktuellen räumlichen Situation wie Verkehrsstau entlang des Wegs bereithält.

Wegstrecken sind stark vereinfachte beziehungsweise konstruierte Karten, bei denen die korrekte Raumrepräsentation der Funktion untergeordnet ist. Eine solche Karte wird zum Beispiel für öffentliche Transportmittel verwendet. Um diesen Kartentyp zu verstehen, braucht es kein Raumwissen: Ausreichend ist die Selbstlokalisierung (Wo befinde ich mich? Dies ist meist auf der Karte vermerkt) sowie das Kennen des Endziels (Wo möchte ich hin?). Der für die Londoner U-Bahn tätige britische Grafiker Harry Beck (1902 - 1974) machte sich 1933 über die bessere Lesbarkeit von U-Bahn-Plänen Gedanken. Um möglichst einfach und schnell an ein Ziel zu gelangen, ist bei einer U-Bahn-Fahrt nicht die geografische Realität, sondern die Raumbeziehung zwischen den einzelnen Stationen und Linien wichtig. Bei der Fahrt mit einer U-Bahn brauche ich mich also nur für das Ein- oder Aussteigen zu entscheiden, alles andere ist nicht relevant beziehungsweise beeinflussbar. Oder anders gesagt: Wenn ich von A nach B gelangen will, muss ich nur wissen, wie viele Stationen es bis zum Ziel sind, welche Linie ich nehmen muss und wo ich allenfalls umzusteigen habe. Daraus schlussfolgerte Beck, dass für die Zeichnung eines Streckenplans die Entfernung unwichtig, und nur die Abfolge und Beziehung des Wegenetzes bedeutsam sind. Dieser Gedanke übertrug er auf die Londoner U-Bahn-Karte und abstrahierte dadurch die bisher unübersichtliche Karte auf ein Minimum an geraden Linien und Punkten. (Abb. 50) Mit dieser neuartigen Darstellung gelang ihm ein Meilenstein in der Geschichte der Visuellen Kommunikation respektive im Informationsdesign. Durch die visuelle Reduzierung auf das Wesentliche prägte er einen neuen Standard, der bis heute dem Entwurf und der Gestaltung von Streckenplänen zugrunde liegt. 


\section{Limitationen und Potenziale digitaler Navigationssysteme}

Digitale Karten haben zweifelsfrei neue Formen der Navigation hervorgebracht sowie unsere Fortbewegung und unsere räumliche Orientierung verändert. Durch das bisher - zumindest im europäischen Raum seit der Mercator-Projektion - vorherrschende Ordnungsprinzip (Norden oben, Süden unten) bestand im Allgemeinen ein gewisser Konsens, in welche Richtung man sich bewegt. Auf digitalen Geräten sind jedoch Himmelsrichtungen nicht mehr zentral, das heisst, es wird immer nach vorne und oben navigiert. So ist weder eine Orientierung an der geografischen Ausrichtung noch an den topografischen Gegebenheiten möglich. Mit GPS wurde die Navigation durch Standortsuche, Routenplanung und digitalem Assistenten zwar einfacher, jedoch sind digitale Navigationssysteme auch flüchtig und unterliegen technischen Limitationen. Ein kleiner Bildschirm zum Beispiel reduziert die Orientierungshilfe, durch ständiges Scrollen geht der Überblick verloren und ein Vergrössern einzelner Teile macht einen Gesamtkontext schwierig. Die Nutzung technischer Geräte macht eigenständiges Navigieren überflüssig und daher kann auch kein Raumwissen angeeignet werden. Dies führt zu einem räumlich-kognitiven Verlust, was wiederum Auswirkungen auf den Hippocampus hat. Studien zeigen, dass mit Einsatz digitaler Navigationshilfen wie GPS-Geräte der Hippocampus zu schrumpfen beginnt. Ob der Hippocampus wirklich schrumpft, ist in der Wissenschaft aber weiterhin umstritten. Es konnte jedoch nachgewiesen werden, dass der Hippocampus während der Ausbildung angehender Taxifahrer wächst (vgl.Carr 2010: $326 \mathrm{ff}$.). Einig sind sich Wissenschaftler_innen darin, dass beim alleinigen Gebrauch von GPS-Geräten räumliche Bezüge nicht mehr erlernt werden können. Die kognitive Fähigkeit, sich im Raum zu orientieren und räumliche Beziehungen herzustellen, wird nicht ausgebildet, ähnlich wie bei einer ausschliesslichen Fortbewegung im Auto. Denn Auswahl und Komplexitätsreduktion der Route werden an eine Software beziehungsweise an Künstliche Intelligenz delegiert. Das eigene räumliche Denken und Verhalten werden durch einen Algorithmus beeinflusst. Eine Studie des University College in London brachte zu Tage, dass im Wettstreit mit Künstlicher Intelligenz Londons Taxifahrer noch gegen das Navigationsgerät gewinnen. Die Gründe sind einerseits in einer anspruchsvollen mehrjährigen Ausbildung und Prüfung zu finden, andererseits in der aktuell noch technischen Limitation der Geräte. Auch kann das GPS-Signal nicht überall (gut) empfangen 
werden. Das eingesetzte Kartenmaterial ist zudem teilweise veraltet und aktuelle Gegebenheiten und Beeinträchtigungen wie Baustellen und Quartierstrassen werden nicht berücksichtigt etc.

Neue geospatiale Praktiken wie die Verwendung von Satellitenbildern haben vermehrt zu einer Praxis des Kartenschauens geführt. Dazu gehört auch das Geobrowsing, das nur in einem virtuellen Umfeld wie beispielsweise Google Earth stattfinden kann.

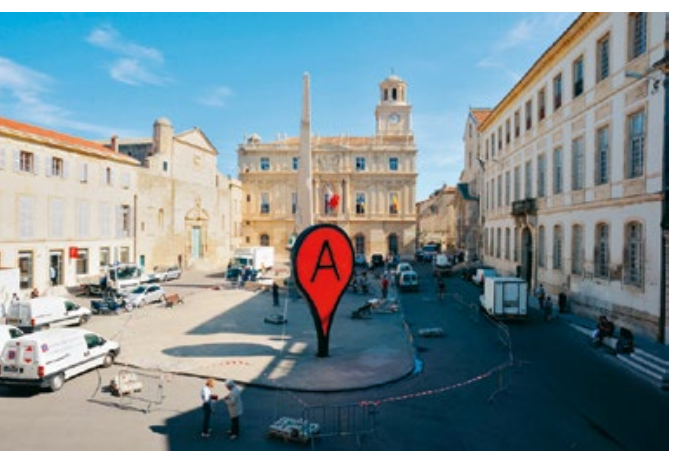

Abraham Bartholl: «Map» (2006 - 2013). Die Skulptur «Map» (2011) auf dem Marktplatz von Arles, Frankreich, während der Les Rencontres de la Photographie d'Arles

Hier wird die Navigation auf der Computeroberfläche zum Ersatz für die Bewegung im realen Raum. Die Wahrnehmung der Welt beschränkt sich auf einen Bildschirm und dieser wird zugleich zum Ausgangs- und Endpunkt der Reise. Im Gegensatz zum Flanieren mit einer Karte im Raum, bei welchem auf eine durch den Kartenurheber getroffene Vorauswahl (Generalisierung und Abstraktion) zurückgegriffen wird, kann beim Geobrowsing eine eigene Auswahl getroffen werden. Der Nutzer kann gleichzeitig sehen (Karte) und zumindest simuliert - gehen (Wegstrecke). Darüber hinaus kann er zwischen einer 2-D- und einer 3-D-Ansicht hin und her wechseln. Durch das gleichzeitige Gehen und Sehen kann er vor dem Bildschirm die Perspektive eines Voyeurs oder Flaneurs einnehmen, ohne die Position zu verändern. Nach de Certeau braucht es zur Ausbildung der Raumerfahrung jedoch die Bewegung beziehungsweise das Bewegen durch den Raum, wodurch der Raum vermessen, markiert und verortet wird: "Die Erzeugung eines Raumes scheint immer durch Bewegung bedingt $z u$ sein, die ihn mit einer Geschichte ver- 
bindet.”(de Certeau 1988: 219) Eine rein virtuelle Raumerfahrung dagegen entkörperlicht. Wohl auch deshalb gleichen die Strategien, die beim virtuellen Navigieren eingesetzt werden, derjenigen einer analogen Navigation. Mittels Standort-Marker können besuchte oder zu besuchende Standorte markiert und dadurch einfach wiedergefunden werden. Der deutsche Künstler Abraham Bartholl thematisiert in seinem Werk "Map» (2006-2013) dieses Markieren auf digitalen Plattformen und übersetzt den Prozess in raum-greifende Skulpturen. (Abb. 51)

Mit der Verbreitung von Verortungstechnologien wie GPS setzt sich zunehmend ein navigationsorientierter Gebrauch der Karte durch. Unter navigationsorientiert ist eine Kette von Handlungsanweisungen zu verstehen. Bei diesem Navigationstyp ist die grafische Erscheinung sekundär. Wichtig sind Start- und Endpunkte, akustische Anweisungen sowie Richtungsangaben während des Navigationsprozesses. Durch das veränderte Orientierungsverhalten schauen wir immer weniger auf die Karte - anfänglich beim Autofahren, mittlerweile auch beim Stadtrundgang - und lassen uns von einem technischen Gerät beziehungsweise von einer Stimme führen. Diese Navigationsform lehnt sich an Handlungsanweisungen an, in welchen die Ausführung (Fortbewegung) wichtiger wird als das Verstehen (Sehen). Ein Nachteil solcher Navigationssysteme ist, dass sie akustische Anweisungen nutzen anstelle von bildlichen Elementen. Die akustischen Angaben bleiben dabei jedoch vielfach zu abstrakt. Eine nach dem Weg suchende Person, die bisher daran gewöhnt war, sich im Raum und an Landmarken zu orientieren, wird die räumlichen Bezüge vermissen. Die Aufforderungen wie nach 200 Metern links abbiegen, sind oftmals zu unspezifisch, da es an Referenzpunkten zur Orientierung und an der Bestätigung fehlt. Der Einsatz digitaler Navigationshilfen hat somit auch Auswirkungen auf unser Orientierungs- und Navigationsverhalten. Die Umgebung bietet keine lesbare Führung mehr, wie sie von Lynch oder Kepes herausgearbeitet worden ist. Hier gibt es ein grosses Potenzial für die Designwissenschaft bezüglich der Forschung nach neuen Standards. 



\section{Die Praxis des Erzählens}

Mit der kritischen Kartografie und den immer weiterschreitenden technologischen Möglichkeiten werden topografische Karten seit dem digitalen Spatial Turn um subjektive Kartierungsformen erweitert. Innere beziehungsweise kognitive Karten unterscheiden sich grundlegend von topografischen. Sie zielen nicht darauf $a b$, die geografische Realität möglichst genau wiederzugeben, sondern kartieren einen inneren Gemütszustand wie zum Beispiel Gefühle. Sie können, müssen aber nicht zwingend eine geografische Karte zur Grundlage haben. Mithilfe von ortsbasierten sozialen Netzwerken und narrativen Methoden können persönliche Karten entstehen. Dabei wird insbesondere auf bewährte Techniken wie Tracking (Aufnehmen einer Tätigkeit), 
Tracing (Visualisieren einer Tätigkeit) sowie Storytelling und Interaktion zurückgegriffen, die über das eigene Mobilgerät und mithilfe neuster digitaler Dienste selbst ausgeführt werden kann. Dies erlaubt Nutzern standardisierte Karten mit privaten Inhalten $\mathrm{zu}$ erstellen.

Das Produzieren und Repräsentieren der Welt im Internet ist technisch seit Web 2.0 und mit Mashups möglich. Die Kartenherstellung wurde dadurch zu einem Amateur-Feld, was zu einer Verschiebung der Hierarchie von Produktion und Rezeption führt. Alle können Teil des Geo-Webs werden und Karten oder räumliche Daten produzieren, teilen und verbreiten. Mit diesem Trend, ausgelöst durch eine Kultur eines ubiquitären Computings, entstehen Karten, die nicht ausschliesslich zur räumlichen Orientierung bestimmt 
sind. Vielmehr produzieren diese Karten und Kartografien neue Räume, sei es durch Teilnahme, Inszenierung oder Aneignung. Dadurch verändert sich der Gebrauch der Karte von einem Medium, das Zusammenhänge verräumlicht, zu etwas, das räumliche Information in einen sozialen Kontext stellt und eine Kultur des Kartierens begründet. Zugleich entstehen neue Interaktionsformen $z$ wischen den vernetzten Nutzern und dem sie umgebenden Raum. 


\section{Kartierungen des eigenen und äusseren Umfelds}

Die Raumkonzepte der Situationistischen Internationale (1957) rund um Guy Debord sind unter anderem beeinflusst von dem Raumtheoretiker Henri Lefebvre. Kartierungen des eigenen und äusseren Umfelds lassen sich auf Methoden und Strategien der Situationisten und der surrealistischen Bewegungen zurückführen. Die Situationisten, die sich um den französischen Künstler und Kapitalismuskritiker Guy Debord (1931-1994) gruppierten, bauten in den 1950er-Jahren ihr Konzept der Dérive auf (frz.= vom Kurs abkommen, abweichen oder ableiten). Das Konzept schlägt eine alternative Bewegungsform durch den Stadt-Raum vor: Anstatt auf dem effektivsten von A nach B zu gelangen, sprich dem kürzesten oder schnellsten Weg, folgt man den Spuren eines alternativen Bewegungsmusters. Dieses leitet sich entweder von einem Schema ab (wie z.B. erste rechts, zweite links, siehe hierzu auch Perec 2013) oder basiert auf dekontextualisierten Plänen wie einem Schnittmuster, das als Stadtplan benutzt wird. Das Konzept lehnt sich an den Begriff des Flaneurs an, der auf Walter Benjamin und die Surrealisten zurückgeht. Der Flaneur ist ein auf der Suche nach Zerstreuung - durch die damals aufkommenden Passagen der 1930er-Jahren - ziellos treibender Müssiggänger. Unter jeglicher Missachtung von Zeit und Geschwindigkeit führt der Flaneur eine Schildkröte als Sinnbild für die Langsamkeit mit sich. Davon inspiriert, liessen sich die Situationisten ebenfalls planlos durch die Stadt treiben und folgten dabei alternativen Routen und Wegen, wie etwa einer Statistik oder einem Aktienkurs. Ihr Ziel war die totale Verschmelzung von urbanem Raum, Leben und Kunst. Die Stadt soll Einfluss auf die Innenwelt ausüben, auf Emotionen, Handlungen und das Verhalten. Die Raumaneignung basiert dabei auf eigenen Erfahrungen und wird nicht durch vorgegebene Repräsentationsmuster beeinflusst. Dadurch werden zufällige Situationen geschaffen, Momente, die gegen eine totale Effizienz rebellieren, die mit Ordnung und Gewohnheiten brechen (vgl. auch Debord 1996). Die Situationisten durchwanderten nicht nur urbane, sondern auch mentale Räume, indem sie sich in andere Situationen versetzten (Cognitive Map). Für den "Guide Psychogéographique de Paris» untersuchte Debord beispielsweise die Wirkung der Stadt auf die Wahrnehmung (vgl. Debord 1996). (Abb. 52) Die zerschnittenen Strassen symbolisieren die unterbrochenen Räume, welche bei der Benutzung einer Metro oder einer Strasse entstehen. Durch die Nutzung des Verkehrsmittels geht jeglicher Gesamtzusammenhang der Stadt verloren. 


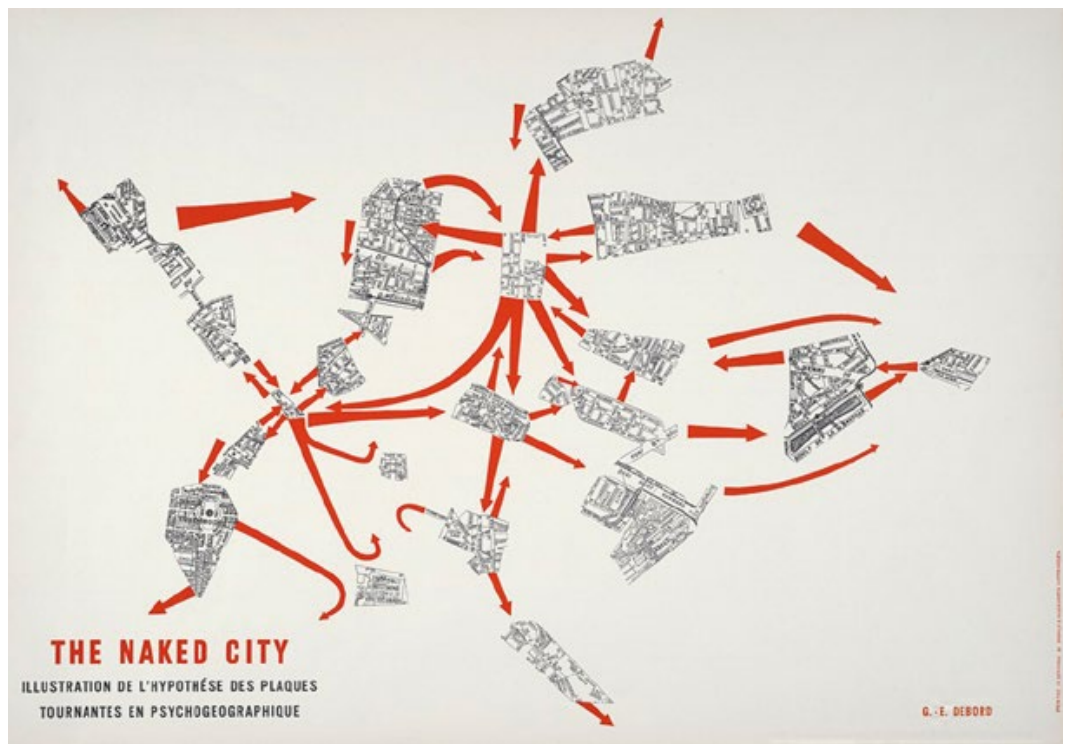

In der Überwindung des Effizienzgedankens liegt auch die politische Guy Debord und Asger Jorn: «The Naked City» (1957) Sprengkraft einer Dérive. Globalisierungsgegner und Urban Guerillas wie zum Beispiel das holländische Kollektiv Social Fiction nutzt die Dérive, um ein Netzwerk neuer Pfade im kapitalistisch organisierten Stadt-Raum anzulegen. Und die beiden Performer und Tänzer Jochen Roller und Martin Nachbar erkunden in Anlehnung an die Dérive die Möglichkeit für die Performativen Künste. Ginge es im Tanz um Effizienz, also um die ökonomische Überwindung der Distanz zwischen Punkt A und Punkt B, würde sich das Bewegungsvokabular eines Tanzstückes auf wenig mehr als das Gehen reduzieren. In Form einer Dekonstruktion erleben die Performer im Tanz die Nicht-Effizienz der Bewegung einer Dérive: nämlich auf indirekte Weise zwei Punkte im Raum miteinander zu verbinden. Um die Anatomie von Städten zu untersuchen, begeben sie sich auf eine räumlich-choreografische Erkundungstour durch europäische Städte. 


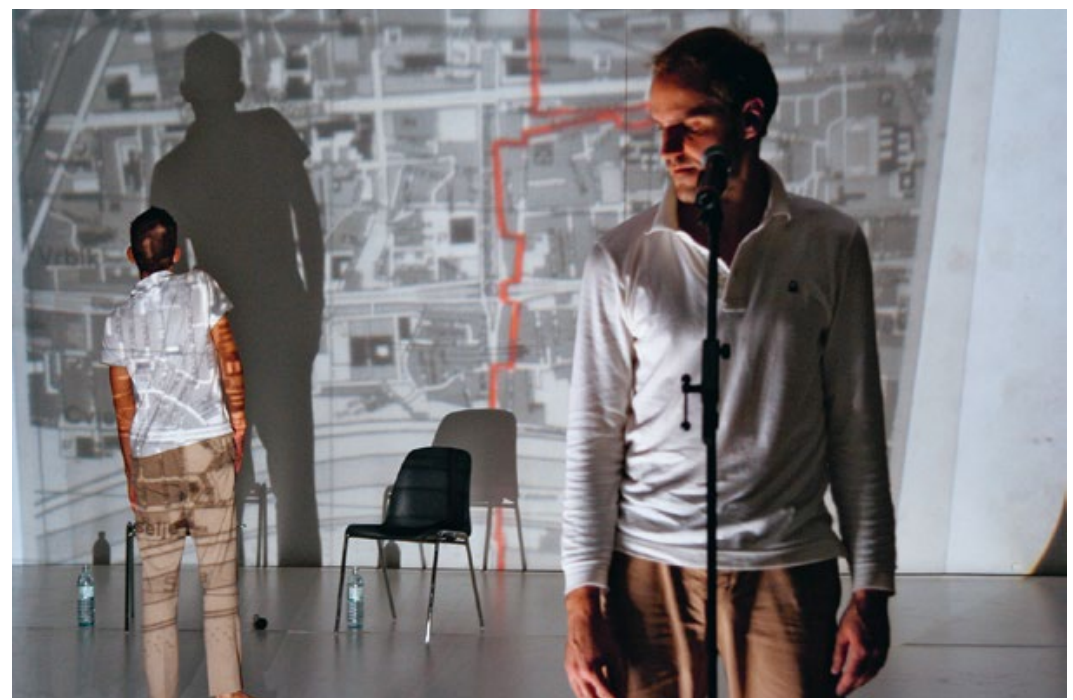

Auf jeder Exkursion erstellen sie Karten: Strassenverläufe werden mit GeJochen Roller und Martin Nachbar: «Nemonic Nonstop» (2003) danken assoziiert, Begegnungen simultan verzeichnet und Beobachtungen darauf vermerkt. Durch das Übereinanderschichten der mitgebrachten Karten formen sich neue Wege, die die beiden Tänzer bei der Arbeit anleiten. In der daraus hervorgegangenen Tanzperformance “Nemonic Nonstop" (2003) werden die Wege mittels Folien in den Bühnenraum projiziert. (Abb.53) Die beiden Tänzer folgen in ihren Bewegungen den Wegen und stellen sie durch gesprochene Texte in den Kontext der jeweiligen Stadt und der dort gemachten Erfahrung. Durch das Übereinanderlegen des kartografischen Materials transformieren sie die Schichten: Projektionen, Text und Bewegung ergeben eine ephemere Karte, die nur durch die Aufführung entsteht und danach wieder verschwindet. Die Bühne wird zu einem kinetischen Logbuch, durch das die Performer tanzend als Kartografen navigieren. So wie in Jochen Rollers Jugend Westberlin auf den Karten der DDR nur als weisse konturlose Fläche 
jenseits der Stadtgrenze von Berlin (Ost) existierte, alterniert das System die Strassennamen auf den Stadtplänen. In Brüssel zum Beispiel, je nachdem ob man die Karte eines flämischen oder eines wallonischen Verlags in der Hand hält.

Topografische Karten vermessen Städte nach vermeintlich objektiven geografischen Kriterien. Durch die Karte wird immer auch eine Idee des Kartografen vom Stadt-Raum repräsentiert: Der Vorgang des Kartografierens wird zur Beschreibung des Raums. Eine Vordenkerin, die sich mit diesen Tendenzen beschäftigte, war Jane Jacobs (1916-2006), die in The Death and Life of Great American Cities (1961) mittels räumlicher Bezüge auf die soziale Verarmung in amerikanischen Grossstädten aufmerksam machte. Jacobs ging es um individuelle Perspektiven, um den gelebten und erfahrenen Raum, um Bezüge zwischen Person und Umgebung. Heute können ähnliche Tendenzen unter dem Begriff Cultural Mapping gefasst werden. Unter Cultural Mapping werden innerhalb eines urbanen Raums, einer Stadt vielfältige Konzepte zur Vernetzung von Mobilität, Kommunikation und lokativen Medien verstanden. Hierfür zeichnet sich ein Paradigmenwechsel verantwortlich, der den Raum aus seinem primären architektonischen Grundverständnis hin zu einem sozialen Diskurs über den Raum geführt hat (vgl. Hemmersam et al. 2015: 171) Schon in frühen architektonischen Studien wie denjenigen von Jacobs waren lokale, ortsspezifische und individuelle Erfahrungen und Wahrnehmungen prägend. Seit der Entstehung von GIS sind räumliche beziehungsweise verräumlichte Daten ubiquitär geworden. Solche Daten geben Aufschluss über visuelle Muster. Ein beliebtes Untersuchungsgebiet für räumliche Analysen ist, wie durch Social Media und mittels vernetzter Kommunikation Städte beziehungsweise städtisches Gefüge wahrgenommen, geformt und erfahrbar werden. 


\section{Locative Media}

Der Begriff Lokative Medien (Locative Media) wurde erstmals im Kunstkontext geprägt und meint heute ortsunabhängige beziehungsweise ortsbezogene digitale Medien (obwohl die Medien ortsunabhängig sind, bleibt der Inhalt ortsgebunden). Deshalb ist eine Unterscheidung von Ort und Medien wichtig geworden. «Lokative Medien initiieren eine Neucodierung des Orts als clocality, die sich vom Kartographischen und Abstrakten weg und hin zum Sozialen und Vernetzen bewegt.) (Buschauer \& Willis 2013: 16) Mit dem Aufkommen von Ortsmedien (allen voran Navigationsgeräte) sind auch kommerzielle Dienste entstanden. Diese sind insbesondere in den sozialen Netzwerken und mit standortbezogenen Diensten auszumachen, wie beispielsweise Foursquare. (Abb.54) Foursquare ist eine beliebte Plattform, auf der Nutzer Standortinformationen miteinander teilen können. Die in den letzten Jahren durch diese Dienste entstandenen Karten dienen kaum der Navigation, vielmehr produzieren sie neuen Raum, indem sie beispielsweise gesellschaftliche, politische, soziale oder persönliche Zusammenhänge visualisieren. "Hypertrending" von Foursquare ist eine Echtzeitkarte, auf welcher sichtbar wird, wie sich die Nutzer in der Stadt verteilen. Jeder farbige Punkt repräsentiert einen Standort, die Grösse des Punkts entspricht der Anzahl der Nutzer (basierend auf der Anzahl der Telefone). Zur Herstellung solcher Karten wird auf ortsbasierte Dienste und Kartentools zurückgegriffen. Künftig soll auch die vierte Dimension -

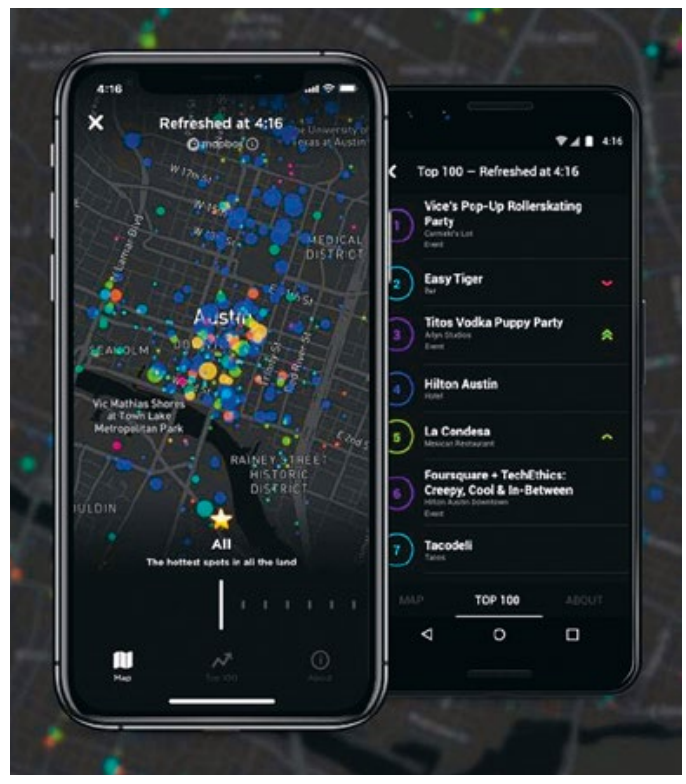

Die Echtzeitkarte «Hypertrending» von Foursquare, hier die Stadt Austin 
Zeit - integriert werden. Im Zuge dieses Wandels sind aus Nutzern nicht nur Konsumenten_innen, sondern auch Produzenten_innen geworden. Jedermann kann heute Karten produzieren, teilen und damit eine subjektive Sicht auf die Welt präsentieren. Die Produzenten_innen verfolgen dabei Strategien und Methoden wie Partizipation, Aneignung oder Inszenierung eines Orts, eines Raums. Ein wichtiger Aspekt ist, dass die Karten den physischen Ort mit persönlichen Notizen, Geschichten und Bildern (über-|beschreiben und dadurch den bestehenden Raum erweitern oder gar einen neuen Raum produzieren - das Geo-Web. Das durch Nutzer generierte Raumwissen macht einen Ort mehrschichtig und kann ihn für einzelne Benutzer wertvoller machen. Wichtig ist aber auch, sich bewusst zu sein, dass mit der Nutzung des Dienstes Standortdaten gesammelt werden. Dadurch lassen sich Gewohnheiten herausfiltern und zum Beispiel für Werbung vermarkten.

Bevor lokative Medien kommerziell vermarktet worden sind, wurden sie von Künstler_innen verwendet. Pionierinnen, die bereits in den 1990er-Jahren GPS als Kunstform einsetzten, sind zum Beispiel die Künstler_innen Laura Kurgan, Teri Rueb und Masaki Fujihata. Heute werden solche Strömungen unter Locative Arts gefasst (vgl. Hemment 2006) und meinen die Verbindung von mobilen Geräten mit Ortsbestimmungen in einem Kunstkontext. Im Wunsch der Erforschung seines Innenlebens und des Stadtraums finden sich Parallelen zu analogen Konzepten der Situationisten oder zu architektonischen Studien wie diejenigen von Jacobs. Die Praktiken und Methoden können technisch (Laura Kurgan), subjektiv (Jeremy Wood) oder narrativ (Janet Cardiff) sein und "stehen [...] zwischen einer technischen Raumordnung und alternativen Epistemologien des Raumes " (Buschauer \& Willis 2013: 18). Oftmals beinhalten die Strategien und Methoden alternative Kartierungsformen, welche Bewegungen im Raum nachverfolgen, messen oder aufzeichnen. Der britische Künstler Jeremy Wood hat beispielsweise über neun Jahre für sein Werk “My Ghost» (2009) mittels eines GPS-Empfängers seinen Weg durch London aufgezeichnet. (Abb. 55) Wood selbst nennt seine Arbeit “GPS-drawings»: “[V]isual journals that document a personal cartography. " Durch die Möglichkeit, narrative Visualisierung und Technologien zu verbinden, verlagert sich der Zugang zur Kartografie zunehmend zu einem des Kartierens - zur Vermessung der äusseren und inneren Welt. Die Kulturtechnik des Kartierens wurde durch die neuen Medientechnologien und -möglichkeiten zu einem Massenphänomen und einer gesellschaftlichen Praxis. Dabei sind auch Aspekte einer kritischen Kartografie wichtig, die die Karte als Medium der Machtquelle hinterfragen, neu definieren oder 


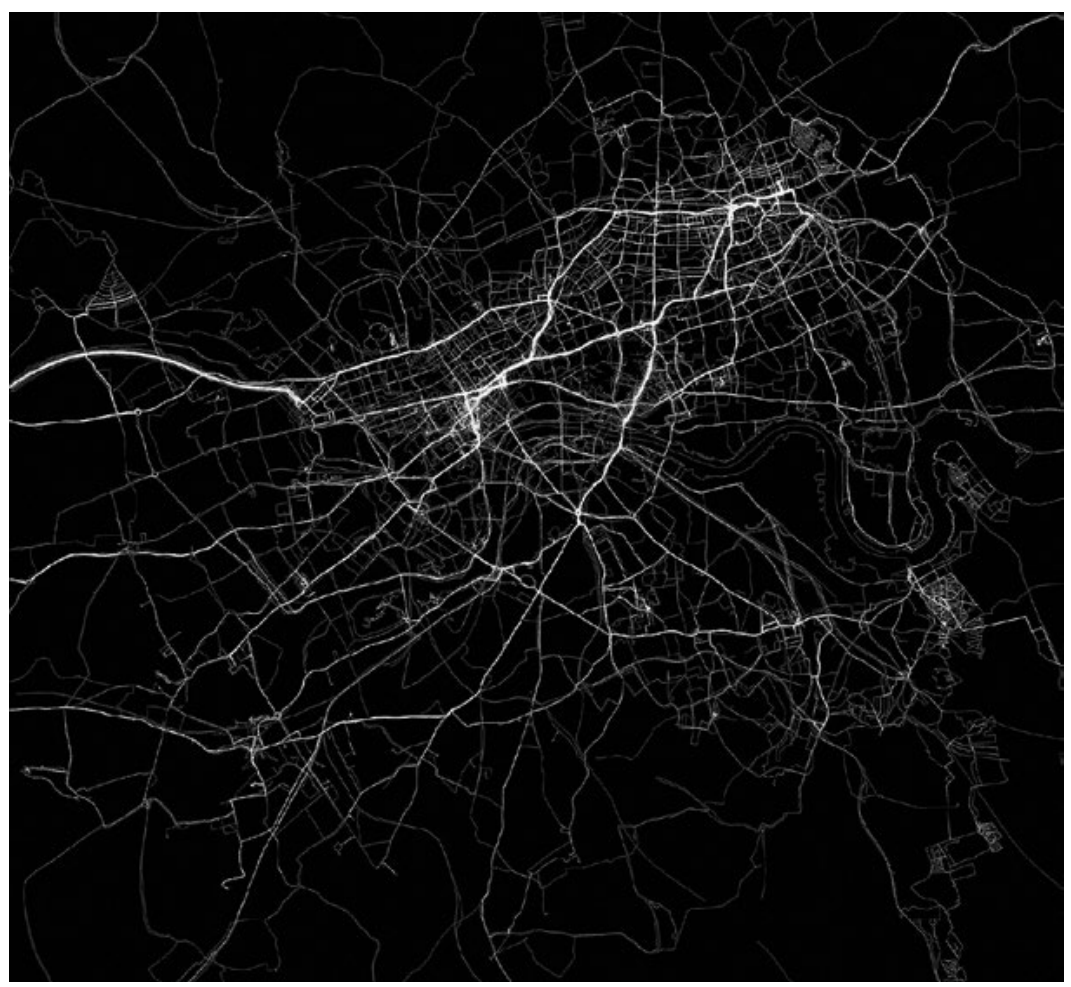

alternative Produktionsformen bezie- Jeremy Wood: «My Ghost» (2009)

hungsweise subversiv-künstlerische Praxen ermöglichen.

Die Kunstform Locative Arts hat sich programmatisch aus der ortsspezi$\mathbf{N}$ fischen Kunst (Site-specific art) beziehungsweise der skulpturalen Praxis der Minimal Art entwickelt. Ortsspezifische Werke können temporäre oder feste Interventionen sein, die sich bewusst mit der Geschichte und der physischen oder materiellen Präsenz eines Orts befassen. In der bildenden Kunst spricht man von Ortsspezifität, wenn der Raumbezug eines Werks thematisiert wird. Unter dem Einfluss der technologischen Entwicklungen haben sich diese Tendenzen um den Raumbezug mit einer technologischen Komponente erweitert. In den 1970er-Jahren wurde die ortsspezifische Kunst von der Land Art und der Konzeptkunst aufgegriffen, 
um die Beziehung zwischen Werk und Kontext zu erweitern.

Das Gehen als Kunstform etabliert haben die beiden britischen Künstler Richard Long und Hamish Fulton. Beide nutzen das Wandern als Kunstmedium und werden auch als Walking Artists bezeichnet. Richard Longs Arbeiten geben detailliert Auskunft über seine zurückgelegten Wege, auf welchen er mit den vorhandenen Materialien ortsspezifische Eingriffe zurücklässt. Zu seinen berühmtesten Werken gehört "A Line Made by Walking" (1967). Um die erwanderten und durch die Begehung veränderten Landschaften zu dokumentieren, nutzt Long die Medien Fotografie, Text und Karte. Hamish Fulton verarbeitet in seinem Werk ein- bis mehrtägige Wanderungen. Auf diesen Touren erfasst er seinen emotionalen und physischen Zustand die persönliche Erfahrung, die er jeweils in der Landschaft macht, und die Stimmung, die die Landschaft in ihm auszulösen vermag. Dabei schreibt er akribisch die Länge und Dauer, die klimatischen Bedingungen sowie seine gesammelten Beobachtungen auf. (Abb.56) Beide Künstler nutzen zur Darstellung ihrer Raumaneignung die Typografie und grafische Elemente, um detailliert über die zurückgelegten Wege Auskunft zu geben. 


\section{Erzählung im (vernetzten) Raum}

In Zeiten von Social Media und ortsbasierten Diensten gehört das Storytelling, das mit der Verbreitung von webbasierten Medien Einzug hielt, zu einem schnell expandierenden Bereich. Partizipative, nicht-lineare Erzählungen im (vernetzten) Raum, die ihre Inhalte und Bedeutung aus der Interaktion mit den Nutzern und der Umgebung entwickeln, sind beliebt. Storytelling ist eine Möglichkeit, dem passiven Medienkonsum zu entkommen, und reagiert auf eine zunehmende Lesemüdigkeit linearer, statischer Texte. Zur Wissensvermittlung, zur Strukturierung und zum Informationszuwachs können zur Veranschaulichung narrative Strategien verwendet werden. Die mobil vernetzte Welt sowie lokative Medientechnologien ermöglichen eine völlig neue Form, Geschichten zu erzählen. Beispielsweise können durch Interkation neue Handlungsstränge entstehen und der Plot kann mit der Umgebung oder dem Standort des Lesers verschmelzen. "The story space is now both the physical world and its augmentation.” (Hight 2013: 246) Der Nutzer wird Teil der Geschichte und kann auf deren Verlauf und Ausgang einwirken. Auch für de Certeau spielt für die Konstitution von Räumen die Erzählung eine wichtige Rolle. Durch ihre "performative Kraft (sie macht, was sie sagt) [...] schafft sie Räume. Umgekehrt gilt, dort, wo die Erzählungen verschwinden (oder zum musealen Gegenstand verkommen), kommt es zu einem Raumverlust. "(de Certeau 1988: 228) Das heisst, dass Räume eben nicht alleine durch ihr physisches Dasein definiert sind, sondern in ihrer Inszenierung entstehen. Die Erzählung ist neben der Bewegung - die es braucht, um überhaupt Räume zu schaffen - die treibende Kraft zur Entstehung von Räumen, die der Vermittlung von Geschichte und dem Wissenszuwachs dienen. Oder in anderen Worten: Erzählungen vermögen einen Ort in einen Raum zu verwandeln. Durch verschiedene Erzählstränge wird das Hin- und Hergehen in der Zeit und im Raum möglich. Bereits die historische Theater-Avantgarde experimentierte mit Konstruktion und Realität, indem sie an Originalschauplätzen Theater schuf. Das zeitgenössische Theaterkollektiv Rimini Protokoll nutzt ortsspezifische Erzählstrategien in seinen Theaterinszenierungen. In «50 Aktenkilometer - ein begehbares Stasi-Hörspiel» (2011) verwendet das Kollektiv den realen (Stadt-)Raum Berlin als Bühne. Die Wirklichkeit wird darin Teil der Aufführung. Fiktives überlagert sich mit Realem, wodurch ein Raum von verschiedenen Realitäten entsteht.

Das Projekt “34 North 118 West» (2002) der amerikanischen Medienkünstler Jeff Knowlton, Naomi Spellman und Jeremy Hight wird als eine der ersten lokativen Medienerzählung bezeichnet. 
(Abb.57) Auf dem Areal eines alten Eisenbahndepots wird an ausgewählten Orten die Geschichte der EisenbahnIndustrie in Los Angeles offengelegt. Mittels einer interaktiven Karte, die jeweils Auskunft zum eigenen Standort gibt, wird die Gegenwart mit der Vergangenheit verwoben, was den Ort zu einem ortsspezifischen Erzählraum macht. Die Standorte, an welchen die Geschichten spielen respektive abge-

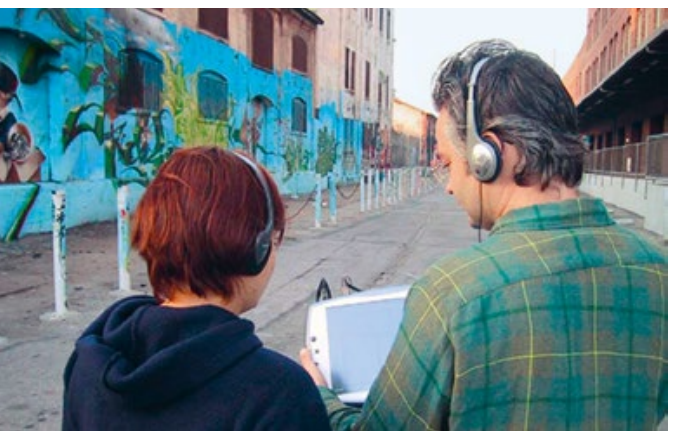

Jeff Knowlton, Naomi Spellman und Jeremy Hight: «34 North 118 West» (2002)

spielt werden, sind auf einer Karte verzeichnet, der Zugang erfolgt über Computer, Kopfhörer und GPS-Signal. Durch das Vor- beziehungsweise ImOrt-Sein wird eine Erfahrung möglich, die sich von kontextlosen Museumsvermittlungen oder rein medialen Erlebniswelten wie Virtual-Reality-Räumen unterscheidet. "Locative media and locative narrative is instead a mixed reality space; one is active in both the physical word [sic!] and the technologically aided/added annotations and augmentation in real time." (Hight 2013: 250) Das Beispiel zeigt exemplarisch, wie Geschichte mit einem Orte verknüpft werden kann und wie Erzählungen von der Zeit losgelöst stattfinden können. Da Geschichte immer an einem Ort stattfindet (history takes place), plädiert auch der Historiker Karl Schlögel für eine Verräumlichung des Geschichtsdenkens. Darunter versteht Schlögel, die Geschichte nicht im Buch nachzulesen, sondern am Ort (also räumlich) zu erleben (vgl. Schlögel 2011). Eine reine Informationsebene reicht eben oft nicht aus, da Informationen eine Übersetzung ins Anschauliche benötigen. Das heisst, Informationen sollten inszeniert sein, können narrativ werden. Die Erhaltung 
der Vielfalt eines Orts, die durch das kulturelle Gedächtnis und die Einschreibung der Geschichte in einen Ort überhaupt erst möglich wird, ist ein wichtiges Anliegen bezüglich der Archivierung von Geschichte. Die Fähigkeit, Geschichte in einer nachgenutzten Umgebung zu erleben, bietet die Möglichkeit, Architektur und Kultur, Bräuche und Traditionen, alte Strassen oder fehlende Gebäude zu entdecken und das Verständnis für die Vergangenheit zu erweitern.

Die Navigation ist jeweils ein elementarer Bestandteil einer Geschichte. Sie entscheidet darüber, wie man einer Geschichte folgt - linear, nicht-linear oder interaktiv. Vorläufer solcher navigationsbasierten Erzählstrategien ist der Audio-Walk, welcher vor allem durch die kanadische Künstlerin Janet Cardiff bekannt geworden ist. Ein Audio-Walk ist eine kreative, ästhetische Methode, um einen Ort oder Menschen, dessen Vorstellungswelten und Praktiken näher zu bringen. Durch den Walk wird mit konkreter Anleitung navigiert, was an herkömmliche Leitsysteme erinnert. Im Unterschied zu herkömmlichen Leitsystemen für Städte und Museen, die Sehenswürdigkeiten/Exponate auf einer allgemeinen Ebene zusammenfassen, beinhaltet der Audio-Walk die Möglichkeit, einen subjektiv gefärbten Zugang zu einem Ort zu erhalten. Dabei kommt der Erforschung des Orts eine grosse Bedeutung zu. Janet Cardiff, die oftmals mit ihrem Partner George Bures Miller zusammenarbeitet, beschäftigt sich seit den 1990er-Jahren mit vorgegebenen Räumen. Dabei integriert sie die Umgebung als Kulisse und Soundquelle in ihre Erzählstrategie. Auf ihren Walks vermischen sich Realität mit Fiktion, vorgefundene Geschichten werden mit eigenen Erinnerungen und Gedanken verwoben. Durch eine ausgeklügelte Technik wird das zuvor aufgezeichnete Abschreiten des Raums mit dem Durchschreiten des physischen Raums überlagert, was zu irritierenden Situationen führt. Erreicht wird diese 3-D-Wirkung durch eine binaurale Tonaufnahme mit zwei Mikrofonen in der Ohrmuschel eines Kunstkopfs. Dadurch entsteht ein starker 3-D-Effekt bei der Tonwiedergabe. Oftmals lässt sich bei den Walks auch nicht mehr zwischen abgespielter Geräuschkulisse und den beim Abschreiten des Orts selbst produzierten Geräuschen unterscheiden. Der “Ittinger-Walk» (2002) führt beispielsweise mittels verschiedener Tonspuren und Effekte durch die Kartause Ittingen in der Schweiz. Dabei werden vorgefundene und erfundene Geschichten aus dem Klosteralltag erzählt. 




\section{Design im Wandel}

Die Grundlagen für ein neues Designverständnis wurden eingangs in sieben Punkte - georeferenzierte Daten, Satellitenbilder, GIS-Systeme, visuelle Epistemologien und Netzwerke, Mashups sowie geospatiale Technologien - zusammengefasst und im Theorieteil dieser Publikation ausführlich erörtert und beschrieben. Bevor nun im folgenden Praxisbeispiel anhand einer konkreten Anwendung einzelne Aspekte der besprochenen Themen weitergedacht und iterativ erprobt werden, sollen zunächst die Bezüge nochmals kontextualisiert und in einen grösseren Designbezug gestellt werden. Als ein Jahr vor der Mondlandung den Astronauten der Apollo-8-Mission mit der Serie «Earthrise» ikonische Aufnahmen der Erde gelangen, kommt es schlagartig zu einem allgemeineren Bewusstsein für die Welt als Ganzes und ihrer Verletzlichkeit. Da es der Politik bisher nicht gelungen war, Umweltthemen anzugehen und nachhaltige Lösungen umzusetzen, sollte nunmehr der technologische Fortschritt den voranschreitenden Klimawandel in den Griff bekommen. Die Aufnahme der verletzlichen Kugel im Weltall prägte schon bald das Titelbild des Whole Earth Catalogue, der sinnbildlich für den Fortschritt stand (und oftmals als Vorläufer der Google-Suchmaschine gesehen wird). Das Konzept der Singularität führt zu einer Aufbruchsstimmung und einem allgemeinen Technologie-Hype bei den Start-ups im Silicon Valley und zu den Monopolbildungen der grossen Internetunternehmen (Big Five oder GAFA: Google, Apple, Facebook, Amazon und Microsoft). Das Konzept der Singularität vermochte dem Klima bisher auch nicht weiterzuhelfen, wie das Kyoto-Protokoll und die Klimaproteste um Greta Thunberg (Fridays for Future) verdeutlichen. Im Gegenteil, die energiefressenden Serverfarmer sind inzwischen selbst zu einer grossen Klimabelastung geworden. Aber es hat den Internetkonzernen zu fast uneingeschränkter Macht verholfen. Diese Umverteilung und Konzentrierung von Macht veränder(te)n Privatsphäre, Urheberrechte und geistiges Eigentum fundamental. Einerseits sind proprietäre, hegemoniale Wissensmonopole wie Google Maps entstanden. Andererseits formen sich als Reaktion darauf Creative Commons wie OpenStreetMap. Egal ob proprietäre oder offene Systeme hinter den verschiedenen Technologien und Diensten stehen, gewiss ist, dass sie eine radikale Änderung von Design befördern und damit einen neuen Blick auf das Thema sowie eine überzeugende Designpraxis mit entsprechenden Designmethoden verlangen.

Soll Design, wie der deutsche Philosoph Wolfgang Welsch anlässlich eines Vortrags beim World Design Congress in Nagoya, Japan, einst propagierte, zur Leitdisziplin des 21. Jahrhunderts wer- 
den (Welsch 1990: 218), muss es zwingend mit der Digitalisierung mithalten und den technologischen Wandel aktiv mitgestalten. Damit meine ich nicht nur den Diskurs und die kritische Debatte entlang neuer Technologien, sondern auch die interdisziplinäre Praxis und die Zusammenarbeit mit anderen Disziplinen etwa in der Entwicklung neuer Anwendungen, Funktionen und Oberflächen von Kommunikationstechnologien. Einerseits hat sich Design in den letzten Jahrzehnten zu einer strategischen Disziplin entwickelt (Design Thinking, Kreativwirtschaft, Kreativität, vgl. Florida 2002, 2005; Reckwitz 2014 u. a.) und gilt heute als USP (Unique Selling Point) zur Erschliessung neuer Märkte und Marktabsätze sowie zur Selbstinszenierung (vgl. Böhme 2016 u.a.). Andererseits hat sich Design zu einer Wissensdisziplin gewandelt (vgl. Mareis 2011 u. a.) und mischt sich in gesellschaftliche, politische und ethische Debatten ein. Seit der BolognaReform hat sich Designforschung innerhalb kurzer Zeit in den Curricula der Kunst- und Fachhochschulen etabliert, findet weltweit Beachtung und verfügt über eine aktive Community. Auf viele drängende gesellschaftliche Fragestellungen und Probleme wie zum Beispiel Globalisierung und Klimawandel, Migration und Geopolitik, Big Data und Künstliche Intelligenz ist vermehrt auch eine designzentrierte Perspektive gefragt. Deshalb ist es unerlässlich, neben einer kritischen Haltung auch eine aktive Teilhabe am technologischen Diskurs einzufordern (Creative Coding, Software Engineering, Computational Design u.a.).

Mit einer wie oben thematisierten paradigmatischen Designpraxis werden die Grenzen im Design durchlässiger. Es sind zunehmend inter-, trans- und kulturelle Perspektivierungen gefragt, die die Designdisziplinen integrativ verhandeln und zu nutzen wissen. Entsprechend hoch ist der gesellschaftliche Impact, den ein solch situiertes Design erreichen kann. Nicht nur auf die dringenden Fragen nach dem Wie (Kreativität, Empathie, Kollaboration, Entwurf, Open Access u.a.), sondern auch auf die wesentlichen Fragen nach dem Warum (Fake News, Wissensfilter, Künstliche Intelligenz u.a.) müssen zwingend Antworten gefunden werden. Wenn Design in einer agilen, brüchigen und sich schnell verändernden Welt zunehmend eine prioritäre Rolle beansprucht (wir alle sind Designer_innen, Design durchdringt sämtliche Lebensbereiche, die Welt ist Gegenstand und Ergebnis von Design u. a., vgl.z. B. Reckwitz 2014, 2017), ist es umso dringlicher, empirische Konzepte, Systeme und Kenntnisse zu erforschen.

Was bedeutet das für die Designdisziplinen? Wie verändern sich dadurch Rolle und Verantwortung der Designer_innen? Die Akzeptanz und der sich sehr schnell - seit der Industrialisierung bis hin zum aktuellen gesellschaftlichen Hype Design for Everyone im Sinne 
von «wir alle sind Designer - vollzogene Aufstieg von Design als Gestaltungs- und Wissensdisziplin verlangen nach Verantwortung, danach, eine kritische Position und Haltung einzunehmen. Design in seiner transformierenden Kraft (vgl. Transformationsdesign, Sommer \&Weltzer 2014) sollte innovative Anwendungen und Konzepte entwickeln, um ein neues Technologieverständnis zu fördern und mit dem Wandel einhergehende Bedenken und Ängste vor Kontrolle, Abhängigkeit und Manipulation zu thematisieren. Bereits am Anfang der Designforschung in den 1960er-Jahren wurde deutlich, dass sich Designer_innen nicht mehr allein auf die Fähigkeit verlassen können, die Gestaltung eines Produkts in den Mittelpunkt einer Designaufgabe zu stellen. Aufgrund der technologischen Entwicklungen und mit der Auswirkung der Massenproduktion verlagerte sich das Interesse von Produkt- und Formgestaltung auf die Integration von menschlichen Bedürfnissen. Human-centered Design (Krippendorff 2012; siehe auch Sennett 2008) bildete ein bis heute tragendes Designkonzept. In einer postindustriellen Zeit und mit Industrie 4.0 wird vermehrt für beziehungsweise von Softwareagenten, Computern und Maschinen gestaltet. Das heisst, die Gestaltung muss beispielsweise maschinenlesbar und -verarbeitbar sein. Damit rücken paradigmatisch neue Designkonzepte in den Blickwinkel, welche die maschinenzentrierte Gestaltung (Bratton 2015) oder aber auch die Gestaltung für ganz andere Lebenswelten wie Tiere und Pflanzen (Colomina \& Wigley 2016; Haraway 2018) im Fokus haben werden. Nach dem schwindenden Vertrauen in die Wirtschaft (ausgelöst etwa durch Finanzkrise und Diesel-Skandal) sowie mit dem Erfolg von disruptiven Geschäftsmodellen und Technologien kommt es zu einer Neupositionierung von Design. Design entwickelt sich zunehmend zu einem kritischen Design (vgl. Banz 2016; Borries 2017 u. a.) oder auch zu einer spekulativen (what if) oder politischen Disziplin (Burckhardt 2004; Papanek 2009; Burckhardt 2012; Pater 2016). Durch kritisches Hinterfragen und mit spekulativen Experimenten (vgl. Dunne \& Raby 2014) werden entlang einer designzentrierten Perspektive gesellschaftliche Rahmenbedingung erprobt, verworfen und hinterfragt.

In digitalen Kulturen gilt eine Kritik unter anderem den Daten und ihrer Herkunft und der Distribution und der Speicherung von Informationen. Mit der Annahme der umstrittenen Vorlage und stark umkämpften Abstimmung zur EU-Datenschutz-Grundverordnung gelten neue Gesetze, die die Urheber_innen von Daten besser schützen werden. Denn neben den utopischen Verheissungen und der Technologiegläubigkeit gibt es vermehrt auch Gegenstimmen, die insbesondere in Design und Kunst laut werden. So beschwört zum Beispiel der britische Künstler James Bridle in seinem Buch New 
Dark Age (2018) ein neues Mittelalter herauf, in welchem wir trotz der grossen Verfügbarkeit von Daten und Informationen zunehmend in Unwissen respektive Abhängigkeit leben (für andere kritische Stimmen siehe auch Foer 2018; Greenfield 2018; Zuboff 2018 u. a.).

Grosse Teile der Technologie-Kritik betreffen dann auch die Komplexität von Daten und Informationen. Design kann dieser Herausforderung darin begegnen, indem das Gestalten und das Aufbereiten von Daten und Informationen zur Aufgabe werden. Der Designer und Künstler Lev Manovich plädiert dann auch dafür, nicht (noch mehr originäre) Daten zu generieren, sondern bestehende Daten zu indexieren und in einen Kontext zu stellen.

Einen weiteren grossen Handlungsbedarf gibt es hinsichtlich Ästhetik, Interaktion und dem Erlebnis von Oberflächen und hybriden Räumen. Durch den Einsatz von interaktiven Kommunikations- und Medientechnologien im öffentlichen Raum gehört der (urbane) Raum heute zum Aufgabenbereich der Designer_innen, insbesondere im Bereich von Navigation und Kommunikation. Dabei geht es neben der medialen Erscheinung des Interface - bedingt durch die komplexe Informationsvermittlung - vermehrt auch um die Gestaltung von Räumen und Erlebnisse (vgl. Russegger/Tarasiewicz/Wlodkowski 2011; Denzinger 2018 u.a.). Vor diesem Hintergrund sollte Design mit der Gestaltung räumlicher Situationen auf die gegenwärtige Verräumlichung von Informationen und Daten Einfluss nehmen beziehungsweise solche aktiv mitgestalten.

Mit ortsbasierten Diensten respektive geospatialen Technologien zeichnet sich eine Hinwendung zum Raum, zu räumlichen Themen sowie zu räumlichen Gestaltungsdisziplinen ab, was sich einerseits in der disziplinären Ausrichtung zeigt (Interface Design, Interaction Design, Usability Design, Informationsdesign, Datenvisualisierung u.a.) sowie andererseits in der Designpraxis (von der Gestaltung von Graphical-User-Interfaces über bildschirm- und raumbasierte Medien bis hin zu gestischer Interaktion u.a.). Die Dringlichkeit von Karten und Kartentechnologie als neue Interfaces sowie der Einfluss von gestalterischen Strategien und Konzepten versprechen ein grosses Potenzial für die Designdisziplinen. Die Herausforderungen im Design etwa bezüglich Urheberschaft, Konformismus, Datenschutz, In- und Ausschlüssen, kognitiven und physischen Implikationen sind ebenso gross. Mit der «Verräumlichung von Wissen ist Design zunehmend gefordert, visuelle Standards zu (er)finden und in Zusammenarbeit mit Ingenieur_innen, Creativ Coder_innen und Programmier_innen neue Visualisierungskonzepte und Designprozesse in Anbindung an Netzwerke, Sensoren und Dienste zu entwickeln. 


Anwendung 

Augmented Space

Am Beispiel des Zollfreilagers auf dem Dreispitz 


\section{Das Basler Zollfreilager und die Praxis der Kunstfreilager}

Freilager sind Orte zur zoll- und abgabefreien Lagerung von Wertgegenständen, bevor diese importiert oder exportiert werden. Auf die dort gelagerten Waren werden bis zu ihrer Einführung keine Zölle oder Steuern erhoben. Solche Freibezirke sind steuertechnisch ein Niemandsland und gelten als exterritorial beziehungsweise als Zollausland. Das Basler Zollfreilager umfasste ein Zollfrei- und ein Transitlager, eine Zollverwaltung, drei Zollstationen, mehrere Lagerhallen, einen Inlandparkplatz sowie Rangieranlagen mit einem engmaschigen Schienen- und Strassennetz zur Ein- und Ausfuhr der Waren. Auf die Nutzung des Areals als Güterumschlagplatz verweisen noch heute die zahlreichen nach (Handels-) Städten benannten Strassen, ein 
Lastenkran, Bahngeleise sowie das zum Ateliergebäude der Hochschule für Gestaltung und Kunst FHNW umgenutzte Zollfreilager. Ausgehend von einer Raum- und Nutzungsanalyse des Basler Zollfreilagers sowie von Zollfreilagern allgemein wird das historische Areal erforscht sowie zum einen die Praxis der Kunstfreilager untersucht, zum anderen näher beleuchtet, wie modernste Infrastrukturen und exterritoriale Steuerzonen die wirtschaftliche und gesellschaftspolitische Realität prägen.

In fast jedem Land gibt es Zollfreilager, doch nur eine geringe Anzahl ist massgeblich in den internationalen Kunsthandel involviert. Die wenigen - allen voran der Genfer Freihafen haben jedoch für weitreichende Schlagzeilen gesorgt. Zollfreilager, hier im spezifischen Kunstfreilager, sind zu dynamischen 
Knotenpunkten für globale Güterund Finanzkreisläufe geworden und werden zur Steuervermeidung verwendet. Die Sonderhandhabung und Duldung durch den Staat dienen der Autorisierung und Sicherung eines globalen Kunst-Kapitalismus. 


\section{Das Basler Zollfreilager}

Auf dem Gelände des heutigen Campus der Künste befand sich von 1922 bis 2008 das Basler Zollfreilager ${ }^{40}$. Das Zollfreilager war Teil des sogenannten Dreispitz-Areals, einem Gebiet südwestlich der Basler Innenstadt, auf welchem sich seit 1901 ein öffentliches Materiallager befand. Das Land gehörte Christoph Merian (1800-1858) und ging nach dem Tod seiner Frau Margaretha (1806-1886) an die Christoph Merian Stiftung (CMS). Seither verwaltet die Stiftung den Landbesitz und verpachtete das Land an die ansässigen Betriebe. Um 1901 gab es zwölf eingemietete Firmen, 1914 waren bereits 30 Unternehmen angesiedelt und bis etwa 1951 stieg die Zahl auf über 200 Firmen. Die Unternehmen waren aufgeteilt in Handelsbetriebe (53 Prozent), Speditions- und Lagerhausbetriebe (25 Prozent), Industrie-, Gewerbe- und Handwerksbetriebe (20 Prozent) sowie öffentliche Betriebe (2 Prozent). In den 1950er-Jahren lag der Wert aller Gebäude und Warenvorräte auf dem Areal bei rund 300 Millionen Schweizer Franken bei einer Nutzfläche von circa 300000 Quadratmetern.

Um den wachsenden Waren- und Güterstrom bewältigen zu können, fasste der Bundesrat um 1920 den Beschluss, auf dem Areal ein Lagerhaus zur Lagerung von zollfreien Gütern zu errichten. 1922 erfolgte die Gründung der Basler Freilager-Gesellschaft, die ab 1923 ihren Betrieb aufnahm. Zur Errichtung des Freilagers stellte die Christoph Merian Stiftung eine Parzelle von rund 50000 Quadratmetern im Baurecht zur Verfügung. Die Architekten Widmer, Erlacher \& Calini wurden mit dem Bau des Zollfreilagers, eines Lagerhauses sowie eines separaten Gebäudes für die Zollverwaltung inklusive einer Zollstation beauftragt. Die Zollverwaltung umfasste neben Büros und einem Wachlokal auch Wohnungen für die Zollbeamten. Im neu erstellten Zollfreilager wurde fast die ganze erste Etage an die Leder-Import AG vermietet. In dieser ersten Bauetappe wurden rund 29000 Quadratmeter des Areals genutzt. Bereits ein Jahr später wurden Erweiterungen vorgenommen: Das Lagerhaus wurde vergrössert sowie ein weiteres Lagerhaus erstellt. Die Lüchinger \& Cie. AG errichtete ein modernes Kühlhaus für Eier und diverse Laderampen für die Warenanlieferung wurden gebaut. Die Speditionsfirma Goth \& Co mietete sich ein und betrieb einen Lagerplatz für Autos. Hierfür wurde ein elektronischer Laufkran von 55 Metern Länge und mit einer Tragfähigkeit von fünf Tonnen installiert. (Abb.58) Als der Laufkran nicht mehr ausreichte, kam ein mobiler

40 Zur Geschichte des Basler Zollfreilagers siehe auch: Basler Freilager AG 1947; Verwaltung der Oeffentlichen Materiallagerplätze Basel-Dreispitz 1951; Wanner 1972. 


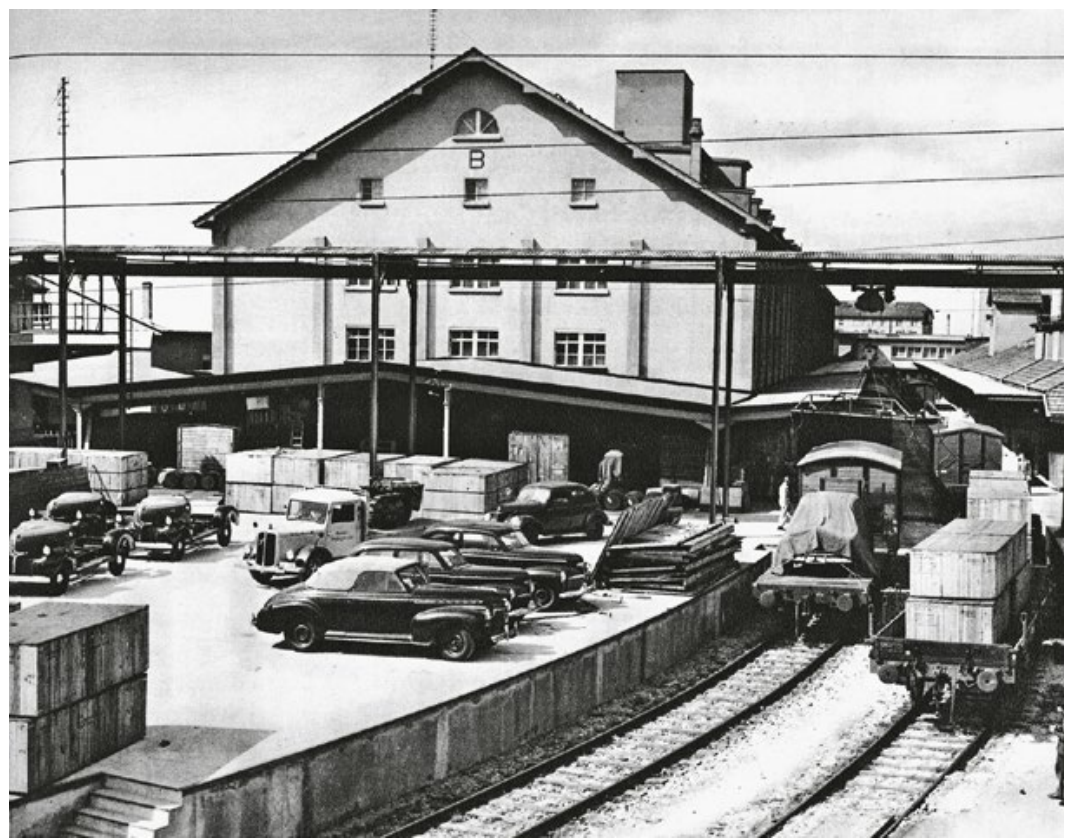

Kran hinzu. Zu diesem Zeitpunkt wurden inzwischen rund 40000 QuadratDas Basler Zollfreilager auf dem Dreispitz-Areal um 1930 meter des Areals genutzt. Die Roba Schiffahrts Agentur-und Lagerhaus-AG sowie die Chambre Arbitrale et de Conciliation pour Laines et Peignés und die Firma Roessiger \& Cie. mieteten sich ein. Der Bedarf an neuen Lagerflächen führte zu weiteren Erweiterungen und zum Bau des Lagerhauses. Damit hatte das Freilager 1947 seine Fläche von rund 50000 Quadratmetern ausgeschöpft, davon rund 25000 Quadratmeter für Gebäude und Lager.

1952 wurde das Areal auf rund 75000 Quadratmeter aufgestockt; davon rund 56000 Quadratmeter im Baurecht beziehungsweise als Baureserve entlang der Emil-Frey-Strasse. 1959 entstand dort der neue Inlandparkplatz mit angrenzendem Zollposten. Zehn Jahre später erfolgte durch die neu gegründete Transitlager-AG (einem $\mathrm{Zu}$ sammenschluss der Basler-Freilager AG, der Schweizerischen Bundesbah- 


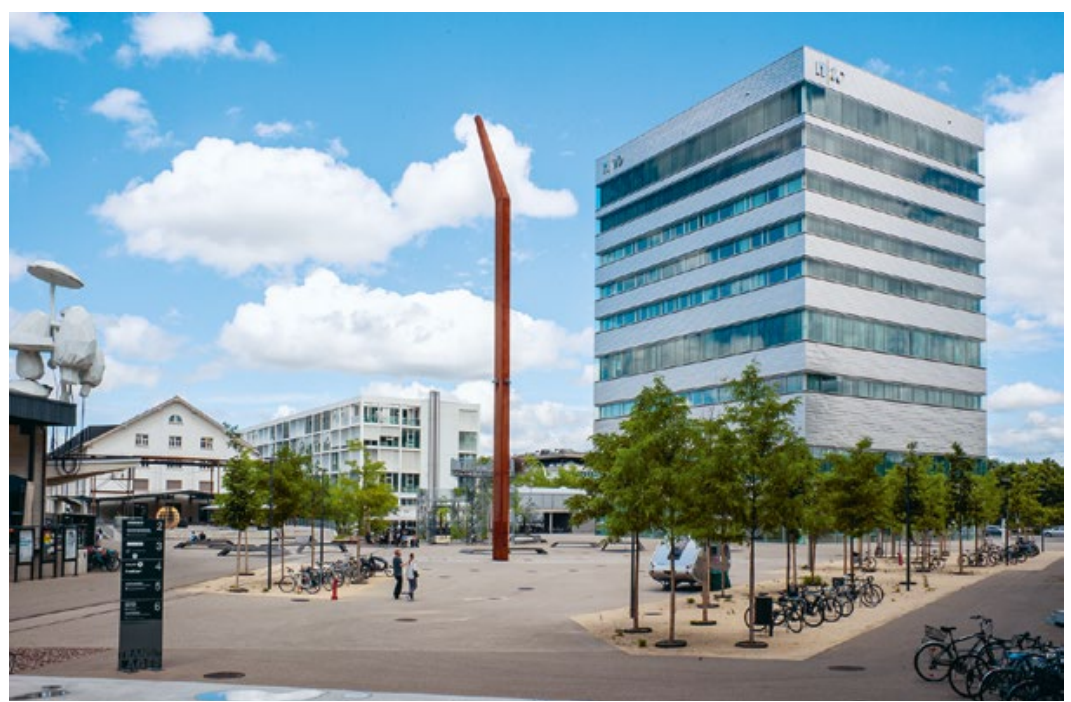

nen sowie der Handelskammer Basel) die Erstellung des Transitlagers. Das Der Campus der Künste auf dem Gelände des ehemaligen Zollfreilagers mit dem neuen Gebäude umfasste fünf Stockwerke Hochhaus (2015) mit einer Lagerfläche von rund 15000 Quadratmetern. Weitere Verträge im Unterbaurecht gingen an die Distroptic AG und Roba AG (1963), die Basler chemische Industrie (1964) sowie an die Natural AG (1970). Mit der Liberalisierung der Verzollungsformalitäten von 1985 verlor das Basler Zollfreilager wie alle Freilager - in seiner damaligen Form an Bedeutung (für den In- und Export von Gütern). Um das Jahr 2000 wurde es aufgegeben und seit 2015 befinden sich auf dem Areal unter anderem die Hochschule für Gestaltung und Kunst FHNW (Abb.59), das Haus der Elektronischen Künste sowie die Bibliothek der Schule für Gestaltung Basel. Das Land gehört weiterhin der Christoph Merian Stiftung und wird im Baurecht auf jeweils 100 Jahre an die Mieter_innen verpachtet. 


\section{Zollfreilager und Freihandelszonen}

Der Schweiz kommt in Sachen Zollfreilager eine Pionierrolle zu. In dem kleinen Land gibt es sieben aktive Zollfreilager und 245 offene Zolllager. ${ }^{41}$ Offene Zolllager unterscheiden sich von Zollfreilagern dadurch, dass bei diesen der Zoll nicht vor Ort ist und daher nur sporadisch Stichproben stattfinden. Deshalb leisten offene Zolllager üblicherweise eine Sicherheit in Höhe von 2 Prozent auf den gelagerten Warenwert. Die darin gelagerten Güter wurden 2013 von der Eidgenössischen Finanzkontrolle auf einen Gesamtwert von etwa 15 Milliarden Schweizer Franken geschätzt.

Freilager und Freihäfen lassen sich bis ins Mittelalter zurückverfolgen. Sie entstanden in Hafengegenden, mit Aufkommen der Schienen- und Luftfracht später in der Nähe von Bahnhöfen oder Flughäfen, wo sie noch heute zu finden sind. Der eigentliche Zweck von Zollfreilagern ist die Lagerung von Gütern vor deren Einfuhr in ein Land. Denn erst bei Einführung der Ware fallen Zölle und Steuern an. Zollfreilager sind entsprechend Dienstleistungsbauten, die der Überwachung durch die Zollverwaltung unterstehen. Freihandelszonen werden von verschiedenen Organisationen wie zum Beispiel der Financial Action Task Force (FATF) überwacht, der auch die Schweiz angehört. Viele der Zollfreilager sind mittlerweile zweckentfremdet, da seit der Revision des Zollgesetzes Güter dauerhaft zollfrei gelagert werden dürfen. Ursprünglich gedacht, um Warenströme zu erleichtern, werden Freilager heute allerdings zur Dauerlagerung von Wertgegenständen und Luxusgütern genutzt. Die eingelagerte Ware kann durch Banken belehnt werden, das Zollfreilager übernimmt gegebenenfalls die Treuhänderschaft. Freilager sind zu einem weltweiten Aufbewahrungsort und einem globalen Investitionssystem für den Kauf und Verkauf von Kunst geworden. Mit der Abschaffung des Schweizer Bankengeheimnis, dem weltweiten Zusammenbruch der Finanzmärkte (Negativzinsen und niedrige Rendite bei Finanzanlagen) und dem allgemeinen Misstrauen in das Bankensystem boomen der Kunstmarkt und die Kunstfreilager. Solange die Kunstwerke unauffällig im Depot bleiben, lassen sich Vermögen-, Zins- oder Erbschaftsteuerzahlungen vermeiden.

Da der Kunstmarkt kaum mehr reguliert wird, ist auch viel Schwarzgeld und Raubkunst (zur Geldwäscherei und Terrorismus-Finanzierung) im Umlauf. Inventare und Details zu Besitzverhältnissen sind zwar in der Schweiz vorgeschrieben, jedoch schwer überprüfbar, da Logistik-Firmen dazwischenstehen. Dadurch sind Zollfreilager

41 www.ezv.admin.ch/ezv/de/home/information-firmen/waren-anmelden/ einfuhr-in-die-schweiz/zollfreilager.html (abgerufen am 11.05.2018). 
anfällig für illegale Geschäfte wie Steuerhinterziehung, Geldwäscherei oder das Lagern von Raubkunst. In der Schweiz stellt die Eidgenössische Finanzkontrolle unter anderem immer wieder Fehler bei der Warenanmeldung, Unregelmässigkeiten bei der Bestandsaufnahme sowie Lücken in der Lagerbuchhaltung fest, die der Steuerhinterziehung und der Geldwäscherei dienen. Gruppierungen wie die Organisation für wirtschaftliche Zusammenarbeit und Entwicklung (OECD) oder die Groupe d'action financière (GAFI) weisen auf diese undurchsichtigen Praxen im Zusammenhang mit den Zollfreilagern hin. Die Anonymität der privaten Lagerhausgesellschaften (oftmals sind Treuhandfirmen als Dienstleister zwischengestellt) erschwert die Fahndung und das Aufdecken von Delikten. Diese Anonymität bezieht sich nicht nur auf die Lagerung von Kunstwerken, sondern auch auf den Kauf und Verkauf. Dadurch, dass die Käufer_innen und Verkäufer_innen (in sogenannten Private Sales bzw. Private Collections) anonym bleiben, wird Diskretion garantiert.

\section{Der Kunstmarkt}

Das Werk “Porträt eines Künstlers» von David Hockney erzielte im Jahre 2018 bei einer Versteigerung bei Christie's einen Preis von 90 Millionen US-Dollar. “Rabbit» von Jeff Koons wurde kürzlich vom gleichen Auktionshaus für 90,3 Millionen US-Dollar versteigert - der bisher höchste jemals bezahlte Preis für das Werk eines lebenden Künstlers. Zeitgenössische Kunst erreicht damit nicht nur absolute Spitzenpreise, auch wird Kunst inzwischen überwiegend kommerziell vermarktet. So sorgte etwa die Zusammenarbeit von Koons mit dem Luxuslabel Louis Vuitton für dessen Taschenkollektion für Aufsehen. (Abb. 60) Vielleicht die Spitze der Kommerzialisierung und Veralberung kann in der goldenen Toilette "America" des italienischen Künstlers Maurizio Cattelan gesehen werden, die Scharen von Besucher_innen ins Guggenheim Museum nach New York lockt.

Mit der Kommerzialisierung und durch schnelles Kaufen und Verkaufen (Art Flipping) wurde Kunst, vor allem zeitgenössische Kunst, zu einem Spekulationsobjekt (niemand kann sagen, wie sich bei zeitgenössischer Kunst der Markt entwickeln wird und ob ein Werk in einigen Jahren das Zehnfache oder weniger als der Einstandspreis wert sein wird). Angeheizt wird dieser Trend dadurch, dass immer mehr reiche Leute und Stars in Kunst investieren (oftmals ohne wirkliches Interesse oder einer Passion für die Kunst zu haben). Das Kunstsammeln wird zum Lifestyle und zur Unterhaltung zele- 
briert. Neben professionellen Sammler_innen wie Yusaku Maezawa oder Larry Gagosian machen es Stars und Sternchen wie Jonny Depp, Leonardo DiCaprio oder Madonna vor und inszenieren ihre Kunstaffinität in den sozialen Medien. Basierend auf Interviews mit Sammler_innen, Kurator_innen, Händler_innen, Auktionshäusern und Museen (und nicht anhand der höchsterzielten Preise), erhebt das Online-Magazin ARTnews ${ }^{42}$ jedes Jahr eine Umfrage zu den weltweit Top-200-Sammler_innen. Aus der Umfrage geht hervor, dass zehn der grössten Kunstsammler_innen einen Wohnsitz in der Schweiz haben.

Auch wenn der Kunstmarkt für zeitgenössische Kunst als überhitzt gilt und die Blase mehrfach geplatzt ist zum Beispiel die der aufstrebenden Künstler_innen in den Jahren 2014 und 2016 (vgl. Adam 2017: 160) -, gibt es nach wie vor einen grossen Hype um das Kunstsammeln als Anlageform. Und natürlich gibt es wie immer, wenn es um viel Geld geht, umtriebige Geschäftsleute, die in der Überhitzung des Kunstmarkts das grosse Geschäft wittern. Mit der umstrittenen Website ArtRank hat der ehemalige Galerist und Informatiker Carlos Rivera einen Service geschaffen, mit welchem er seinen Kunden_innen Empfehlungen zum Kauf und Verkauf der Werke von Künstler_innen gibt, als ob es sich bei deren Kunstwerken um Aktien handeln würde..$^{43}$ Seine Anlageempfehlungen gehen auf einen Algorithmus zurück

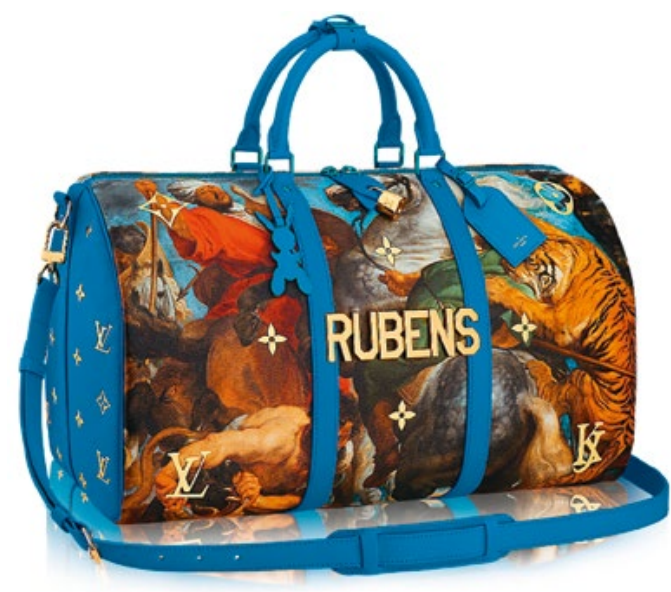

Die in Zusammenarbeit von Jeff Koons mit Louis Vuitton entstandene Tasche

42 www.artnews.com.

43 Siehe hierzu auch www.theguardian. com/artanddesign/2014/jun/23/artrankbuy-sell-liquidate-art-market-websiteartists-commodities 
und basieren auf Daten aus sozialen Netzwerken wie Twitter oder Instagram und aus den Auktionsresultaten und Ausstellungsaktivitäten der Künstler_innen. (Abb.61) Mittels dieses Algorithmus quantifiziert Rivera die Kunstwerke und versucht, kommende Trends und Blasen auszumachen. Viele der gelisteten Künstler_innen sind entsetzt über sein Vorgehen und die Bewertung ihrer Person und Kunstwerke auf einer Website. Rivera selbst findet nichts dabei, Kunst zu bewerten, schliesslich sei das der normale Alltag eines Kunstberaters beziehungsweise eines Galleristen.

Die Popularität zeitgenössischer Kunst zeigt sich auch anhand der weltweiten internationalen Kunstmessen und Grossausstellungen wie etwa Frieze, Art Basel (mit Miami Beach und Hongkong) oder ACROmadrid. Mit der Art Basel beheimatet die Schweiz eine der bedeutendsten Kunstmessen weltweit. Laut UBS-Report wurde im Jahre 2016 der Umsatz des Kunstmarkts auf 56,6 Milliarden US-Dollar geschätzt. Insgesamt wurden für rund 13,3 Milliarden US-Dollar auf Kunstmessen Kunstwerke verkauft, was einer stetigen Zunahme zu den Vorjahren entspricht. Die USA haben mit 40 Prozent Anteil den höchsten Umsatz, gefolgt von Grossbritannien mit 21 Prozent und China mit 20 Prozent. Mit dem Erwerb der Kunst braucht es auch Lagerorte, in welchen die Werke sicher aufbewahrt werden können. Denn die wenigsten der erworbenen Kunstwerke werden privat aufbewahrt oder öffentlich ausgestellt. Gemäss Schätzung sind weltweit rund 80 Prozent der Kunstwerke zu einem bestimmten Zeitpunkt

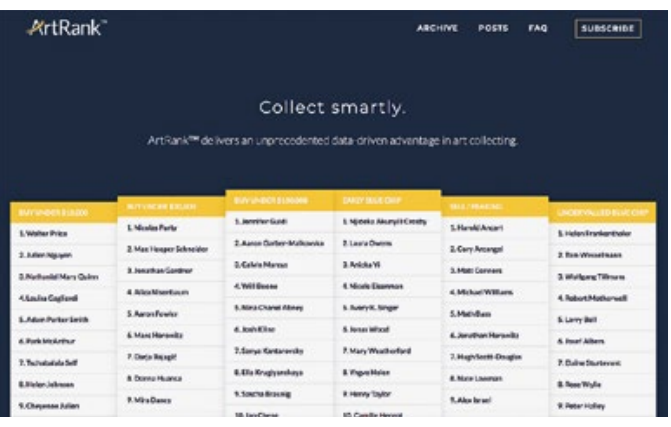

Die Website ArtRank von Carlos Rivera mit Empfehlungen zum Kauf von Künstler_innen 
eingelagert (vgl. Simon Hornby, zit.n. Adam 2017: 132). Zur Aufbewahrung sind bei Sammler_innen sowie Galerien, Museen und Anlagefonds besonders private Lagerhausgesellschaften in Zollfreilagern beliebt. Laut dem britischen Magazin The Economist wird der Wert der weltweit gelagerten Kunstwerke auf mehrere hundert Milliarden US-Dollar geschätzt. Allein schätzungsweise einige Milliarden Schweizer Franken sollen in Form von Kunstwerken in den Zollfreilagern lagern - steuerfrei und mehrheitlich unkontrolliert. ${ }^{44}$ Laut New York Times werden im Genfer Freihafen tausend Picassos vermutet, die alleine ein ganzes Museum füllen würden. Die Künstlerin Hito Steyerl spricht gar von "Tausenden Picassos », obwohl aufgrund der undurchsichtigen Dokumentation niemand eine genaue Anzahl nennen kann und das Werkverzeichnis von Picasso nicht mehr als 1885 Werke fasst (vgl. Steyerl 2015: 3). Kunstfreilager sind - wie das einstige Schweizer Bankgeheimnis - gut gehütete Geheimnisse; diskret, sicher und verhältnismässig günstig. Die Kunstwerke bleiben über Jahre oder Jahrzehnte in den Freilagern und werden dadurch der Öffentlichkeit und dem Markt entzogen. Durch die Lagerung liegen Güter von hohem Wert brach und warten auf ihren Wiedereintritt in das System der globalen Wirtschaftsnetzwerke. Die Kunstwerke sind weder Teil einer öffentlichen Privatsammlung, eines Museums oder einer Galerie, sondern Lagerbestände in Depositorien. Viele Kunstwerke von hohem kulturellen Wert verschwinden so hinter Mauern, wie beispielsweise Cy Twombly "Leda and the Swan" (1962), das für 52,9 Millionen US-Dollar von Larry Gagosian bei Christie's ersteigert und seit 25 Jahren nicht mehr öffentlich gezeigt wurde.

Das 21. Jahrhundert kann als eine Zeit der Lagerung von Kunst bezeichnet werden - wohl noch nie wurden weltweit so viele Kunstwerke gelagert. Zollfreilager, die sich insbesondere auf die Aufbewahrung von Kunst spezialisiert haben, werden derzeit immer beliebter, was die weltweiten Projekte und Planungen neuer Standorte verdeutlichen. Einst als temporäre Lagerorte für den Handel gedacht, sind heutige Freilager diskrete Sicherheitsbunker, die die Kunstwerke vor Diebstahl, Beschädigung oder Verlust schützen. Um Käufe abzuwickeln, verfügen Freilager über eigene Showrooms sowie geschultes Personal. Bei einem Kauf vor Ort entfallen die Transaktionskosten. Zölle und Steuern (Einfuhr- bzw. Ausfuhrsteuer) fallen erst beim Import an. Der Verkauf innerhalb von Zollfreilagern ist auch von der Mehrwertsteuer befreit. Da die in den Zollfreilagern gelagerten Kunstwerke meist nicht versichert sind, gibt es keine verbind-

44 www.economist.com/briefing/2013/11/23/uber-warehouses-for-the-ultra-rich (abgerufen am 03.02.2019). 


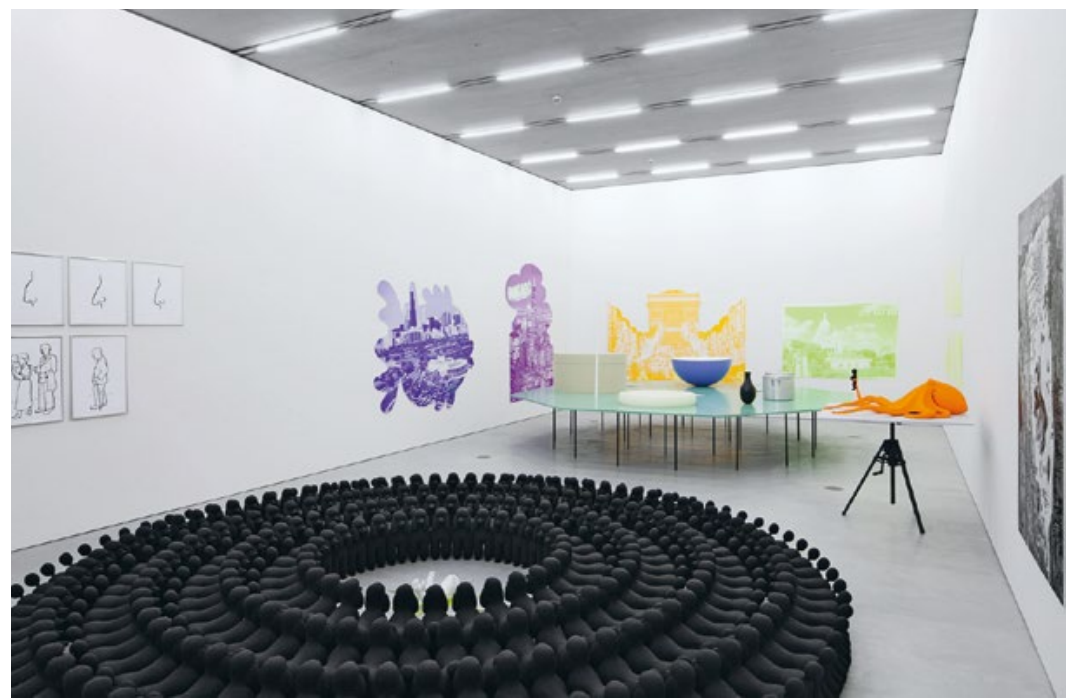

lichen Daten über Wert und Umfang der Sammlungen. Die Zirkulation von Blick in einen Lagerraum des Schaulagers in Basel, in dem sich Teile aus der Sammlung der Emanuel Kunstwerken ist auch aus kostentechHoffmann-Stiftung befinden nischen Gründen rationalisiert. Viele der Werke sind nur noch schwer gegen Verlust oder Beschädigungen zu versichern. Das Risiko ist einfach zu gross, die Versicherungskosten für Transport und Ausstellungen nicht mehr bezahlbar. Aus diesen Gründen kann es für private Sammler_innen interessant sein, ihre Sammlung oder Teile davon in öffentlichen Institutionen zu präsentieren. Das Sammlerpaar Stefan Edlis und seine Frau Gael Neeson vermachte einen Teil ihrer hochkarätigen Kunstsammlung dem Art Institute of Chicago und somit der Öffentlichkeit. Die Sammlung umfasst Werke von unter anderem Cy Twombly, Andy Warhol, Maurizio Cattelan und Katharina Fritsch.

Zwei öffentlich zugängliche Kunstarchive befinden sich auch unmittelbar auf dem Dreispitz-Areal, auf welchem sich das Basler Zollfreilager befand: das durch die Schweizer Architekten Herzog und De Meuron gebaute 
Schaulager (Abb.62) sowie das Depot des Museums der Kulturen. Die Sammlungen sind eingeschränkt und nur nach Anmeldung zugänglich.

\section{Nationale und internationale Zollfreilager mit Kunstbezug}

Das umstrittenste Zollfreilager, das regelmässig in Kunstskandale verwickelt ist, ist zugleich auch das älteste Zollfreilager in der Schweiz. Der um 1888 entstandene Genfer Freihafen, der von Ports Francs et Entrepôts de Genève S. A. betrieben wird, ist eines der Gebäude mit dem wertvollsten Inhalt. Laut der Kulturzeitschrift Connaissances des Arts waren dort im Jahre 2014 rund 1,2 Millionen Kunstwerke gelagert, deren Gesamtwert auf 100 Milliarden US-Dollar geschätzt wird (vgl. Bonnett 2016: 157). Der Freihafen machte mehrmals mit negativen Schlagzeilen auf sich aufmerksam, unter anderem im Zusammenhang mit dort entdecktem Raubgut. 1995 wurde bekannt, dass im Genfer Freihafen ein Netzwerk für geraubte Antiquitäten betrieben wurde, das mit dem Getty Museum in Los Angeles in Verbindung stand. 2003 fanden Schweizer Zöllner 200 antike Schätze aus Ägypten, die als gestohlen gemeldet waren. Nach den Skandalen wurde das Zollgesetz angepasst und seitdem gelten strengere Vorschriften. Im Prinzip muss nun jedes Objekt, das eintrifft oder das Lager verlässt, inventarisiert werden. Doch in der Praxis lässt sich dies leicht umgehen. Die sieben sich in der Schweiz befindenden Zollfreilager stehen in Embrach, St. Margreten, ZürichFlughafen, Chavornay, Genève La Praille, Genève Aéroport und Chiasso. ${ }^{45}$ Diese nationalen und internationalen Zollfreilager sind mit eigenständigen Kleinstaaten vergleichbar, die einer OffshoreBasis gleichen. Drei spektakuläre Fälle - die Bouvier-RybolowlewAffäre, der Fall Schwarzenbach sowie Modigliani und die PanamaPapiere -, die alle mit der Schweiz beziehungsweise dem Genfer Freihafen in Verbindungen stehen, sorgten in den letzten Jahren weltweit für negative Schlagzeilen und Kritik an der Praxis der Kunstfreilager.

Das Konzept der weltweiten Kunstfreilager geht auf den Genfer Freihafen zurück. Als einer der ersten Unternehmer erkannte der Schweizer Kunsthändler Yves Bouvier das enorme Potenzial von auf Kunst spezialisierter Freilager. Mit dem Logistikunternehmen

45 Auf www.ezv.admin.ch/ezv/de/home/information-firmen/waren-anmelden/ einfuhr-in-die-schweiz/zollfreilager.html (abgerufen am 01.02.2018). 


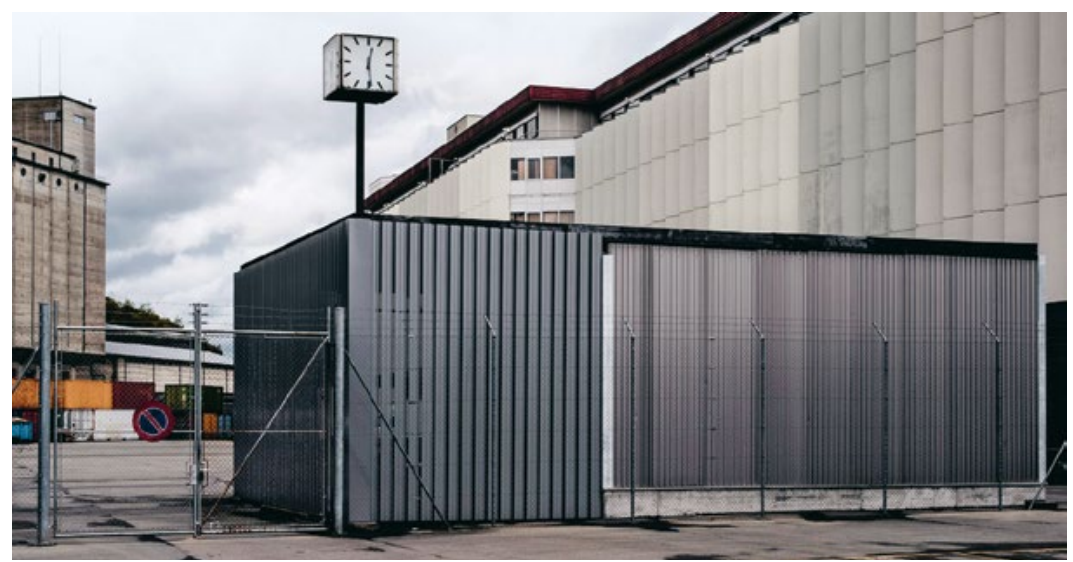

Das Genfer Zollfreilager Ports Francs et Entrepôts de Genève S. A.

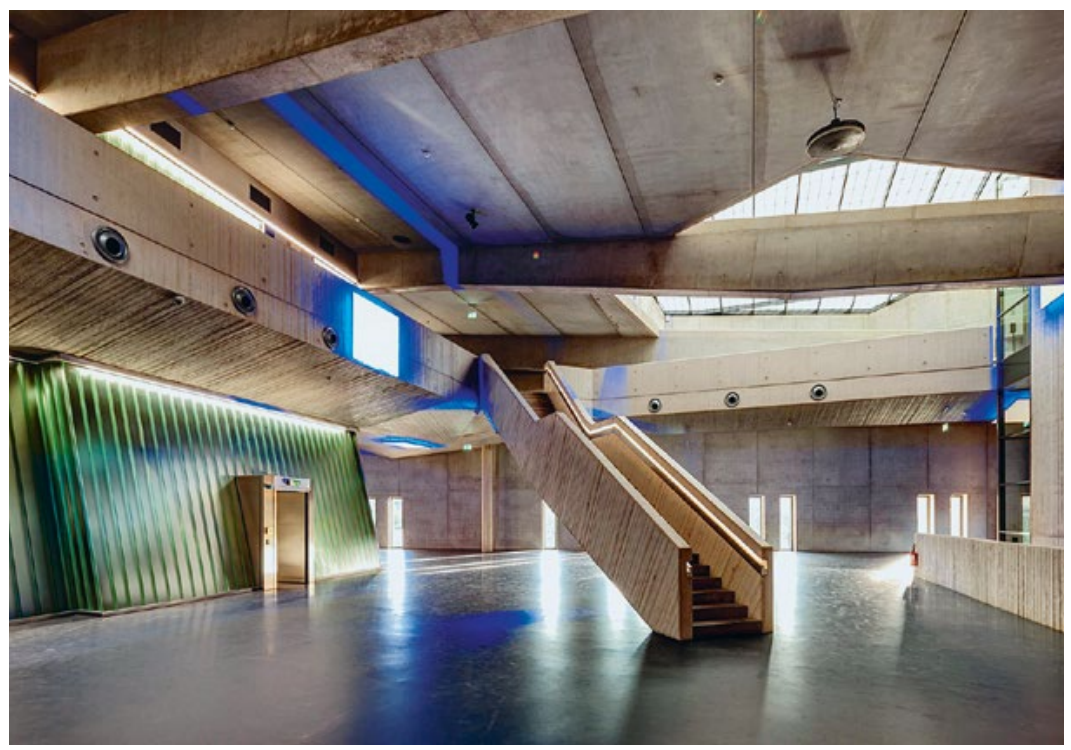

Natural Le Coultre S. A., das auf Lagerung und Transport von Kunstwerken Das Zollfreilager Luxembourg der Gruppe Le Freeport Management Pte Ltd spezialisiert ist, hat er sich ein globales Imperium aufgebaut. (Abb. 63) Nach dem Vorbild des Genfer Freihafens eröffnete Bouvier 2010 das Kunstfreilager Le Freeport (Le Freeport Management Pte Ltd), in Singapur auch Art Fortress in the Heart of Asia genannt, das aufgrund der hohen Sicherheitsstandards, der extravaganten Architektur sowie der auf die Bedürfnisse der Kunstsamm- 
ler_innen ausgerichteten luxuriösen Ausstattung sofort zum Geschäftserfolg und Vorbild weiterer Kunstfreilager wurde. Im September 2014 eröffnet die Freeport-Gruppe nach gleichem Model den Le Freeport in Luxemburg. (Abb. 64) Weitere Freeports sind in Shanghai und Dubai in Planung, um die aufstrebenden Märkte in China und im Nahen Osten zu bedienen. Weitere auf Kunst spezialisierte Zollfreilager sind Beijing freeport of Culture /GeHua Culture Development Group), Monaco Freeport (S.E.G.E.M. Société d'Exploitation et de Gestion des Entrepôts de Monaco) und das ZentralDepot in Meiningen (vallor operations gmbh). In den USA befinden sich Delaware Freeport in Newark und ARCIS Fine Art + Collection Care in New York. Das Freilager in New York, Harlem, hat gar den Status einer FTZ (Foreing Trade Zone), da die USA ein eigenes Steuersystem haben. Kennzeichnend für alle Standorte ist, dass sie sich in der Nähe eines (internationalen) Flughafens befinden oder sogar im Transitbereich. Aufgrund dieser Lage können Kunstwerke nach einem Kauf eingeflogen und zu minimalen Gebühren gelagert werden, ohne dass Steuer- oder Importkosten anfallen. Diese werden erst fällig, wenn das Kunstwerk das Zollgebiet verlässt und in ein Land eingeführt wird. Die interessierte Käuferschaft kann aufgrund der Lage ohne Visa eingeflogen werden.

\section{Die Bouvier-Rybolowlew-Affäre ${ }^{46}$}

Bis 2017 war Yves Bouvier Inhaber des auf die Lagerung und den Transport von Kunstwerken spezialisierten Logistikunternehmens Natural Le Coultre S. A. Aufgrund eines langwierigen und teuren Rechtsstreits mit dem russischen Oligarchen Dimitri Rybolowlew musste Bouvier sein Logistikunternehmen jedoch verkaufen. Vieles in der BouvierRybolowlew-Affäre ist bisher ungeklärt, klar jedoch ist: Im Jahre 2002 oder 2003 lernen sich Bouvier und Rybolowlew im Genfer Zollfreilager kennen. Rybolowlew (Besitzer des Fussballclubs AS Monaco) ist zu diesem Zeitpunkt ein neuer Player im Kunstmarkt und besorgt, weil er für den soeben getätigten Kauf eines Chagalls keine Zertifizierung hat. Bouvier besorgt ihm die Papiere und bringt Rybolowlew dadurch dazu, ihm zu vertrauen. Während der kommenden zehn Jahre baut Bouvier die Kunstsammlung von Rybolowlew auf und kauft in seinem Namen für mehr als zwei Milliarden US-Dollar Werke von unter anderem van Gogh, Picasso, Modigliani, Monet, El Greco, Rothko. In der Zeit von 2003 bis 2007 vermittelt Bouvier Rybolowlew sechs Kunstwerke, zwischen 2008 und 2013 sind es wei-

46 Für die drei folgenden Stories wurde bei dem Online-Nachrichtendienst Reuters sowie dem Online-Portal artnet recherchiert. 


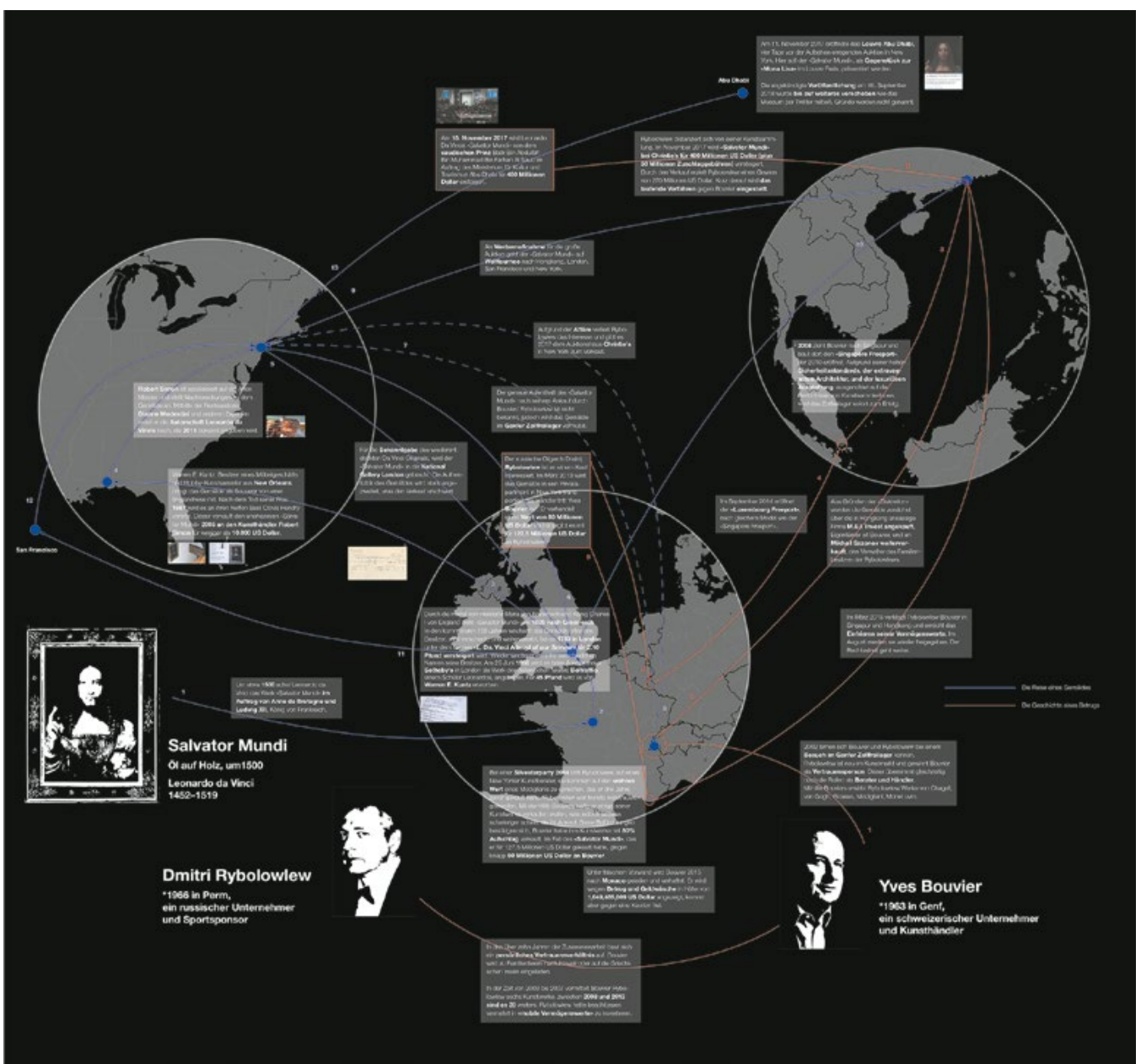

tere 28 (vgl.Adam 2017: 197f.). Aus Gründen der Diskretion werden die Gemälde zunächst über die in Hongkong Die Bouvier-Rybolowlew-Affäre wurde zunächst visuell aufbereitet und danach medienadäquat für die Augmented-Reality-Applikation ansässige Firma M.E.I.Invest angekauft, deren Eigentümer Bouvier ist, und an Mikhail Sazonov weiterverkauft, den Verwalter des Familienbesitzes der Rybolowlews. (Abb. 65) Zwischen den beiden Männern baut sich ein enges Vertrauensverhältnis auf. Bouvier wird zu Familienfeiern nach Hawaii und auf die griechischen Inseln eingeladen.

Im Jahr 2013 bietet Bouvier Rybolowlew das letzte gemalte, noch im Privatbesitz verbleibende Gemälde 
von Leonardo da Vinci (1452-1519) an: “Salvator Mundi», Öl auf Holz, um 1500 (auch bekannt als die männliche Mona Lisa). Rybolowlew ist an einem Kauf interessiert, und das Gemälde wird in sein Loft nach New York transportiert. Bouvier soll mit dem Besitzer einen Kaufbetrag von 80 Millionen US-Dollar ausgehandelt haben, verrechnet Rybolowlew jedoch 127,5 Millionen US-Dollar. Bei einer Silvesterparty 2014 trifft Rybolowlew auf einen New Yorker Kunstberater. Sie kommen auf den Wert eines Modiglianis zu sprechen, welchen Rybolowlew drei Jahre zuvor über Bouvier gekauft hatte. Rybolowlew wird misstrauisch, denn bereits Jahre zuvor wollte er mit der Hilfe von Bouvier - aufgrund der teuren Scheidung von seiner Frau Elena - Teile seiner Kunstsammlung verkaufen, was jedoch laut Bouvier weder mit Gewinn noch zum ursprünglichen Einkaufspreis möglich gewesen wäre. Seine Befürchtungen bestätigen sich: Bouvier hat ihm Kunstwerke mit Aufschlägen von teilweise bis zu 50 Prozent verkauft. Im Fall des Bilds “ Salvator Mundi » gingen knapp 50 Millionen US-Dollar an Bouvier. Im folgenden Rechtsstreit geht es um die Rolle von Bouvier, ob dieser als Verkäufer oder Händler aufgetreten ist. Bouvier wird wegen Betrugs und Geldwäscherei in Höhe von 1.049.465.009 US-Dollar angezeigt, kommt aber gegen Kaution frei. Rybolowlew verklagt Bouvier in Singapur und Hongkong und erreicht das Einfrieren seiner Vermögenswerte; diese werden jedoch wieder freigegeben, das laufende Verfahren wird eingestellt.

Angeblich soll Leonardo da Vinci das Werk um etwa 1500 im Auftrag von Anne de Bretagne und Ludwig XII., dem König von Frankreich, geschaffen haben. Durch die Heirat von Henrietta Maria von Frankreich und König Charles I. von England soll das Bild dann um 1625 nach Greenwich gekommen sein. Ob die Belege hierfür echt sind, ist höchst ungewiss und wird von Experten_innen stark angezweifelt. Zu lückenhaft ist die Provenienz. In den kommenden 150 Jahren wechselt das Gemälde öfter den Besitzer, bis es 1763 in London unter dem Namen "L. Da. Vinci A head of our Saviour» für zwei Pfund versteigert wird. Darauf wechselt es erneut unter verschiedenen Namen den Besitzer. Am 25. Juni 1958 wird es bei Sotheby's London als Werk des italienischen Malers Boltraffio, einem Schüler Leonardos, angeboten und für 45 Pfund Sterling von Warren E. Kuntz, dem Besitzer eines Möbelgeschäfts und Hobby-Kunstsammler aus New Orleans, erworben und als Souvenir von seiner Englandreise als Geschenk für seine Frau mitgebracht. Nach deren Tod im Jahr 1987 wird das Bild an ihren Neffen Basil Clovis Hendry Sr. vererbt. Dieser verkauft den “Salvator Mundi » 2005 für weniger als 10000 US-Dollar an den Kunsthändler Robert Simon. Simon ist auf die Alten Meister spezialisiert und stellt Nachforschungen zu 
dem Gemälde an. Mithilfe der Restauratorin Dianne Modestini und weiteren Experten_innen soll er angeblich die Autorschaft Leonardo da Vincis nachweisen können, die 2011 bekannt gegeben wird. Die Authentizität des Gemäldes wird weiterhin stark angezweifelt, was den Verkauf erschwert. Im Jahre 2013 kommt das Bild dann in den Besitz des russischen Oligarchen Dimitri Rybolowlew.

Im November 2017 soll das Werk von dem saudischen Prinzen Badr Bin Abdullah im Auftrag des Ministeriums für Kultur und Tourismus Abu Dhabi für 450 Millionen US-Dollar bei Christie's New York ersteigert worden sein. Durch diesen Verkauf hätte Rybolowlew einen Gewinn von 270 Millionen US-Dollar erzielt. Vier Tage vor der für Aufsehen sorgenden Auktion eröffnet am 11. November 2017 das Louvre Abu Dhabi. Hier soll “Salvator Mundi” als Gegenstück zur "Mona Lisa” im Louvre Paris präsentiert werden. Auf Twitter teilt das Museum jedoch mit, dass die angekündigte Präsentation vom 18. September bis auf Weiteres verschoben wird. Bis heute werden der letzte Transfer, die Provenienz sowie die Autorschaft des Bilds stark angezweifelt.

\section{Modigliani und die Panama-Papers}

“Homme assis (appuyé sur une canne)» (Öl auf Leinwand) von Amedeo Modigliani (1884-1920) entstand vermutlich 1918 in Paris, wohin Modigliani geflohen war. Modigliani soll das Bild an Roger Dutilleul, einem befreundeten Industriellen und Kunstsammler verkauft haben. Später gelangt es in den Besitz von Oscar Stettiner, einem jüdischen in Paris lebenden Kunsthändler. Während der Besatzung wird das Bild beschlagnahmt und zwangsversteigert und gelangt so im Juni 1944 für 16000 Francs wahrscheinlich zu einem Mann namens Van der Klip. Nach seiner Rückkehr aus dem Exil verlangt Stettiner das Bild von Van der Klip zurück, der aber behauptet, es verkauft zu haben. Noch bevor es zu einem Prozess kommt, verstirbt Stettiner. Im Juni 1996 taucht das Gemälde wieder in der Öffentlichkeit auf und wird anonym bei Christie's zum Verkauf angeboten, auch der Käufer bleibt anonym. Nach dem Verkauf verschwindet das Bild erneut aus der Öffentlichkeit. Die rechtmässigen Erben reichen Klage ein. Zwei Klagen werden abgewiesen, da die Besitzverhältnisse unklar sind. Eine weitere Klage wird eingestellt, nachdem dem Beklagten, der Briefkastenfirma International Art Center S.A., keine Unterlagen zugestellt werden können. 1999 ist das Gemälde bei einer Ausstellung im Museo d'Arte Moderna in Lugano zu sehen. Zwischen 2004 und 2005 wird es in New York ausgestellt, unter anderem in der Helly Nahmad Gallery (welche der Nahmad- 


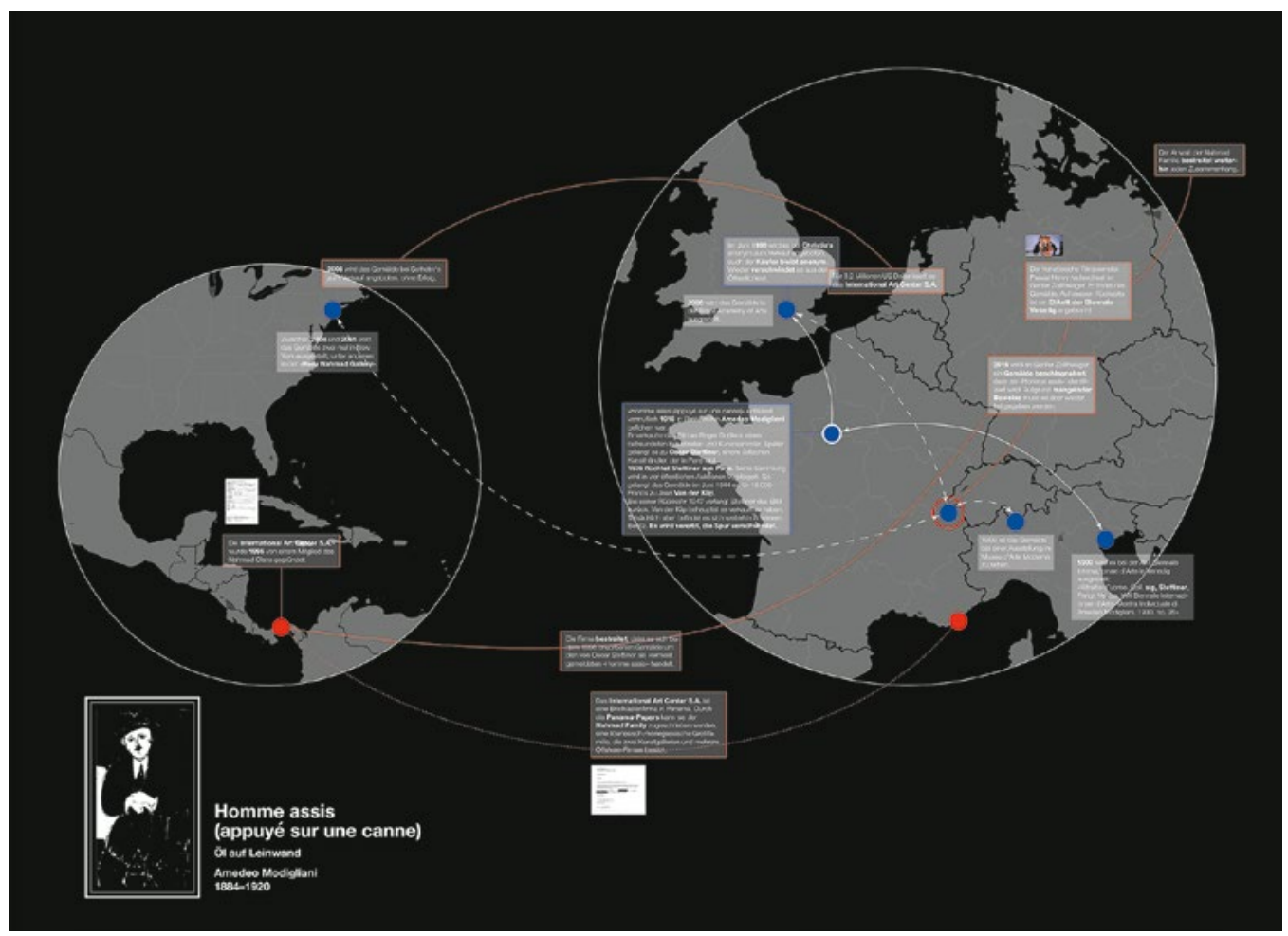

Familie, einer libanesisch-monegassischen Grossfamilie gehört) und 2006 in der Royal Academy of Arts in LonEine visualisierte Provenienzforschung bringt Transparenz in die Zusammenhänge der Besitzverhältnisse des Gemäldes «Homme assis» (1918) don. 2008 wird es bei Sotheby's zum von Amedeo Modigliani.

Verkauf angeboten, jedoch ohne Erfolg. (Abb.66)

Infolge eines Datenlecks gelangen 2016 vertrauliche Unterlagen des Offshore-Dienstleisters Moussack Fonseca an die Öffentlichkeit. Dadurch wird bekannt, was schon lange vermutet worden ist, aber bisher nicht bewiesen werden konnte: Das International Art Center S. A. gehört der NahmadFamilie, einer bedeutenden Kunstsammler-Familie, die das Bild für 3,2 Millionen US-Dollar gekauft haben soll. Die Briefkastenfirma, deren Spur auch in das Genfer Zollfreilager führt, soll zur Verschleierung der Besitzverhältnisse verwendet worden sein. Das International Art Center S. A. wur- 
de 1995 durch die Nahmad-Familie, die familieneigene Kunstgalerien und mehrere Offshore-Firmen besitzt, gegründet. Die Anwälte der Nahmad-Familie bestreiten, dass es sich bei dem von der Briefkastenfirma erworbenen Gemälde um den von Oscar Stettiner als vermisst gemeldeten "Homme assis» handelt, da ein entsprechender Kaufbeleg fehlt. Dem International Consortium of Investigative Journalists (ICIJ) gelingt es jedoch, die Verbindung nachzuweisen. Der französische Filmjournalist Pascal Henry beginnt im Genfer Zollfreilager zu recherchieren. Er kann das Gemälde ausfindig machen, auf dessen Rückseite ein Etikett der Biennale Venedig mit der Aufschrift «Ritratto d'uomo. Coll.sig, Stettiner, Parigi; Venice, XVII Biennale Internazionale d'Arte, Mostra Individuale di Amedeo Modigliani, 1930, no. 35 " angebracht ist. Die rechtmässigen Besitzverhältnisse sind somit bewiesen; die Anwälte der Nahmad-Familie bestreiten jedoch den Zusammenhang.

\section{Der Fall Schwarzenbach}

Der Schweizer Kunstsammler und Devisenhändler Urs E. Schwarzenbach soll jahrelang Kunstwerke und Objekte unversteuert in die Schweiz eingeführt haben. Als Grund wird er später angeben, dass die Einfuhrprozedur am Zoll zu lange dauert und er hierfür schlichtweg keine Zeit habe. Auf die Schliche kommen ihm die Schweizer Zöllner bei einer zufälligen Stichprobe am Flughafen Zürich im Jahre 2012. In seinem Gepäck finden sie zwei unversteuerte Wertsachen: ein Bild von Edwin Long im Wert von über 300000 Schweizer Franken und eine antike Dose im Wert von 11300 Schweizer Franken. Die Oberzolldirektion leitete ein Strafverfahren gegen Schwarzenbach ein. Im April 2013 wird bei Hausdurchsuchungen in Zürich, Küsnacht und St. Moritz belastendes Material gefunden (u. a. eine Datenbank mit einem Verzeichnis der Kunstwerke). Die Oberzolldirektion wirft Schwarzenbach vor, seit dem 23. März 2009 insgesamt 123 Kunstgegenstände unverzollt sowie 27 Objekte mit zu geringen Wertangaben in die Schweiz eingeführt zu haben. In 110 Delikten wird er für schuldig befunden, ohne Verjährungsfrist wären es weit mehr. Die Eidgenössische Zollverwaltung fordert von Schwarzenbach eine Millionenbusse sowie eine Mehrwertsteuernachzahlung im zweistelligen Millionenbereich.

Am 4. Oktober 2018 wird Schwarzenbach zusätzlich mit einer Nachsteuerungsforderung von 270 Millionen Schweizer Franken konfrontiert. Schwarzenbach, der zwischen 2005 und 2012 in England lebte, wird vorgeworfen, den Kunsthandel, welcher er von seiner Villa Falkenstein geführt hat, verschleiert zu haben. Mithilfe von 
Offshore-Firmen - grösstenteils der Galerie Minerva in Liberia - habe er in der Schweiz unversteuerten Kunsthandel betrieben. Schwarzenbach und die Galerie Gmurzynska, deren Besitzer er ist, wird vorgeworfen, mit dem sogenannten Verlagerungsverfahren die Importsteuer auf seine eigene Kunstsammlung umgangen zu haben. Eine Galerie hat die Einführung von Bildern erst in der Steuererklärung auszuweisen, Schwarzenbach als Privatperson hätten dies jedoch bereits bei der Einfuhr deklarieren müssen. Schwarzenbach meint dazu, dass die Bilder von seiner Galerie ausgestellt sind und die Mehrwertsteuer erst bei einem Verkauf der Bilder anfallen würde. Die rund 45 Kunstwerke im Wert von circa 75 Millionen Schweizer Franken, gedacht zur Verschönerung des Hotels Dolder Grand in Zürich, das ebenfalls im Besitz von Schwarzenbach ist, werden jedoch ungeschickterweise auf der Website als Privatsammlung aufgeführt. Belastend für Schwarzenbach ist auch, dass Anwohner_innen verdächtige Helikopterflüge und Warenumschläge gemeldet haben. Mehrmals wöchentlich flöge ein Helikopter der Swiss Jet AG den Landeplatz nahe seinem Reitsportzentrum in Rüti an. Häufig würden Kunstgegenstände verladen und per Limousine oder Kleinbus in die Tiefgarage gefahren. In einer für Aufsehen erregenden Aktion werden die Bilder im Hotel Dolder Grand von der Steuerverwaltung abgehängt und konfisziert. 



\section{Die Applikation Augmented Space}

Die Augmented-Reality-Applikation Augmented Space bietet eine historische Perspektive auf den

Campus der Künste und kombiniert diesen Blick mit einer zeitgenössischen Kritik an der Praxis heutiger Zollfreilager mit Kunstbezug. Die Applikation ermöglicht zwei verschiedene Zugänge: einerseits über Augmentierungen vor Ort (AR und Kompass auf dem Areal), anderseits über Karte, Globus und Modell, welche auch ortsunabhängig angeschaut werden können. Der raumzeitliche Eingriff ist eine Kritik an den monströsen Ökonomien des Kunstmarkts sowie den Praktiken globaler Wirtschaftsnetzwerke beziehungsweise dem bewussten Entzug von Waren aus ökonomischen Zyklen, Netzwerken und Märkten. In 
einer kritisch-politischen Perspektive verdichten sich dynamische Daten und Informationen mit dem historischen Ort und machen den Standort zu einem mehrschichtig erlebbaren Raum. An neun Stationen werden Augmentierungen der historischen Situationen erlebbar: beispielsweise die Autoverladung durch einen elektrischen Kran oder der Lastwagenverkehr auf dem Inlandparkplatz.

Eine spekulative Perspektive gibt Einblick in die Praxis der Zollfreilager mit Kunstbezug. Mit der Applikation wird eine AugmentedReality-Anwendung erprobt und neue Designstandards in Bezug auf Navigation, Interaktion und Informationsvermittlung werden ausgelotet. 

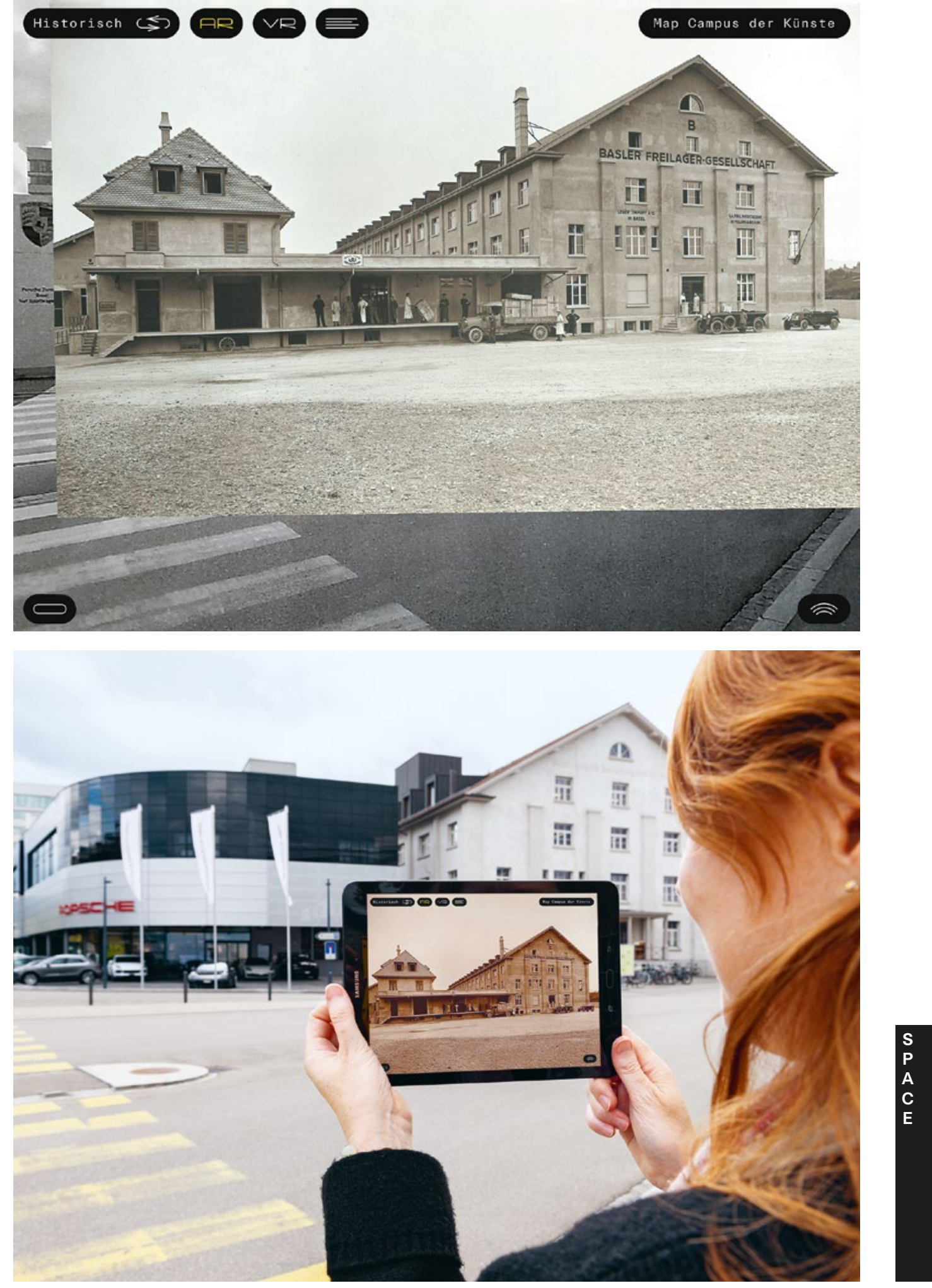

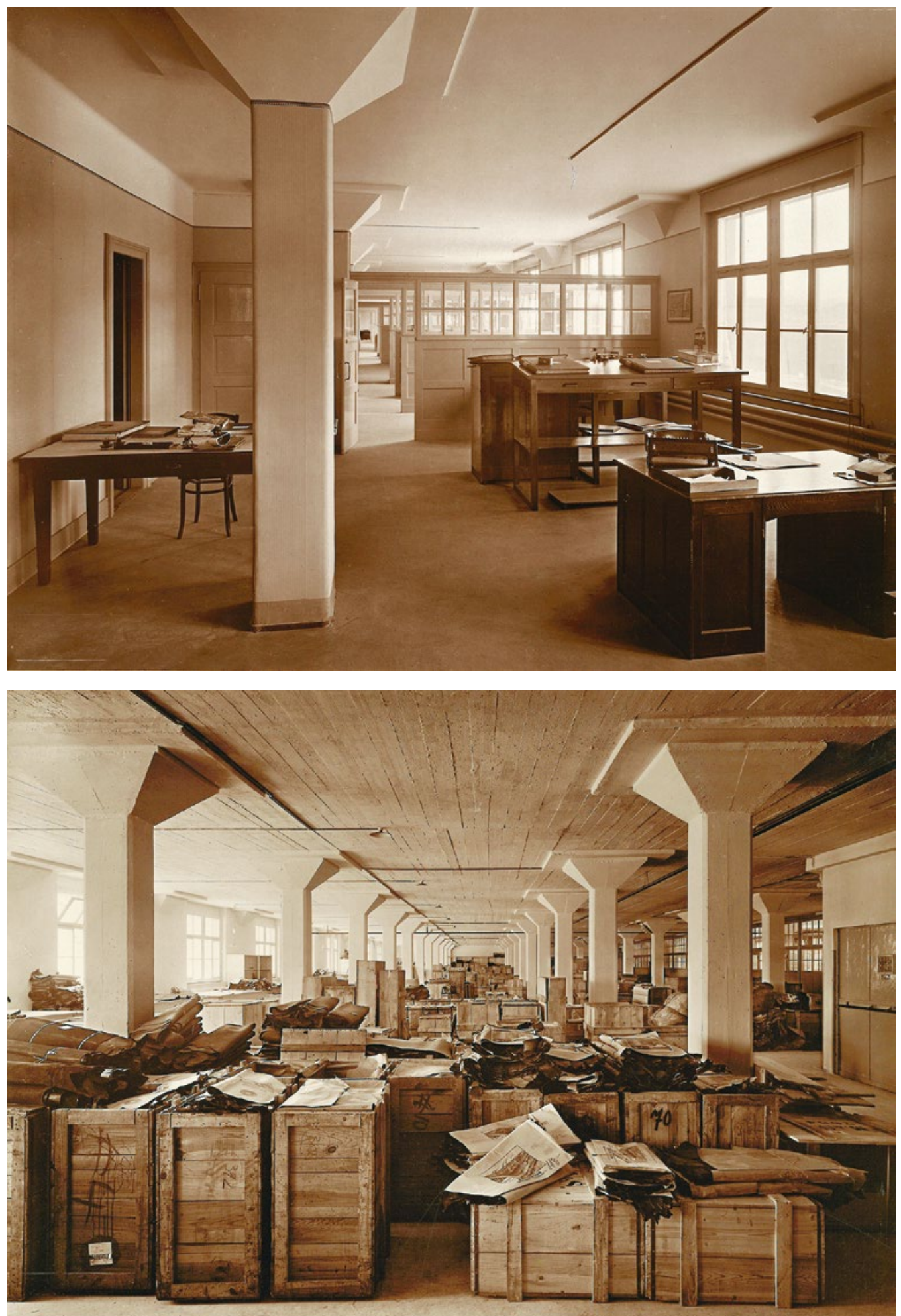


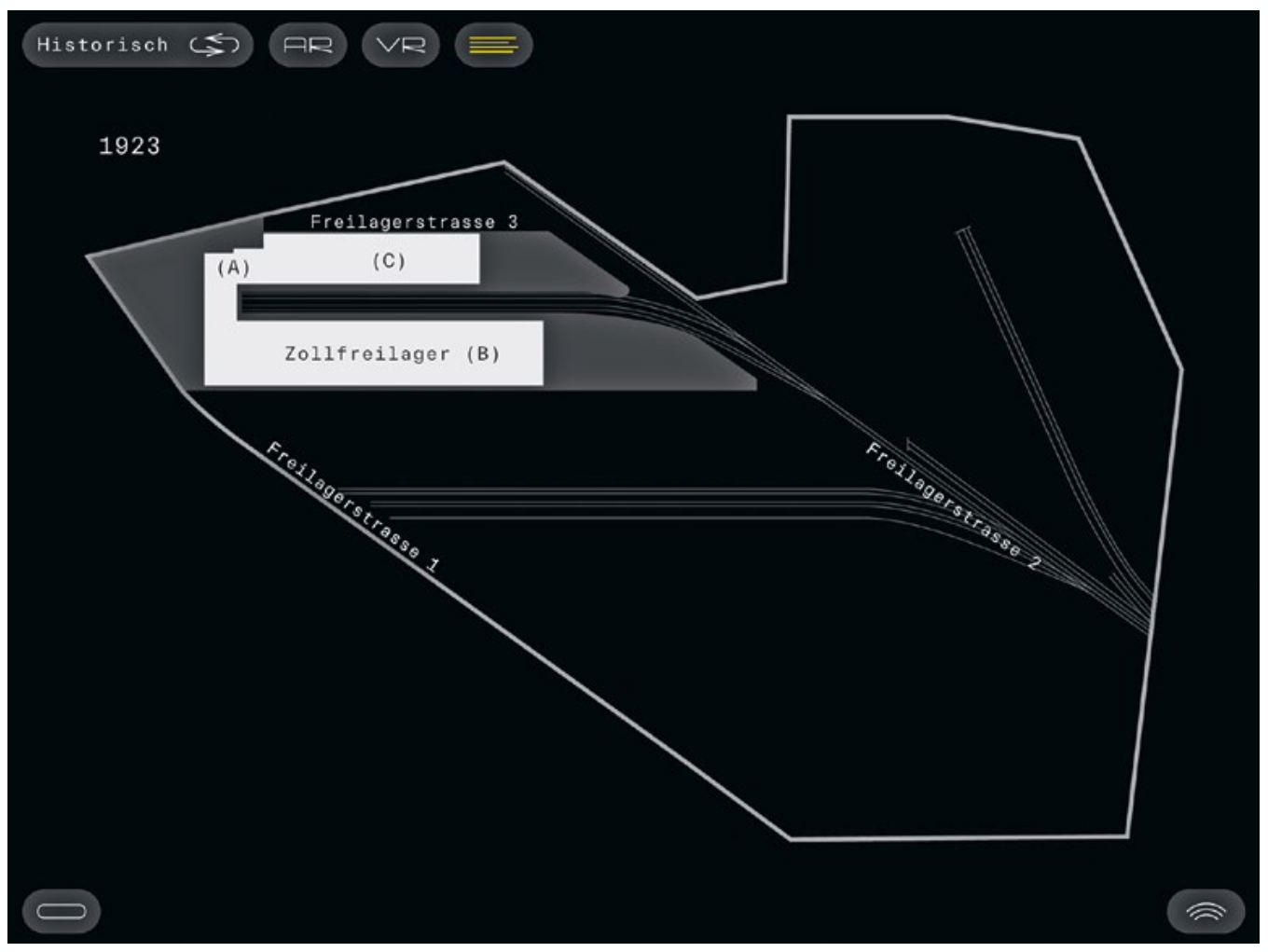

Historisch $\leftrightarrows$ (PR VP

1923

Um den wachsenden Waren und Güterstrom zu bewältigen, fasst der Bundesrat um 1920 den Beschluss, auf dem Areal ein zollfreies Lagerhaus zu errichten. 1922 erfolgt die Gründung der Basler Freilager-Gesellschaft, welche ab 1923 ihren Betrieb aufnimmt. Zur Errichtung des Freilagers stellt die Christoph Merian Stiftung eine Parzelle von rund $50.000 \mathrm{~m}^{2}$ im Baurecht zur Verfügung. Die Architekten Widmer, Erlacher \& Calini werden mit dem Bau des Zollfreilagers, einem Lagerhaus sowie einem separaten Gebäude für die Zollverwaltung mit einer Zollstation beauftragt. Die Zollverwaltung umfasst neben Büros und einem Wachlokal auch Wohnungen für die Zollbeamten. Im neu erstellten Zollfreilager wird fast der ganze 1 . Stock an die Leder-Import AG vermietet. In dieser ersten Bauetappe sind rund $29.000 \mathrm{~m}^{2}$ des Areals genutzt.

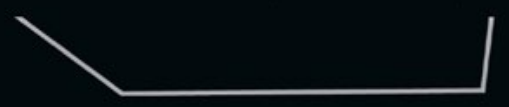


Abb. 73

Abb. 74

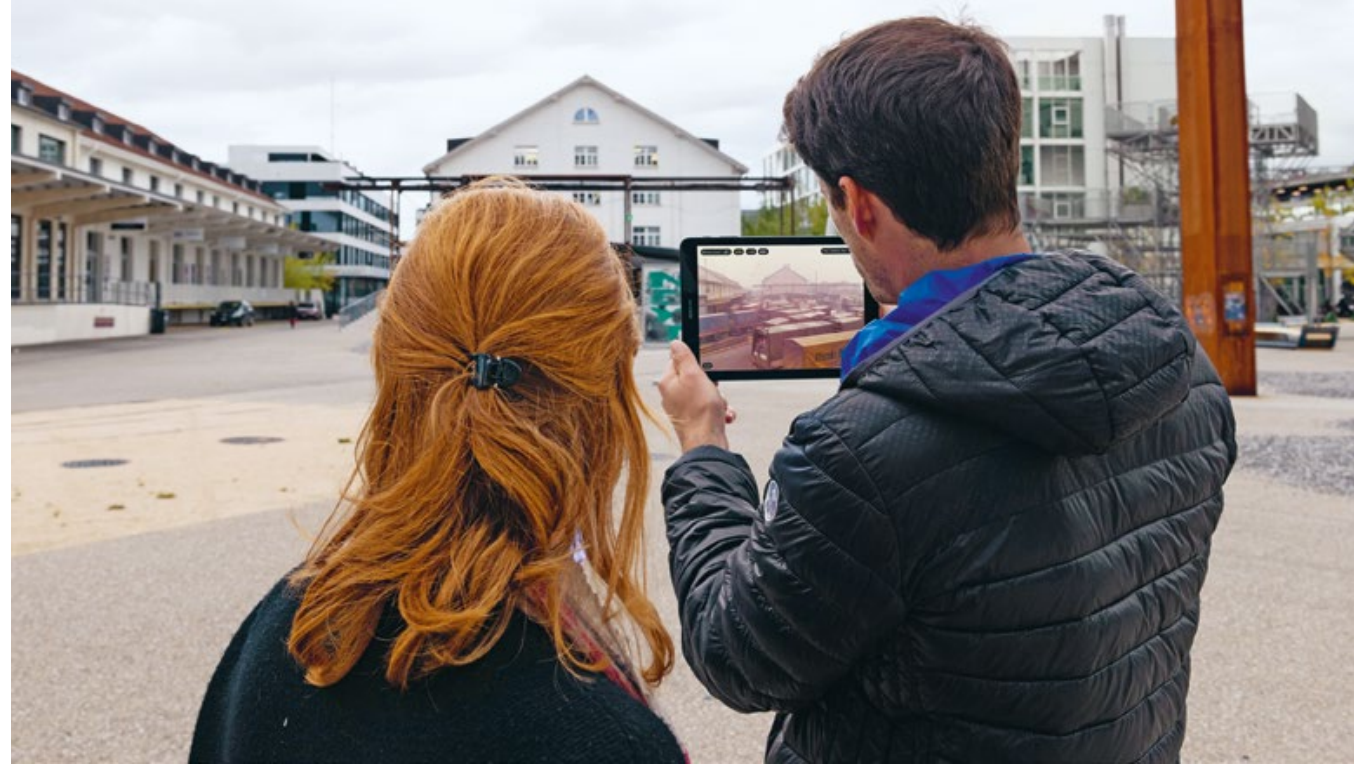

A
$\mathbf{U}$
$\mathbf{G}$
$\mathrm{M}$
$\mathbf{E}$
$\mathbf{N}$
$\mathbf{T}$
$\mathbf{E}$
$\mathrm{D}$

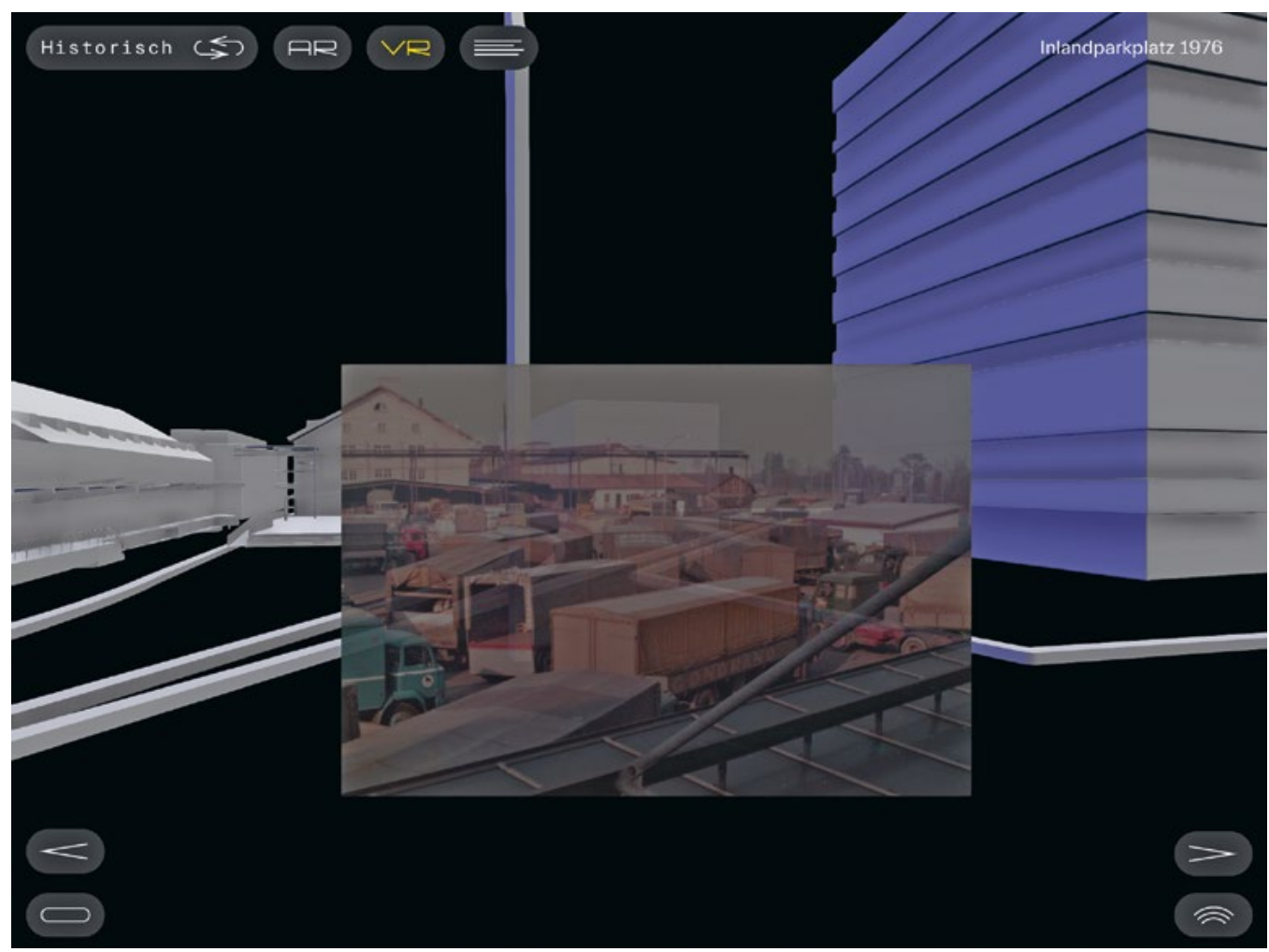




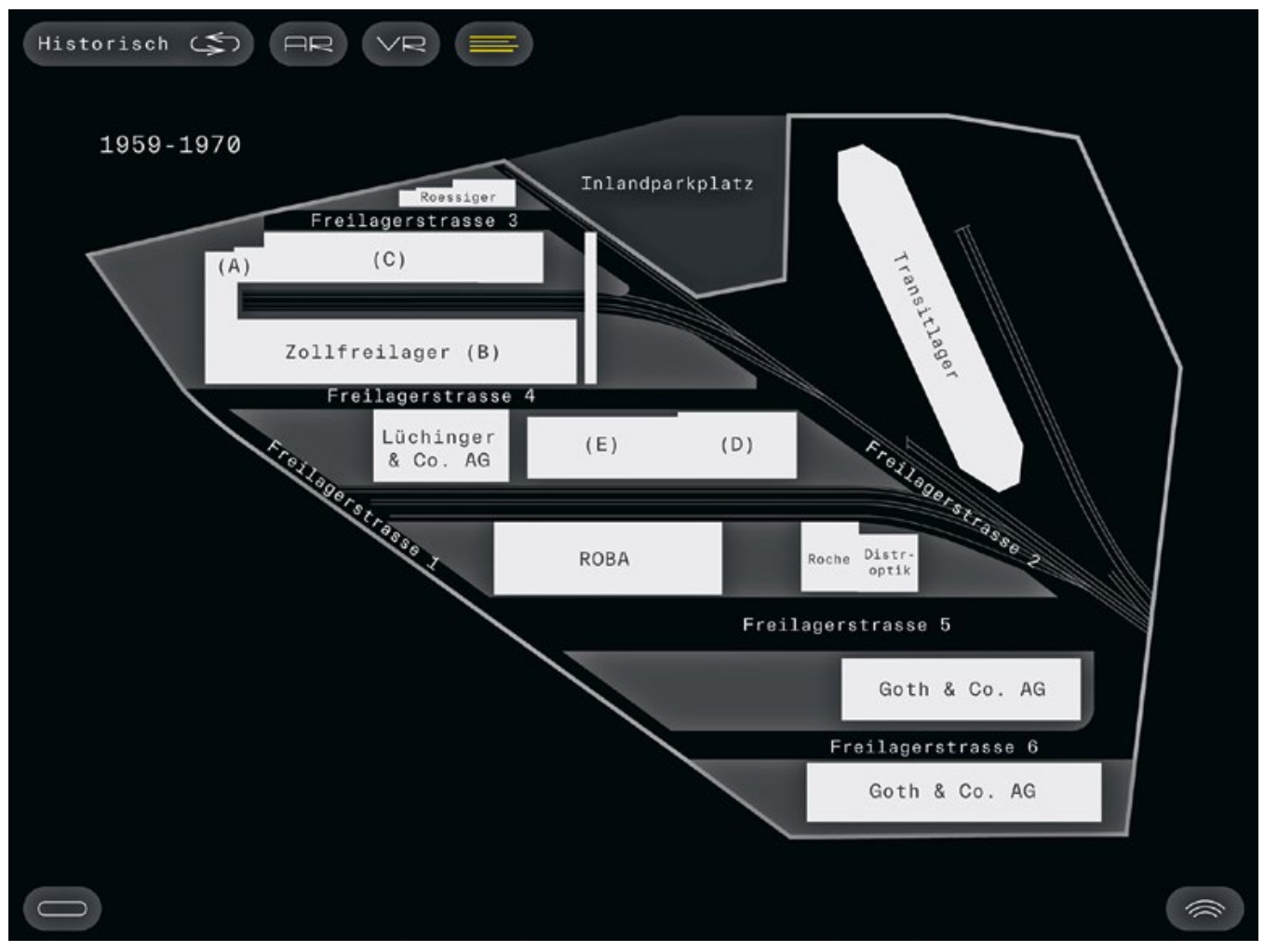

Historisch $\leftrightarrows$ AP VP

1959-1970

Auf der Baureserve entsteht der neue Inlandparkplatz mit angrenzendem Zol Inlandparkplatz Transitlagers dur m

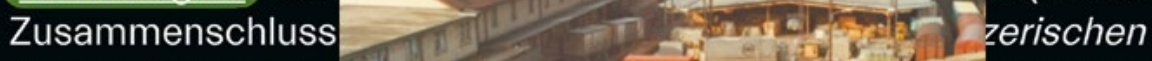
Bundesbahnen sov is äude umfasst fünf Stockwerke u Verträge im Unterb $A G$ (1963), die Bas. AG(1970). $\mathrm{m}^{2}$. Weitere tic AG und Roba e an die Natural
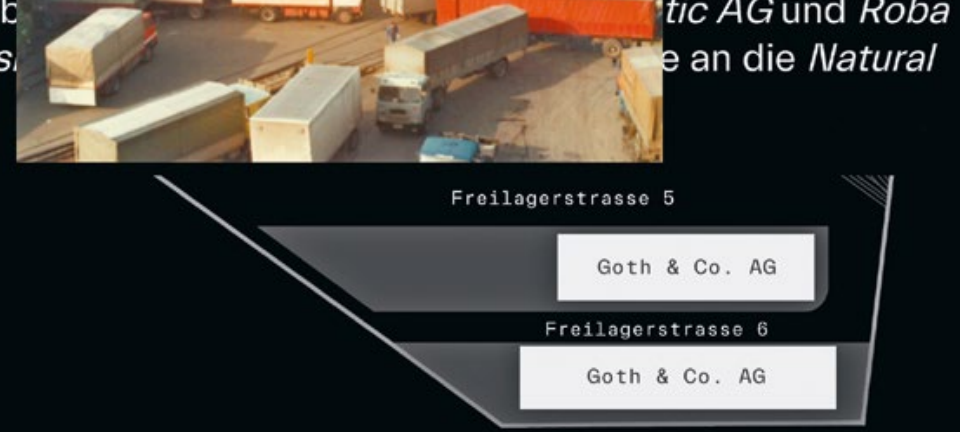

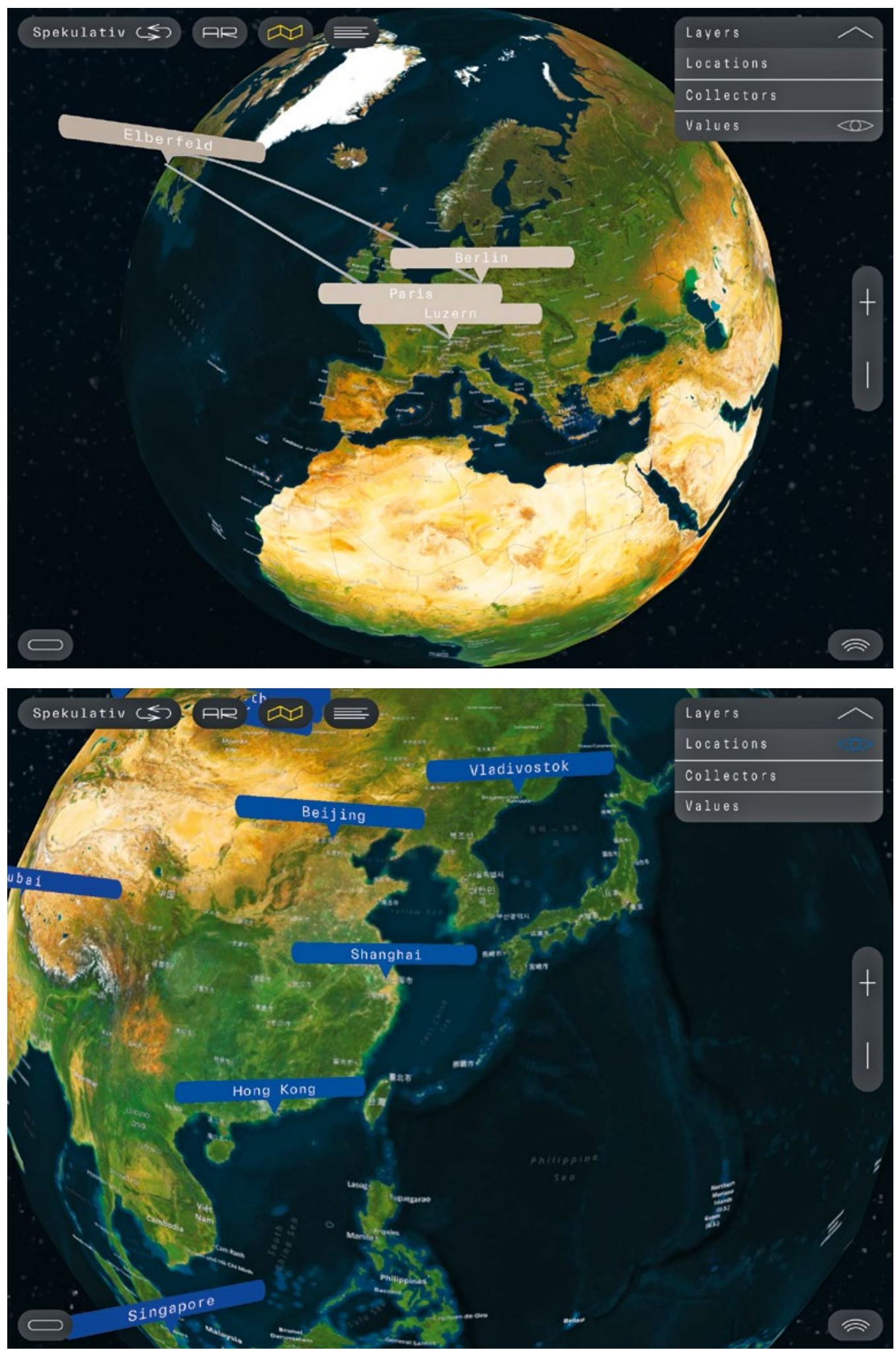

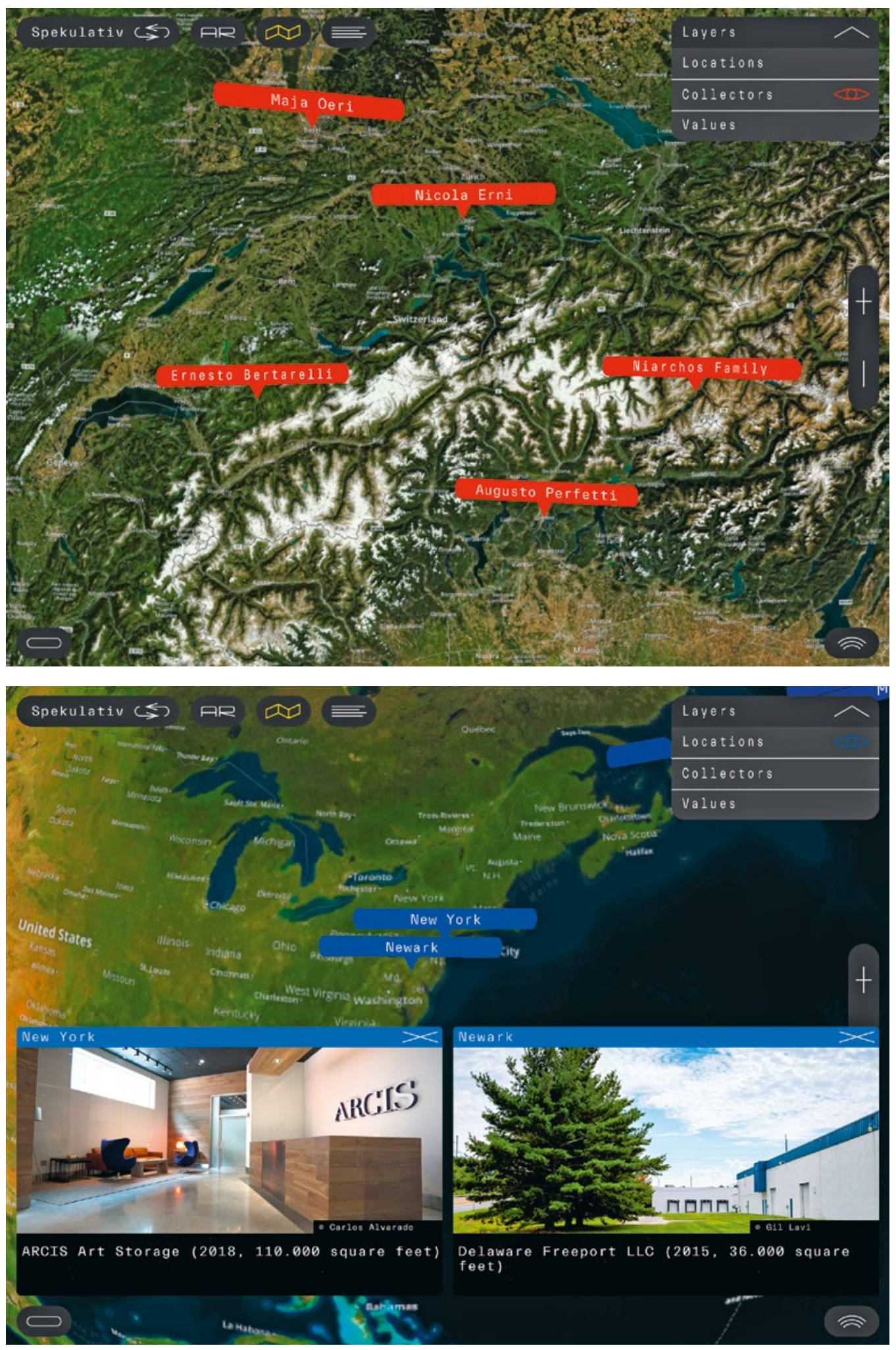
Spekulativ $\leftrightarrows$ (PR D

Stories

Die Bouvier-Rybolowlew-Affăre

Nach über zehn Jahren der Zusammenarbeit verklagt der russische Oligarch Dimitri Rybolowlew den Schweizer Spediteur Yves Bouvier wegen Betrugs. Zentrale Rollen spielen die Freundschaft der beiden Männer, ein wieder entdecktes Gemälde von Leonardo da Vinci sowie eine Milliarde US-Dollar. Das Verfahren wird vor Gerichten in der Schweiz, in Monaco, Frankreich, den Vereinigten Staaten und Singapur ausgetragen.

Yves Bouvier

* 1963 in Genf, ein schweizerischer Unternehmer und

Kunsthăndler.

Bis 2017 ist er Inhaber des Logistikunternehmens Natural Le

Coultre S.A., welches auf Lagerung und Transport von

Kunstwerken spezialisiert ist. Die Firma betreibt

Zollfreilager in Genf, Luxemburg und Singapur.

Dmitri Rybolowlew

*1966 in Perm, ein russischer Unternehmer und Sportsponsor.

Das Vermögen des russischen 0ligarchen wird auf 6.8 Milliarden

US-Dollar geschătzt. 2008 fällt die Aktie seiner Firma

Uralkali massiv, im gleichen Jahr beginnt ein mehrjăhriger

Scheidungsprozess von seiner Frau Elena. Er investiert

vermehrt in Immobilien und Kunst (im Wert von etwa 700

Millionen US-Dollar).

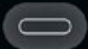

Spekulativ $\leqq$ (日P)

Stories

Unternehmen Natural Le Coultre S.A. zu verkaufen. Das laufende Verfahren gegen Bouvier wird eingestellt. $\rightleftharpoons$

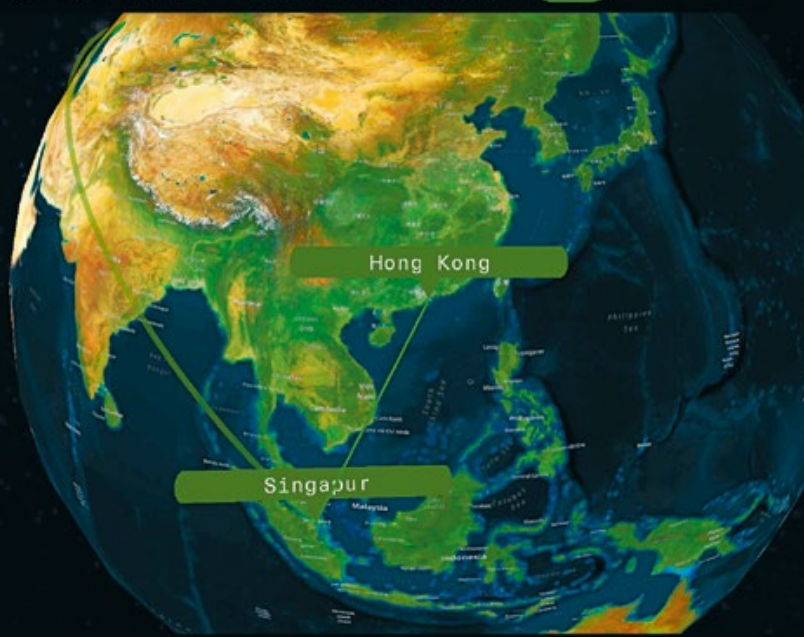

Im November 2017 wird Salvator Mundi von dem saudischen Prinz Badr 


\section{Geschlechterverteilung im Kunstmarkt}

Unter den 50 gefragtesten Malern auf dem Kunstmarkt befindet sich laut Kunstindex (Manager Magazin, 2018) gegenwärtig keine einzige Frau. Unterscheidet man jedoch die erzielten Preise am Auktionsmarkt von der Anzahl der Verkäufe, werden auch Künstlerinnen sichtbar. Die Gender Pay Gap ist im Kunstmarkt Alltag. Umso mehr erstaunt, dass mehr als die Hälfte der Künsthändlerinnen im Top-Segment weiblich ist.

«Ein Bild von Cindy Sherman wurde für über zwei Millionen Dollar verkauft, ihre neuen Arbeiten liegen aber bei 500.000 Dollar. Da fängt Jeff Koons erst an. Ist Jeff Koons bedeutender als Cindy Sherman?»

Top 20 Artists for Works Created in the Last 20 Years

Top 20 Selling Living Artists

Top Prices for Living Artists

Top 20 Selling Post War and Contemporary Artists

Top Prices in the Post War and Contemporary Sector

Top Selling Artists in the Modern

Top Prices in the Modern Sector

Top 20 Selling Impressionist and Post Impressionist Artists

Top Prices in the Impressionist and Post Impressionist Sector

Top 20 Selling Old Master Artists 

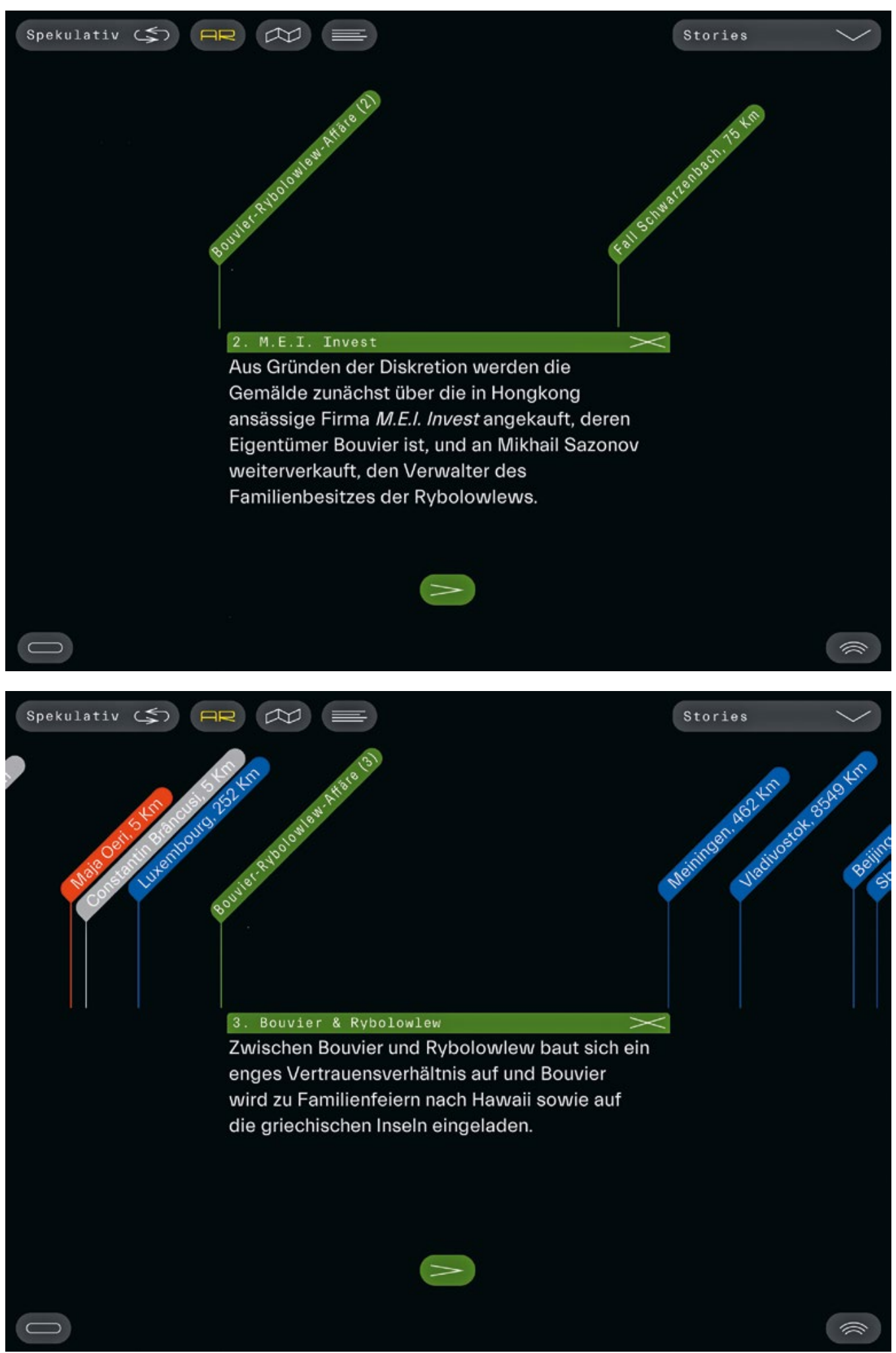
Abbildung 67-68:

Unterwegs auf dem Rundgang mit AR-Funktion, hier die Augmentierung des Zollgebäudes auf der Applikation

Abbildung 69-72:

Unter Text können weitere Informationen zu den augmentierten Standorten abgerufen werden, hier zur Leder-Import AG im ersten Stock der Zollverwaltung.

Abbildung 73-76:

Alle drei zur Auswahl stehenden Darstellungsmodi der historischen Perspektive AR, VR (virtuelle Tour) sowie Text

Abbildung 77-80:

In der spekulativen Perspektive können in den Modi Karte bzw. Globus verschiedene Elemente angezeigt werden, hier Locations, Collectors und Values.

Abbildung 81-84:

Unter Text werden skandalöse Geschichten im Zusammenhang mit dem Kunstmarkt erfahrbar (Bouvier-Rybolowlew-Affäre, Fall Schwarzenbach, Modigliani und die Panama-Papiere sowie der Gender Pay Gap).

Abbildung 85-86:

Im Erzählmodus werden über eine Kompassfunktion die verschiedenen Elemente sowie die Geschichten in den Raum augmentiert. 


\section{Die historische Ebene}

Um die historischen Bilder verfügbar zu machen, wurde eine Augmented-Reality-Applikation entwickelt. Augmented Reality bietet die Möglichkeit, mit einer einfachen Technologie komplexe Informationen leicht verständlich darzustellen und mit dem physischen Raum in Beziehung zu setzen. Die Informationsarchitektur der Applikation ermöglicht verschiedene Lese- und Sichtweisen auf das Areal. Beim Einstieg in die App kann der Nutzer zwischen einer historischen beziehungsweise spekulativen Ebene wählen. Die Auswahl kann jederzeit gewechselt werden. Sowohl auf der historischen wie auch der spekulativen Ebene bietet die Applikation verschiedene Darstellungs- und Erzählmodi. Bei der historischen Ebene stehen drei Modi zur Verfügung: AR, VR sowie Text. Der Modus AR ermöglicht als $A R$-Rundgang an neun Stationen einen Eindruck der früheren Nutzung als Zollfreilager. An den jeweiligen Standorten werden mittels Augmentierungen historische Aufnahmen verfügbar gemacht, welche den Alltag des Zollfreilagers erleben lassen. Mit einem Klick auf das Bild wird die Ansicht gedreht, und anstelle des Bilds erscheinen weiterführende Informationen zum Gebäude beziehungsweise zum Objekt. Durch einen erneuten Klick dreht sich die Ansicht wieder auf die Augmentation. Die neun Stationen des AR-Rundgangs sind auf einer Karte verzeichnet und zusätzlich am Boden markiert. Neben dieser Möglichkeit des freien Entdeckens gibt es mittels des Modus VR eine geführte VR-Tour. Die virtuelle Tour verbindet Elemente aus Virtual Reality und Augmented Reality. Anstelle der Überlagerung im Raum erfolgt die Überblendung der historischen Situation auf einem 3-D-Modell des Areals. Die Tour beginnt mit einer Luftaufnahme des Zollfreilagers und führt entlang seiner Entstehungsgeschichte chronologisch aufsteigend durch die Geschichte der früheren Nutzung. Die entsprechenden Szenen werden jeweils angeflogen und mit den historischen Bildern überlagert. Die virtuelle Tour kann unabhängig vom physischen Raum überall erlebt werden. Unter Text werden umfassende Informationen zur Bau- und Nutzungsgeschichte des Areals vermittelt.

Mittels der Applikation können an den markierten Stationen die historischen Bilder über das Kamerabild eingeblendet werden. Sobald das Tablet über das Kamerabild einen entsprechenden Standort erkennt, überlagert sich das Kamerabild und das historische Bild wird angezeigt. Die Überblendung der historischen Bilder ist nur an den exakten Standorten möglich, da es sonst zum Verlust des räumlichen Erlebnisses kommt. Für die Augmentierung wurden die historischen Bilder georeferenziert und im Raum orientiert. Mittels der 
Software Vuforia werden aktuelle Aufnahmen an den jeweiligen Stationen zu Targets umgewandelt und anschliessend in Unity importiert. Vuforia ist eine der führenden AR-Plattform, die unter anderem in Unity 3-D - Game Engine, in welcher die App realisiert worden ist - einfach verwendet werden kann. In Unity werden die Bilder zu den Targets orientiert. Unity ist eine Entwicklungsumgebung für jegliche Plattformen, Smartphones oder Tablets und wird vor allem für Computerspiele verwendet. In dem Editor können 3-D-Objekte angeordnet, texturiert, animiert sowie verschiedenste Funktionalitäten implementiert werden. Mithilfe von C\#-Script kann beispielsweise die Funktion zum Ein- und Ausschalten von Objekten sowie zur Umsetzung der Navigation genutzt werden. Unter den ausgewählten historischen Situationen gibt es auch Innenaufnahmen der Firma Leder-Import AG, die sich im ersten Stock des Zollfreilagers (Gebäude B) befand. Um diese Aufnahmen verfügbar zu machen, werden diese an die Aussenwand des Gebäudes projiziert. Die Projektion wird mittels einer virtuellen Galerie erlebbar. Diese Galerie wird - wie alle historischen Situationen - durch die korrekte Positionierung der Kamera ausgelöst. Die Aufnahmen sind in einem quadratischen Raum an drei Wänden eingeblendet, was das räumlich-immersive Konzept und die User-Experience zusätzlich unterstützt. Das 3-D-Modell für die virtuelle Tour wurde in Zusammenarbeit mit Studierenden des Bachelor-Studiengangs Geomatik erstellt. Zur Erstellung des Modells wurde das Areal zunächst mit Drohnen (Sensefly Ebee und DJI Phantom 4 Pro), einem 3-D-Laserscanner und digitalen Kameras georeferenziert vermessen. Mittels Fotogrammetrie wurden die Daten im Anschluss zusammengetragen und die Rekonstruktion der bestehenden Gebäude und Objekte erarbeitet. Die einzelnen 3-D-Modelle wurden texturiert und für den Einsatz in Virtual-Reality-Anwendungen zusammengesetzt.

\section{Die spekulative Perspektive}

Die historische Ebene wird durch eine spekulative Perspektive erweitert, die das Kunstsammeln, den Kunstmarkt sowie die Zollfreilager mit Kunstbezug thematisiert. Durch diese Gegenüberstellung ermöglicht die App verschiedene Lese- und Sichtweisen auf das Areal. In einer kritisch-politischen Dimension verdichten sich Bilder, Information und Echtzeit-Meldungen zu einem spekulativen Raum. Bei der spekulativen Ebene stehen ebenfalls drei Modi zur Verfügung: AR, Globus oder Text. Je nach Modus-Auswahl können die entspre- 
chenden Inhalte im Raum augmentiert oder auf dem Globus angezeigt werden. Die AR-Funktion erfolgt hier in Form einer Kompass-Ausrichtung. Bei der Globus-Funktion werden die verfügbar gemachten Informationen auf einem stufenlos skalierbaren Globus dargestellt, welcher sich ab einer gewissen Zoomstufe in eine Karte (Mapbox Satellite) verwandelt. Die Inhalte setzen sich aus mehreren Layern zusammen (Locations, Collectors, Values, Stories), die die Praxis um die Kunstfreilager näher vermitteln. Locations verortet die Standorte der sieben Zollfreilager in der Schweiz sowie die elf globalen Zollfreilager mit Kunstbezug. Neben dem Standort werden auch Bilder der Gebäude sowie weiterführende Informationen verfügbar gemacht. Collectors gibt einen Einblick in das Thema Kunstsammeln und zeigt, welche bedeutenden Kunstsammler_innen ihren Wohnsitz in der Schweiz haben. Values gibt einen Überblick über die weltweit teuersten Kunstwerke (Nachkriegs- und zeitgenössische Kunst) und deren Verbleib. Auf Grundlage von Provenienzforschung wird ersichtlich, wie sie zu ihren jetzigen Besitzer_innen gekommen sind und wann sie zuletzt öffentlich zu sehen waren. Unter Stories werden vier Geschichten erzählt, die alle mit der Schweiz in Verbindung stehen und in die Welt der Kunstskandale einführen, wie beispielsweise die Geschichte des Kunstsammlers Urs E. Schwarzenbachs, welcher zur Vermeidung von Steuern einzelne Kunstwerke per Helikopter in die Schweiz transportierte.

Alle Inhalte wurden bereits detailliert beschrieben. Die Recherche und Erarbeitung der Informationen war dabei ein wichtiger Teil der Entwicklung und Realisierung der Applikation. Die Inhalte haben jeweils einen örtlichen Bezug, welcher auf dem Globus verortet ist. Zudem wird der Standort des Inhalts mittels Kompassfunktion und Distanzangabe in den Raum augmentiert. Um zu den Inhalten zu navigieren, lässt sich der Globus durch eine Wischbewegung drehen beziehungsweise über eine Multitouch-Zoomfunktion stufenlos sowie über Buttons in Stufen vergrössern und verkleinern. Über eine 360-Grad-Drehbewegung des Tablets lassen sich die Inhalte in exakter Ausrichtung in den Raum augmentieren. Die Layer sind einzeln oder auch kombiniert auswählbar. Eine zusätzliche Ebene bestehend aus einem digitalen Schatten wird über die App gelegt. Unter digitalem Schatten werden hier die Datenspuren verstanden, welche die Sammler_innen im Internet hinterlassen beziehungsweise generieren, indem sie beispielsweise ein Kunstwerk ihrer Kunstsammlung auf Instagram stellen. Dieser digitale Fussabdruck wird anhand von Feeds abgerufen und über APIs verfügbar gemacht. Durch das Zusammentragen der Echtzeit-Daten zeichnet sich eine komplexe Perspektive auf die Thematik. 
Die Umsetzung der spekulativen Ebene erfolgte mit einem digitalen Globus und einer Kompassansicht. Der Globus dient hier einerseits der freien Erkundung der verschiedenen Positionen von Kunstwerken, internationalen Zollfreilagern und den Ausgangspunkten der erzählten Stories. Andererseits bietet er mit den Animationen innerhalb der Stories räumliche Informationen über die erzählten Geschichten und die Provenienzen der Kunstwerke. Eine Schwierigkeit war dabei, die Koordinaten der Inhalte zuerst in das Koordinatensystem von Unity zu überführen und danach über polare Koordinaten auf dem Globus richtig zu platzieren. Die animierten 'Fluglinien, innerhalb der Stories, die korrekten Animationen, die Nachverfolgung der zurückgelegten Wege und die Koordination der ein- und ausschaltenden Verortungen stellten weitere Herausforderungen dar. Ebenso ist eine saubere und User-freundliche Touch-Steuerung nicht zu unterschätzen. Der AR-Teil der spekulativen Ebene wurde mithilfe einer Kompassansicht umgesetzt. Diese zeigt, je nach eingeschalteten Ebenen, alle oder einzelne Inhalte im Raum an. Wird das Tablet gegen den Horizont gerichtet, werden die Inhalte mit Markern in der korrekten Himmelsrichtung relativ zum Campus der Künste angezeigt. Hier musste darauf geachtet werden, dass nicht alle mobilen Geräte die Beschleunigungssensoren und Gyroskope (Kreisel), welche neben dem Magnetometer (Kompass) für die Steuerung verwendet werden, gleich orientiert sind.

\section{Fazit}

In einer beispielhaften Praxis wurde mittels der AR-Applikation ausgelotet, wie mit der Technologie von Augmented Reality historische und aktuelle Informationen nutzbar gemacht werden können. Dabei wurde bewusst das Potenzial in einem hybriden Raum erforscht (in der Durchdringung des digitalen mit dem physischen Raum). Das Ziel war ein besseres Verständnis in Bezug auf Medientechnologie, Nutzbarkeit und Grenzen vom Erleben hybrider Räume mit Fokus auf Interaktion und Informationsdarstellung. Die Erstellung der App beinhaltete das User Interface (Benutzeroberfläche) sowie die User Experience (Interaktion) in beziehungsweise mit der physischen Umgebung (Zollfreilager Basel) und den erweiterten Räumen (aktuelle Praxis der Zollfreilager mit Kunstbezug). Für die Gestaltung der Benutzeroberfläche wurde ein entsprechendes Konzept ausgearbeitet, das einen Zugang zur sowohl materiell-physischen wie auch virtuell-digitalen Welt möglich macht und die Inhalte in einem hybriden 
Raum vermittelt. Dabei ging es einerseits um die Vermittlung von nicht (mehr) real sichtbaren Zuständen, andererseits aber auch um das Zusammentragen, Sichtbarmachen und Kontextualisieren von Daten aus dem Geo-Web. In einem iterativen Prozess wurde eine prototypische Umsetzung eines Augmented-Reality-Interfaces entwickelt. Ein Schwerpunkt war die Erforschung, wie Inhalte nutzerund mediengerecht präsentiert werden können. Ein zweiter Schwerpunkt bildete die Interaktion mit dem Ort sowie die Dramaturgie der Inhalte respektive wie diese erzählt werden und durch diese navigiert wird. Erzählerische Qualitäten und Dramaturgie sowie eine intuitive Navigation unterstützen den Prozess.

Vier Herausforderungen können aus dem Forschungsresultat extrahiert werden:

1. Technisch anspruchsvoll war die genaue Positionierung für die Augmentierungen. Da die Technologie bei der Standortbestimmung mittels GPS-Signal immer noch grosse Ungenauigkeiten aufweist, ist eine Karte mit Standortmarkern nur bedingt brauchbar. Deshalb haben wir uns für zusätzliche Bodenmarkierungen an den AR-Positionen auf dem Campus entschieden.

2. Eine weitere technische Schwierigkeit ist der Akkuverbrauch. Die AR-Technologie verbraucht aufgrund der hohen Nutzung an Gerätesensorik und der ständigen Prozessierung des Kamerabilds Unmengen an Akkulaufzeit, was wohl unter anderem ein Grund dafür sein dürfte, dass der Einsatz von AR noch zurückhaltend erfolgt. Es wird interessant sein zu sehen, wie Google mit dieser Problematik auf Google Maps im Zusammenhang mit der neuen AR-Funktion umgehen wird.

3. Eine Schwierigkeit bezüglich User Experience und User Interface war die komplexe und dichte Informationsvermittlung auf einem kleinen Display. Um Erkenntnisse zu gewinnen, sind wir hier bewusst an die Grenzen des Darstell- und Machbaren gegangen.

4. Eine weitere Schwierigkeit war die Verständlichkeit der neuen Erzählformen (wie z.B. Story mit Kompassfunktion in den Raum augmentiert). Ein Nutzer, dem eine solche Funktion bisher noch nicht begegnet ist, versteht sie wahrscheinlich nicht sofort. 


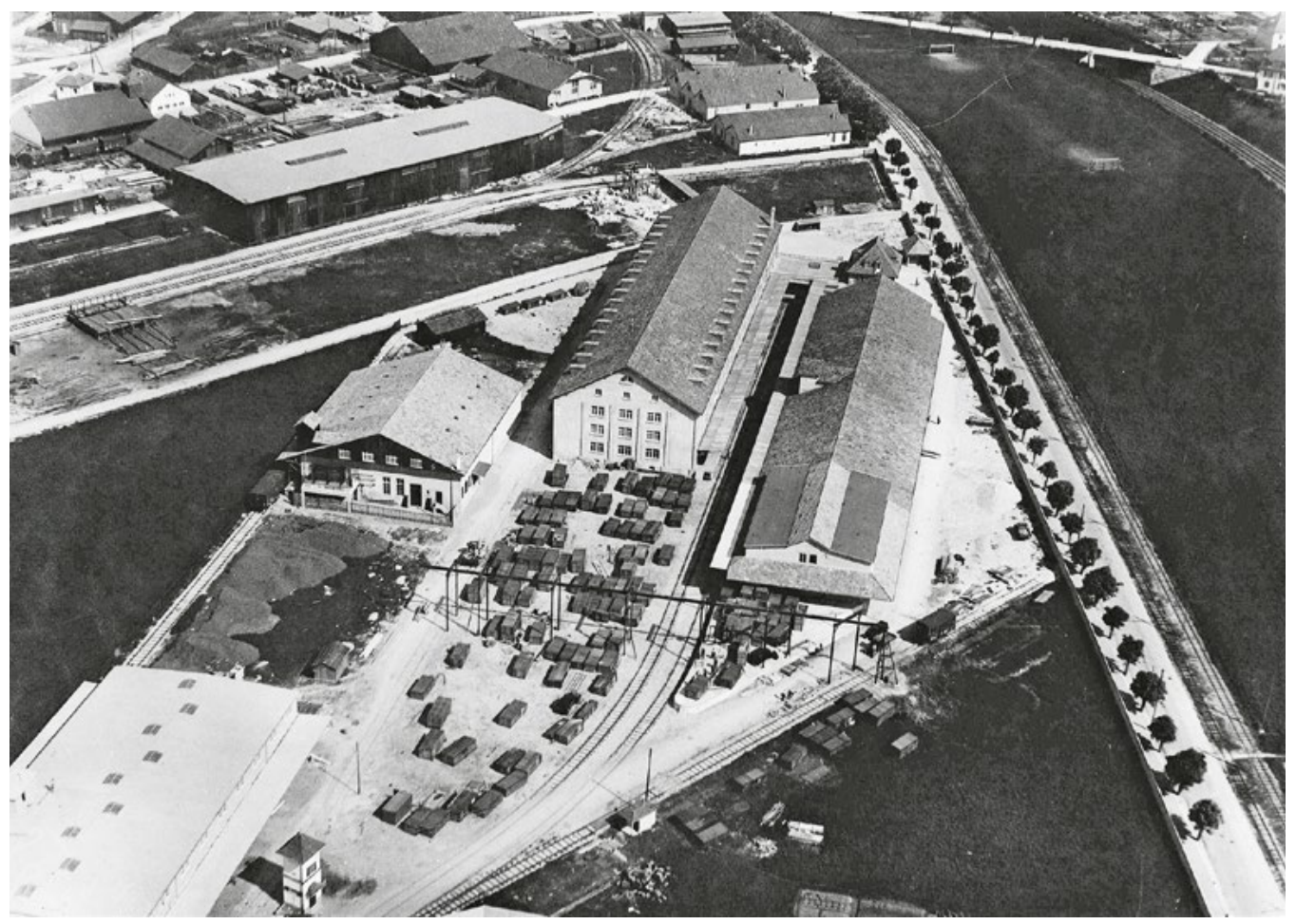

\section{Die App zum Buch}

Die App Augmented Space ermöglicht eine virtuelle Zeitreise. Beginnend mit einer Luftaufnahme über das Zollfreilager führt die Tour durch die Geschichte seiner früheren Nutzung bis ins Jetzt. Die aktuelle Szenerie wird dabei jeweils mit historischen Bildern überlagert und ermöglicht damit ein gleichzeitiges Erleben von Vergangenem und Gegenwärtigem.

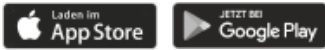

Die App kann im Apple App Store und

bei Google Play Store heruntergeladen werden. 

Anhang 



\section{Kurzbiografie}

Christine Schranz hat Visuelle Kommunikation studiert und in Film-, Medien- und Theaterwissenschaft promoviert. Als Wissenschaftlerin ist sie seit ihrer Promotion in der Designforschung tätig und gilt als profunde Kennerin von Design-Methodologien und entwurfsbasierter Forschung.

Als Designerin hat sie mehr als 20 Jahre internationale Projekte für die Industrie und Wirtschaft geleitet und war hierbei insbesondere für die systematische Entwicklung von Marken und Leitsystemen und deren Implementierung in klassischen sowie digitalen Medien verantwortlich.

Aktuell ist sie Senior Researcherin am Institut Experimentelle Design- und Medienkulturen und Mitglied des Leitungsteams am Institut Integrative Gestaltung | Masterstudio an der Hochschule für Gestaltung und Kunst FHNW, wo sie als Co-Leiterin für das Forschungsprogramm des Instituts und der Masterprogramme verantwortlich ist. In dieser Funktion betreibt sie eine Neuausrichtung des Masterstudiengangs hin zu digitaler Medienkompetenz und vernetztem Gestalten. Ihr Forschungsinteresse gilt dem digitalen, ästhetischen und kulturellen Wandel und dem Einfluss von Design auf die Gesellschaft.

www.christine-schranz.ch

www.ixdm.ch/team/christine-schranz 
Abend, Pablo (2013). Geobrowsing. Google Earth und Co. Nutzungspraktiken einer digitalen Erde. Bielefeld: transcript.

Abernathy, David Ray (2017). Using Geodata \& Geolocation in the Social Sciences. Mapping our Connected World. Los Angeles: SAGE.

Abrams, Janet \& Hall, Peter (2008). Else/where: Mapping New Cartographies of Networks and Territories.

Minneapolis: University of Minnesota.

Adam, Georgina (2017). Dark Side of the Boom. The Excesses of the Art Market in the Twenty-first Century.

London: Lund Humphries.

Akademie der Künste (2005). Topos Raum. Die Aktualität des Raumes in den Künsten der Gegenwart. Hg. v. Angela Lammert u. a. Nürnberg: Verlag für Moderne Kunst.

Apprich, Clemens (2015). Vernetzt Zur Entstehung der Netzwerkgesellschaft. Bielefeld: transcript.

Arnheim, Rudolf (1964). Kunst und Sehen. Eine Psychologie des schöpferischen Auges. Berlin: de Gruyter.

Ash, James/Kitchin, Rob/Leszczynski, Agnieszka (2019). Digital Geographies. London: Sage Publications.

Avanessian, Armen \& Hofmann, Franck (2010). Raum in den Künsten. Konstruktion, Bewegung, Politik. München: Fink.

Bachmann-Medick, Doris (2010). Cultural Turns. Neuorientierungen in den Kulturwissenschaften. Reinbek bei Hamburg: Rowohlt Taschenbuch.

Badura, Jens/Rey, Anton/Dubach, Selma/Haarmann, Anke/Mersch, Dieter/Schenker, Christoph/Toro Pérez, Germán (Hg.) (2015). Künstlerische Forschung. Ein Handbuch.

Zürich: Diaphanes.

Banz, Claudia (2016). Social Design. Gestalten für die Transformation der Gesellschaft (Design 6).

Bielefeld: transcript.

Basler Freilager AG (1947). Basler

Zollfreilager 1922 - 1947.

Basel: Basler Zollfreilager.

Bennahum, David S. (1998). Extra Life. Coming of Age in Cyberspace.

New York: Basic Books.
Bense, Max (1982). Aesthetica.

Einführung in die neue Aesthetik.

Baden-Baden: Agis-Verlag.

Bertin, Jacques (1967). Sémiologie graphique. Paris: Mouton.

Bhorkar, Gaurav (2017). A Survey of Augmented Reality Navigation. www.arxiv.org/pdf/1708.05006.pdf (abgerufen am 25.06.2019).

Bippus, Elke (Hg.) (2012). Kunst des Forschens. Praxis eines ästhetischen Denkens. Schriftenreihe des Instituts für Gegenwartskünste, Zürcher Hochschule der Künste. Zürich: diaphanes.

Bodenhamer, David J./Corrigan, John/Harris, Trevor M. (2015).

Deep Maps and Spatial Narratives

(The Spatial Humanities).

Bloomington: Indiana University Press.

Böhme, Gernot (1995). Atmosphäre.

Essays zur neuen Ästhetik.

Frankfurt am Main: Suhrkamp.

Böhme, Gernot (2006). Architektur und Atmosphäre. München: Wilhelm Fink.

Böhme, Gernot (2016). Ästhetischer Kapitalismus. Berlin: Suhrkamp.

Bonnett, Alastair (2016). Die seltsamsten Orte der Welt. Geheime Städte. Verlorene Räume. Wilde Plätze. Vergessene Inseln. München: C.H.Beck.

Borries, Friedrich von (2017).

Weltentwerfen. Eine politische Designtheorie. Berlin: Suhrkamp.

Brändle, Christian (2014). «Kartografie als Uhrwerk.» In: Ders./Gimmi, Karin/ Junod, Barbara Junod/Richter, Bettina/ Rebele, Christina Rebele (Hg.): 100 Jahre Schweizer Grafik. Zürich: Lars Müller, S. $156-159$.

Bratton, Benjamin H. (2015). The Stack. On Software and Sovereignty.

Cambridge, Mass./London: MIT Press.

Braun, Georg \& Hogenberg, Franz (1965).

Civitates Orbis Terrarum. 1572-1618

(Faksimile-Ausgabe Bd.1-3).

Kassel: Bärenreiter.

Bridle, James (2018). New Dark Age.

Technology, Knowledge and the End of the Future. London: Verso. 
Bianchi, Paolo/Folie, Sabine/Offenes Kulturhaus Linz (Hg.) (1997): Atlas Mapping. Künstler als Kartographen, Kartographie als Kultur (Magazin 4). Katalog zur Ausstellung vom 6. Juni bis 11. Juli 1997 im Offenen Kulturhaus Linz und vom 28. Februar bis 23. April 1998 im Kunsthaus Bregenz. Wien: Turia + Kant.

Brotton, Jerry (2014). Die Geschichte der Welt in zwölf Karten.

München: Bertelsmann.

Brus, Jan/Vondrakova, Alena/Vozenilek, Vit. (Hg.) (2015). Modern Trends in Cartography. Cham: Springer.

Burckhardt, Lucius/Blumenthal, Silvan/Schmitz, Martin (2012). Design ist unsichtbar. Entwurf, Gesellschaft \& Pädagogik. Berlin: Martin Schmitz.

Burckhardt, Lucius/Fezer, Jesko/Schmitz, Martin (2004). Wer plant die Planung? Architektur, Politik und Mensch.

Kassel: Martin Schmitz.

Bureau d'Études (2013). An Atlas of Agendas. Mapping the Power, Mapping the Commons. Eindhoven: Onomatopee.

Buschauer, Regine \& Willis, Katharine S. (2013). Locative Media. Medialität und Räumlichkeit. Multidisziplinäre Perspektiven zur Verortung der Medien. Bielefeld: transcript.

Carr, Nicholas (2010). Wer bin ich, wenn ich online bin ... und was macht mein Gehirn solange? Wie das Internet unser Denken verändert. München: Blessing.

Carr, Nicholas (2014). Abgehängt. Wo bleibt der Mensch, wenn Computer entscheiden? München: Hanser.

Castells, Manuel (2001). Der Aufstieg der Netzwerkgesellschaft. Band 1: Das Informationszeitalter. Wirtschaft. Gesellschaft. Kultur. Wiesbaden: VS Verlag für Sozialwissenschaften.

Chapman, Kate (2015). «Commoning in Katastrophenzeiten. Das OpenStreetMapTeam für humanitäre Einsätze.» In: Helfrich, Silke/Bollier,

David/Heinrich-Böll-Stiftung (Hg.):

Die Welt der Commons. Muster gemeinsamen Handelns.

Bielefeld: transcript, S. 200 - 203.
Chatwin, Bruce (1990). Traumpfade. München/Wien: Carl Hanser.

Chen, Jessie Y.C. \& Fragomeni, Gino (2018). Virtual, Augmented and Mixed Reality: Applications in Health, Cultural Heritage, and Industry (Lecture Notes in Computer Science 10910). 10th International Conference, VAMR 2018, Held as Part of $\mathrm{HCl}$ International 2018, Las Vegas vom 15. - 20. Juli 2018, Proceedings Teil II. Cham: Springer.

Colomina, Beatriz \& Wigley, Mark (2016). Are We Human? Notes on an Archaeology of Design. Zürich: Lars Müller.

Crampton, Jeremy W. (2010). Mapping. A Critical Introduction to Cartography and GIS (Critical Introductions to Geography). Malden, Mass: Wiley-Blackwell.

De Certeau, Michel (1988). Kunst des Handelns [fr. Ausgabe 1980]. Berlin: Merve.

Debord, Guy (1996). Die Gesellschaft des Spektakels. Hg. v. Klaus Bittermann. Berlin: Edition Tiamat.

Deleuze, Gilles, \& Guattari, Félix (2005).

Tausend Plateaus. Kapitalismus und

Schizophrenie (6. Auflage). Berlin: Merve.

Deuleuze, Gilles (1993). «Postskriptum über die Kontrollgesellschaften.» In: Deleuze, Gilles: Unterhandlungen 1972 - 1990,

Frankfurt am Main: Suhrkamp, S. 254-262.

Denil, Mark (2011). «The Search for a Radical Cartography.» Cartographic Perspectives 68, S.7-28.

Denzinger, Jochen (2018). Das Design digitaler Produkte. Entwicklungen, Anwendungen, Perspektiven.

Basel: Birkhäuser.

Peluso, Nancy Lee (2011). «Whose Woods are These? Counter-Mapping Forest Territories in Kalimantan, Indonesia.» In: Dodge, Martin/Kitchin, Rob/Perkins, Chris (Hg.): The Map Reader.

Theories of Mapping Practice and

Cartographic Representation.

Chichester: Wiley-Blackwell, S. 422 - 429.

Dodge, Martin (2016). Mapping. Critical Concepts in Geography (Critical concepts in Geography). London: Routledge, Taylor \& Francis.

Chatwin, Bruce (1987). Songlines.

London: Vintage. 
Döring, Jörg \& Thielmann, Tristan (Hg.) (2009a). Mediengeographie. TheorieAnalyse-Diskussion.

Bielefeld: transcript.

Döring, Jörg \& Thielmann, Tristan

(Hg.) (2009b). Spatial Turn. Das

Raumparadigma in den Kultur- und

Sozialwissenschaften (2., unveränderte

Auflage). Bielefeld: transcript.

Döring, Jörg (2010). "Spatial Turn.»

In: Günzel, Stephan (Hg.): Raum.

Ein interdisziplinäres Handbuch.

Stuttgart: J.B. Metzler, S.90-99.

Drucker, Johanna (2014). Graphesis:

Visual Form of Knowledge Production.

Cambridge: Harvard University Press.

Dunne, Anthony \& Raby, Fiona (2014).

Speculative Everything. Design, Fiction, and Social Dreaming.

Cambridge: MIT Press.

Dünne, Jörg \& Günzel, Stephan (2006).

Raumtheorie. Grundlagentexte aus

Philosophie und Kulturwissenschaften.

Frankfurt am Main: Suhrkamp.

Eckert, Max (1921). Die

Kartenwissenschaft. Forschungen und

Grundlagen zu einer Kartographie als

Wissenschaft. Berlin/Leipzig: Vereinigung

Wissenschaftlicher Verleger.

Eco, Umberto (1991). Entwurf einer

Theorie der Zeichen.

München: Wilhelm Fink.

Eco, Umberto (2002). Einführung in die Semiotik. München: Wilhelm Fink.

Ehrenfels, Christian (1978): «Über

Gestaltqualitäten.» In: Weinhandl,

Ferdinand (Hg.): Gestalthaftes

Sehen. Ergebnisse und Aufgaben der

Morphologie. Zum Hundertjährigen

Geburtstag von Christian von Ehrenfels.

Nachdruck der Ausgabe von 1937.

Darmstadt: Wissenschaftliche

Buchgesellschaft, S.61-63.

Eibl, Ralf (2002). «Wege aus dem Chaos.» brandeins Magazin 04, o.S.

www.brandeins.de/magazine/brand-

eins-wirtschaftsmagazin/2002/

selbststaendigkeit/wege-aus-dem-chaos

(abgerufen am 18.05.2019).

Elsberg, Marc (2014). Zero. Sie wissen, was du tust. München: Blanvalet.
Erlhoff, Michael \& Marshall, Tim (2008).

Wörterbuch Design: Begriffliche

Perspektiven des Design (Board of

international research in design, BIRD).

Basel: Birkhäuser.

Farman, Jason (2010). «Mapping the

Digital Empire: Google Earth and

the Process of Postmodern Cartography.»

New Media \& Society 12(6), S. $869-888$.

www.doi.org/10.1177/1461444809350900

(abgerufen am 25.06.2019).

Farman, Jason (2012). Mobile interface theory. Embodied space and locative media. New York: Routledge.

Fassler, Manfred (2009). «Cybernetic Localism: Space, Reloaded.» In: Döring, Jörg \& Thielmann, Tristan (Hg.):

Spatial Turn. Das Raumparadigma in den Kultur- und Sozialwissenschaften. Bielefeld: transcript, S. $185-217$.

Felgenhauer, Tilo \& Gäbler, Karsten (2018). Geographies of Digital

Culture (Routledge Research in Culture, Space and Identity). London: Routledge.

Fendly, Tim (2014). «What Guides us Should be Reliable.» In: Luca School of Arts (Hg.):

Thoughts on Designing Information.

Zürich: Lars Müller Publishers, S. 62 - 67.

Florida, Richard L. (2002). The Rise of the Creative Class. New York: Basic Books.

Florida, Richard L. (2005). Cities and the Creative Class. New York: Routledge.

Flügge, Matthias/Kudielka, Robert/

Lammert, Angela (2007). Raum. Orte der

Kunst, Nürnberg: Verlag für Moderne Kunst.

Foer, Franklin (2018). Welt ohne Geist.

Wie das Silicon Valley freies Denken und

Selbstbestimmung bedroht.

München: Blessing.

Foucault, Michel (2006). «Von anderen

Räumen.» In: Dünne, Jörg \& Günzel, Stephan

(Hg.): Raumtheorie. Grundlagentexte aus

Philosophie und Kulturwissenschaften.

Frankfurt am Main: Suhrkamp, S.317-329.

Franke, Anselm/Weizman, Eyal/Sheikh,

Shela, Goldsmiths, \& Haus der Kulturen

der Welt Berlin (2014). Forensis.

The Architecture of Public Truth.

Berlin: Sternberg Press.

Galloway, Alexander R. (2012).

The Interface Effect. Cambridge: Polity. 
Garrett, Jesse J. (2000). «The Elements of Visual User Interface.» www.jjg.net/elements/pdf/elements.pdf (abgerufen am 11.09.2017).

Graham, Mark \& Zook, Matthew (2013). «Augmented Realities and Uneven Geographies: Exploring the Geolinguistic Contours of the Web.» Environment and Planning A: Economy and Space 45(1), S. 77 - 99. www.doi.org/10.1068/a44674 (abgerufen am 03.04.2019).

Graham, Stephen D. N. (2005). «Softwaresorted Geographies.» Progress in Human Geography 29(5), S. $562-580$. www. doi.org/10.1191/0309132505ph568oa (abgerufen am 21.05.2019).

Greenfield, Adam (2018). Radical Technologies. The Design of Everyday Life (Paperback ed.). London: Verso.

Günzel, Stephan (2009). Raumwissenschaften. Frankfurt am Main: Suhrkamp.

Günzel, Stephan (2010). Raum. Ein interdisziplinäres Handbuch. Stuttgart: J.B. Metzler.

Günzel, Stephan (2017). Raum. Eine kulturwissenschaftliche Einführung (Edition Kulturwissenschaft 143). Bielefeld: transcript.

Hahn, Hans Peter (2014). Materielle Kultur. Eine Einführung (Ethnologische Paperbacks) (2., überarbeitete Auflage). Berlin: Reimer.

Hall, Peter (2008). «Fligth Paths.» In: Abrams, Janet \& Ders.: Else/where: Mapping New Cartographies of Networks and Territories. Minneapolis: University of Minnesota, S. 280-289.

Haraway, Donna J. (2018). Unruhig bleiben. Die Verwandtschaft der Arten im Chthuluzän. Frankfurt am Main: Campus.

Harley, J. B. (2001). The New Nature of Maps. Essays in the History of Cartography. Baltimore, Md: Johns Hopkins University Press.

Harley, J. B. (1989). «Deconstructing the map.» Cartographia 26(2), S.1-20.

Harmon, Katharine (2004). You are here. Personal geographies and other maps of the imagination. New York: Princeton Architectural Press.
Harmon, Katharine (2009). The Map as Art. Contemporary Artists Explore Cartography. New York: Princeton Architectural Press.

Hartmann, Frank \& Bauer, Erwin K. (Hg.) (2006). Bildersprache. Otto Neurath, Visualisierungen. Wien: WUV.

Harvey, David (1990). The Condition of Postmodernity. An Enquiry into the Origins of Cultural Change. Oxford, UK/Malden, MA: Blackwell.

Helfrich, Silke \& Bollier, David (Hg.)

(2015). Die Welt der Commons.

Muster gemeinsamen Handelns.

Bielefeld: transcript.

Hellige, Hans Dieter (2008). Mensch-

Computer-Interface. Zur Geschichte und

Zukunft der Computerbedienung.

Bielefeld: transcript.

Hemment, Drew (2006). "Locative Arts.» Leonardo 39(4), S. 348-355.

eprints.lancs.ac.uk/30971/1/leon-

1.2006.39.4.348.pdf (abgerufen am 05.12.2013).

Hemmersam, Peter/Aspen, Jonny/ Morrison, Andrew/Sem, Idunn/Havnør, Martin (2015). «Exploring Locative Media for Cultural Mapping.» In: De Souza e Silva, Adriana/Sheller, Mimi: Mobility and Locative Media. Mobile Communication in Hybrid Spaces. London: Routledge, S. $167-187$.

Hight, Jeremy (2013). «Locative Narrative.» In: Buschauer, Regine \& Willis, Katharine S. (Hg.): Locative Media. Medialität und Räumlichkeit. Multidisziplinäre Perspektiven zur Verortung der Medien. Bielefeld: transcript, S. 243-251.

Hilger, Christina (2011). Vernetzte Räume. Plädoyer Für Den Spatial Turn in der Architektur (Materialitäten 15).

Bielefeld: transcript.

Holmes, Brian (2007). «Network Maps, Energy Diagrams: Structure and Agency in the Global System.» In: Ders.: Escape the Overcode. Activist Art in the Control Society.

www.brianholmes.wordpress.com/2009/ 01/19/book-materials/

(abgerufen am 12.09.2017).

Hookway, Branden (2014). Interface. Cambridge, Mass: MIT Press. 
Howard, Pamela. (2009). What is scenography? (Theatre concepts) (2. Auflage). London: Routledge.

loannides, Marinos (Hg.). (2018). Digital Cultural Heritage. Final Conference of the Marie Skłodowska-Curie Initial Training Network for Digital Cultural Heritage, Olimje, Slowenien, 23. - 25. Mai 2017, revised selected papers. Cham: Springer.

Jacobs, Jane (1961). The Death and Life of Great American Cities.

New York: Random House.

Johnson, Steven (1999). Interface Culture. Wie neue Technologien Kreativität und Kommunikation verändern.

Stuttgart: Klett-Cotta.

Junge, Barbara (2012). The Digital Turn. Design in the Era of Interactive Technologies. Berlin: eLab Weissensee Academy of Art.

Kandinsky, Wassily (1955). Punkt und Linie zu Fläche. Beitrag zur Analyse der malerischen Elemente.

Bern-Bümpliz: Benteli-Verlag.

Kant, Immanuel (1977). Was heißt: sich im Denken orientieren? Werke in zwölf Bänden (Bd. 5). Frankfurt am Main:

Suhrkamp. Erstdruck in: Berlinische Monatsschrift, Oktober 1786, S. 304 - 330. www.zeno.org/nid/20009189815 (abgerufen am 10.04.2018).

Kepes, Gyorgy (1944). Language of Vision. Chicago: Theobald.

Kitchin, Rob \& Dodge, Martin (2011). Code/space. Software and Everyday Life. Cambridge, Mass.: MIT Press.

Klee, Paul (1979). Beiträge zur bildnerischen Formenlehre. Faksimilierte Ausgabe des Originalmanuskripts von erstem Vortragszyklus am staatlichen Bauhaus Weimar 1921/22. Hg. v. Jürgen Glaesemer. Basel: Schwabe.

Kollektiv Orangotango (2018). This is Not an Atlas. A Global Collection of Counter Cartographies (Sozial- und Kulturgeographie 26). Bielefeld: transcript.

Krippendorff, Klaus (2012). Die semantische Wende. Eine neue Grundlage für Design (Schriften zur Gestaltung).

Basel: Birkhäuser.
Krygier, John, \& Wood, Denis (2005).

Making Maps. A Visual Guide to Map Design for GIS (2. Auflage).

New York: Guilford Press.

Kurgan, Laura (2013). Close Up at a Distance. Mapping, Technology, and Politics. New York: Zone Books.

Lammert, Angela (2005). Topos Raum.

Die Aktualität des Raumes in den

Künsten der Gegenwart.

Nürnberg: Verlag für Moderne Kunst.

Lanier, Jaron (2014). Wem gehört die Zukunft? Du bist nicht der Kunde

der Internet-Konzerne, du bist ihr Produkt. Hamburg: Hoffmann und Campe.

Laurel, Brenda (1990). The Art of Humancomputer Interface Design. Reading:

Addison-Wesley.

Le Monde diplomatique (Hg.) (2009).

Atlas der Globalisierung.

Lahr: Druckhaus Kaufmann.

Lefebvre, Henri (2006). «Die Produktion des Raums.» [fr. Ausgabe 1974] In: Dünne, Jörg \& Günzel, Stephan (Hg.): Raumtheorie. Grundlagentexte aus Philosophie und Kulturwissenschaften. Frankfurt am Main: Suhrkamp, S. $330-342$.

Lehnert, Gertrud (Hg.) (2011). Raum und Gefühl. Der Spatial Turn und die neue Emotionsforschung. Bielefeld: transcript.

Leszczynski, Agnieszka (2019).

«Spatialites.» In: Ash, James/Kitchin, Rob/Leszczynski, Agnieszka (Hg.):

Digital Geographies.

London: Sage Publications, S.13-23.

Lynch, Kevin (1960): The Image of the City. Cambridge: MIT Press.

Lynch, Kevin (1996). «Reconsidering The Image of the City.» In: Banerjee, Tridib \& Southworth, Michael. City Sense and City Design. Cambridge, Mass: MIT Press, 247 - 256. Lynch, Kevin (2001). Das Bild der Stadt [engl. Ausgabe 1960].

Basel: Birkhäuser.

MacEachren, Alan M. (1995). How Maps

Work. Representation, Visualization, and Design. New York: Guilford Press. 
Manovich, Lev (2005). «Poetik des erweiterten Raums.» In: Akademie der Künste (Hg.): Topos Raum. Die Aktualität des Raumes in den Künsten der Gegenwart. Nürnberg: Verlag für Moderne Kunst, S. $337-349$.

Manovich, Lev (2009). «Geomedien: Raum als neue Medien-Plattform? Ein Interview mit Lev Manovich.» In: Döring, Jörg \& Thielmlann, Tristan (Hg.): Mediengeographie. Theorie - Analyse Diskussion.

Bielefeld: transcript, S. 383-396.

Mareis, Claudia (2011). Design als Wissenskultur. Interferenzen zwischen Design- und Wissensdiskursen seit 1960 (Studien zur visuellen Kultur 16). Bielefeld: transcript.

Marshall, Tim (2017). Die Macht der Geographie. Wie sich Weltpolitik anhand von 10 Karten erklären lässt

(aktualisierte und erweiterte Neuausgabe) München: dtv.

Mattern, Shannon (2015). Deep Mapping the Media City. Minnesota: University of Minnesota Press.

Mattern, Shannon (2017). Mapping's Intelligent Agents. Places Journal, September.

www.doi.org/10.22269/170926

(abgerufen am 09.11.2017).

McLuhan, Marshall \& Powers, Bruce R. (1989). The Global Village. Transformations in World Life and Media in the 21st Century (Communication and Society). New York: Oxford University press.

Meier, Patrick (2015). «The Rise of Digital Humanitarians.» In: Ders.: Digital Humanitarians. How Big Data is Changing the Face of Humanitarian Response. Boca Raton: CRC Press, S.1-22.

Mersch, Dieter (2011). «Fraktale Räume und multiple Aktionen. Überlegungen zur Orientierung in komplexen medialen Umgebungen.» In: Lehnert, Gertrud: Raum und Gefühl. Der Spatial Turn und die neue Emotionsforschung.

Bielefeld: transcript, S. 49-62.

Metahaven (2008). White Night. Before A Manifesto. Eindhove: Onomatopee.
Milgram, Paul \& Kishino, Fumio (1994).

A Taxonomy of Mixed Reality Visual Displays. IEICE Transactions on Information and Systems E77-D 12(12),

S. $1321-1329$.

Monmonier, Mark S. (2018). How to

Lie With Maps (3. Auflage).

Chicago: The University of Chicago Press.

Narzt, Wolfgang/Pomberger, Gustav/

Ferscha, Alois/Kolb, Dieter/Müller, Reiner/

Wieghardt, Jan/Hörtner, Horst/Lindinger,

Christoph (2004). «A New Visualization

Concept for Navigation Systems.» In:

Stary, Christian \& Stephanidis, Constantine

(Hg.): User-Centered Interaction Paradigms

for Universal Access in the Information

Society. 8. ERCIM Workshop on User Interfaces for All, Wien vom 28. - 29. Juni 2004, Revised Selected Papers.

Berlin/Heidelberg: Springer, S. $440-451$.

Narzt, Wolfgang/Pomberger, Gustav/ Ferscha, Alois/Kolb, Dieter/Müller, Reiner/ Wieghardt, Jan/Hörtner, Horst/Lindinger, Christoph (2006). Augmented Reality Navigation Systems. Universal Access in the Information Society 4(3), S.177-187.

Norman, Donald A. (2018). «Die Zukunft des Design: Die Entscheidung für einen Weg? Nimm beide.» In: Denzinger, Jochen (Hg.): Das Design digitaler Produkte. Entwicklungen, Anwendungen, Perspektiven. Basel: Birkhäuser, S.16-23.

O’Keefe, John \& Nadel, Lynn (1978).

The Hippocampus as a Cognitive Map. Oxford: Clarendon Press.

O'Rourke, Karen (2013). Walking and Mapping. Cambridge, Mass.: MIT Press.

Obrist, Hans-Ulrich (2014). Mapping it Out. An Alternative Atlas of Contemporary Cartographies. London: Thames \& Hudson.

Offenhuber, Dietmar \& Ratti, Carlo (2012). «Reading the City. Reconsidering Kevin Lynch's Notion of Legiblility in the Ditgital Age.» In: Junge, Barbara/Berzina, Zane/Scheiffele, Walter/Westerveld, Wim/Zwick, Carola (Hg.): The Digital Turn. Design in the Era of Interactive Technology. Berlin: Park Books, S. $215-224$.

Offenhuber, Dietmar \& Ratti, Carlo (2013).

Die Stadt entschlüsseln. Wie Echtzeitdaten den Urbanismus verändern (Bauwelt Fundamente 150). Basel: Birkhäuser. 
Panofsky, Erwin (1980). «Die Perspektive als symbolische Form.» In: Ders.: Aufsätze zu Grundfragen der Kunstwissenschaft. Hg. v. Hariolf Oberer \& Egon Verheyen. Berlin: Volker Spiess, S.99-167.

Papanek, Victor, \& Pumhösl, Florian (2009). Design für die reale Welt.

Anleitungen für eine humane Ökologie und sozialen Wandel (Edition Angewandte).

Wien: Springer.

Pater, Ruben (2016). The Politics of Design. A (Not so) Global Manual for Visual Communication. Amsterdam: BIS.

Pearson, Mike \& Shanks, Michael (2001). Theatre, Archaeology.

London: Routledge.

Peirce, Charles S. (1983). Phänomen und Logik der Zeichen.

Frankfurt am Main: Suhrkamp.

Perec, Georges (2013). Träume von

Räumen. Zürich: Diaphanes.

Pfeiffer, Max/Dünte, Tim/Schneegass,

Stephan/Alt, Florian/Rohs, Michael (2015).

Cruise Control for Pedestrians. Controlling

Walking Direction using Electrical Muscle

Stimulation. www.hci.uni-hannover.de/

papers/pfeiffer2015CHICruise.pdf

(abgerufen am 01.03.2016).

Polanyi, Michael (1985). Implizites Wissen. Frankfurt am Main: Suhrkamp.

Popplow, Laura \& Scherffig, Lasse (2013). «Locative Arts - neue Erzählung des Raums?"In: Buschauer, Regine \& Willis, Katharine S. (Hg.): Locative Media. Medialität und Räumlichkeit. Multidisziplinäre Perspektiven zur Verortung der Medien.

Bielefeld: transcript, S. 277-295.

Proust, Marcel (1994-2003). Auf der Suche nach der verlorenen Zeit (7 Bde.). Frankfurt am Main: Suhrkamp.

Proust, Marcel (2001). In Swanns Welt (Bd.1). Frankfurt am Main: Suhrkamp Verlag.

Ramthun, Roland (2012). «Offene Geodaten durch OpenStreetMap.» In: Herb, Ulrich (Hg.): Open Initiatives. Offenheit in der digitalen Welt und Wissenschaft. Saarbrücken: Universaar, S. $159-184$.
Reckwitz, Andreas (2014). Die

Erfindung der Kreativität. Zum Prozess gesellschaftlicher Ästhetisierung (4. Auflage). Berlin: Suhrkamp.

Reckwitz, Andreas (2017). Die Gesellschaft der Singularitäten. Zum Strukturwandel der Moderne (3. Auflage).

Berlin: Suhrkamp.

Richterich, Annika \& Schabacher, Gabriele (2011). Raum als Interface. Siegen: universi - Universitätsverlag Siegen.

Robinson, Arthur H. (2010). The Look of Maps. New York: Esri Press.

Roesler-Keilholz, Silke (2013). «Maps That Watch. Zur immersiven Kartographie am Beispiel von Google.» In: Buschauer, Regine \& Willis, Katharine S. (Hg.): Locative Media. Medialität und Räumlichkeit. Multidisziplinäre Perspektiven zur Verortung der Medien. Bielefeld: transcript, S.167-182.

Russegger, Georg/Tarasiewicz, Matthias/Wlodkowski, Michal (Hg.) (2011) Coded cultures. New Creative Practices out of Diversity. Wien: Springer.

Samida, Stefanie (2014). Handbuch Materielle Kultur. Bedeutungen, Konzepte, Disziplinen. Stuttgart: Metzler.

Schlögel, Karl (2011). Im Raume lesen wir die Zeit. Über Zivilisationsgeschichte und Geopolitik. Frankfurt am Main: Fischer Taschenbuchverlag.

Schramm, Manuel (2012). «Kartenwissen und digitale Kartographie. Technischer Wandel und Transformation des Wissens im 20. Jahrhundert.» In: Günzel, Stephan \& Nowak, Lars (Hg.): KartenWissen.

Territoriale Räume zwischen Bild und Diagramm. Wiesbaden: Reichert, S. $451-460$.

Schramm, Manuel (2013). «Digitale Kartographie und Locative Media. Eine historische Perspektive.» In: Buschauer, Regine \& Willis, Katharine S. (Hg.):

Locative Media. Medialität und Räumlichkeit. Multidisziplinäre Perspektiven zur Verortung der Medien. Bielefeld: transcript, S.123-135.

Schranz, Christine (2013). Von der Dampfzur Nebelmaschine. Szenografische Strategien zur Vergegenwärtigung von Industriegeschichte am Beispiel der Ruhrtriennale (Szenografie \& Szenologie). Bielefeld: transcript. 
Schranz, Christine (2014). «Augmented Reality in Design. Thinking about Hybrid Forms of Virtual and Physical Space in Design. „ In: Marcus, Aaron (Hg.). Design, User Experience, and Usability: User Experience Design for Diverse Interaction Platforms and Environments. Third International Conference, DUXU 2014, Kreta, 22. - 27. Juni 2014, Teil II, LNCS 8518. Heidelberg: Springer, S. $624-635$.

Schranz, Christine (2017). «Die Karte als Interface.» In: Institut für Immersive Medien (Hg.): Interaktive Medien: Interfaces - Netze - Virtuelle Welten (Jahrbuch Immersive Medien 2016). Marburg: Schüren, S. 27-37.

Schranz, Christine (2018). «Commons for Mapping: How Social Computing Changes the Design of Interfaces.» Interface Critique Journal 1. Hg. v. Florian Hadler, Alice Soiné und Daniel Irrgang, S.168-175.

Schwanke-Seer, Michael (2013). «Digitale Informationsübermittlung.» In: Kling, Beate \& Krüger, Torsten: Orientierung im Raum (Edition Detail). München: Detail, S. $136-143$.

Sennett, Richard (2008). Handwerk. Berlin: Berlin.

Mattern, Shannon (2017). «Mapping's Intelligent Agents. » Places Journal, September. www.doi.org/10.22269/170926 (abgerufen am 17.10.2017).

Sommer, Bernd, \& Welzer, Harald (2014). Transformationsdesign. Wege in eine zukunftsfähige Moderne.

München: Oekom.

Sommerer, Christa/Mignonneau, Laurent/ King, Dorothée (2008). Interface Cultures. Articsitc Aspects of Interaction.

Bielefeld: transcript.

Stalder, Felix (2016). Kultur der Digitalität. Berlin: Suhrkamp.

Stankowski, Anton (1967). Funktion und ihre Darstellung in der Werbegrafik. Die Sichtbarmachung unsichtbarer Vorgänge. Teufen: Niggli.

Starck, Philippe (2018). «Ich war immer ein bisschen autistisch.» Zeitmagazin 15, o.S. www.zeit.de/zeit-magazin/2018/15/ philippe-starck-produktdesign-interview/ komplettansicht

(abgerufen am 21.05.2019).
Steyerl, Hito (2015). «Duty-Free Art.»

E-flux Journal 63, S.1-17.

worker01.e-flux.com/pdf/article

8996937.pdf (abgerufen am 18.10.2018).

Steyerl, Hito/Cruz Yábar/Almudena,

Rodríguez, Mafalda, \& Museo Nacional

Centro de Arte Reina Sofía (2015).

Hito Steyerl. Duty-free Art. Madrid:

Museo Nacional Centro de Arte Reina Sofía.

Stirnemann, Julia Mia (2018). Über

Projektionen: Weltkarten und Weltanschau-

ungen. Von der Rekonstruktion zur

Dekonstruktion, von der Konvention

zur Alternative (Image 147).

Bielefeld: transcript.

Thompson, Nato (Hg.) (2008). Experimental

Geography. Radical Approaches to

Landscape, Cartography, and Urbanism.

New York: Melville.

Tkacz, Nathaniel (2015). Wikipedia and

the Politics of Openness. Chicago:

The University of Chicago Press.

Tolman, Edward (1948). «Cognitive Maps

in Rats and Men.» Psychological Review 55, S. $189-208$

www.psychclassics.yorku.ca/Tolman/

Maps/maps.htm (abgerufen am 21.05.2019)

Tufte, Edward R. (1997). Visual Explanations. Images and Quantities, Evidence and

Narrative.

Cheshire: Graphics Press.

Tufte, Edward R. (2001). Envisioning Information (8. Auflage). Cheshire, Connecticut: Graphics Press.

Tufte, Edward R. (2004). The Visual Display of Quantitative Information. Cheshire: Graphics Press.

Vaidhyanathan, Siva (2011). The Googlization of Everything (And Why We Should Worry).

Berkeley: University of California Press.

Verwaltung der Öffentlichen

Materiallagerplätze Basel-Dreispitz (1951).

50 Jahre Dreispitz. Kleine Gedenkschrift

zum Anlass des 50-jährigen Bestehens

der Öffentlichen Material-Lagerplätze

Basel-Dreispitz und der kantonalen

Industriegeleise Basel-St. Johann.

Basel: Verwaltung der Öffentlichen

Materiallagerplätze Basel-Dreispitz. 
Virilio, Paul (2006). «Die Auflösung des

Stadtbilds.» In: Dünne, Jörg/

Günzel, Stephan (Hg.): Raumtheorie.

Grundlagentexte aus Philosophie und

Kulturwissenschaften. Frankfurt am Main:

Suhrkamp, S. 261- 273.

Wanner, Gustaf Adolf (1972). 50 Jahre Basler Zollfreilager 1922 -1972. 0. O.

Weiser, Mark (1991). The Computer for the 21st Century. www.Iri.fr/ mbl/ Stanford/CS477/papers/Weiser-SciAm.pdf (abgerufen am 06.09.2017).

Welsch, Wolfgang (1990). «Perspektiven für das Design der Zukunft.» In:

Ders.: Ästhetisches Denken. Stuttgart, S. $201-218$.

Werlen, Benno (2008). «Körper, Raum und medial Repräsentation.» In: Döring, Jörg\&Thielmann, Tristan (Hg.):

Spatial Turn. Das Raumparadigma in den Kultur- und Sozialwissenschaften. Bielefeld: transcript, S. $365-392$.

Woletz, Julie (2016). Human-Computer Interaction. Kulturanthropologische Perspektiven auf Interfaces.

Darmstadt: Büchner-Verlag.

Wood, Denis (1992). The Power of Maps (Mappings). New York: Guilford Press.

Wood, Denis (2003). «Cartography is Dead (Thank God!).» Cartographic Perspectives (45), S. 4-7. www.doi.org/10.14714/CP45.497 (abgerufen am 07.07.2019)

Wood, Denis/Fels, John/Krygier, John (2010). Rethinking the Power of Maps. New York: Guilford Press.

Zuboff, Shoshana (2018). Das Zeitalter des Überwachungskapitalismus.

Frankfurt am Main: Campus. 

Abb.1 C Courtesy of the Artist

Abb. 2 (C) 2019, ProLitteris, Zürich

Abb.3 Www.bureaudetudes.org (abgerufen am 08.04.2019), (C) Bureau d'Études

Abb. 4 www.vimeo.com/166807261 (abgerufen am 20.11.2018)

Abb. 6 C Courtesy of the Artist

Abb.7 Institut Experimentelle Design- und Medienkulturen (IXDM), (C) Christine Schranz

Abb. $8 \quad$ Wikimedia Commons, commons.wikimedia.org (abgerufen am 18.02.2016)

Abb. 9 Laura Kurgan, (C) Landsat, from EROS Data Center

Abb.10 Plant Ecology \& Conservation Lab, (C) Jon Evans

Abb.11 www.yelp.com (abgerufen am 22.10.2018)

Abb.12 (C) 2019 Axon Vibe AG, Luzern

Abb.13 C Fabio Soldati

Abb.14 (c) The Museum of London

Abb.15 Yuki OMORI

Abb.16 Farman 2010: 10, (C) Pacific Northwest National Laboratory's

Abb.17 C MIT SENSEable City Lab and Personal Robots Group of Media Lab

Abb.18 www.swisstopo.admin.ch (abgerufen am 26.06.2019), reproduziert mit Einwilligung von swisstopo (BA19056)

Abb.19 www.google.com/maps (03.04.2019), ( Google Maps 2019

Abb. $20 \quad$ Bertin 1967: 43

Abb. 21 Wikimedia Commons, commons.wikimedia.org (abgerufen am 08.05.2015)

Abb.22 Wikimedia Commons, commons.wikimedia.org (abgerufen am 13.05.2019)

Abb.23 Zentralbibliothek Zürich, Graphische Sammlung

Abb. 24 Zentralbibliothek Zürich, Kartensammlung

Abb.25 C Courtesy of the Artist, Foto: Google Earth

Abb.26 Courtesy of the Artist

Abb. $27 \quad$ www.waymo.com (abgerufen am 11.04.2018)

Abb. 28 www.hci.uni-hannover.de/papers/pfeiffer2015CHICruise.pdf (abgerufen am 24.10.2018)

Abb. 29 Zehn Hoch, (c) Eames Office, LLC

Abb.30 www.decolonialatlas.wordpress.com (abgerufen am 07.07.2019)

Abb.31 CArmin Weigel

Abb. 32 (C) Graham \& Zook 2013

Abb.33 www.openstreetmap.org (abgerufen am 31.05.2019)

Abb.34 www.hotosm.org (04.09.2017)

Abb.35 (C) The Buckminster Fuller Institute

Abb.36 www.theguardian.com/technology/2014/apr/22/google-mapsrussia-crimea-federation (abgerufen am 12.07.2018)

Abb.37 C Michael James Hermann und Margaret Wickens Pearce 
Abb.38 (C) The Museum of Modern Art, New York/Scala, Florence

Abb.39 realtime.waag.org (abgerufen am 08.05.2018)

Abb.40 Wikimedia Commons, commons.wikimedia.org (abgerufen am 12.09.2017)

Abb.41 Institut Experimentelle Design- und Medienkulturen (IXDM), (C) Christine Schranz

Abb.42 Courtesy Altman Siegel Gallery, San Francisco, (C) John Emerson und Trevor Paglen. www.strozzina.org/ ritrattidelpotere/e_paglen.php (abgerufen am 09.07.2019)

Abb.43 Tolman 1948: o.S.

Abb.44 Www.saulsteinbergfoundation.org (abgerufen am 09.05.2019), (C) 2019, ProLitteris, Zurich

Abb.45 Lynch 2001: 171.

Abb.46 www.decolonialatlas.wordpress.com/2016/04/12/inuitcartography/ (abgerufen am 08.05.2018)

Abb.47 C Applied Information Group

Abb.48 Google Maps, www.google.com (abgerufen am 16.05.2019)

Abb.49 Wikimedia Commons, commons.wikimedia.org (abgerufen am 09.05.2019)

Abb.50 Zentralbibliothek Zürich, Kartensammlung

Abb.51 Wikimedia Commons, commons.wikimedia.org (abgerufen am 09.05.2019), (C) Courtesy of the Artist

Abb.52 Wikimedia Commons, commons.wikimedia.org (abgerufen am 09.05.2019), (C) Courtesy of the Artist

Abb.53 Katrin Schoof

Abb.54 enterprise.foursquare.com/intersections/article/introducinghypertrending/ (abgerufen am 11.04.2019)

Abb.55 C Courtesy of the Artist

Abb.56 Tate Modern London, (c) Courtesy of the Artist

Abb.57 www.neme.org/texts/narrative-archaeology (abgerufen am 11.04.2019)

Abb.58 Christoph Merian Archiv

Abb.59 Hochschule für Gestaltung und Kunst Basel FHNW

Abb.60 www.louisvuitton.com (abgerufen am 05.12.2018)

Abb.61 www.artrank.com (abgerufen am 22.01.2019)

Abb. 62 Tom Bisig

Abb. 63 Fred Merz für die New York Times

Abb.64 (C) Atelier d'Architecture 3BM3

Abb. 65 Merle Ibach

Abb. 66 Merle Ibach

Abb.68 Samuel Hanselmann

Abb.69 Christoph Merian Archiv

Abb.70 Christoph Merian Archiv

Abb.73 Samuel Hanselmann

Abb. 87 Christoph Merian Archiv

Tab. 1 Abend 2013: 187 
Schranz, Christine (2018):

The Digital Memory of Palmyra. An Artistic Approach

Für einen internationalen Forschungsaufenthalt an der Winchester School of Art, University of Southampton (GB), initiierte Christine Schranz ein Forschungsprojekt mit dem Titel The Digital Memory of Palmyra. An Artistic Approach. Mittels des Mediums Kartografie erforschte sie zerstörte Kulturgüter im Nahen Osten, insbesondere in Syrien. Das Forschungsvorhaben bewegte sich im Spannungsfeld eines institutionalisierten Erinnerungsgedächtnisses (Top-down) sowie eines entstehenden digital-kulturellen Gedächtnisses (Bottom-up). Dabei wurden Fragen dazu aufgeworfen, inwieweit bisherige Top-downStrategien die einzig legitime Form zur Rekonstruktion und Erinnerung von Kultur(-Gütern) sind beziehungsweise ob sich im Zeitalter eines digitalen Gedächtnisses auch Bottom-up-Strategien bewähren und inwiefern sich diese von den herkömmlichen Strategien unterscheiden.

Schranz, Christine \& Wahbeh, Wissam (2017):

Photogrammetry-based 3-D Modelling for VR

In Zusammenarbeit mit dem Kooperationspartner Hochschule für Architektur, Bau und Geomatik FHNW Basel hat Christine Schranz ein Lehrformat zur Georeferenzierung des Untersuchungsareals erarbeitet. Zehn Studierende der Disziplin Geomatik haben innerhalb eines zweiwöchigen Workshops das Areal vermessen und die Rekonstruktion bestehender Gebäude und Objekte in 3-D-Modellen für den Einsatz in Virtual-Reality-Anwendungen erarbeitet. Die Daten werden in der Applikation (siehe: Anwendung) verwendet.

Schranz, Christine (2016):

Kosmos Dreispitz - Kultur digital erzählt

Mit sechs Studierenden aus unterschiedlichen Disziplinen hat sich Christine Schranz auf Spurensuche nach den verborgenen Erzählungen des Areals begeben. In künstlerisch-gestalterischer Praxis sind so fiktive wie reale Geschichten zum Kosmos Dreispitz entstanden, die neue (Ein-)Blicke in und auf das Areal mit seinen Nutzer_innen eröffnen. Die recherchierten Geschichten dienen als Inspiration für die Applikation (siehe: Anwendung).

Schranz, Christine (2016):

Dreispitz-Areal - eine alternative Kartographie

Mit acht Studierenden aus unterschiedlichen Disziplinen hat Christine Schranz den Campus der Künste erkundet.

Das Areal wurde zu Fuss und aus der Luft erforscht, physisch und digital vermessen und statisch-geologisch untersucht. Es wurden Farbproben entnommen, Lieblingsorte aufgespürt und das Schienennetz der ehemaligen Eisenbahn kartiert. In individueller gestalterisch-künstlerischer Praxis sind so acht alternative Kartografien entstanden. Die Resultate dienten als Auftakt für das Forschungsprojekt und wurden an der Hochschule für Gestaltung und Kunst FHNW Basel ausgestellt. 
Schranz, Christine \& Steudler, Sandro (2015):

Medialisierte Räume und verräumlichte Medien

Eine Vorlesungsreihe zum Thema Medialisierte Räume und verräumlichte Medien in Zusammenarbeit mit dem Künstler Sandro Steudler. Standen zur Jahrtausendwende die Konvergenz von älteren Medien im Digitalen zur Debatte, sollte mit dem Mobilwerden der Kommunikationstechnologien - bei ständigem Austausch und Teil des WWW - eine Medienanalyse vermehrt unter räumlichen Aspekten durchgeführt werden. Diese Perspektivenverschiebung ist umso virulenter, da sich mit dem anbrechenden Internet der Dinge und dem Vordringen von computergestützten Fertigungstechniken (z. B. 3-D-Printing) in den Alltag vermehrt Vermischungen von virtuellem mit physischem Raum als eine eigentliche Hybridisierung - als Virtual/Physical Reality stattfinden. 


\section{Danksagung}

Diese Publikation ist im Zusammenhang mit dem durch den Schweizer Nationalfonds geförderten Forschungsprojekt Augmented Space (2015 - 19) am Institut Experimentelle Design- und Medienkulturen der Hochschule für Gestaltung und Kunst FHNW in Basel entstanden. Ausgangspunkt dieser Schrift bildet meine forschungsbasierte Arbeit zu den Themen Raum, Karte, Design und Kommunikation in Lehre, Forschung und Praxis.

Mein Dank gilt Claudia Mareis vom Institut Experimentelle Design- und Medienkulturen sowie Andreas Wenger vom Institut Innenarchitektur und Szenografie, die mich von Anfang an bei diesem Projekt unterstützt und meine Forschungen ermöglicht haben.

Ein weiterer Dank geht an die Kolleginnen und Kollegen der HGK, die mich während des Prozesses begleitet und inspiriert haben. Namentlich sind dies Léonie Hässler, Shintaro Miyazaki und Jan Torpus sowie alle Studierenden, die an den Workshops und Seminaren im Zusammenhang mit diesem Forschungsprojekt teilgenommen haben.

Dem Institut Integrative Gestaltung, speziell Heinz Wagner, möchte ich für die finanzielle Unterstützung dieser Publikation danken.

Bedanken möchte ich mich auch bei dem Kooperationspartner Institut Geomatik IGEO, Hochschule für Architektur, Bau und Geomatik Muttenz FHNW, insbesondere bei Martin Christen, Stephan Nebiker und Wissam Wahbeh sowie bei der Abschlussklasse G2014 für das Erstellen des 3-D-Modells.

Ein weiterer Dank geht an Dimitri von Arx (BA Science in Geomatik), der mit seiner Abschlussarbeit einen Prototyp für eine VR-Applikation entwickelte und dessen Thesis Nutzungsmöglichkeiten von Geodaten mit Virtualund Augmented Reality auf dem Campus der Künste Dreispitz ich als Fachexpertin begleiten durfte.

Danken möchte ich auch der Christoph Merian Stiftung Basel, die mir ihr Archiv zum Zollfreilager Basel geöffnet hat. Dem Schweizerischen Nationalfonds danke ich für die grosszügige Förderung zur Durchführung dieses Forschungsprojekts.

Mein grösster Dank geht an das Projektteam: Urs Clement (IGEO) für die Programmierung der Applikation sowie die Beratung bei technischen Fragen zu in dieser Publikation behandelten Aspekten, Max Frischknecht und Philipp Möckli (Début Début, Basel) für das Interface Design und die Gestaltung dieser Publikation sowie Merle lbach (Institut Integrative Gestaltung | Masterstudio, FHNW) für die Projektassistenz. Mit viel Engagement, Herz und Elan haben sie zur erfolgreichen Durchführung der Forschung beigetragen. Tanja Jentsch danke ich für das umsichtige Lektorat.

Sandro Steudler möchte ich für das kritische Gegenlesen und die anregenden Gespräche danken. 

Lektorat: Tanja Jentsch, die Publikation folgt der Schweizer Rechtschreibung

Projektkoordination: Nora Kempkens

Herstellung: Bettina Chang, Amelie Solbrig

Layout, Covergestaltung und Satz:

Début Début, www.debutdebut.com

Papier: Magno Natural, $120 \mathrm{~g} / \mathrm{m}^{2}$

Lithographie: bildpunkt Druckvorstufen $\mathrm{GmbH}$, Berlin

Druck: Kösel GmbH\& Co. KG, Altusried

Publiziert mit Unterstützung des Schweizerischen

Nationalfonds zur Förderung der wissenschaftlichen

Forschung

\section{FN - NF}

SCHWEIZERISCHER NATIONALFONDS

ZUR FÖRDERUNG DER WISSENSCHAFTUCHEN FORSCHUNG

Library of Congress Control Number: 2019949997

Bibliografische Information der Deutschen Nationalbibliothek

Die Deutsche Nationalbibliothek verzeichnet

diese Publikation in der Deutschen Nationalbibliografie;

detaillierte bibliografische Daten sind im Internet

über www.dnb.de abrufbar.

DOI: www.doi.org/10.1515/978-3-0356-2025-2

\section{BY-NC-ND}

Dieses Werk ist lizenziert unter der Creative Commons Attribution-NonCommercial-NoDerivatives 4.0 Lizenz.

Weitere Informationen finden Sie unter WWW.creativecommons.org/licenses/by-nc-nd/4.0/.

Dieses Buch ist als Open-Access-Publikation verfügbar über www.degruyter.com.

Dieses Werk ist urheberrechtlich geschützt. Die dadurch begründeten Rechte, insbesondere die der Übersetzung, des Nachdrucks, des Vortrags, der Entnahme von Abbildungen und Tabellen, der Funksendung, der Mikroverfilmung oder der Vervielfältigung auf anderen Wegen und der Speicherung in Datenverarbeitungsanlagen, bleiben, auch bei nur auszugsweiser Verwertung, vorbehalten. Eine Vervielfältigung dieses Werks oder von Teilen dieses Werks ist auch im Einzelfall nur in den Grenzen der gesetzlichen Bestimmungen des Urheberrechtsgesetzes in der jeweils geltenden Fassung zulässig. Sie ist grundsätzlich vergütungspflichtig. Zuwiderhandlungen unterliegen den Strafbestimmungen des Urheberrechts.

ISBN 978-3-0356-2016-0

e-ISBN (PDF) 978-3-0356-2025-2

(C) 2020 Birkhäuser Verlag $\mathrm{GmbH}$, Basel

Postfach 44, 4009 Basel, Schweiz

Ein Unternehmen der Walter de Gruyter $\mathrm{GmbH}$, Berlin/Boston 